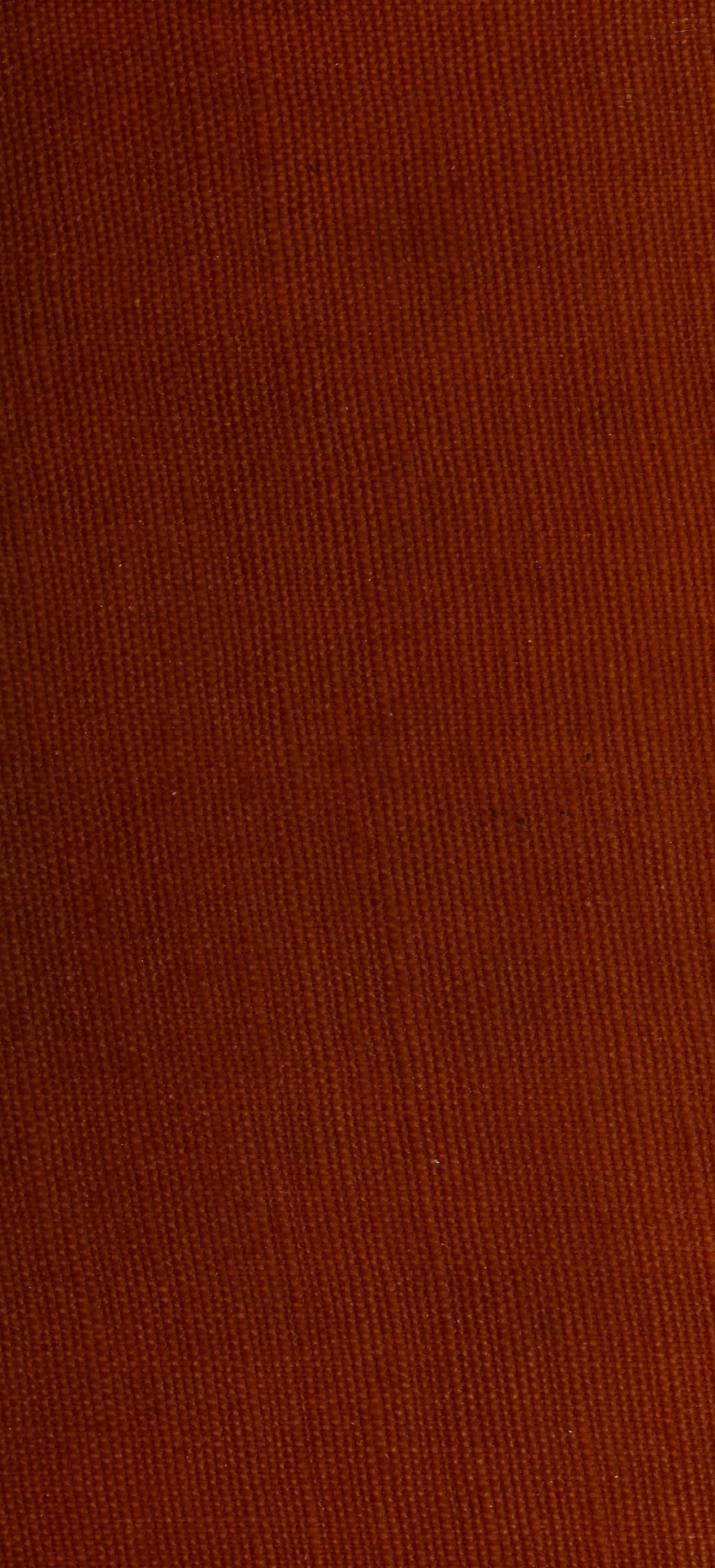




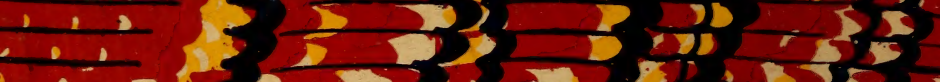

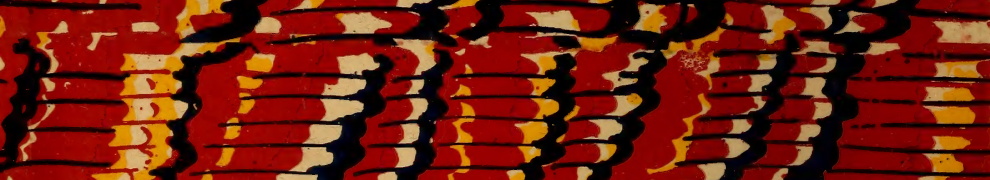

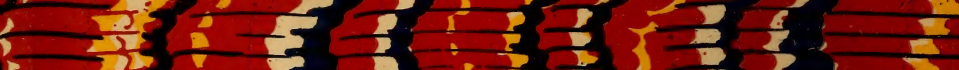

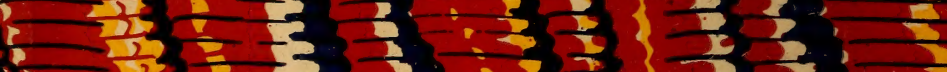

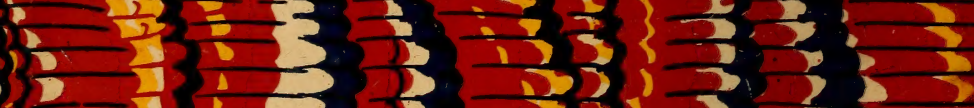

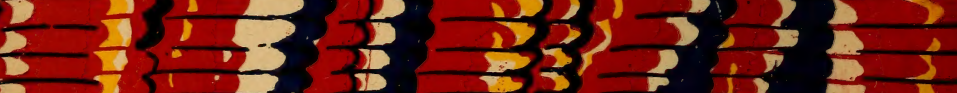

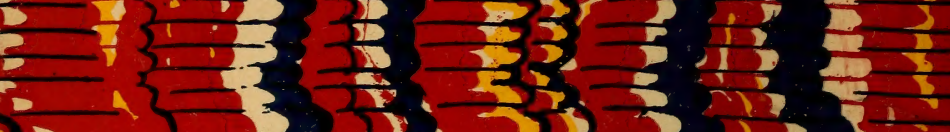

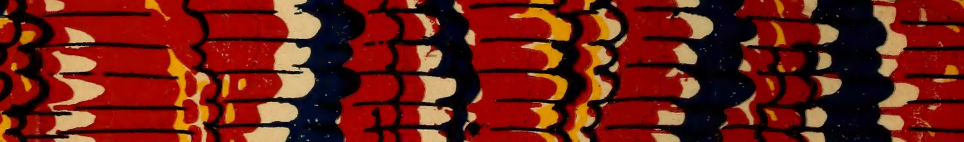

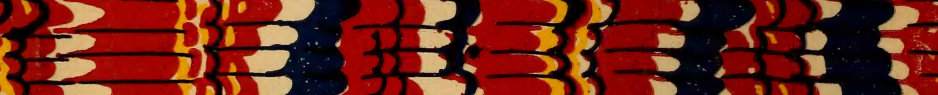

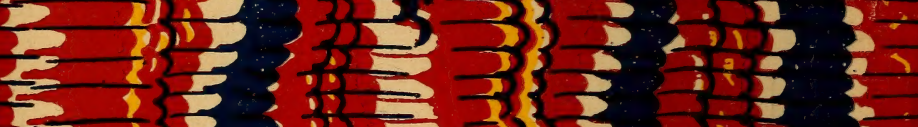

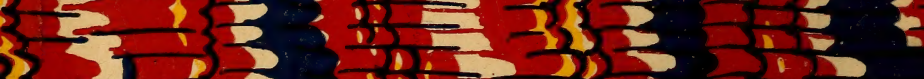

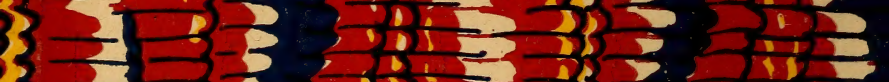

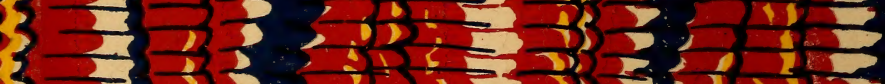

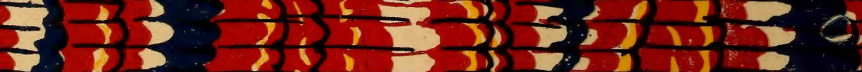

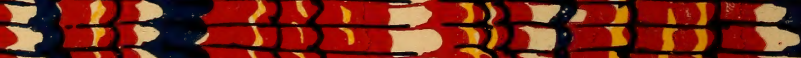

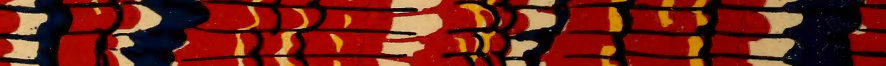

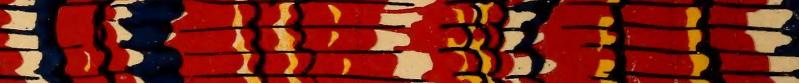

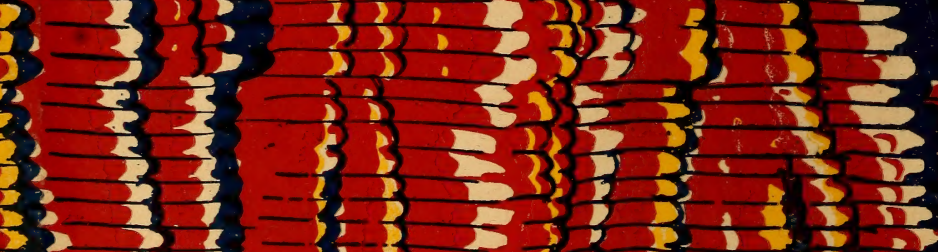

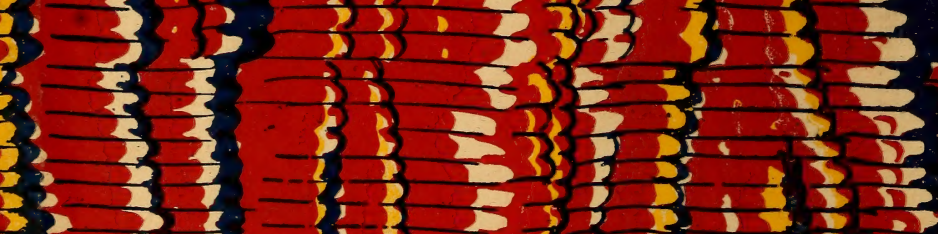

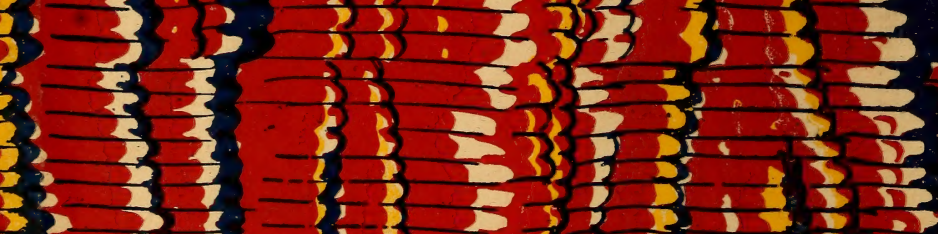

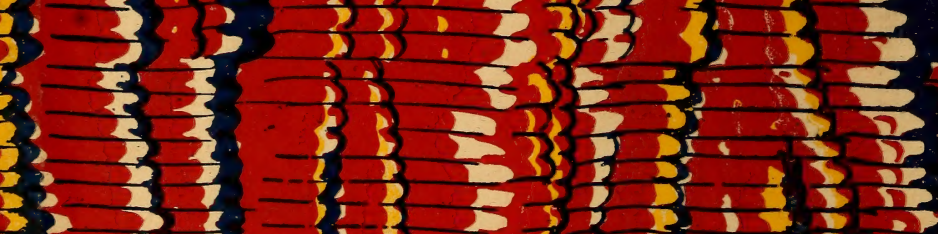

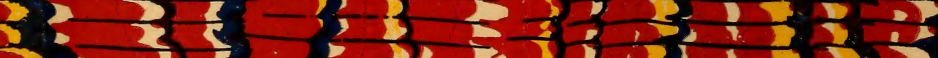

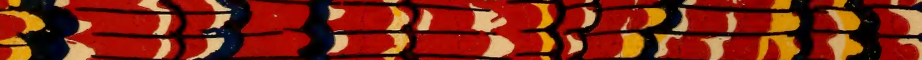
3502535535

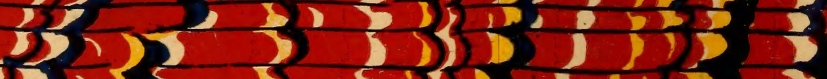

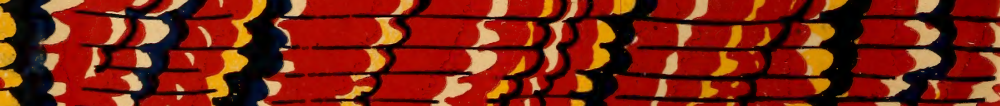

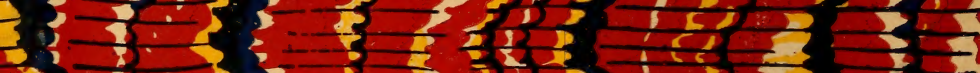

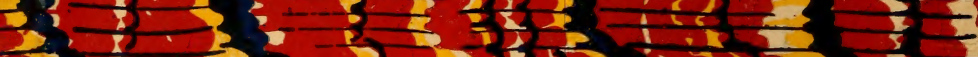

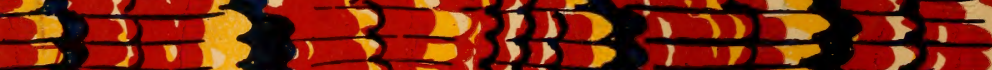

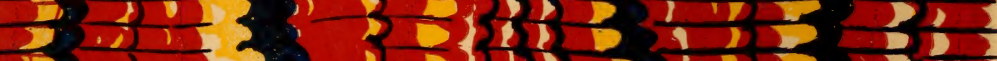

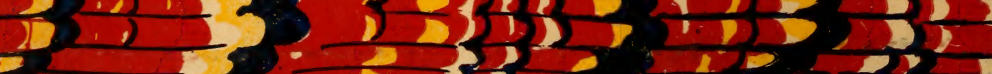
$53-2325+435$ 


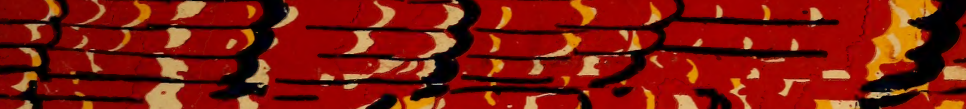

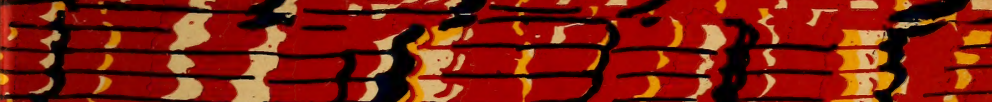
?

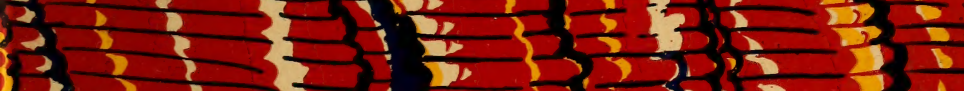

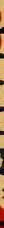

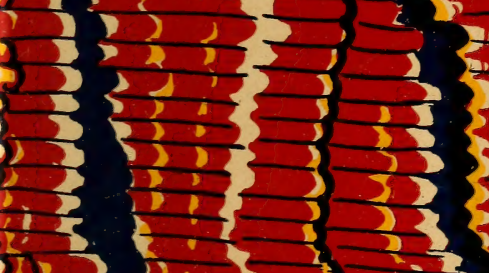

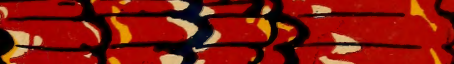

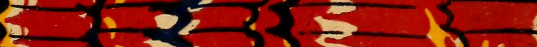

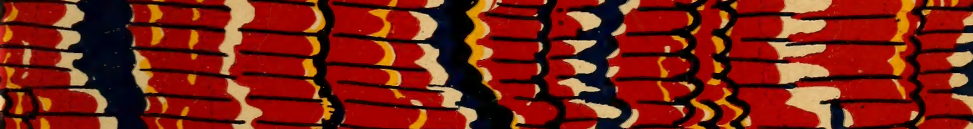

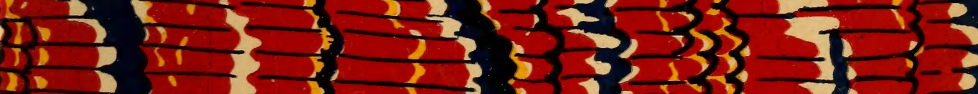

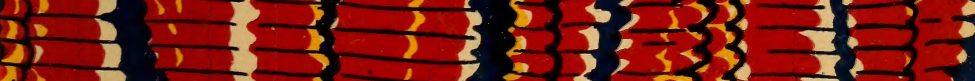

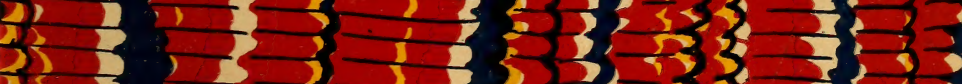

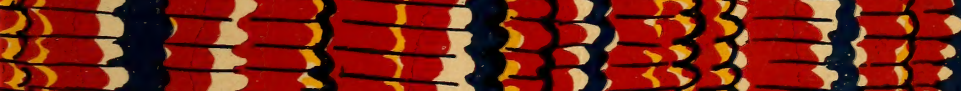

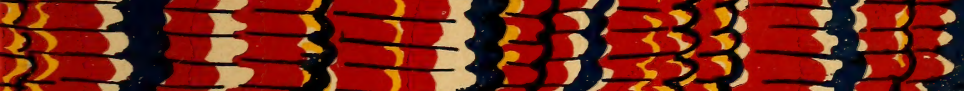

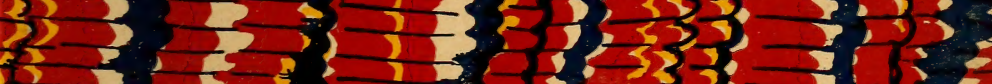

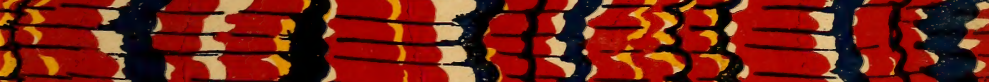

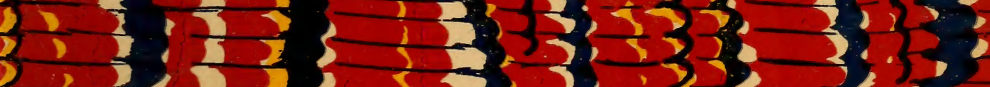

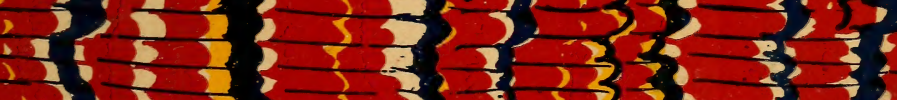

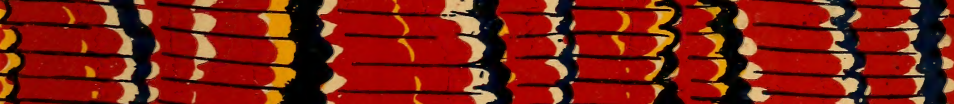

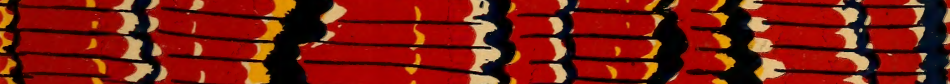
$3,12 \div 3,3=3$ 3,$3 ; \frac{3}{3}, 3,3,3=3$, 3) $323,3,3,35$

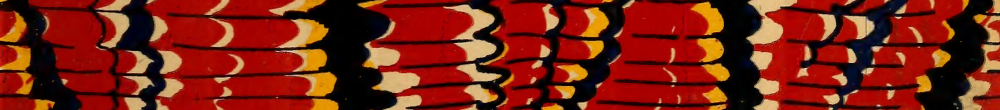

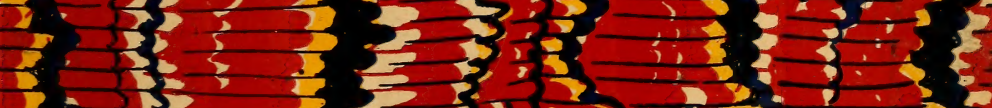

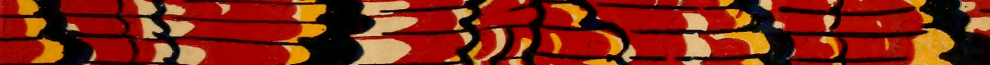

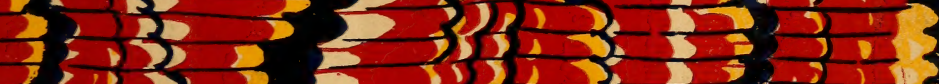

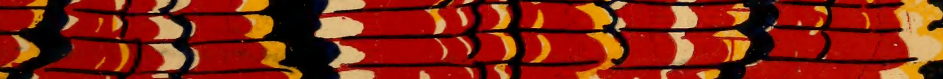

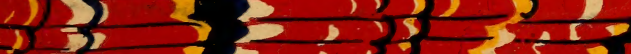

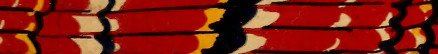
is $5 ; \quad 25$ 3 







\section{1) 3427 \\ tombin}

4.45 



\section{LIST OF CHALCIDITES}





\section{SPECIMENS}

OF

\section{HYMENOPTEROUS INSECTS}

THE COLLECTION

OF THE

\section{BRITISH MUSEUM.}

PART I.—CHALL I D ITES.

PRINTED BY ORDER OF THE TRUSTEES.

LONDON, 1846. 



\section{INTRODUC'TION.}

The principal object of the present Catalogue has been to give a complete list of the specimens of Hymenopterous Insects contained in the collection of the British Museum, indicating at the same time the peculiarities of each, as regards variations in colour, locality, and the source from whence it has been derived.

With this view, the different individuals of each species contained in the collection are indicated by the letters $a, b, c$, \&c., following the name of the species, and its synonymes.

The habitat is given as particularly as the materials possessed by the Museum permit; but it is often impossible to give the place from whence they are derived, except in the most general terms.

Those specimens which have been presented to the Museum have the name of the donor marked immediately after 
the habitat, and in general, the collection from whence the specimen has been purchased, or procured in exchange, is also marked; as it often gives some authenticity to its history.

In the adoption of generic and specific names, it has been thought right to use, wherever it was possible, that which was first applied to the species. As far as regards the specific names, there is comparatively little difficulty in the application of this simple rule; but generic names have been used by different authors in senses so widely different, and the groups which they were meant to designate have been so variously extended or restricted, that it is no easy matter to determine, where several names have been used, which of them ought to be preferred.

If the generic name adopted be different from that first employed for the species after the establishment of the Linnean system of nomenclature, the name under which it was first described follows as a synonyme, and where the Insect has received more than one specific name, those names are also given, as being sufficient for all purposes of identification.

These are followed by a reference to a few of the best original figures of the species.

In those cases where the two sexes of the same species, or any particular individual state or variety belonging to it, have been differently named, the name or figure exclusively appertaining to the state or individual is placed after the indication of the specimen to which it applies. 
This part containing the Chalciditous Insects of this order has been kindly prepared by Francis Walker, Esq., F.L.S., \&c., who has devoted his attention to these Insects, has been the original describer of the greater number of the species, and has presented to the Museum the major part of the collection. The Appendix of this part, written by Mr. Walker, contains the descriptions of the hitherto undescribed species contained in the collection.

The part containing the other families, will follow as soon as they are prepared.

JOHN EDWARD GRAY.

British Museum,

February 12th, 1846. 


\section{LIST OF DONORS.}

Admiralty, (The Lords of the) 2.

Barnston, (George, Esq.) 20, 59, 80.

Brebissun, (M.) 11, 41, 43, 50, 57, 66.

Cooper, (Abraham, Esq., R.A.) 3.

Dale, (J. C., Esq.) 61.

Darwin, (Charles, Esq.) 1-81.

Doubleday, (Edward, Esq.) 3, 4, 7, 10, 20, 32, 35.

Elliott, (Walter, Esq.) 2.

Entomological Club, (The) 1-84.

Haliday, (A. H., Esq.) 8.

Hardwicke Bequest 83.

Maclean, (Dr.) 3.

Morgan, (Rev. D. F.) 5, 22, 23

Passerini, (Signor) 1, 2.

Saunders, (W. W., Esq.) 1, 3.

Smirke, (Lady) 23.

Walker, (Francis, Esq.) 1-96.

Waterhouse, (G. R., Esq.) 71. 


\title{
LIST OF CHAICIDITES.
}

\section{Order. - CHALCIDITES.}

\author{
Family 1. Leucospide.
}

\section{Leucospis, Fabr.}

Levcospis grandis, Klug, Act. Nat. Cur. Berl. vi. 66, 1. L. gigas, Rossi, Faun. Etrusc. ed. Ill. ii. 130. Fonsc. Ann. Sci. Nat. xxvi. 273. L. gallica, Rossi, Mant. 135, 298. L. dorsigera, var. Hochenwarth, Schrift. Berl. Ges. vi. 341, t. 8, f. 1, 2. Christ. Bienen. 225, t. 19, f. 9.

a. Italy. Presented by Signor Passerini.

b. Albania. Presented by F. Walker, Esq.

c. Albania. Presented by W. W. Saunders, Esq.

Le ucospis gigas, Fab. Ent. Syst. ii. 245, 1. L. gallica, De Villers, Ent. iii. 261, t. 8, f. 18. L. dorsigera, Fab. Syst. Ent. 361, 1. Gmel. 2739, 1. Oliv. Enc. Hist. Nat. vii. 352, 1, t. 100, f. 8, 9. Fuessly, Archiv. 2, t. 18, 51,f. 1-10. Leucopsis gigas, Lam. Anim. sans Vert. iv. 151. Dumeril, Dict. des Sci. Nat. xxvi. 169. Cynips nigra, \&c. De la Tourette, Acad. des Sci. ix. 730, f. 1-5. Cynips lugdunæa, Gmel. i. 5, 2653, 26. Sphex dorsigera, Sulzer, Gesch. 196, t. 27, f. 11.

$a-d$. South of France. Presented by the Entomological Club. $e$.

Leucospis Shuckardi, Westwood, Ent. Mag. ii. 214.

a. North America. From Mr. Shuckard's collection.

b. North America. Presented by F. Walker, Esq.

Le ucospis varia, Klug, Act. Nat. Cur. Berl. vi. 67, 3. Leucopsis intermedia, Lam. Anim. sans Vert. iv. 152.

$a, b$. South of France. Presented by the Entomological Club.

c. Italy. From Mr. Birch's collection.

$d, e$. France. From Dr. Leach's collection. 
Leucospis intermedia, Illiger, Edit. Faun. Etr. ii. 130. L. dorsigera, Rossi, Mant. 134, 297. De Villers, Ent. iii. $t$. 8.f. 17. Panz. Faun. Ins. Germ. 15, t. 17.

a. South of France. Presented by the Entomological Club.

Leucospis Biguetina, Jurine, Nouv. Meth. Hym. 307, t. 13, 45. L, gibba, Klug, Act. Nat. Cur. Berl. vi. 68, 5.

a. Italy. Presented by Signor Passerini.

b. Paris. Presented by the Entomological Club.

c. Gibraltar. From Mr. Sowerby's collection.

Levcospis dorsigera, Fab. Ent. Syst. ii. 246, 2. L. dispar? Fab. Syst. Piez. 169,6. L. intermedia, Fonsc. Ann. Sci.Nat. xxvi. 274. L. dubia, Schrank, Fauna Boica, ii. 2, 222, 1981. L. cœlogaster, Schrank, Schrift. Berl. Gesell. i. 301, t. 8, f. 4-6. Leucopsis dorsigera, Lam. Anim. sans Vert. iv. 151. Dumeril, Dict. des Sci. Nat. xxvi. 169, t. 34, f. 2.

$a, b$. Bretagne.

c. Paris. Presented by the Entomological Club.

d. Italy. From Mr. Birch's collection.

$e-j$. Malta. From Mr. Children's collection.

$k$. From Mr. Sowerby's collection.

Le ucospis subnotata, Westwood, Ent. Mag. ii. 215. L. fraterna, Say, Boston .Tourn. Nat. Hist. i. 269.

a. Ohio, North America. Presented by the Entomological Club.

Leucospis miniata? Klug, Symbola Physicce, \&c. Decas 4a.t. xxxvii. $f$. 1 .

a. Voyage of the Blonde. Presented by the Lords of the Admiralty.

Ledcospis atra, Fab. Ent. Syst. Suppl. 259, 3. Syst. Piez. 169, 4. $a, b$. Madras. Presented by Walter Elliott, Esq.

2. Marres, Walker.

Marres Dicomas, Walker, Entomologist, 217.

a. Gambia. From Mr. Rendall's collection.

Fam. 2. Chalcider.

1. Smiera, Spinola.

Smiera nigrifex, Walker, Ent Mag. ii. 22. S. sispes, Spin. Ann. du Mus. d'Hist. Nat. xvii. S. petiolatus, Curt. Brit. Ent. 472. Chalcis sispes, Fab. Mant. Ins. i. 272, 1. Chrysis sispes, Fab. Syst. Ent. 359, $15 . \quad$ Sphex sispes, Fab. Sp. Ins. i. 466, 61. De Villers, Ent. i. 222,6. Vespa dearticulata, Fourc. Ent. Par. ii. 437, $16 . \quad$ Vespa, \&c. Geoff. Ins. Par. ii. 380, 16. Sphex nigrifex, Sulzer, Hist. Ins. 191, 1, f. 27, 1. $a, b$. France.

c. Paris. Presented by the Entomological Club. 
d, e. Georgia, North America. From Mr. Abbot's collection.

$f-h$. England. Presented by A. Cooper, Esq.

$i-k$.

Smiera sispes, Linn. Curtis, Brit. Ent. 472. S. clavipes, Spin. Ann. du Mus. d'Hist. Nat. xvii. Chalcis clavipes, Fab. Mant. Ins. i. 272, 2. C. sispes, Dalm. Act. Kongl. Vet. Acad. Handl. 1820. Sphex fissipes, De Villers, Ent. Lin. iii. 222, 6. Sph. sispes, Linn. Syst. Nat. xii. 2, 943, 13.

a. Albania. Presented by W. W. Saunders, Esq.

b. Paris. Presented by the Entomological Club.

Smiera melanaris, Walker, Ent. Mag. ii. 24. Chalcis melanaris, Dalm. Act. Kongl. Vet. Acad. Handl. 1820. Smiera Macleanii, Curt. Brit. Ent. 472.

$a-c$. England. Presented by Dr. Maclean.

d. Sweden. Presented by F. Walker, Esq.

Smiera xanthostigma, Dalm. Ent. Mag. ii. 25. Chalcis xanthe stigma, Dalm. Act. Kongl. Vet. Acad. Handl. 1820.

a. Sweden. Presented by F. Walker, Esq.

Smiera Side, Walker, Ann. Soc. Ent. France, 2 me Série, i. 145.

a. St. John's Bluff, East Florida. Presented by E. Doubleday, Esq.

Smiera Masus, Walker, Entomologist, 134.

a. From Mr. Sowerby's collection.

b. Brazil.

c. Brazil. Presented by E. Doubleday, Esq.

Smiera Pylas, Walker, Entomologist, 337.

a. Mexico. From Mr. Shuckard's collection.

Smiera Pielus, Walker, Ent. Mag. v. 470.

a. Brazil. Presented by C. Darwin, Esq.

Smiera Dares, Walker, Entomologist, 338.

a. Brazil. From Mr. Shuckard's collection.

Smiera punctata. S. subpunctata, Lansdown Guildiny, MSS.

Walker, Ent. Mag. ii. 25, v. 469. Chalcis fasciata, Oliv. Enc.

Meth. Hist. Nat. Ins. v. 439. C. punctata, Fab. Ent. Syst. ii. 196, 5. Sphex punctata, Fab. Spec. Ins. i. 446, 22.

a. Brazil. Presented by C. Darwin, Esq.

$b, c$. St. Vincent's Island, West Indies. Presented by the Entomological Club.

d. Brazil. From Mr. Shuckard's collection.

e. Jamaica. From Mr, Gosse's collection.

f. Brazil.

$g, h$. From Mr. Sowerby's collection. 
Smiera fulvescens, Lansdown Guilding, MSS. Walker, Ent. Mag. ii. 25.

$a, b$. St. Vincent's Island, West Indies. Presented by the Entomological Club.

c. United States. Presented by E. Doubleday, Esq.

Smiera maculata, Walker, Entomologist, 217. Chalcis maculata, Fab. Ent. Syst. ii. 198, 11.

a. New York, North America. Presented by E. Doubleday, Esq.

\section{Epitranus, Walker.}

Epitranus fulvescens, Lansdown Guilding, MSS. Walker Ent. Mag. ii. 26.

$a-c$. St. Vincent's Island, West Indies. Presented by the Entomological Club.

Epitranus Teleute. Smiera Teleute, Walker, Ent. Mag. v. 471. $a, b$. Hobart Town, Van Diemen's Land. Presented by C. Darwin, Esq.

3. Chalcis, Fab.

Chalcis flavipes, Fab. Ent. Syst. ii. 197, 10. Syst. Piezat. 167, 32. a. Paris. Presented by the Entomological Club. $b$.

Chalcis femorata, Panz. Faun. Ins. Germ. Fasc. 84, f. $16 . \quad$ C. flavipes, Latr. Gen. Crust. et Ins. iv. 26.

a. Paris. Presented by the Entomological Club.

b. Germany. From Dr. Klug's collection.

Chalcis distinguenda, De Laporte, MSS. Walker, Ent. Mag. ii. 29.

$a-c$. South of France. Presented by the Entomological Club.

d. Malta. From Mr. Children's collection. e.

Chalcis tibialis, De Laporte, MSS. Walker, Ent. Mag. ii. 29.

C. intermedia? Nees ab Ess. Hym. Ich. aff. Mon. ii. 29.

a. Paris. Presented by the Entomological Club.

Chalcis annulipes, De Laporte, MSS. Walker, Ent. Mag. ii.

29. C. flavipes, Fab. Ent. Syst. ii. 197, 10.

a. St. Vincent's Island, West Indies. Presented by the Entomological Club.

b. Georgia, North America. From Mr. Abbot's collection.

c. From Mr. Sowerby's collection.

Chatcis cingulata, De Laporte, MSS. Walker, Ent. Mag. ii. 30. $a-c$. Paris. Presented by the Entomological Club. 
Chalcis minuta, Fab. Syst. Piezat. 165, 24, 23. C. femorata, Dalm. Kongl. Vet. Acad. Handl. 1820. Brachymeria minuta, Westwood, Lond. \& Edin. Phil. Mag. 3rd Series, i. 127. Vespa femoralis, Fourc. Ent. Par. ii. 437, 15. Vespa, \&c. Geoff. Ins. ii. 380, 15. V. minuta, Linn. Syst. Nat. 2, 952, 28. $a-d$. South of France. Presented by the Entomological Club. $e-g$.

h. Georgia, N. America. From Mr. Abbot's collection. $i$.

Chalcis podagrica, Fab. Syst. Piezat. 166, 24. C. minuta, var. Rossi, Faun. Etr. App. 99. C. femorata, fem. Dalm. Kongl. Vet. Acad. Handl. 1820. Nees ab Ess. Hym. Ich. aff. Mon. ii. 28.

$a, b$. South of France. Presented by the Entomological Club.

Chalcis parvula, De Laporte, MSS. Walker, Ent. Mag. ii. 32. C. minuta, Dalm. Kongl. Vet. Acad. Handl. 1820. Nees ab Ess. Hym. Ich. aff. Mon. ii. 30. C. pusilla, Rossi, Faun. Etr. Mant. 272, 6. Spin. Ins. Lig. fasc. iii. 164, 7. $a-e$. South of France, Presented by the Entomological Club. f. France.

Chalcis Orseis, Walker, Entomologist, 338. $a, b$. Brazil. From Mr. Shuckard's collection.

Chalcis Mnestor, Walker, Entomologist, 219. C. villosa? Oliv. Enc. Meth. Hist. Nat. Ins. v. 438.

a. Brazil. From Mr. Shuckard's collection.

Cha lcts Cabira, Walker, Ent. Mag. v. 472.

a. Charles's Island, Galapagos. Presented by C. Darwin, Esq.

Chalcis Phya, Walker, Ent. Mag. v. 471. $a, b$. Sydney, New South Wales. Presented by C. Darwin, Esq.

Chalcis — See Appendix.

a. Sierra Leone. Presented by the Rev. D. F. Morgan.

Chalcis - See Appendix.

a. Sierra Leone. Presented by the Rev. D. F. Morgan.

Chalcis Amphissa. See Appendix. a. Nepal.

Chalcis Xerxena. See Appendix. a. Philippine Islands. From Mr. Wond's collection.

Chalcis Alexion. See Appendix.

a. Italy. 


\section{Phasgonophora, Westwood.}

Phasgonophora sulcata, Westw. Griff. Transl. Cuv. Régne An. xv. $432, t .77, f .2$.

a. Georgia, North America. From Mr. Abbot's collection.

\section{Haltichella, Spinola.}

Haltichella pusilla, Spin. Ann. Mus. Hist. Nat. xvii. Chalcis pusilla, Fab. Syst. Piezat. 167, 29.

a. Paris. Presented by F. Walker, Esq.

Haltichella bispinosa, Spin. Ann. Mus. Hist. Nat. xvii. Hockeria bispinosa, De Lap. MSS. Walker, Ent. Mag. ii. 35. Chalcis bispinosa, Fab. Syst. Piezat, 166, 28. Fonsc. Ann. Sci. Nat. xxvi. $279,9$.

$a, b$. France.

Haltichella bifasciata. Hockeria bifasciata, De Lap. MSS. Walker, Ent. Mag. ii. 35. Chalcis bimaculata, Fonsc. Ann. Sci. Nat. xxvi. 280, 11.

a. Paris. Presented by F. Walker, Esq.

b. France.

Haltichella Dargelasii, Spin. Class. Dipl. Ann. Mus. vii. 148. Hockeria nigra, De Lap. MSS. Walker, Ent. Mag. ii. 36. Chalcis clavipes, Rossi, Mant. 272, 2. Faun. Etr. ed. Ill. ii. 86, 803. C. rufitarsis, 1ll. Rossi, Faun. Etr. ii, 87. C. Dargelasii, Latr. Hist. Nat. des Crust. \&c. xiii. 221, 6.

a. Paris. Presented by the Entomological Club.

Haltichella nigripes. Hockeria nigripes, De Lap. MSS. Walker, Ent. Mag. ii. 36. Chalcis nigripes, Fonse. Ann. Sci. Nat. xxvi. 280, 10.

a. Paris. Presented by the Entomological Club.

Haltichella rufipes. Hockeria rufipes, De Lap. MSS. Walker, Ent. Mag. ii. 36. Chalcis rufipes, Oliv. Ent. Meth. v. 440, 11. Latr. Hist. Nat. des Crust. \&c. xiii. 221, 5. Spin. Ins. Lig. fasc. i. $63 . \quad$ C. armata, Panz. Krit. Rev. 98. Spin. Ins. Lig. fasc. iii. 163, 5. Latr. Gen. Crust. \&c. iv. 26. Dalm. Kongl. Vet. Acad. Handl. 1820.

a. Paris. Presented by the Entomological Club.

$b$. Sweden. Presented by F. Walker, Esq.

c. From Mr. Shuckard's collection.

Haltichella unicolor. Hockeria unicolor, Walker, Ent. Mag. ii. 37 .

a. South of France. Presented by the Entomological Club.

b. France. 
Haltichella Mitys. See Appendix.

a. Isle of France.

Haltichella Amage. See Appendix.

a.

Haltichella Proxenus, Walker, Mon. Chal. ii. 8.

a. Hobart Town, Van Diemen's Land. Presented by C. Darwin, Esq.

b. Sydney, New South Wales: Presented by C. Darwin, Esq.

Haltichella Eracon, Walker, Ent. Mag. v. 473.

a. Hobart Town, Van Diemen's Land. Presented by C. Darwin, Esq.

Haltichella Nyssa, Walker, Ent. Mag. v. 474.

a. Sydney, New South Wales. Presented by C. Darwin, Esq.

Haltichella Dexius, Walker, Ent. Mag. v。 473.

a. King George's Sound, Australia. Presented by C. Darwin, Esq.

Haltichella Onatas, Walker, Ann. Soc. Ent. France, 2 me Série, i. 146.

$a-e$. St. John's Bluff, Florida. Presented by E. Doubleday, Esq. Haltichella Xanticles, Walker, Ann. Soc. Ent. France, $2 m e$ Série, i. 146.

$a-c$. St. John's Bluff, Florida. Presented by E. Doubleday, Esq.

6. Dirhinus, Dalman.

Dirhinus hesperidum, Nees ab Ess. Hym. Ich. aff. Mon. ii. 36. D. cornigerus, Ent. Mag. ii. 38. Chalcis hesperidum, Rossi, Faun. Etr. App. 100, t. 7, f. D. C. cornigera, Jur. Hym. 315, t. 13, 47. Latr. Gen. Crust. \&c. iv. 26. Spin. Ins. Lig. fasc. iii. 164, 8. Chrysis hesperidum, Ill. Rossi, Faun. Etr. ii. $126,832$.

a. Paris. Presented by the Entomological Club.

Dirhinus Anthracia. See Appendix.

a. Philippine Islands. From Mr. Wood's collection.

7. Notaspis, Walker.

Notaspis formiciformis, Lansdown Guilding, MSS. Walker, Ent. Mag. ii. 38.

a. St. Vincent's Island, West Indies. Presented by the Entomological Club.

\section{Agamerion, Haliday.}

Agamerion Gelo, Haliday, Trans. Ent. Soc. iii. 298. Miscogaster Gelo, Walk. Mon. Chal. ii. 27.

a. Sydney, New South Wales. Presented by C. Darwin, Esq. 


\section{Fam. 3. Eurytomide.}

\section{Eurytona, Illiger.}

Eurytoma plumata, mas, Illiger, Rossi, Faun. Etr. ii. 127, 853. E. Serratulæ, Latr. Gen. Crust. et Ins. iv. $27 . \quad$ Bouchè, Nat. der Ins. i. 166, 58. E. verticillata, Ent. Mag. i. 23. E. tibialis, Zett. Boh. Kongl. Vet. Acad. Handl. 1835, 232. Chrysis plumata, Rossi, Faun. Etr. C. Adonidum, fem. Rossi, Faun. Etr. ed. Ill. ii. 128. Cynips Serratulæ, Fab. Ent. Syst. Supp. 214, 15-16. C. Adonidum, fem. Rossi, Faun. Etr. App. 75. C. compressa, fem. Fab. Ent. Syst. Suppl. 213, 1. C. plumata, mas, Rossi, Faun. Etr. App. 79, t. 7, f. F. C. aterrima, Schrank, Enum. Ins. Austr. 645. De Vill. Ent. Lin. iii. 75, 19. Diplolepis Adonidum, fem. Spin. Ins. Lig. fasc. iv. t. 3, f. 10. Decatoma Adonidum, fem. Spin. Ann. du Mus. vii. 151. Eucharis compressa, fem. Fab. Syst. Piez. 157, 3. E. Serratulæ, mas, Panz. Krit. Rev 69.

a. England. Presented by F. Walker, Esq.

b. South of France. Presented by F. Walker, Esq.

c. France.

Eurytoma longipennis, Walker, Ent. Mag. i. 23.

a. Paris. Presented by the Entomological Club.

Eurytoma tumida, Walker, Ann. Nat. Hist. xiv. 18.

a. England. Presented by F. Walker, Esq.

Eurytoma platyptera. Systole platyptera, Walker, Ent. Mag. ii. 158.

a. Ireland. Presented by A. H. Haliday, Esq.

Eurytoma curta, Walker, Ent. May. i. 24, 5 .

$a$. England. Presented by F. Walker, Esq. $b$.

Eurytoma Abrotani, Illiger, Rossi, Faun. Etr. ii. 128. Latr. Gen. Crust. et Ins. iv. 27. Chalcis Abrotani, Panz. Faun. Germ. 76, 14. Eucharis Abrotani, Panz. Krit. Rev. 97. Cynips atra? Enc. Meth. Hist. Nat. Ins. v. 781, 7. Müll. Faur. Frid. 595 ?

a. England. Presented by F. Walker, Esq.

b. France.

Eurytoma apicalis, Walker, Ent. Mag. i. 24. E. Rosæ? Nees ab Ess. Hym. Ich. affin. Mon. ii. 415.

a. England. Presented by F. Walker, Esq.

Eurytoma nodularis, Boheman, Kongl. Vet. Acad. Handl. 1835, 230. E. Argele, Walker, Ann. Nat. Hist. xiv. 19.

a. England. Presented by F. Walker, Esq.

b. Sweden. Presented by F. Walker, Esq. 
Eurytoma collaris, Walker, Ent. Mag. i. 24, 6.

a. England. Presented by F. Walker, Esq.

Eurytoma Scultenua, Walker, Ann. Nat. Hist. xiv. 20. E. scabra? Foerster, Monog. Pteromalinen, i. 31.

a. England. Presented by F. Walker, Esq.

Euryтoma Micipsa, Walker, Ann. Nat. Hist. xiv. 21.

a. England. Presented by F. Walker, Esq.

Eurytoma brevicollis, Haliday, MSS. Walker, Ent. Mag. iv. 24. a. England. Presented by F. Walker, Esq.

Eurytoma annulipes, Walker, Ent. Mag. i. 25, 9. E. pubicornis, Boh. Kongl. Vet. Acad. Handl. 1835, 247. E. punctulata? Foerster, Mon. Pteromal. i. 31. E. atra? Nees ab Ess. Hym. Ich. aff. Mon. ii. 42.

a. England. Presented by F. Walker, Esq.

b. Sweden. Presented by F. Walker, Esq.

c.

Eurytoma gracilis, Walker, Ent. Mag. i. 25, 8. E. minor, Dal. Boh. Kongl. Vet. Acad. Handl. 1835, 235.

a. Sweden. Presented by F. Walker, Esq.

Eurytoma minuta, Walker, Ent. Mag. i. 25, $10 . \quad$ E. pumila? Foerster, Mon. Pteromal. 1. 31.

a. France. Presented by F. Walker, Esq.

Eurytoma rufipes, Walker, Ent. Mag. i. 25, 11. Dalm. Boh. Kongl.Vet. Acad. Handl. 1835, 235.

a. England. Presented by F. Walker, Esq.

b. Sweden. Presented by F. Walker, Esq.

Eurytoma æthiops, Boh. Kongl. Vet. Acad. Handl. 1835, 240. E. Sittace, Walker, Ann. Nat. Hist. xiv. 19.

a. England. Presented by F. Walker, Esq.

b. Sweden. Presented by F. Walker, Esq.

Eurytoma appendigaster, Boh. Kongl. Vet. Acad. Handl. 1835, 231. Dalm. Kongl. \& c. 1820. Pteromalus appendigaster, Swed.Kongl. \&c. 1795.

a. Sweden. Presented by F. Walker, Esq.

Eurytoma flavimana, Boh. Kongl. Vet. Acad. Handl. 1835, $23 \%$.

a. Sweden. Presented by F. Walker, Esq.

Eurytoma nigrita, Boh. Kongl. Vet. Acad. Handl. 1835, 238. Dalm. Kongl. \&c. 1820. Pteromalus nigritus, Swed. Kongl. \&c. 1795. Eurytoma flavipes, De Laporte, MSS. Walker, Ent. Mag. ii. 155.

a. Sweden. Presented by F. Walker, Esq.

Eurytoma Salicis, De Laporte, MSS. Walker, Ent. Mag. ii. 155. E. gibba, Boh. Kongl. Vet. Acad. Handl. 1835, 244.

a. Sweden. Presented bv F. Walker, Esq. 
Eurytoma squamea, De Laporte, MSS. Walker, Ent. Mag. ii. 154.

a. France. Presented by the Entomological Club.

Eurytoma acuminata, De Laporte, MSS. Walker, Ent. Mag. ii.

154. E. morio, Boh. Kongl. Vet. Acad. Handl. 1835, 241.

a. Sweden. Presented by F. Walker, Esq.

Eurytoma Cretheis, Walker, Ann. Soc. Ent. France, 2 me Serie, i, 150.

a. St. John's Bluff, Florida. Presented by E. Doubleday, Esq.

Eurytoma Hecale, Walker, Ann. Soc. Ent. France, 2me Serie, i. 151.

a. St. John's Bluff, Florida. Presented by E. Doubleday, Esq.

Eurytoma Abatos, Walker, Ann. Soc. Ent. France, 2 me Serie, i. 152.

a. St. John's Bluff, Florida. Presented by E. Doubleday, Esq. Eurytoma Teredon, Walker, Ann. Soc. Ent. France, 2me Serie,

i. 153. E. studiosa? Say, Boston Journ. Nat. Hist. i. 272.

a. St. John's Bluff, Florida. Presented by E. Doubleday, Esq.

Eurytoma Pythes, Walker, Ann. Ent. Soc. France, $2 m e$ Serie, i. 154.

$a$. St. John's Bluff, Florida. Presented by E. Doubleday, Esq. Eurytoma Iphis, Walker. See Appendix.

a. St. John's Bluff, Florida. Presented by E. Doubleday, Esq.

Eurytoma Pidytes, Walker, Mon. Chal. ii. 2.

a. Hobart Town, Van Diemen's Land. Presented by C. Darwin, Esq.

b. King George's Sound, Australia. Presented by C. Darwin, Esq.

Eurytoma Tellis, Walker, Mon. Chal. ii. 2.

a. Sydney, New South Wales. Presented by C. Darwin, Esq.

Eurytoma Aretheas, Walker, Mon. Chal. ii. 3.

a. King George's Sound, Australia. Presented by C. Darwin, Esq.

Eurytoma Menon, Walker, Mon. Chal. ii. 62.

a. Bahia, Brazil. Presented by C. Darwin, Esq.

Eurytoma Euclus, Walker, Mon. Chal. ii. 62.

a. Bahia, Brazil. Presented by C. Darwin, Esq.

Eurytoma Philager. Decatoma Philager, Walker, Mon. Chal. ii. 81 .

a. Isle of Chiloe, Chili Presented by C. Darwin, Esq. 
2. Systole, Walker.

Srstole albipennis, Walker, Ent. Mag. i. 22. Eurytoma brevicornis, Boh. Kongl. Vet. Acad. Handl. 1835, 245. E. nitida, Walker, Ent. Mag. i. 25.

a. England. Presented by F. Walker, Esq.

b. Sweden. Presented by F. Walker, Esq.

3. Isosoma, Walker.

Isosoma verticillata. I. longulum, Walker, Ent. Mag. i. 14. I. fumipenne, var. Ent. Mag. is 15 . I. simile, var. Ent. Mag. i. 16. Eurytoma verticillata, Ill. Rossi, Faun. Etr. ii. 128. Diplolepis verticillata, Fab. Syst. Piezat. 153, 23. Spin. Ins. Lig. fasc. i. 62, 6. Ichneumon verticillatus, Fab. Ent. Syst. Suppl. 232, 235, 236.

a. England. Presented by F. Walker, Esq.

b. Sweden. Presented by F. Walker, Esq.

$c$. France. Presented by M. Brebisson.

Isosoma crassicorne, Walker, Ent. Mag. i. 15. a. England. Presented by F. Walker, Esq. Isosorra longicorne, Walker, Ent. Mag. i. 17. a. England. Presented hy F. Walker, Esq.

Isosoma angustipenne, Walker, Ent. Mag. i. 16.

a. England. Presented by F. Walker, Esq.

Isosoma brevicorne, Walker, Ent. Mag. i. 16. a. England. Presented by F. Walker, Esq.

Isosoma hyalipenne, Walker, Ent. Mag. i. 17. Eurytoma afra, Boh. Kongl. Vet. Acad. Handl. 1835, 242.

a. England. Presented by F. Walker, Esq.

b. Sweden. Presented by F. Walker, Esq.

Isosoma minor, Walker, Ent. Mag. i. 18. I. elongatum, var. Walker, Ent. Mag. i. 18. Eurytoma pusilla, Boh. Kongl. Vet. Acad. Handl. 1835, 232.

a. England. Presented by F. Walker, Esq.

b. Sweden. Presented by F. Walker, Esq.

Iososma petiolatum, Walker, Ent. Mag. i. 19. a. England. Presented by F. Walker, Esq. Isоsом с cornutum, Walker, Ent. Mag. i. 19. a. England. Presented by F. Walker, Esq. Isosoma breve, Walker, Ent. Mag. i. 18. a. England. Presented by F. Walker, Esq. Isosoma angustatum, Walker, Ent. Mag. i. 20. a. England. Presented by F. Walker, Esq. 
Isosoma dissimile, Walker, Ent. Mag. i. 20. Presented by F. Walker, Esq.

Isosoma fulvicolle, Walker, Ent. Mag. i. 21. a. England. Presented by F. Walker, Esq.

Isosoma flavicolle, Walker, Ent. Mag. ii. 153.

a. England. Presented by F. Walker, Esq.

Isosoma depressum, Walker, Ent. Mag. i. 21.

a. England. Presented by F. Walker, Esq.

Isosoma lineare, Walker, Ent. Mag. i. 22. Eurytoma longula, Boh. Kongl. Vet. Acad. Handl. 1835, 248.

a. England. Presented by F. Walker, Esq.

b. Sweden. Presented by F. Walker, Esq.

Isosoma attenuatum, Walker, Ent. Mag. i.22. Eurytoma guttula, Boh. Kongl. Vet. Acad. Handl. 1835, 250.

a. Sweden. Presented by F. Walker, Esq.

Isosoma Laothoe, Walker, Ann. Nat. Hist. xii. 103.

a. England. Presented by F. Walker, Esq.

Isosoma brevipenne, Walker, Ent. Mag. iv. 24.

a. England. Presented by F. Walker, Esq.

Isosoma Hordei, Walker, Ann. Nat. Hist. xii. 103. Eurytoma Hordei, Harris. New England Farmer, July, 1830.

a. Philadelphia, North America. Presented by F. Walker, Esq. Isosoma Volux. Eurytoma Volux, fem. Walker, Mon. Chal. ii. 3. E. Eleuther, mas, Mon. Chal. ii. 3.

a. Hobart Town, Van Diemeu's Land. Presented by C. Darwin, Esq.

b. King George's Sound, Australia. Presented by C. Darwin, Esq. Isosoma Olbus. Eurytoma Olbus, Walker, Mon. Chal, ii. 4. a. Sydney, New South Wales. Presented by C. Darwin, Esq. Isosoma Ravola, Walker, Mon. Chal.ii. 4.

a. Hobart Town, Van Diemen's Land. Presented by C. Darwin, Esq.

Isosoma Oritias, Walker, Mon. Chal. ii. 5.

a. King George's Sound, Australia. Presented by C. Darwin, Esq.

Isosoma Egesta, Walker, Entomologist, 334. a. Europe.

4. Decatoma, Spinola.

Decatoma semifasciata, De Laporte, Ent. Mag. ii. 156.

a. France. Presented by the Entomological Club. 
Decatoma biguttata, Walker, Ent. Mag. i. 26. D. Cooperi, var.? Curtis, Brit. Ent. 345. Eurytoma biguttata, Nees ab Ess. Hym. Ich. aff. Mon. ii. 44, 1. Boh. Kongl. Vet. Acad. Handl. 1835, 227. Zett. Ins. Lap. 419. Pteromalus biguttatus, Swed. Kongl. \&c. 1795, iv. 216, 5. Eurytoma Cooperi, Guerin, Icon. Règne. Anim. t. 67, f. 11, texte 416.

a. England. Presented by F. Walker, Esq.

b. Sweden. Presented by F. Walker, Esq.

Decatoma obscura, Walker, Ent. Mag. i. 26.

a. England. Presented by F. Walker, Esq.

b. South of France. Presented by F. Walker, Esq.

Decatoma immaculata, Walker, Ent. Mag. i. 27.

a. England. Presented by F. Walker, Esq.

Decatoma variegata, Walker, Ent. Mag. i. 28. Eurytoma signata, Nees ab Ess. Hym. Ich. aff. Mon. ii. 43.

a. England. Presented by F. Walker, Esq.

Deсатома flavicollis, Walker, Ent. Mag. ii. 156. Eurytoma xanthomelas, Boh. Kongl. Vet. Acad. Handl. 1835, 226.

a. England. Presented by F. Walker, Esq.

b. Sweden. Presented by F. Walker, Esq.

Decatoma mellea, Walker, Ent. Mag. i.27. Eurytoma concinna, Boh. Kongl. Vet. Acad. Handl. 1835, 225. Cynips binotata et rufa? Boyer de Fonsc. Ann. Sci. Nat. 1832, 289, 290, 16, 17. Chrysolampus? binotatus et rufus, Nees, Hym. Ich. aff. ii. 427.

a. England. Presented by F. Walker, Esq.

b. Sweden. Presented by F. Walker, Esq.

Decatoma unicolor, Walker, Ent. Mag. i. 28.

a. England. 'Presented by F. Walker, Esq.

Decatoma cynipsea. D. aspilus, Walker, Ent. Mag. iv. 25. Eurytoma cynipsea, Boh. Kongl. Vet. Acad. Handl. 1835, 229.

a. England. Presented by F. Walker, Esq.

b. Sweden. Presented by F. Walker, Esq.

Decatova minuta Walker, Ent. Mag. i. 28. Presented by F. Walker, Esq.

Decatoma Oretilia, Walker, Ann. Nat. Hist. xii. 46.

a. St. Vincent's Island, West Indies. Presented by F. Walker, Esq.

Decatoma Diphilus, Walker, Mon. Chal. ii. 63.

a. Bahia, Brazil. Presented by C. Darwin, Esq. 


\section{Fam. 4. Torymide.}

\section{$\therefore$ 1. Megastigmus, Spinola.}

Megastigmus collaris, Boheman, Kongl. Vet. Acad. Handl. 1838, 332. M. transversus, Walker, Ent. Mag. i. 117. Torymus punctum? Foerster Mon. Pterom. i. 31.

a. England. Presented by F. Walker, Esq.

b. Sweden. Presented by F. Walker, Esq.

Megastigmus dorsalis, Boh. Kongl. Vet. Acad. Handl. 1833, 334,

Torymus chloronotus, Dalm. Kongl. \&c. 1820. Cinips dorsalis, Fonsc. Ann. Sci. Nat. xxvi. 282. Diplolepis dorsalis, Fab. Syst. Piezat. 151, $11 . \quad$ Ichneumon dorsalis, Fab. Ent. Syst. Suppl. 231, 218.-Coqueb. Illustr. Icon. i. t. 5, f. 3. ? Reaum. iii. $t .39, f .11$.

a. England. Presented by F. Walker, Esq.

b. Sweden. Presented by F. Walker, Esq.

Megastigmus bipunctatus, Boheman, Kongl. Vet. Acad. Handi. 1833, 331. Torymus pictus? Foerster, Mon. Pterom, i. 31.

a. Sweden. Presented by F. Walker, Esq.

Megastig mus Drances, Walker, Mon. Chal. ii. 5.

a. Hobart Town, Van Diemen's Land. Presented by C. Darwin, Esq.

Megastigmus Borus, Walker, Mon. Chal. ii. 5.

a. Hobart Town, Van Diemen's Land. Presented by C. Darwin, Esq.

Megastigmus Iamenus. Walker, Mon. Chal. ii. 6.

a. Hobart Town, Van Diemen's Land. Presented by C. Darwin, Esq.

\section{Palmon, Dalman.}

Palmon pachymerus. Priomerus pachymerus, Walker, Ent. Mag.

i. 118. Entomologist, pl. F. fig. 3.

a. South of France. Presented by the Entomological Club. Palmon Olenus, Walker, Mon. Chal. ii. 7.

a. Sydney, New South Wales. Presented by C. Darwin, Esq.

3. Torvmus, Dalman.

Tonymus caliginosus, Walker, Ent. Mag. i. 118. Entomologist, pl. I. fig. 4 .

a. South of France. Presented by the Entomological Club.

Torymus Phormio, Walker, Ann. Nat. Hist. x. 113.

a. Valparaiso, Chili. Presented by C. Darwin, Esq.

4. Monodontomerus, Westwood.

Monodontomerus stigma, Walker, Ent. Mag. ii. 158. Calli- 
mome stigma, Walker, Ent. Mag. i. 139. Cynips stigma, Fonse. Ann. Sci. Nat. xxvi. 289. Diplolepis stigma, Fabr. Syst. Piezat. 152, 21. Ichneumon stigma, Fab. Ent. Syst. ii. $188,229$.

a. France. Presented by the Entomological Club.

Monodontomerus dentipes. Torymus dentipes, Boheman, Kongl. Vet. Acad. Handl. 1833, 335. Monodontomerus obscurus, Westwood, Lond. \& Edin. Phil. Mag. 3rd series, ii. 443. Callimome pubescens, Walker, Ent. Mag. i. 138. Torymus cupreus, Nees ab Ess. Hym. Ich. aff. Mon. ii. 67. Diplolepis cuprea, Spinola, Ins. Lig. fasc. iv. 212. 1xix. t. 3, f. 11 .

a. England. Presented by F. Walker, Esq.

b. Sweden. Presented by F. Walker, Esq.

c. South of France. Presented by the Entomological Club.

5. Diomorus, Walker.

Diomorus armatus. Torymus armatus, Boh. Kongl. Vet. Acad. Handl. 1833, 337. T. chrysis, Nees ab Ess. Hym. Ich. aff. Mon. ii. 67. Diomorus nobilis, Walker, Ent. Mag. ii. 159. Entomologist, pl. I. fig. 5 .

a. Sweden. Presented by F. Walker, Esq.

6. Callimome, Spinola.

Callimome cynipedis, Walker, Ent. Mag. i. 119. Torymus fulgidus, Boh. Kongl. Vet. Acad. Handl. 1833, 345. T. fulgens, Nees, Hym. Ich. aff. Mon . ii. 61. Diplolepis cynipedis, Fab. Syst. Piezat. 152, 17. Ichneumon cynipedis, Linn. Syst. Nat. ii. 939, 68. Faun. Suec. 409, 1639. Fab. Ent. Syst. ii. 187, 223. I. Erucarum, Schrank, Enum. Ins. Austr. 375.

a. England. Presented by F. Walker, Esq.

b. Sweden. Presented by F. Walker, Esq.

Callimome nobilis. Torymus nobilis, Boh. Kongl. Vet. Acad. Handl. 1833, 339. Callimome Roboris, Walker, Ent. May. i. 120. C. subterraneus, Curtis, Brit. Ent. 552. Cinips ventralis, Fonsc. Ann. Sci. Nat. 1832, 286. Torymus conjunctus, Nees, Hym. Ich. aff. Mon. ii. 63 ?

a. England. Presented by F. Walker, Esq.

b. Sweden. Presented by F. Walker, Esq.

Callimome quadricolor, Walker, Ent. Mag. i. 120. Torymus affinis, Foerster, Mon. Pterom. i. 31.

a. England. Presented by F. Walker, Esq.

Calimome cingulatus. C. Geranii, Curtis, Walker, Ent. Mag. i. 121. Torymus cynipedis, Boh. Kongl. Vet. Acad. Handl. 1833, 342. T. cingulatus, Nees, Hym. Ich. aff. Mon. ii. 62, 6. Diplolepis cynipedis, Spin. Ins. Lig. fasc. iv. 213. Torymus medius, Foerster, Mon. Pterom. i. 31. 
a. England. Presented by F. Walker, Esq.

b. Sweden. Presented by F. Walker, Esq.

Callimome Bedeguaris, Spinola, Ann. Mus. vii. 148. Walker. Ent. Mag. i. 121. Torymus Bedeguaris, Boh. Kongl. Vet. Acad. Handl. 1833, 350. Cinips Bedeguaris, Latr. Hist. Nat. xiii. 225, 10. Oliv. Enc. Meth. Fonsc. Ann. Sci. Nat. xxvi. 283. Misocampus Bedeguaris, Latr, \&c. Pteromalus Bedeguaris, Swed. Kongl. \&c. 1795. Diplolepis Bedeguaris, Fab. Syst. Piezat. 150, 6. Ichneumon Bedeguaris, Lin. Syst. Nat. ii. 993, 63. Faun. Suec. 408, 1634. Fab. Ent. Syst. ii. 185, 215. Geoff. Ins, ii. 296, 1. Roes. Ins. iii. t. 53, f. F. A. Act. Nat. Cur. dec. ii. ann. ii. obs. 10. Reaum. Ins. iii. t. 41, f. 13, 14. Rossi, Faun. Etr. 279, 291. F. Sp. 438, 110. De Geer, Ins. ii. 186, 10.

a. England. Presented by F. Walker, Esq.

Calcimome Hederæ, Walker, Ent. Mag. i.123. Torymus compressus? Foerster, Mon. Pterom. i. 32.

a. England. Presented by F. Walker, Esq.

Callimome elegans. Torymus elegans, Boh. Kongl. Vet. Acad. Handl. 1833, 352. Callimome varians, Walk. Ent. Mag. i. 122. Torymus pubescens? Foerster, Mon. Pterom, i. 32.

a. England. Presented by F. Walker, Esq.

b. Sweden. Presented by F. Walker, Esq.

e. Paris. Presented by the Entomological Club.

Callimome amœnus. Torymus amœnus, Boh. Kongl. Vet. Acad. Handl. 1833, 348. Callimome scutellaris, Walker, Ent. May. i. 123. Torymus auronitens? Foerster, Mon. Pterom. i. 32.

a. Sweden. Presented by F. Walker, Esq.

Callimome Druparum. Torymus Druparum, Boh. Kongl. Vet. Acad. Handl. 1833, 361. Callimome Arundinis, Curtis, Walker, Ent. Mag. i. 154.

a. England. Presented by F. Walker, Esq.

b. Sweden. Presented by F. Walker, Esq.

Callimome flavipes, Walker, Ent. Mag.i. 124. Cynips Urticæ? Perris. Ann. Soc. Ent. ix.

a. England. Presented by F. Walker, Esq.

b. Fontainbleau, France. Presented by the Entomological Club.

Callimome auratus. Torymus viridissimus, Boh. Kongl. Vet. Acad. Handl. 1833, 358. Callimome Dauci, Curtis, Walker, Ent. Mag. i. 124. Cynips auratus, Enc. Meth. Boyer de Fonscol. Ann. Sci. Nat. 1832, 284, 5. Torymus Muscarum.

Nees, Hym. Ich. aff. ii. 58.

a. England. Presented by F. Walker, Esq.

b. Sweden. Presented by F. Walker, Esq. 
Callimome basalis, Walker, Ent. Mag. i. 125.

a. England. Presented by F. Walker, Esq.

Callimome confinis, Walker, Ent. Mag. i. 125.

a. England. Presented by F. Walker, Esq.

Callimome inconspectus, Walker, Ent. Mag. i. 133.

a. England. Presented by F. Walker, Esq.

Callimome quercinus. Torymus quercinus, Boh. Kongl. Vet. Acad. Handl. 1833, 373. Callimome tarsalis, Walker, Ent. Mag. i. 134.

a. Sweden. Presented by F. Walker, Esq.

Callimome fastuosus. Torymus fastuosus, Boh. Kongl. Vet. Acad. Handl. 1833, 347. Callimome notatus, Walker, Ent. Mag. i. 134.

a. Sweden. Presented by F. Walker, Esq.

Callimome minutus, Walker, Ent. Mag. i. 137.

a. England. Presented by F. Walker, Esq.

b. Alten, Finmark. Presented by F. Walker, Esq.

Callimome Galii. Torymus Galii, Boh. Kong. Vet. Acad. Handl. 1833, 344. Callimome gracilis, Walker, Ent. Mag. i. 137.

a. Sweden. Presented by F. Walker, Esq.

Callimome chlorinus, Walker, Ent. Mag. i. 129.

a. England. Presented by F. Walker, Esq.

Callimome terminalis, Walker, Ent. Mag. i. 132.

a. England. Presented by F. Walker, Esq.

Callimome nigricornis. C. affinis, Ent. Mag. i. 133. Torymus caudatus, Boh. Kongl. Vet. Acad. Handl. 1833. Cinips affnis, Fonsc. Ann. Sci. Nat. xxvi. 283. Diplolepis nigricornis, Fab. Syst. Piezat. 150, 7. Ichneumon nigricornis, Fab. Ent. Syst. ii. 185, 216. Torymus admirabilis, Foerster, Monogr. Pterom. i. 32, 16, f. 2. T. caudatus, Nees ab Ess. Hym. Ich. aff. Mon. ii. 60. Diplolepis longicauda, Spinola, Ann. du Mus. vii. 148.

a. England. Presented by F. Walker, Esq.

b. Sweden. Presented by F. Walker, Esq.

Callimome saphirinus. Torymus saphirinus, Boh. Kongl. Vet. Acad. Handl. 1833. 371. Callimome littoralis, Walker, Ent. Mag. i. 134.

a. Sweden. Presented by F. Walker, Esq.

Callimome mutabilis, Walker, Ent. Mag. i. 127.

a. England. Presented by F. Walker, Esq.

Callimome microstigma, Walker, Ent. Mag. i. 127.

a. England. Presented by F. Walker, Esq. 
Callimome abbreviatus. Torymus abbreviatus, Boh. Kongl. Vet. Acad. Handl. 1833, 357. Callimome chloromerus, Walker, Ent. Mag. i. 128.

a. England. Presented by F. Walker, Esq.

b. Sweden. Presented by F. Walker, Esq.

c. Alten, Finmark. Presented by F. Walker, Esq.

Callimome latus, Walker, Ent. Mag. i. 128.

a. England. Presented by F. Walker Esq.

Callimome, bicolor, Walker, Ent. Mag. i. 130.

a. England. Presented by F. Walker, Esq.

Callimome euchlorus. Torymus euchlorus, Boh. Kongl. Vet. Acad. Handl. 1833, 359. Callimome æqualis, Walker, Ent. Mag. i. 129.

a. England. Presented by F. Walker, Esq.

b. Sweden. Presented by F. Walker, Esq.

Callimome curtus, Walker, Ent. Mag. i. 131.

a. England. Presented by F. Walker, Esq.

Callimome purpurascens. Torymus purpurascens, Boh. Kongl. Vet. Acad. Handl. 1833, 353. Callimome lætus, Walker, Ent. Mag. i. 136.

a. England. Presented by F. Walker, Esq.

b. Sweden. Presented by F. Walker, Esq.

Callimome parellinus. Torymus parellinus, Boh. Kongl. Vet. Acad. Handl. 1833, 372. Callimome posticus, Walker, Ent. Mag. i. 137.

a. England. Presented by F. Walker, Esq.

b. Sweden. Presented by F. Walker, Esq.

c. Hammerfest, Finmark. Presented by F. Walker, Esq.

Callimome exilis, Walker, Ent. Mag. i. 138.

a. England. Presented by F. Walker, Esq.

Caluimome pallidicornis. Torymus pallidicornis, Boh. Kongl. Vet. Acad. Handl. 1833, 363. Callimome nitidulus, Walker, Ent. Mag. i. 138.

a. England. Presented by F. Walker, Esq.

b. Sweden. Presented by F. Walker, Esq.

Callimome apicalis, Walker, Ent. Mag. i. 133.

a. England. Presented by F. Walker, Esq.

Callimome chrysocephalus. Torymus chrysocephalus, Boh. Kongl. Vet. Acad. Handl. 1833, 340. Callimome rudis, Walker, Ent. Mag. iv. 25.

a. England. Presented by F. Walker, Esq.

b. Sweden. Presented by F. Walker, Esq. 
Callimome nigritarsus, Walker, Ent. Mag. i. 135.

a. England. Presented by F. Walker, Esq.

Callimome inconstans, De Laporte, Ent. Mag. ii. 159. Torymus nigricornis, Boh. Kongl. Vet. Acad. Handl. 1833, 355. Evania Bedeguaris? Cuvier, Silberman, Rev. Ent. i. 154.

a. England. Presented by F. Walker, Esq.

b. France. Presented by F. Walker, Esq.

c. Sweden. Presented by F. Walker, Esq.

CaLlimome antennatus, Walker, Ent. Mag. i. 135.

a. England. Presented by F. Walker, Esq.

Callimone abdominalis, Walker, Ent. Mag. i. 126.

a. England. Presented by F. Walker, Esq.

Callimome leptocerus, Walker, Ent. Mag. i. 129.

a. England. Presented by F. Walker, Esq.

Callimome leucopterus, Walker, Ent. Mag. i. 130.

a. England. Presented by F. Walker, Esq.

Callimone autumnalis, Walker, Ent. Mag. i. 125. Cynips papaveris? Perris. Ann. Soc. Ent. ix.

a. England. Presented by F. Walker, Esq.

Callimome versicolor, Walker, Ent. Mag. i. 136.

a. England. Presented by F. Walker, Esq.

Callimome fuscicornis, Walker, Ent. Mag. i. 138.

a. England. Presented by F. Walker, Esq.

Callimome spilopterus. Torymus spilopterus, Boh. Kongl. Vet. Acad. Handl. 1833, 364.

a. Sweden. Presented by F. Walker, Esq.

Callimone cyaneus. Torymus cyaneus, Boh. Kongl. Vet. Acad. Handl. 1833, 366.

a. Sweden. Presented by F. Walker, Esq.

Calrimome cyaninus. Torymus cyaninus, Bohem. Kongl. Vet. Acad. Handl. 1833, 367.

a. Sweden. Presented by F. Walker, Esq.

Callimome Nonacris, Walker, Ann. Nat. Hist. x. 113.

a. Valparaiso, Chili. Presented by C. Darwin, Esq.

Callimome Eumelis, Walker, Ann. Nat. Hist. x. 114.

a. Valparaiso, Chili. Presented by C. Darwin, Esq.

Callimome Daonus, Walker, Ent. Mag. v. 474.

a. King George's Sound, Australia. Presented by C. Darwin, Esq.

Callinome Osinius, Walker, Mon. Chal. ii. 6.

a. King George's Sound, Australia. Presented by C. Darwin, Esq. 
Callimome Vibidia, Walker, Mon. Chal. ii. 7. a. Sydney, New South Wales. Presented by C. Darwin, Esq.

Callimome Caburus, Walker, Mon. Chal. ii. 63.

a. Bahia, Brazil. Presented by C. Darwin, Esq.

Callimome Sulcius, Walker, Mon. Chal. ii. 64.

a. Bahia, Brazil. Presented by C. Darwin, Esq.

Callimome splendidus, Barnstone, MSS. Walker, Ann. Nat. Hist. xiv. 14.

a. Martin's Falls, Albany River, Hudsur's Bay. Presented by G. Barnstone, Esq.

Callimome Cecidomyæ, Barnstone, MSS. Walker, Ann. Nat. Hist. xiv. 15.

a. Martin's Falls, Albany River, Hudson's Bay. Presented by G. Barnstone, Esq.

Callimome Theon, Walker, Ann. Soc. Ent. France, 2me Serie, i. 149.

a. St. John's Bluff, Florida. Presented by E. Doubleday, Esq.

Callimome Cissus, Walker, Ann. Soc. Ent. France, 2me Serie, i. 150 .

a. St. John's Bluff, Florida. Presented by E. Doubleday, Esq. Callimome Ea, Walker, Ann. Nat. Hist. xii. 104.

a. New York, North America. Presented by the Entomological Club.

7. Ormyrus, Westwood.

Ormyrus tubulosus, Ent. Mag. ii. 161. Cinips tubulosa, Fonsc. Ann. Sci. Nat. xxvi. 290, 18. Cyrtosoma Papaveris, Perris, Ann. Soc. Ent. France, ix.

$a, b$. France. Presented by the Entomological Club.

Ormyrus punctiger, Westwood, Phil. Mag. 3rd series, ii. 127. Entomologist, pl. O. fig. 1. Periglyphus gastris, Boh. Kongl. Vet. Acad. Handl. 1833, 379. Siphonura brevicauda, Nees. Hym.Ich. aff. Mon. ii. 83.

a. Finmark. Presented by F. Walker, Esq.

b. Sweden. Presented by F. Walker, Esq.

c. England. Presented by F. Walker, Esq.

Ormyrus Labotas, Walker, Ann. Soc. Ent. France, 2de Serie, i. 148.

a. St. John's Bluff, Florida. Presented by E. Doubleday, Esq. 
Fam. 5. Eucharida.

1. Eucharis, Latreille.

Euciaris Volusus, Walker, Mon. Chal. ii. 9. Entomologist, pl. P. fig. 1.

a. King George's Sound, Australia. Presented by C. Darwin, Esq.

Eucharis Fausta, Walker, Mon. Chal. ii. 10.

a. Hobart Town, Van Diemen's Land. Presented by C. Darwin, Esq.

Eucharis Valgius, Walker, Mon. Chal. ii. 11.

a. Sydney, New South Wales. Presented by C. Darwin, Esq.

Eucharis Theocles, Walker. Mon. Chal.ii. 11.

a. Sydney, New South Wales. Presented by C. Darwin, Esq.

Eucharis Iello, Walker, Mon. Chal. ii. 12. Entomologist, pl. P. fig. 3.

a. Hobart Town, Van Diemen's Land. Presented by C. Darwin, Esq.

Eucharis Zalates, Walker, Mon. Chal. ii. 13. Entomologist, pl. P. fig 4.

a. Sydney, New South Wales. Presented by C. Darwin, Esq.

b. King George's Sound, Australia. Presented by C. Darwin, Esq.

Eucharis Eribotes, Walker, Mon. Chal. ii. 14.

a. Sydney, New South Wales. Presented by C. Darwin, Esq.

b. Hobart Town, Van Diemen's Land. Presented by C. Darwin, Esq.

Eucharis Xeniades, Walker, Mon. Chal. ii. 15.

a. Sydney, New South Wales. Presented by C. Darwin, Esq.

Eucharis Rapo, Walker, Mon. Chal. ii. 66.

a. Bahia, Brazil. Presented by C. Darwin, Esq.

Eucharis adscendens, Latr. Hist. Nat. Crust. et Ins. xiii. 210.

Cynips adscendens, Fab. Mant. i. 251. Ent. Syst. ii. 100, 1.

Panz. Faun. Germ. fasc. 88, 18. Vill. Ent. Linn. iii. 77, 26. $a, b$. England.

Eucharis Eginetus. See Appendix.

a. Philippine Islands. From Mr. Wood's collection.

Eucharis Larymna. See Appendix.

a. Australia.

Eucharis Pyttalus. See Appendix.

a. Adelaide, Australia. Presented by the Entomological Club. 


\section{Thoracantha, Latreille.}

Thoracantha furcata. Eucharis flabellatus, mas, Fab. Syst. Piezat. 158. Eucharis furcata, fem. Fab. Syst. Piezat. 158. Entomologist, pl. P. fig. 2. a, b. Bahia, Brazil. Presented by C. Darwin, Esq. $c-e$. Sierra Leone, Africa. Presented by the Rev. D. F. Morgan. Thoracantha striata, Perty, Del. $t .28, f .15,16$. a. Minas Geraes, Brazil. From M. Parzudaki's collection.

Thoracantha Latreillii, Guerin, Icon. Règne An. Insectes, pl. 67. a. Bahia, Brazil. Presented by C. Darwin, Esq.

Thoracantha Nasua. See Appendix.

a. Philippine Islands. From Mr. Wood's collection.

\section{Schizaspidia, Westwood.}

Schizaspidia furcifer, Westw. Proc. Zool. Soc. 1835. 69. $a, b$. Bengal.

\section{Perilampus, Latreille.}

Perilanpus Entellus, Walker, Ann. Nat. Hist. xii. 103. $a-f$. Ohio, North America. Presented by the Entomological Club.

g. Georgia. From Mr. Abbot's collection.

Perilampus Alexinus. See Appendix.

a. Georgia. From Mr. Abbot's culleetion.

Perilampus violaceus, Latr. Gen. Crust. \&c. iv. 30. Cinipsillum violaceum, Lam. Anim. sans Vert. iv. 157. Cynips violacea, Latr. Hist. Nat. des Ins. xiii. 222, 3. Chalcis violacea, Panż. Faun. Ins. 88, 15. Jiplolepis violacea, Fab. Syst. Piezat. 149, 4. Perilampus pallipes, Curt. Brit. Ent. 158. Ent. Mag. i. 141. P. nigricornis, Newman, Ent. Mag. i. 141. P. ruficornis, Latr. Gen. \&c. iv. 30. Cinips ruficornis, Latr. Hist. Nat. \&c. xiii. 222, 2. Diplolepis ruficornis, Fab. Syst. Piez. 149, 1. Coquib. Ill. Icon. i. t. 1.f. 8.

a. England.

Perilampus Italicus, Latr. Ger. Crust. et Ins. iv. 30. Diplolepis Italica, Fab. Syst. Piezat. 149, 3. Cynips Italica, Fab. Ent. Syst.

a. England.

Perilampus lævifrons, Dalm. Kongl. Vet. Acad. Handl. 1822.

a. Sweden. Presented by F. Walker, Esq.

Perilampus Saleius, Walker, Mon. Chal. ii. 16.

a. King George's Sound, Australia. Presented by C. Darwin, Esq. 


\section{Cratomus, Dalman.}

Cratomus megacephalus, Dalm. Kongl. Vet. Acad. Handl. 1820. Caratomus megacephalus, Dalm. Kongl. Vet. Acad. Handl. 1822. Cynips megacephala, Fab. Ent. Syst. ii. 103, 17. Diplolepis megacephala, Fab. Syst. Piezat. 149, 21, 2. $a, b$. England.

Fam. 6. Agaonider.

1. Agaon, Dalman.

Agaon paradoxum, Dalm. Kongl. Vet. Acad. Handl. 1818. 69. t. xi. A.

a. Sierra Leone. Presented by the Rev. D. F. Morgan.

2. IDARNES, Walker.

Idarnes Carme, Walker, Ann. Nat. Hist. xii. 47.

$a$. St. Vincent's Island, West Indies. Presented by the Entomological Club.

\section{Fam. 7. Spalanginde.}

\section{Spalangia, Latr.}

Spalangia nigra, Latr. Hist. Nat. des Crust. et des Ins. xiii. 228.

S. Endius, Walker, Mon. Chal. ii. 96.

a. James' Isle, Galapagos. Presented by C. Darwin, Esq.

b. England. Presented by F. Walker, Esq.

c. Sweden. Presented hy F. Walker, Esq.

d. Jersey. Presented by the Entomological Club.

\section{Cerocephala, Westwood.}

Cerocephala formiciformis. Walker, Ent. Mag. ii. 149. Ento. mologist, pl. N. fig. 4. Theocolax formiciformis, Westwood, Lond. and Edin. Phil. Mag. Third Series, i. 127. Loesthia vespertina, Haliday, Ent. Mag. i. 335.

a. b. England. Presented by Lady Smirke.

c. France.

Fam. 8. Pteromalide.

1. Asaphis, Walker.

Asaphis ænea. A. vulgaris, Walker, Ent. Mag. ii. 152. Ann. Nat. Hist. x. 114. Eurytoma ænea, Nees. Hym. Ich. afj. Mon. ii. 42. 
a. England. Presented by F. Walker, Esq.

b. Finmark. Presented by F. Walker, Esq.

c. Valparaiso, Chili. Presented by C. Darwin, Esq.

\section{Macroglenes, Westwood.}

Macroglenes pénetrans, Ent. Mag. ii. 150. M. oculatus, Westwood, Lond. and Edin. Phil. Mag. Third Series, i. 127. Ichneumon penetrans, Kirby, Linn. Trans V.pl. 4 fig. 10. Stenophrus compressus, Foerster, Mon. Pteromal, -i. 40.

a. England. Presented by F. Walker, Esq.

b. Jersey. Presented by the Entomological Club.

Macroglenes umbellatarum, Haliday, Trans. Ent. Soc. 111, 295.

a. England. Presented by F. Walker, Esq.

Macroglenes microcerus, Haliday, Trans. Ent. Soc. 111, 295, Pirene graminea, Haliday, Ent. Mag. i. 338.

a. England. Presented by F. Walker, Esq.

\section{Calypso, Haliday.}

Calypso serratulæ, Haliday, Trans. Ent. Soc. 111, 296.

a. England. Presented by F. Walker, Esq.

\section{Pirene, Haliday.}

Prrene varicornis, Haliday, Ent. Mag. i. 337. Entomologist, pl. N. $f .3$.

a. England. Presented by F. Walker, Esq.

Pirene rubi, Haliday, Trans. Ent. Soc. 111, 296.

a. England. Presented by F. Walker, Esq.

Pirene chalybea, Haliday, Ent. Mag. i. 338. Corynocere brevicornis? Nees. ab. Ess. Hym. Ich. aff. ii. 124.

a. England. Presented by F. Walker, Esq.

Pirene eximia, Haliday, Ent. Mag. i. 338. Corynocere deplana? Nees. ab. Ess. Hym. Ich. aff. ii. 123.

a. England. Presented by F. Walker, Esq.

\section{Gastrancistrus, Westwood.}

Gastrancistrus fuscicurnis, Walker, Ent. Mag. xi. 171.

a. England. Presented by F. Walker, Esq.

Gastrancistrus compressus, Walker, Ent. Mag. xi. 172.

a. England. Presented by F. Walker, Esq. 
Gastrancistrus, tenuicornis, Walker, Ent. Mag. ii. 172.

a. England. Presented by F. Walker, Esq.

Gastrancistrus vagans, Westwood, Lond. and Edin. Phil. Mag. 3rd Series. ii. 444.

a. England. Presented by F. Walker, Esq.

Gastrancistrus atropurpureus, Walker, Ent. Mag. ii. 173.

a. England. Presented by F. Walker, Esq.

Gastrancistrus laticornis, Walker, Ent. Mag. ii. 174. Entomologist, p. D. f. 2.

a. England. Presented by F. Walker, Esq.

Gastrancistrus fumipennis, Walker, Ent. Mag. ii. 174.

a. England. Presented by F. Walker, Esq.

Gastrancistrus unicolor, Walker, Ent. Mag. ii. 175.

a. England. Presented by F. Walker, Esp.

Gastrancistrus vulgaris, Walker, Ent. Mag. ii. 175.

a. England. Presented by F. Walker, Esq.

Gastrancistrus terminalis, Walker, Ent. Mag. ii. 176.

a. England. Presented by F. Walker, Esq.

Gastrancistrus annulipes, Walker, Ent. Mag. ii. 176.

a. England. Presented by F. Walker, Esq.

Gastrancistrus crassus, Walker, Ent. Mag. ii. 177.

a. England. Presented by F. Walker, Esq.

Gastrancistrus angulus, Walker, Ent. Mag. ii. 177.

a. England. Presented by F. Walker, Esq.

Gastrancistrus acutus, Walker, Ent. Mag. ii. 177.

a. England. Presented by F. Walker, Esq.

Gastrancistrus Lœlianus, Walker. See Appendix.

a. England. Presented by F. Walker, Esq.

Gastrancistrus Polles, Walker, Ann. Nat. Hist. xi. 186.

a. Coquimbo, Chili. Presented by C. Darwin, Esq.

Gastrancistrus Cephalon, Walker, Ann. Nat. Hist. xi. 30.

a. Concepcion, Chili. Presented by C. Darwin, Esq.

Gastrancistrus? Menœtes. Miscogaster Menœtes, Walker,

Mon. Chal. ii. 20.

a. Sydney, New South Wales. Presented by C. Darwin, Esq.

Gastrancistrus autumnalis. Glyphe autumnalis, Walker, Ent.

Mag. ii. 171.

a. England. Presented by F. Walker, Esq. 


\section{Micradelus, Walker.}

Micradelus rotundus, Walker, Ent. Mag. ii. 170.

a. England. Presented by F. Walker, Esq.

7. URolepis, Walker.

Urolepis maritimus. Ormocerus maritimus, Walker, Ent. Mag. ii. 169. Miscogaster Stygne, Walker, Mon. Chal. i. 201.

a. England. Presented by F. Walker, Esq.

8. Ormocerus, Walker.

Ormocerus vernalis, Walker, Ent. Mag. ii. 169.

a. England. Presented by F. Walker, Esq.

Ormocerus latus, Walker, Ent. Mag. ii. 168.

a. England. Presented by F. Walker, Esq.

Ormocerus simplex, Walker, Ent. Mag. ii. 169.

a. England. Presented by F. Walker, Esq.

Ornocerus Trasullus, Walker, Mon. Chal. i. 207.

a. England. Presented by F. Walker, Esq.

\section{Cyrtogaster, Walker.}

Cyrtogaster vulgaris, Walker, Ent. Mag. i. 383. Chrysolampus tristis? Nees. Hym. Ich. aff. Mon. ii. 128.

a. England. Presented by F. Walker, Esq.

b. Finmark. Presented by F. Walker, Esq.

Cyrtogaster rufipes, Walker, Ent. Mag. i. 383.

a. England. Presented by F. Walker, Esq.

b. Finmark. Presented by F. Walker, Esq.

10. Pachylarthrus, Westwood.

Pachylarthrus smaragdinus. Walker, Ent. Mag. i. 457. Phagonia smaragdina, Curtis, Brit. Ent. 427.

a. England. Presented by F. Walker, Esq.

Pachylarthrus flavicornis. Walker, Ent. Mag. i. 457. Phagonia flavicornis, Haliday, MSS. Curt. Brit. Ent. 427.

a. England. Presented by F. Walker, Esq.

Pachylarthrus patellanus, Walker, Ent. Mag. i. 458. Diplolepis patellana, Dalm. Kongl. Vet. Acad. Handl. 18-80. Pachylarthrus insignis, Westwood, Phil. Mag. Third Series. ii. 127. Phacostomus patellanus, Nees. ab Ess. Hym. Ich. aff. Mon. ii. 121. 
a. England. Presented by F. Walker, Esq.

b. Finmark. Presented by F. Walker, Esq.

c. Jersey. Presented by the Entomological Club.

d. Clermont, France. Presented by the Entomological Club.

Pachylarthrus Sariaster, Walker, Ann. Nat, Hist. x. 271.

a. Valdivia, Chili. Presented by C. Darwin, Esq.

Pachylarthrus Cleodoxa, Walker, Ann. Nat. Hist. xi. 116.

a. Lima, Peru. Presented by C. Darwin, Esq.

Pachylarthrus Suilius. Miscogaster Suilius, Walker, Mon.

Chal. i. 202.

a. England. Presented by F. Walker, Esq.

Pachylarthrus Tyrrhæus. MiscogasterTyrrhæus, Walker, Mon .

Chal. i. 204.

a. England. Presented by F. Walker, Esq.

Pachylarthrus Eurybia. Miscogaster Eurybia, Walker, Mon.

Chal. i. 203.

a. England. Presented by F. Walker, Esq.

Pachylarthus Mustela, Walker, Mon. Chal. i. 194, 1.

a. Fontainbleau, France. Presented by F. Walker, Esq.

\section{Dicyclus, Walker.}

Dicyclus æneus, Walker, Ent. Mag. i. 456. Mon. Chal. i. 277. a. England. Presented by F. Walker, Esq.

Dicyclus fuscicornis, Walker. Ent Mag. i. 456. Mon. Chal. i. 277.

a. England. Presented by F. Walker, Esq.

Dicyclus Lynastes, Walker, Ann. Nat. Hist. x. 271.

a. Valdivia, Chili. Presented by C. Darwin, Esq.

Dicyclus Arduine, Walker, Ann. Nat. Hist. xi. 115.

a. Lima, Peru. Presented by C. Darwin, Esq.

Dicyclus tristis, Walker, Ent. Mag. i. 456. Mon. Chal. i. 277.

a. England. Presented by F. Walker, Esq.

Dicyclus circulus, Walker, Ent. Mag. i. 456. Mon. Chal. ii. 277.

a. England. Presented by F. Walker, Esq.

Dicyclus cinctipes. Miscogaster cinctipes, Walker, Ent. Mag. i. 462. Mon. Chal. i. 287.

a. England. Presented by F. Walker, Esq.

Dicyclus nigroæneus. Miscogaster nigroænea, Walker, Ent. Mag. i. 462. Mon. Chal. i. 288.

a. England. Presented by F. Walker, Esq. 
Dicyclus Deione. Miscogaster Deione, Walker, Mon. Chal. i. 199.

a. England. Presented by F. Walker, Esq.

b. Fontainbleau, France. Presented by the Entomological Club.

\section{Toxeuma, Walker.}

Toxeuma Ericæ, Walker, Ent. Mag. i. 379. The Entomologist, pl. D. fig. 1. T. fuscicornis Var. Ent. Mag. i. 462. M. lugubris Var. Ent. Mag. i. 462.

a. England. Presented by F. Walker, Esq.

13. Sphegigaster, Spinola.

Sphegigaster aculeata. Merismus aculeatus, Walker, Ent. Mag. i. 375.

a. England. Presented by F. Walker, Esq.

Sphegigaster fronto. Merismus fronto, Walker, Ent. Mag. i. 376 .

a. England. Presented by F. Walker, Esq.

Sphegigaster megapterus. Merismus megapterus, Walker, Ent. Mag. i. 377.

a. England. Presented by F. Walker, Esq.

Sphegigaster flavicornis. Merismus flavicornis, Walker, Ent.

Mag. i. 377. The Entomologist, pl. C. fig. 1. Sphegigaster pallicornis? Spinola. Ann. Mus. vii. 149. Diplolepis pallicornis? Spin. Ins. Lig. Fasc. 4, 227, 78. Chrysolampus pallicornis? Nees. Hym. Ich. aff, Mon. ii. 132.

a. England. Presented by F. Walker, Esq.

\section{Prosodes, Walker.}

Prosodes ater, Walker, Ent. Mag. i. 374. The Entomologist, pl. C. fig. 3, $a$.

a. England. Presented by F. Walker, Esq.

15. Syntomopus, Walker.

Syntomopus incurvus, Walker, Ent. Mag. i. 372.

a. England. Presented by F. Walker, Esq.

Syntomopus Cyzicus, Walker. Miscogaster Cyzicus, Mon.

Chal. i. 200, 7.

a. England. Presented by F. Walker, Esq. 
16. Megorismus, Walker.

Megorismus Daiphron. Miscogaster Daiphron, Walker, Mon. Chal. i. 198.

a. England. Presented by F. Walker, Esq.

17. Panstenon, Walker.

Panstenon Oxylus. Miscogaster, Oxylus, Walker, Mon. Chal. i. 196.

a. England. Presented hy F. Walker, Esq.

18. Paphagus, Walker.

Paphagus Sidero, Walker, Ann. Nat. Hist. xii. 48.

a. St. Vincent's Island, West Indies. Presented by the Eutomological Club.

\section{Coryna, Walker.}

Conyna clavata, Walker, Ent. Mag. i. 380. The Entomologist, pl. C. fig. 2. Chrysolampus suspensus, Nees. Hym. Ich. aff. Mon. ii. 127.

a. England. Presented by F. Walker, Esq.

20. Lamprotatus, Westwood.

Lamprotatus gibbus. Miscogaster gibba, Walker, Ent. Mag. i. 459. Mon. Chal. i. 278.

a. England. Presented by F. Walker, Esq.

Lamprotatus elegans. Miscogaster elegans, Walker. Ent. Mag. i. 459. Mon. Chal. i. 278.

a. England. Presented by F. Walker, Esq.

Lamprotatus rufipes. Miscogaster rufipes, Walker, Ent. Mag. i. 459. Mon. Chal. i. 278.

a. England. Presented by F. Walker, Esq.

Lamprotatus obscurus. Miscogaster obscura, Walker, Ent. Mag.

i. 459. Mon. Chal. i. 280.

a. England. Presented by F. Walker, Esq.

Lamprotatus maculatus. Miscogaster maculata, Walker, Ent. Mag. i. 459, Mon. Chal. i. 279.

a. England. Presented by F. Walker, Esq.

$b$, Alten, Finmark. Presented by F. Walker, Esq.

Lamprotatus notatus. Miscogaster notata, Walker, Ent. Mag. i. 459. Mon. Chal. i. 280.

a. England. Presented by F. Walker, Esa. 
Lamprotatus hortensis. Miscogaster hortensis, Curtis' Guide, Walker, Ent. Mag. i. 460. Mon. Chal. i. 282.

a. England. Presented by F. Walker, Esq.

Lamprotatus antennatus. Miscogaster antennata, Walker, Ent.

Mag. i. 460. Mon. Chal. i. 281.

a. England. Presented by F. Walker, Esq.

Lamprotatus lucidus. Miscogaster lucida, Walker, Ent. Mag. i. 460. Mon. Chal. i. 282.

a. England. Presented by F. Walker, Esq.

Lamprotatus fuscipes. Miscogaster fuscipes, Walker, Ent. Mag. i. 460. Mon. Chal. i. 281.

a. England. Presented by F. Walker, Esq.

Eamprotatus Babilus. See Appendix.

a. England. Presented by F. Walker, Esq.

Lamprotatus splendens, Westwood, Loudon's Mag. Nat. Hist. vi.

121. Miscogaster chrysochlora, Walker, Ent. Mag. i. 461.

Mon. Chal. i. 283.

a. England. Presented by F, Walker, Esq.

Lamprotatus chrysochlorus, Haliday. See Appendix.

a. England. Presented by F. Walker, Esq.

Lamprotatus annularis. Miscogaster annularis, Curtis' Guide,

Walker, Ent. Mag. i.461. Mon. Chal. i. 284.

a. England. Presented by F. Walker, Esq.

Lamprotatus annulipes, Curtis' Guide, Walker, Ent. Mag. i. 461. Mon. Chal. i. 285.

a. England. Presented by F. Walker, Esq.

Lamprotatus tumidus. Miscogaster tumida, Walker, Ent. Mag. i. 463. Mon. Chal. i. 288.

a. England. Presented by F. Walker, Esq.

Lamprotatus fuscipennis. Miscogaster fuscipennis, Walker,

Ent. Mag. i. 459. Mon. Chal. i. 280.

a. England. Presented by F. Walker, Esq.

Lamprotatus obscuripennis. Miscogaster obscuripennis, Walker, Ent. Mag. i. 460. Mon. Chal. i. 281.

a. England. Presented by F. Walker, Esq.

Lamprotatus viridis. Miscogaster viridis, Walker, Ent. Mag. i. 461. Mon. Chal. i. 284.

a. England. Presented by F. Walker, Esq.

Lamprotatus tarsalis. Miscogaster tarsalis, Walker, Ent. Mag. i. 461. Mon. Chal. i. 285.

a. England. Presented by F. Walker, Esq. 
Lamprotatus diffinis, Walker, Ent. Mag. i.460. Mon. Chal. i. 283.

a. England. Presented by F. Walker, Esq.

Lamprotatus dissimilis. Miscogaster dissimilis, Walker, Ent? Mag. i. 463. Mon. Chal. i. 289.

a. England. Presented by F. Walker, Esq.

Lamprotatus semiauratus. Miscogaster semiaurata, Walker, Ent. Mag. i. 463. Mon. Chal. i. 289.

a. England. Presented by F. Walker, Esq.

Lampkotatus philochortoides. Miscogaster philochortoides, Walker, Ent. Mag. i. 463. Mon. Chal. i. 290.

a. England. Presented by F. Walker, Esq.

Lamprotatus brevis. Miscogaster brevis, Walker, Ent. Mag. i. 464. Mon. Chal. i. 291.

a. England. Presented by F. Walker, Esq.

Lamprotatus linearis. Miscogaster linearis, Walker, Ent. Mag. i. 464. Mon. Chal.i. 291.

a. England. Presented by F. Walker, Esq.

Lamprotatus filicornis. Miscogaster filicornis, Walker, Ent. Mag. i. 464. Mon. Chal. i. 291.

a. England. Presented by F. Walker, Esq.

Lamprotatus femoratus. Miscogaster femorata, Walker, Ent. Mag. i. 464. Mon. Chal. i. 291.

a. England. Presented by F. Walker, Esq.

Lamprotatus æneus. Miscogaster ænea, Walker, Ent. Mag. i. 461. Mon. Chal. i. 285.

a. England. Presented by F. Walker, Esq.

Lamprotatus Scoticus. Miscogaster Scotica, Walker, Ent. Mag. i. 461. Mon. Chal. i. 285.

a. Scotland. Presented by F. Walker, Esq.

Lamprotatus Alcander, Walker, Ann. Nat. Hist. xi. 30.

a. Concepcion, Chili. Presented by C. Darwin, Esq̨.

Lamprotatus Tubero, Walker, Ann. Nat. Hist. xi. 185.

a. Coquimbo, Chili. Presented by C. Darwin, Esq.

Lamprotatus Nævolus, Walker, Ann. Nat. Hist. xi. 185.

a. Coquimbo, Chili. Presented by C. Darwin, Esq.

Lamprotatus Natta, Walker, Ann. Nat. Hist. x. 271.

a. Valdivia, Chili. Presented by C. Darwin, Esq.

Lamprotatus Bisaltes, Walker, Ann. Nat. Hist. x. 272.

a. Valdivia, Chili. Presented by C. Darwin, Esq. 
Lamprotatus Orobia, Walker, Ann. Nat. Hist. x. 272.

a. Valdivia, Chili. Presented by C. Darwin, Esq.

Lamprotatus Cœcina, Walker, Ann. Nat. Hist. x. 114.

a. Valparaiso, Chili. Presented by C. Darwin, Esq.

Lamprotatus Diœus, Walker, Ann. Nat. Hist. xiv. 16.

a. Martin's Falls, Albany River, Hudson's Bay. Presented by

G. Barnston, Esq.

Lamprotatus Habis, Walker, Ann. Soc. Ent. France, 2me. Série, i. 155.

a. St. John's Bluff, Florida. Presented by E. Doubleday, Esq.

Lamprotatus Salemus, Walker, Ann. Soc. Ent. France, $2 m e$.

Série. i. 156.

a. St. John's Bluff, Florida. Presented by E. Doubleday, Esq.

Lamprotatus Cyrnus, Walker, Ann. Soc. Ent. France, $2 m e$.

Serie i. 157.

a. St. John's Bluff, Florida. Presented by E. Doubleday, Esq.

Lam protatus Trypherus, Walker, Ann. Soc. Ent. France, $2 m e$. Serie i. 158.

a. St. John's Bluff, Florida. Presented by E. Doubleday, Esq.

Lamprotatus Numitus, Walker, Ann. Nat. Hist. xi. 184.

a. Isle of Chonos, Chili. Presented by C. Darwin, Esq.

Lamprotatus Ciron. Miscogaster Ciron, Walker, Mon. Chal. ii. 16.

a. Hobart Town, Van Dieman's Land. Presented by C. Darwin, Esq.

Lamprotatus Nelo. Micogaster Nelo, Walker, Mon. Chal. ii. 17.

a. King George's Sound, Australia. Presented by C. Darwin, Esq.

Lamprotatus Nicon. Miscogaster Nicon, Walker, Mon. Chal. ii. 17.

a. Sydney, New South Wales. Presented by C. Darwin, Esq.

Lamprotatus Thera. Miscogaster Thera, Walker, Mon. Chal. ii. 18.

a. Hobart Town, Van Diemen's Land. Presented by C. Darwin, Esq.

Lamprotatus Hecatœus. Miscogaster Hecatœus, Walker, Mon. Chal. ii. 19.

a. Hobart Town, Van Diemen's Land. Presented by C. Darwin, Esq.

Lamprotatus Myeon. Miscogaster Myeon, Walker, Mon. Chal. ii. 19.

a. Sydney, New South Wales. Presented by C. Darwin, Esq. 
Lamprotatus Damia. Miscogaster Damia, Walker, Mon. Chal. ii. 21.

a Sydney, New South Wales. Presented by C. Darwin, Esq.

Lamprotatus Bato. Miscogaster Bato, Walker, Mon. Chal. ii. 22.

a. Hobart Town, Van Diemen's Land. Presented by C. Darwin, Esq.

Lamprotatus Dioxippe. Miscogaster Dioxippe, Walker, Mon. Chal. ii. 67.

a. Bahia, Brazil. Presented by C. Darwin, Esq.

Lamprotatus Hages. Miscogáster Hages, Walker, Mon. Chal. ii. 83 .

a. Isle of Chiloe, Chili. Presented by C. Darwin, Esq.

Lamprotatus Eleus. Miscogaster Eleus, Walker, Mon. Chal. ii. 85.

a. Isle of Chiloe, Chili. Presented by C. Darwin, Esq.

21. Seladerma, Walker.

Seladerma lætum, Walker, Ent. Mag. ii. 289.

a. England. Presented by F. Walker, Esq.

Seladerma bicolor, Walker, Ent. Mag. ii. 289.

a. England. Presented by F. Walker, Esq.

Seladerua convexum, Walker, Ent. Mag. ii. 290.

a. England. Presented by F. Walker, Esq.

Se laderma Mazares, Walker, Ann. Nat. Hist. xiv. 337.

a. Alten, Finmark. Presented by F. Walker, Esq.

Seladerma Athanis. Miscogaster Athanis, Walker, Mon. Chal. ii. 26.

a. Sydney, New South Wales. Presented by C. Darwin, Esq.

b. King George's Sound, Australia. Presented by C. Darwin, Esq.

Seladerma Letus. Miscogaster Letus, Walker, Mon. Chal. ii. 26.

a. Hobart Town, Van Diemen's Land. Presented by C. Darwin, Esq.

Seladerma Cernus. Miscogaster Cernus, Walker, Mon. Chal. ii. 22.

a. Hobart Town, Van Dieman's Land. Presented by C. Darwin, Esq.

Seladerma Epulo, Walker, Mon. Chal. ii. 86. a. Isle of Chiloe, Chili. Presented by C. Darwin, Esqu. 


\section{Semiotus, Walker.}

Semiotus clarus, Walker, Ent. Mag. ii. 291. a. England. Presented by F. Walker, Esq.

Semiотus tarsalis, Walker, Ent. Mag. ii. 292.

a. England. Presented by F. Walker, Esq.

Semiotus varians, Walker, Ent. Mag. ii. 293. Entomologist, pl. I. $f .2$.

a. England. Presented by F. Walker, Esq.

b. Clermont, France. Presented by the Entomological Club.

Semiotus præstans, Walker, Ent. Mag. ii. 293.

a. England. Presented by F. Walker, Esq.

Semiotus diversus, Walker, Ent. Mag. ii. 294.

a. England. Presented by F. Walker, Esq.

b. Jersey. Presented by the Entomological Club.

Semiotus quadratus, Walker, Ent. Mag. ii. 295.

a. England. Presented by F. Walker, Esq.

Semiotus mœrens, Walker, Ent. Mag. ii. 295.

a. England. Presented by F. Walker, Esq.

Semiotus Dice. Miscogaster Dice, Walker, Mon. Chal. ii. 24.

b. King George's Sound, Australia. Presented by C. Darwin, Esq.

Semiotus Theope. Miscogaster Theope, Walker, Mon. Chal. ii. 25.

a. King George's Sound, Australia. Presented by C. Darwin, Esq.

Semiotus Merula. Miscogaster Merula, Walker, Mon. Chal. ii. 25.

a. Hobart Town, Van Diemen's Land. Presented by C. Darwin, Esq.

23. Systasis, Walker.

Systasis encyrtoides, Walker, Ent. Mag. ii. 296. Entomologist, pl. B. f. 1 .

a. England. Presented by F. Walker, Esq.

Systasis tenuicornis, Walker, Ent. Mag. ii. 297.

a. England. Presented by F. Walker, Esq.

\section{Isocyrtus, Walker.}

Isocyrtus lætus, Walker, Ent. Mag. i. 466. Mon. Chal. i. 292.

Miscogaster breviventris, Ent. Mag. i. 462.

a. England. Presented by F. Walker, Esq. 
Isocyrtus collaris. Pteromalus collaris, Walker, Ent. Mag. iii. 472 .

a. England. Presented by F. Walker, Esq.

\section{Pachyneuron, Walker.}

Pachyneuron formosum, Walker, Ent. Mag. i. 380.

a. England. Preseuted by F. Walker, Esq.

Pachyneuron Albutius, Walker, Ann. Soc. Ent. France, $2 m e$. Série i. 158.

a. St. John's Bluff, Florida. Presented by E. Doubleday, Esq.

\section{Micromelus, Walker.}

Micromelus rufomaculatus, Walker, Ent. Mag, i. 465.

a. England. Presented by F. Walker, Esq.

Micromelus pyrrhogaster, Haliday, MSS. Walker, Ent. Mag. i. 465 .

a. England. Presented by F. Walker, Esq.

Micromelus Silanus, Walker, Ann. Nat. Hist. xii. 46.

a. Mount Wellington, Hobart Town, Australia. Presented by C. Darwin, Esq.

Micromelus Cyrene, Walker, Ann. Soc. Ent. France, 2 me Série. i. 154.

a. St. John's Bluff, Florida. Presented by E. Doubleday, Esq.

\section{Meraporus, Walker.}

Meraporus graminicula, Walker, Ent. Mag. ii. 299.

a. England. Presented by F. Walker, Esq.

Meraporus alatus, Walker, Ent. Mag. ii. 300.

a. England. Presented by F. Walker, Esq.

28. Norbanus, Walker.

Norbanus Dysaules, Walker, Ann. Soc. Ent. France, 2 me Serie, i. 159.

a. St. John's Bluff, Florida. Presented by E. Doubleday, Esq. Norbanus Pisias, Walker, Ann. Soc. Ent. France, 2 me Série, i. 163.

a. St. John's Bluff, Florida. Presented by E. Doubleday, Esq. 
29. Psilocera, Walker.

Psilocera Deiphon, 'Walker, Ann. Soc. Ent. France, 2me Série, i. 161 .

a. St. John's Bluff, Florida. Presented by E. Doubleday, Esq. Psilocera verticillata. Pteromalus verticillatus, Foerster, Mon. Pteromal. i. 24, 175.

a. France.

\section{Pteromalus, Swederus.}

Pteromalus cincticornis. Platyterma cincticorne, Walker, Ent. Mag. ii. 306.

- a. England. Presented by F. Walker, Esq.

Pteromalus teliformis. Platyterma teliforme, Walker, Ent. Mag. xi. 305.

a. England. Presented by F. Walker Esq.

b. Fontainbleau, France. Presented by the Entomological Club. Pteromalus nobilis. Platyterma nobile, Walker, Ent. Mag. ii. 304.

a. England. Presented by F. Walker, Esq.

Pteromalus comptus. Platyterma comptum, Walker, Ent. Mag. ii. 341 .

a. England. Presented by F. Walker, Esq.

Pteromalus decorus. Platyterma decorum, Walker, Ent. Mag. ii. 342.

a. England. Presented by F. Walker, Esq.

Pteromalus prasinus. Platyterma prasinum, Walker, Ent. Mag. ii. 305.

a. England. Presented by F. Walker, Esq.

Pteromalus femoralis. Platyterma femorale, Walker, Ent. Mag. ii. 341 .

a. England. Presented by F. Walker, Esq.

Pteromalus Nephele. Platyterma Nephele. Walker, Ann. Nat. Hist. xi. 186.

a. Coquimbo, Chili. Presented by C. Darwin, Esq.

Preromalus fasciiventris. Mesopolobus fasciiventris, Westwood, Lond. and Edin. Phil. Mag. Third Series. ii. 12, 445. Eutelus fulvicornis, Walker, Ent. Mag. ii. 363. Pteromalus fasciculatus, Foerster, Mon. Pteromal. i. 11.

a. England Presented by F. Walker, Esq.

Preromalus tibialis. Platymesopus tibialis, Westwood, Lond. and Edin. Phil. Mag. Third Series, ii. 12, 444.

a. England Presented by F. Walker, Esq. 
Pteromalus dilectus. Eutelus dilectus, Walker, Ent. Mag. ii. 356. Amblymerus amœnus, Walker, Ent. Mag. ii. 307.

a. England. Presented by F. Walker, Esq.

Pteromalus validus. Amblymerus validus, Walker, Ent. Mag. ii. 308 .

a. Englaud. Presented by F. Walker, Esq.

Pteromalus ruralis. Amblymerus ruralis, Walker, Ent. Mag. ii. 343 .

a. England. Presented by F. Walker, Esq.

Pteromalus linearis Amblymerus linearis, Walker, Ent. Mag. ii. 349.

a. England. Presented by F. Walker, Esq.

Pteromalus temperatus. Amblymerus températus, Walker, Ent. Mag. ii. 349.

a. England. Presented by F. Walker, Esq.

Pteromalus placidus. Eutelus placidus, Walker, Ent. Mag. ii. 359 .

a. England. Presented by F. Walker, Esq.

Pteromalus jucundus. Eutelus jucundus, Walker, Ent. Mag. ii. 358 .

a. England. Presented by F. Walker, Esq.

Pteromalus platycerus. Eutelus platycerus, Walker, Ent. Mag. ii. 360 .

a. England. Presented by F. Walker, Esq.

Pteromalus sobrinus. Eutelus sobrinus, Walker, Ent. Mag. ii. 362 .

a. England. Presented by F. Walker, Esq.

Pteromalus stenomerus. Amblymerus stenomerus, Walker, Ent. Mag. ii. 350.

a. England. Presented by F. Walker, Esq.

Pteromalus stupidus. Amblymerus stupidus, Walker, Ent. Mag. ii. 348.

a. England. Presented by F. Walker, Esq.

Pteromalús tenuicornis. Amblymerus tenuicornis, Walker, Ent. Mag. ii. 347.

a. England. Presented by F. Walker, Esq.

Pteromalus pusillus. Amblymerus pusillus, Walker, Ent. Mag. ii. 347 .

a. England. Presented by F. Walker, Esq.

Pteromalus albitarsus. Amblymerus albitarsus, Walker, Ent. Mag. ii. 346.

a. England. Presented by F. Walker, Esq. 
Pteromaius fuscipes. Amblymerus fuscipes, Walker, Ent. Mag. ii. 346.

a. England. Presented by F. Walker, Esq.

Pteromalus modestus. Amblymerus modestus, Walker, Ent. Mag. ii. 344.

a. England. Presented by F. Walker, Esq.

Pteromalus truncatellus. Amblymerus truncatellus, Walker, Ent. Mag. ii. 344.

a. England. Presented by F. Walker, Esq.

Pteromalus Enicus. Walker, MSS. Amblymerus latus, Walker, Ent. Mag. ii. 343.

a. England. Presented by F. Walker, Esq.

Pteromalus campestris. Amblymerus campestris, Walker, Ent. Mag. ii. 343.

a. England. Presented by F. Walker, Esq.

Pteromalus dubius. Amblymerus dubius, Walker, Ent. Mag. ii. 308 .

a. England. Presented by F. Walker, Esq.

Pteromalus eximius. Eutelus eximius, Walker, Ent. Mag. ii. 360 .

a. England. Presented by F. Walker, Esq.

b. Clermont, France. Presented by the Entomological Club.

c. South of France. Presented by the Entomological Club.

Pteromalus fuscipennis. Eutelus fuscipennis, Walker, Ent. Mag. ii. 368.

a. England. Presented by F. Walker, Esq.

Pteromalus chlorospilus. Eutelus chlorospilus, Walker, Ent. Mag. ii. 368.

a. England. Presented by F. Walker, Esq.

Pteromalus semotus. Eutelus semotus, Walker, Ent. Mag. ii. 367 .

a. England. Presented by F. Walker, Esq.

Pteromalus altus. Eutelus altus, Walker, Ent. Mag. ii. 367.

a. England. Presented by F. Walker, Esq.

Pteromalus intermedius. Eutelus intermedius, Walker, Ent. Mag. ii. 366.

a. England. Presented by F. Walker, Esq.

Pteromalus elevatus. Eutelus elevatus, Walker, Ent. Mag. ii. 366 .

a. England. Presented by F. Walker, Esq.

Pteromalus posticus. Eutelus posticus, Walker, Ent. Mag. ii. 366 .

a. England. Presented by F. Walker, Esq. . 
Pteronalus gracilis. Eutelus gracilis, Walker, Ent. Mag. ii. 365 .

a. England. Presented by F. Walker, Esq.

Pteromalus planus. Eutelus planus, Walker, Ent. Mag. ii. 356.

a. England. Presented by F. Walker, Esq.

Pteromalus æquus. Eutelus æquus, Walker, Ent. Mag. ii. 364 .

a. England. Presented by F. Walker, Esq.

Pteronalus flavipes. Eutelus flavipes, Walker, Ent. Mag. ii. 364 .

a. England. Presented by F. Walker, Esq.

$b$. Clermont, France. Presented by the Entomological Club.

Pteromalus bicolor. Eutelus bicolor, Walker, Ent. Mag. ii. 361.

a. England. Presented by F. Walker, Esq.

Pteromalus inornatus. Eutelus inornatus, Walker, Ent. Mag. ii. 363.

a. England. Presented by F. Walker, Esq.

b. Alten, Finmark. Presented by F. Walker, Esq.

Pteromalus platynotus. Eutelus platynotus, Walker, Ent. Mag. ii. 361 .

a. England. Presented by F. Walker, Esq.

Pteromalus diffinis. Eutelus diffinis, Walker, Ent. Mag. ii. 358.

a. England. Presented by F. Walker, Esq.

Pteronalus signatus. Eutelus signatus, Walker, Ent. Mag. ii. 357.

a. England. Presented by F. Walker, Esq.

Pteronalus immaculatus. Eutelus immaculatus, Walker, Ent.

Mag. ii. 357. The Entomologist, pl. B. f. 4.

a. England. Presented by F. Walker, Esq.

Pteromalus cavus, Walker, Ent. Mag. ii. 477.

a. England. Presented by F. Walker, Esq.

$b$. South of France. Presented by the Entomological Club.

Pteromalus decedens, Walker, Ent. Mag. ii. 478.

a. England. Presented by F. Walker, Esq.

Pteromalus patulus, Walker, Ent. Mag. ii. 479. $a, b$. England. Presented by F. Walker, Esq.

Pteromalus amplus, Walker, Ent. Mag. ii. 480.

a. England. Presented by F. Walker, Esq. 
Pteromalus catillus, Walker, Ent. Mag. ii. 480. a. England. Presented by F. Walker, Esq.

Pteromalus deplanatus, Nees d'Essenbeck, Hym. Ich. aff. ii. 110. Pteromalus domesticus, Walker, Ent. Mag. ii. 481. Ann. Nat. Hist. xiv. 182.

a. England. Presented by F. Walker, Esq.

Pteromalus sylvicola, Walker, Ent. Mag. ii. 481.

a. England. Presented by F. Walker, Esq.

b. Alten, Finmark. Presented by F. Walker, Esq.

Pteromalus discus, Walker, Ent. Mag. ii. 482.

a. England. Presented by F. Walker, Esq.

Pteromalus Endomychi, Curtis, MSS. Ent. Mag. iii, 496.

a. England. Presented by F. Walker, Esq.

Pteromalus gynetelus, Walker, Ent. Mag. ii, 483.

a. England. Presented by F. Walker, Esq.

Pteromalus bracteatus, Walker, Ent. Mag. ii. 483.

a. England. Presented by F. Walker, Esq.

Pteromalus herbidus, Walker, Ent. Mag. ii. 484.

a. England. Presented by F. Walker, Esq.

Pteromalus lucidus, Walker, Ent. Mag. ii. 484.

a. England. Presented by F. Walker, Esq.

Pteromalus aspilus, Walker, Ent. Mag. ii. 485.

a. England. Presented by F. Walker, Esq.

Pteromalus flammiger, Walker, Ent. Mag. ii. 485.

a. England. Presented by F. Walker, Esq.

Pteromalus conspersus, Walker, Ent. Mag. ii.

a. England. Presented by F. Walker, Esq.

Pteromalus oxygene, Walker, Ent. Mag. ii. 486.

a. England. Presented by F. Walker, Esq.

Pteromalus grandis, Walker, Ent. Mag. ii. 487.

a. England. Presented by F. Walker, Esq.

Pтеromalus robustus, Walker, Ent. Mag. ii. 488.

a. England. Presented by F. Walker, Esq.

Pteromalus nubilus, Walker, Ent. Mag. ii. 488.

a. England. Presented by F. Walker, Esq.

Pteromalus perfectus, Walker, Ent. Mag. ii. 488.

a. England. Presented by F. Walker, Esq.

Pteromalus dives, Walker, Ent. Mag. ii. 489.

a. England. Presented by F. Walker, Esq.

Pteromalus obtusus, Walker, Ent. Mag. ii. 490.

a. England. Presented by F. Walker, Esq. 
Pteromalus chalceus, Walker, Ent. Mag. ii. 491.

a. England. Presented by F. Walker, Esq.

Pтeromalus brevicornis, Walker, Ent. Mag. ii. 491.

a. England. Presented by F. Walker, Esq.

Pteromalos affinis, Walker, Ent. Mag. ii. 492.

a. England. Presented by F. Walker, Esq.

Pteromalus fumipennis, Walker, Ent. Mag. ii. 492.

a. England. Presented by F. Walker, Esq.

Pte romalus redactus, Walker, Ent. Mag. ii. 492.

a. England. Presented by F. Walker, Esq.

Pteromalus epistenus, Walker, Ent. Mag. ii. 493.

a. England. Presented by F. Walker, Esq.

Pteromalus semifascia, Walker, Ent. Mag. ii. 494.

a. England. Presented by. F. Walker, Esq.

Pteromalus venustus, Walker, Ent. Mag. ii. 494.

a. England. Presented by F. Walker, Esq.

Pteromalus varius, Walker, Ent. Mag. ii. 495.

a. England. Presented by F. Walker, Esq.

Pteromalus sequester, Walker, Ent. Mag. ii. 495.

a. England. Presented by F. Walker, Esq.

Pteromalus decorus, Walker, Ent. Mag. ii. 496.

a. England. Presented by F. Walker, Esq.

Pteromalus famulus, Walker, Ent. Mag. ii. 496.

a. England. Presented by F. Walker, Esq.

Pтeromalus perpetuus, Walker, Ent. Mag. ii. 497. Pt. communis. Nees. Hym. Ich. aff. Mon. ii. 103 ?

a. England. Presented by F. Walker, Esq.

b. France. Presented by M. Brebisson.

Pteromalus viridulus, Walker, Ent. Mag. ii. 497.

a. England. Presented by F. Walker, Esq.

Pteromalus tenuis, Walker, Ent. Mag. ii. 498. Mon. Chai. i. 236.

a. England. Presented by F. Walker, Esq.

Pteromalus inops, Walker, Ent. Mag. ii. 499.

a. England. Presented by F. Walker, Esq.

Pteromalus inscitus, Walker, Ent. Mag. ii. 499.

a. England. Presented by F. Walker, Esq.

Pteromalus tristis, Walker, Ent. Mag. ii. 499.

a. England. Presented by F. Walker, Esq. 
Pteromalus microcerus, Walker, Ent. Mag. ii. 500.

a. England. Presented by F. Walker, Esq.

Pteromalus repandus, Walker, Ent. Mag. ii. 501.

a. England. Presented by F. Walker, Esq.

Pteromalus quadrinota, Walker, Ent. Mag. ii. 501.

a. England. Presented by F. Walker, Esq.

Pteromalus longicornis, Walker, Ent. Mag. iii. 94.

a. England. Presented by F. Walker, Esq.

Pteromalus subniger, Walker, Ent. Mag. iii. 95. Pt. caligatus,

Var, Walker, Ent. Mag. iii. 494.

$a, b$. England. Presented by F. Walker, Esq.

Pteromalus imbutus, Walker, Ent. Mag. iii. 96.

a. England. Presented by F. Walker, Esq.

Pteromalus junceus, Walker, Ent. Mag. iii. 182.

a. England. Presented by F. Walker, Esq.

Pteromalus spicatus, Walker, Ent. Mag. iii. 97.

a. England. Presented by F. Walker, Esq.

Pteromalus filicornis, Walker, Ent. Mag. iii. 183.

a. England. Presented by F. Walker, Esq.

Pteromalus muscarum, Walker, Ent. Mag. iii. 185. Ichneumon muscarum, Linn. Syst. Nat. ii. 938, 62. Faun. Suec. 1636. Cleptes muscarum, Fab. Syst. Peizat. 156-7. Cynips viridis, nitens, \&c. Geoff. Ins. ii. 308, 31.

a. England. Presented by F. Walker, Esq.

Pteromalus placidus, Walker, Ent. Mag. iii. $18 \%$.

a. England. Presented by F. Walker, Esq.

Pteromalus ovatus, Walker, Ent. Mag. iii. 187.

a. England. Presented by F. Walker, Esq.

Pteromalus contractus, Walker, Ent. Mag. iii. 188.

a. England. Presented by F. Walker, Esq.

Pteromalus linearis. Walker, Ent. Mag. iii. 189.

a. England. Presented hy F. Walker, Esq.

Preromalus formosus, Walker, Ent. Mag. iii. 189.

a. England. Presented by F. Walker, Esq.

Pteromalus fulviventris, Walker, Ent. Mag. iii. 190.

a. England. Presented by F. Walker, Esq.

Preromalus tricolor, Walker, Ent. Mag. iii. 190.

a.-z. England. Presented by F. Walker, Esq.

Pteromalus maculipennis, Walker, Ent. Mag. iii. 191.

a. England. Presented by F. Walker, Esq. 
Pteromalus congruus, Walker, Ent. Mag. iii. 194.

a. England. Presented by F. Walker, Esq.

Pteromalus nubilipennis, Walker, Ent. Mag. iii. 195.

a. England. Presented by F. Walker, Esq.

$b$. South of France. Presented by the Entomological Club.

Pteromalus rufiventris, Walker, Ent. Mag. iii. 192.

a. England. Presented by F. Walker, Esq.

Pteromalus transiens, Walker, Ent. Mag. iii. 192.

a. England. Presented by F. Walker, Esq.

Pteromalus apicalis, Walker, Ent. Mag. iii. 196.

a. England. Presented by F. Walker, Esq.

Ptfromalus hemipterus, Walker, Ent. Mag.

$a, b$. England. Presented by F. Walker, Esq.

Pteromalus cingulipes, Walker, Ent. Mag. iii. 197.

a. England. Presented by F. Walker, Esq.

Pteromalus albipennis, Walker, Ent. Mag. iii. 198.

a. England. Presented by F. Walker, Esq.

Pteromalus cupreus, Walleer, Ent. Mag, iii. 200.

a. England. Presented by F. Walker, Esq.

Pteromalus puparum, Swederus Kong. Vet. Acad. Handl. 1795.

Ichneumon puparum, Linn. Syst. Nat. ii. 939, 66. Diplolepis puparum, Fab. Syst. Piezat. 151, 15. Cynips puparum, Fourc. Ent. Par. ii. 387, 24. Ich. Antiope, Scop. Ent. Carn.

765. Cynips viridisericeus, \&c. Geoff. Ins. ii. 305. 24.

a. England. Presented by F. Walker, Esq.

$b$. France. Presented by M. Brebisson.

c. Italy. Presented by Dr. Leach.

P'te romalus mesochlorus, Walker, Ent. Mag. iii. 201.

a. England. Presented by F. Walker, Esq.

Preromalus lugubris, Walker, Ent. Mag. iii. 205.

a. England. Presented by F. Walker, Esq,

Pte romalus micans, Olivier, Mem. Soc. d'Agriculture, xvi. $47 \%$.

p. 3.f. 12. Pteromalus bellus, Walker, Ent. Mag. iii. 466.

a. England. Presented by F. Walker, Esq.

b. France. Presented by M. Brebisson.

Pteromalus chluris, Walker, Ent. Mag. iii. 467.

a. England. Presented by F. Walker, Esq.

Pteromalus constans, Walker, Ent. Mag. iii. 468.

a. England. Presented by F. Walker, Esq.

Pteromalus impar, Walker, Ent. Mag. iii. 469.

a. England. Presented by F. Walker, Esq. 
Pteromalus brevivitta, Walker, Ent. Mag. iii. 470. a. England. Presented by F. Walker, Esq. Pteromalus pulchripes, Walker, Ent. Mag. iii. 470. a. England. Presented by F. Walker, Esq.

Pteromalus continuus, Walker, Ent. Mag. iii. 471. a. England. Presented by F. Walker, Esq. Pteromalus Iera, Walker, Ann. Nat. Hist. xiv. 339. a. Alten, Finmark Presented by F. Walker, Esq. Preromalus Pyttalus, Walker, Ann. Nat. Hist. xiv. 340. a. Alten, Finmark. Presented by F. Walker, Esq.

Pteromalus nanus, Walker, Ent. Mag. iii. 472.

a. England. Presented by F. Walker, Esq.

Pтeromalus discolor, Walker, Ent. Mag. iii. 473.

a. England. Presented by F. Walker, Esq.

Pteromalus gaudens, Walker, Ent. Mag. iii. 473.

a. England. Presented by F. Walker, Esq.

Pteromalus mœrens, Walker, Ent.Mag. iii. 474.

a. England. Presented by F. Walker, Esq.

Pteromalus laticornis, Walker, Ent. Mag. iii. 475.

a. England. Presented by F. Walker, Esq.

Pteromalus chalcomelas, Walker, Ent. Mag. iii. 476.

a. England. Presented by F. Walker, Esq.

Pteromalus terminalis, Walker, Ent. Mag. iii. 476.

a. England. Presented by F. Walker, Esq.

Pteromalus compressus, Walker, Ent. Mag. iii. 477.

a. England. Presented by F. Walker, Esq.

Pteromalus attenuatus, Walker, Ent. Mag. iii. 479.

a. England. Presented by F. Walker, Esq.

Pteromalus mundus, Walker, Ent. Mag. iii. 479.

a. England. Presented by F. Walker, Esq.

Pteromalus amplus, Walker, Ent. Mag. iii. 480.

a. England. Presented by F. Walker, Esq.

Pteromalus divisus, Walker. Ent. Mag. iii. 480.

$a, b$. England. Presented by F. Walker, Esq.

Pteromalus cephalotes, Walker, Ent. Mag. iii. 481.

a. England. Presented by F. Walker, Esq.

Pteromalus eupterus, Walker, Ent. Mag. iii. 482.

a. England. Presented by F. Walker, Esq.

Pteromalus conifer, Walker, Ent. Mag. iii. 484.

a. England. Presented by F. Walker, Esq. 
Pte romalus sobrius Walker, Ent. Mag. iii. 484.

a. England, Presented by F. Walker, Esq.

Pteromalus fuscicornis, Walker, Ent. Mag. iii. 484.

a. England. Presented by F. Walker, Esq.

Pteromalus bifrons, Walker, Ent. Mag. iii. 485.

a. England. Presented by F. Walker, Esq.

Pteromalus epimelas, Walker, Ent. Mag. iii. 486.

a. England. Presented by F. Walker, Esq.

Pteromalus stenotelus, Walker, Ent. Mag. iii. 487.

a. England. Presented by F. Walker, Esq.

Pteromalus concisus, Walker, Ent. Mag. iii. 488.

a. England. Presented by F. Walker, Esq.

Pteromalus balux, Walker, Ent. Mag. iii. 418.

a. England. Presented by F. Walker, Esq.

Pteromalus hilaris, Walker, Ent. Mag. iii. 489.

a. England. Presented by F. Walker, Esq.

Preromalus orbiculatus, Walker, Ent. Mag. iii. 490.

a. England. Presented by F. Walker, Esq.

Pteromalus fulvipes, Walker, Ent. Mag. iii. 490.

a. England. Presented by F. Walker, Esq.

Pteromalus chrysos, Walker, Ent. Mag. iii. 491.

a. England. Presented by F. Walker, Esq.

Preromalus vitripennis, Walker, Ent. Mag. iii. 492.

a. England. Presented by F. Walker, Esq.

Pteromalus obscuratus, Walker, Ent. Mag. iii. 493.

a. England. Presented by F. Walker, Esq.

Pteromalus inclusus, Walker, Ent. Mag. iii. 493.

a. England. Presented by F. Walker, Esq.

Pteroma lus conterminus, Walker, Ent. Mag. iii. 494.

a. England. Presented by F. Walker, Esq.

Preromalus Ariomedes, Walker, Mon. Chal. i. 210.

a. England. Presented by F. Walker, Esq.

Pteromalus Cabarnos, Walker, Mon. Chal. i. 211.

a. England. Presented by F. Walker, Esq.

Pteromalus Orsetes, Walker, Mon. Chal, i. 211.

a. England. Presented by F. Walker, Esq.

Pteromalus Elymus, Walker, Mon. Chal. i. 212.

a. England. Presented by F. Walker, Esq.

Pteromalus Deucetius, Walker, Mon. Chal. i. 245.

a. England. Presented by F. Walker, Esq. 
Prenomalus Codrus, Walker, Mon. Chal. i. 249. a. England. Presented by F. Walker, Esq.

Preromalus Crotus, Walker, Mon. Chal. i. 252.

a. England. Presented by F. Walker, Esq.

Pteromalus Daimenes, Walker, Mon. Chal. i. 250.

a. England. Presented by F. Walker, Esq.

Pteromalus Mutia, Walker, Mon. Chal. i. 246.

a. England. Presented by F. Walker, Esq.

Pteromalus Vindalius, Walker, Mon. Chal. i. 256.

a. England. Presented by F. Walker, Esq.

Pteromalus Emathion, Walker, Mon. Chal.i. 243.

a. England. Presented by F. Walker, Esq.

Pteromalus Laogore, Walker, Mon. Chal. i. 267.

a. England. Presented by F. Walker, Esq.

Pteromalus Lentulus, Walker, Mon. Chal. i. 232.

a. England. Presented by F. Walker, Esq.

Pteromalus Mycale, Walker, Mon. Chal. i. 253.

a. England. Presented by F. Walker, Esq.

Pteromalus Cerpheres, Walker, Mon, Chal. i. 255.

a. England. Presented by F. Walker, Esq.

Pteromalus Ergias, Walker, Mon. Chal. i. 238.

a. England. Presented by F. Walker, Esq.

Pteromalus Janira, Walker, Mon. Chal. i. 248.

a. England. Presented by F. Walker, Esq.

Pтeromalus Ortalus, Walker, Mon. Chal. i. 241.

a. England. Presented by F. Walker, Esq.

Pteromalus Toxicrate, Walker, Mon. Chal. i. 226.

a. England. Presented by F. Walker, Esq.

Pteromalus Deiochus, Walker, Mon. Chal. i. 240.

a. England. Presented by F. Walker, Esq.

Pteromalus Hedymeles, Walker, Mon. Chal. i. 215.

a. England. Presented by F. Walker, Esq.

Pteromalus Zipætes, Walker, Mon. Chal. i. 213.

a. England. Presented by F. Walker, Esq.

Pteromalus Ceropasades, Walker, Mon. Chal. i. 214.

a. England. Presented by F. Walker, Esq.

Pteromalus Charops, Walker, Mon. Chal. i. 242.

a. England. Presented by F. Walker, Esq.

Pteromalus Cosingas, Walker, Mon. Chal. i. 263.

a. England. Presented by F. Walker, Esq. 
Pteromalus Alcman, Walker, Mon. Chal. i. 265.

a. England. Presented by F. Walker, Esq.

Pteromalus Artembares, Walker, Mon. Chal. i. 257.

a. England. Presented by F. Walker, Esq.

Pteromalus Apharetus, Walker. Mon. Chal.i. 228.

a. Eugland. Presented by F. Walker, Esq.

Preromalus Cabades, Walker, Mon. Chal. i. 264.

a. England. Presented by F. Walker, Esq.

Pte romalus Isarchus, Walker, Mon. Chal. i. 216.

a. England. Presented by F. Walker, Esq.

Pteromalus Thessalus, Walker, Mon. Chal. i. 268.

a. England. Presented by F. Walker, Esq.

Pteromalus Artemon, Walker, Mon. Chal. i. 218. $a, b$. England. Presented by F. Walker, Esq.

Pteromalus Pirus, Walker, Mon. Chal. i. 219. $a, b$. England. Presented by F. Walker, Esq.

Pteromalus Sisenna, Walker. Mon. Chal. i. 227.

a. England. Presented by F. Walker, Esq.

Pteromalus Lucilla, Walker, Mon. Chal. i. 231.

a. England. Presented by F. Walker, Esq.

Preromalus Crocale, Walker, Mon. Chal. i. 239.

a. England. Presented by F. Walker, Esq.

b. Alten, Finmark. Presented by F. Walker, Esq.

Pteronalus Telon, Walker, Mon. Chal. i. 216.

a. England. Presented by F. Walker, Esq.

Pteronalus Merope, Walker, Mon. Chal. i. 219.

a. England. Presented by F. Walker, Esq.

Preromalus Pione, Walker, Mon. Chal. i. 224.

a. England. Presented by F. Walker, Esq.

Pteromalus Orthia, Walker, Mon. Chal. i. 223.

a. England. Presented by F. Walker, Esq.

Pteromalus Saptine, Walker, Mon. Chal. i. 224.

a. England. Presented by F. Walker, Esq.

Pteromalus Deudorix, Walker, Mon. Chal. i. 225.

a. England. Presented by F. Walker, Esq.

Pteromalus Zonaras, Walker, Mon. Chal. i. 227.

a. England. Presented by F. Walker, Esq.

$b$. South of France. Presented by the Entomological Club.

Pteromalus Ornytus, Walker, Mon. Chal. i. 238.

a. England. Presented by F. Walker, Esq. 
Preromalus Leodocus, Walker, Mon. Chal. i. 237.

a. England. Presented by F. Walker, Esq.

Pteromalus Amphimedon, Walker, Mon. Chal.i. 235.

a. England. Presented by F. Walker, Esq.

Pteroma lus Irus, Walker, Mon. Chal. i. 235.

a. England. Presented by F. Walker, Esq.

Pteromalus Sunides, Walker. See Appendix.

a. England. Presented hy F. Walker, Esq.

Preromalus Odites, Walker. See Appendix.

a. England. Presented by F. Walker, Esq.

Pteromalus Leogoras, Walker, Mon. Chal. i. 269.

a. England. Presented by F. Walker, Esq.

Pteromalus Nestocles, Walker. See Appendix.

a. England. Presented by F. Walker, Esq.

Pteromalus Amyntor. See Appendix.

a. England. Presented by F. Walker, Esq.

Pteromalus Cercides, Walker. See Appendix.

a. England. Presented by F. Walker, Esq.

Pteromalus Bebryce, Walker, Mon. Chal. i. 273.

a. South of France. Presented by the Entomological Club.

Pteromalus Nypsius, Walker, Mon. Chal. i. 274.

a. South of France. Presented by the Entomological Club.

Pteromalus Vitula, Walker, Ann. Nat. Hist. xi. 187.

a. Coquimbo, Chili. Presented by C. Darwin, Esq.

Pteromalus Ornoe, Walker, Ann. Nat. Hist. xi. 187.

a. Coquimbo, Chili. Presented by C. Darwin, Esq.

Pteromalus Megareus, Walker, Ann. Nat. Hist.x. 272.

a. Valdivia, Chili. Presented by C. Darwin, Esq.

Pteromalus Gryneus, Walker, Ann. Nat. Hist. x. 115.

a. Valparaiso, Chili. Presented by C. Darwin, Esq.

Preromalus Archia, Walker, Ann. Nat. Hist. xi. 116.

a. Lima, Peru. Presented by C. Darwin, Esq.

Preromalus Unca, Walker, Mon. Chal. ii. 28.

a. Hobart Town, Van Dieman's Land. Presented by C. Darwin, Esq.

Pteromalus Niphe, Walker, Mon. Chal. ii. 20.

a. Hobart Town, Van Dieman's Land. Presented by C. Darwin, Esq.

Pteromalus Thestor, Walker, Mon. Chal. ii. 29.

a. Hobart Town, Van Dieman's Land. Presented by C. Darwin, Esq. 
Pteronalus Oceia, Walker, Mon. Chal. ii. 30.

a. Hobart Town, Van Diemen's Land. Presented by C. Darwin, Esq.

Pteromalus Europs, Walker, Mon. Chal. ii. 30.

a. Sydney, New South Wales. Presented by C. Darwin, Esq.

Pteromalus Euctemon, Walker, Mon. Chal. ii. 31.

a. Sydney, New South Wales. Presented by C. Darwin, Esq.

Pteromalus Bebius, Walker, Mon. Chal. ii. 31.

a. Sydney, New South Wales. Presented by C. Darwin, Esq.

Pteromalus Baton, Walker, Mon. Chal. ii. 32.

a. Hobart Town, Van Diemen's Land. Presented by C. Darwin, Esq.

b. Sydney, New South Wales. Presented by C. Darwin, Esq.

Pteromalus Fabia, Walker, Mon. Chal. ii. 33.

a. King Greorge's Sound, Australia. Presented by C. Darwin, Esq.

Pteromalus Gorgias, Walker, Mon. Chal. ii. 34.

a. Sydney, New South Wales. Presented by C. Darwin, Esı.

Pteromalus Cosis, Walker, Mon. Chal. ii. 68.

a. Bahia, Brazil. Presented by C. Darwin, Esq.

Pteromalus Driopides, Walker, Mon. Chal. ii. 68.

a. Bahia, Brazil. Presented by C. Darwin, Esq.

Pteromalus Prothous, Walker, Mon. Chal. ii. 87.

a. Island of Chiloe, Chili. Presented by C. Darwin, Esq.

Pteromalus Mydon, Walker, Mon. Chal. ii. 87.

a. Island of Chiloe, Chili. Presented by C. Darwin, Esq.

Pteromalus Traulus, Walker, Mon. Chal. ii. 88.

a. Island of Chiloe, Chili. Presented by C. Darwin, Esq.

Pteromalus Rhæo, Walker, Mon. Chal.ii. 88.

a. Island of Chiloe, Chili. Presented by C. Darwin, Esq.

Pteromalus Vulso, Walker, Mon. Chal. ii. 89.

a. Island of Chiloe, Chili. Presented by C. Darwin, Esq.

Pteromalus Lelex, Walker, Mon. Chal. ii. 95.

a. New Zealand. Presented by C. Darwin, Esq.

Pteromalus Calenus, Walker, Ann. Nat. Hist. xi. 31.

a. Concepcion, Chili. Presented by C. Darwin, Esq.

Preromalus Toxeus, Walker, Ann. Nat. Hist. xi. 186.

a. Coquimbo, Chili. Presented by C. Darwin, Esq.

Pteromalus Sestius, Walker, Ann. Nat. Hist. xi. 186.

a. Coquimbo, Chili. Presented by C. Darwin, Esq. 
Preromalus Rhoebus, Walker, Ann. Nat. Hist. xi. 187.

a. Coquimbo, Chili. Presented by C. Darwin, Esq.

Pteromalus, Iambe, Walker, Mon. Chal. ii. 95.

a. New Zealand. Presented by C. Darwin, Esq.

Pteromalus Ipsea, Walker, Mon. Chal. ii. 97.

a. St. Helena. Presented by C. Darwin, Esq.

Pteromalus Helice, Walker, Ann. Nat. Hist. xii. 46.

a. St. Vincent's Island, West Indies. Presented by F. Walker,

Esq.

Pteromalus? Oxynthes, Walker, Ann. Nat. Hist. xi. 184.

a. Island of Chonos, Chili. Presented by C. Darwin, Esq.

\section{Hetroxys, Westwood.}

Hetroxys pulcherrimus, Westwood, Mag. Nat. Hist. v. 121.

a. England. Presented by F. Walker, Esq.

Hetroxys scenicus. Pteromalus scenicus, Walker, Ent. Mag. iv.

10. Entomologist, pl. G. f. 1. Diplolepis bicolorata, Spinola.

Ins. Lig. Fasc. iv. 221? Cleonymus bicoloratus, Spin. Ann.

Mus. vii. 149.

a. England. Presented by F. Walker, Esq.

Hetroxys stenogaster. Pteromalus stenogaster, Walker, Ent.

Mag. iv. 11.

a. England. Presented by F. Walker, Esq.

Hetroxys fuscescens. Pteromalus fuscescens, Walker, Ent. Mag. iv. 12.

a. England. Presented by F. Walker, Esq.

32. Cheiropachus, Westwood.

Cheiropachus quadrum, Westwood, Zool. Journ. iv. pl. 2. f. 2. Ichneumon quadrum, Fab. Ent. Syst. ii. 186, 219. Diplolepis quadrum, Fab. Syst. Piez. 152, 16. Spinola, Ins. Lig. Fasc. iii. 161, 14. Cynips quadrum, Boyer de Fonscol. Ann. Sci. Nat. 1832, 298, 1. Pteromalus bimaculatus, Nees. Hym. Ieh. aff. Mon. ii. 96? Cleonymus maculipennis, Curtis, Brit. Ent. iv. 194.

a. England. Presented by F. Walker, Esq.

b. France. Presented by M. Brebisson.

Cheiropachus tutela, Walker, Ent. Mag. iv. 14.

a. England. Presenterl by F. Walker, Esq. 
33. Macroneura, Walker.

Macroneura maculipes, Walker, Ent. Mag. iv. 354. Entomologist, pl. O. f. 3.

a. England. Presented by F. Walker, Esq.

34. Prosopon, Walker.

Prosopon montanum, Walker, Ent. Mag. iv. 356. Entomologist, $p l$. D. $f .4$.

a. England. Presented by F. Walker, Esq.

35. Trigonoderus, Westwood.

Trigonoderus pulcher, Westwood, Lond. and Edin. Phil. May.

Third Series, i. 127. Walker, Ent. Mag. iv. 16.

a. England. Presented by F. Walker, Esq.

Trigonoderus filatus, Walker, Ent. Mag.iv. 17.

a. England. Presented by F. Walker, Esq.

Trigonoderus ductilis, Walker, Ent. Mag. iv. 17.

a. England. Presented by F. Walker, Esq.

Trigonoderus affinis, Walker, Ent. Mag. iv. 19.

a. England. Presented by F. Walker, Esq.

Trigonoderus linearis, Walker, Ent. Mag. iv. 19.

a. England. Presented by F. Walker, Esq.

Trigonoderus figuratus, Walker, Ent. Mag. iv. 20.

a. England. Presented by F. Walker, Esq.

Trigonoderus elegans, Walker, Ent. Mag. iv. 21.

a. England. Presented by F. Walker, Esq.

Trigonoderus obscurus, Walker, Ent. Mag.iv. 21. Entomologist, pl. E. $f .1$.

a. England. Presented by F. Walker, Esq.

Trigonoderus deductor, Walker, Ent. Mag.iv. 20.

a. England. Presented by F. Walker, Esq.

Trigonoderus dolosus, Walker, Ent. Mag. iv. 23,

a. England. Presented by F. Walker, Esq.

Trigonoderus hirticornis, Walker, Ent. Mag. iv. 23.

a. England. Presented by F. Walker, Esq.

36. Merostenus, Walker.

Merostenus Phedyma, Walker, Ent. Mag. iv. 355. Entomologist, pl. C. $f .4$.

a. England. Presented by F. Walker, Esq. 


\section{Lelaps, Haliday.}

Le Laps Sadales, Haliday, Trans. Ent. Soc. iii. 292. Merostenus Sadales, Walker, Mon. Chal. ii. 93.

a. Charles' Island, Galapagos. Presented by C. Darwin, Esq.

Le La PS pulchricornis, Haliday, Trans. Ent. Soc. iii. 300.

a. St. Vincent's Isle, West Indies. Presented by the Entomological Club.

\section{Epistenia, Westwood.}

Epistenia cæruleata, Westwood. Griffith's Anim. Kingdom, pl. 77. f. 3.

a. Georgia, North America. From Mr. Abbot's collection.

Epistenia Feretrius. See Appendix.

a. Philippine Islands. From Mr. Wood's collection.

Fam. 9. Eupelmide.

\section{Cleonymus, Latreille.}

Cleonymus depressus, Latreille, Gen. Crust. et Ins. iv. 29. Ichneumon depressus, Fab. Ent. Syst. Suppl. 231, 220, 221. Coqueb. Illus. Icon. i. 21. Tab. 5. f. 5. Diplolepis depressa, Fab. Syst. Piez. 151. 13. Spin. Ins. Lig. Fasc. iv. 220. Сynips depressus, Lamarck, Hist. Nat. Anim. sans Vertebr. iv. $156,7$.

a. England. Presented by F. Walker, Esq.

2. Calosoter, Walker.

CALosoter vernalis, Walker, Ent. Mag. iv, 359. Entomologist, pl. O.f. 2.

a. England. Presented by F. Walker, Esq.

Calosoter æstivalis, Walker, Ent. Mag. iv. 35.9

a. England. Presented by F. Walker, Esq.

Calosoter Eneubulus, Walker, Ent. Mag. v. 475.

a. Charles' Island, Galapagos. Presented by C. Darwin, Esq.

Calosoter Anemetus. See Appendix.

a. Philippine Islands. From Mr. Wond's collection. 


\section{Eupelmus, Dalman.}

Eupelmus urozonus, Dalman, Kongl. Vet. Acad. Handl. 1820. ii. 378, 2. Tab. 8.f. 34, 37 .

a. England. Presented by F. Walker, Esq.

b. Sweden. Presented by F. Walker, Esq.

c. South of France. Presented by the Entomological Club.

d. Sydney, New South Wales. Presented by C. Darwin, Esq.

Eupelmus Degeeri, Dalman, Konyl. Vet. Acad. Handl. 1820, ii. 379, 3. Entomologist, pl. F.f. 2. Ichneumon vesicularis, De Geer, ii. 909. Tab. 31.f. 22.- Diplolepis vesicularis, Spinolit, Ins.Lig. Fasc. iii. 161, 13.

a. England. Presented by F. Walker, Esq.

b. Sweden. Presented by F. Walker, Esq.

c. Fontainbleau, France. Presented by the Entomological Club. Eupelmus atropurpureus, Dalman, Kongl. Vet. Acad. Handl. 1820 , ii. 381,4 .

a. Sweden. Presented by F. Walker, Esq.

Eupelmus Amillarus, Walker, Ent. Mag. v. 475.

a. Bahia, Brazil. Presented by C. Darwin, Esq.

Eupelmus Dodone. Calosoter Dodone, Walker, Mon. Chal. ii. 35 .

a. King George's Sound, Australia. Presented by C. Darwin, Esq.

4. Metapelma, Westwood.

Metapelma spectabilis, Westwood, Zool. Soc. Proc. Pt. 3, 1835 69.

a. Georgia, North America. From Mr. Abbot's collection.

Fam. 10. Encyrtide.

1. Ericydnus, Haliday.

Ericydnus strigosus. Eucyrtus strigosus, Nees. Hym. Ich aff. Mon. ii. 227. Walker, Ent. Mag. iv. 364. Entomologist, pl. H. f. 1.

a. England Presented by F. Walker, Esq.

Ericydnus chryscus. Pteromalus? chryscus, Walker, Mon. C'hal. ii. 34.

b. King George's Sound, Australia. Presented by C. Darwir, Esq. 
Ericydnus Megalarus. Eulophus Megalarus, Walker, Ent. Mag. v. 477.

a. King George's Sound, Australia. Presented by C. Darwin, Esq.

\section{Cheiloneurus, Westwood.}

Cheiloneurus elegans, Westwood, Lond. and Edin. Phil. Mag. iii. 343. Encyrtus Paralia, Walker, Ent. Mag. iv. 446. Var. E. argentifer, Haliday, Ent. Mag. iv.444. E. elegans, Dalm. Kongl. Vet. Acad. Handl. 1820, 151. Eupelmus elegans, Dalm. Kongl., \&c. 1820, 384. Cleonymus elegans, Nees. Hym. 1ch. aff. Mon. ii. 88.

a. Fontainbleau, France. Presented by the Entomological Club. b. Sweden. Presented by F. Walker, Esq.

\section{Cerchysius, Westwood.}

Cerchysius urocerus, Westwood, Lond. and Edin. Phil. Mag. Third Series, i. 127. Entomologist, pl. H. f. 3. C. stigmaticalis Var. Westwood, Lond. and Edin. Phil. Mag. i. 127. Encyrtus urocerus, Dalm. Kongl. Vet. Acad. Handl. 1823,368.

E. caudatus, Foerster, Mon. Pterom. i. 45.

a. England Presented by F. Walker, Esq.

b. Sweden. Presented by F. Walker, Esq.

\section{Cercobelus, Haliday.}

Cercobelus Jugœus. Encyrtus Jugœus, Walker, Ent. May. v. 48. Entomologist, pl. N.f. 1.

a. England. Presented by F. Walker, Esq.

\section{Encyrtus, Dalman.}

Encyrtus cyaneus, Dalm. Kongl. Vet. Acad. Handl. 1820, 160.

a. England. Presented by F. Walker, Esq.

b. Sweden. Presented by F. Walker, Esq.

Ency rtus Batillus, Walker, Ent. Mag, iv. 442.

a. England. Presented by F. Walker, Esq.

b. Fontainbleau, France. Presented by the Entomological Club.

$c$. Clermont, France. Presented by the Entomological Club.

Encyrtus Celadus, Walker, Ent. Mag. v. 427.

a. Fontainbleau, France. Presented by F. Walker, Esq.

Encyrtus Fadus, Walker, Ent. Mag.v. 429.

a. South of France. Presented by the Entomological Club.

b. Fontainbleau, France. Presented by the Entomological Club. 
Encyrtus Gabinius, Walker, Ent. Mag.iv. 443.

a. England. Presented by F. Walker, Esq.

Encyrtus Imandes, Walker, Ent. Mag. iv. 449.

a. England. Presented by F. Walker, Esq.

Encyrtus fuscipennis, Dalm. Kong?. Vet. Acad. Handl. 1820, 354.

a. Sweden. Presented by F. Walker, Esq.

Encyrtus hemipterus, Dalm. Kongl. Vet. Acad. Handl, 1820. 160.

a. England. Presented by F. Walker, Esq.

b. Sweden. Presented by F. Walker, Esq.

c. Auvergne, France. Presented by the Entomological Club.

Encyrtus Lindus, Walker, Ent. Mag. iv. $45 i$.

a. England. Presented by F. Walker, Esq.

Encyrtus Anceus, Walker, Ent. Mag. iv. 452.

a. England. Presented by F. Walker, Esq.

Encyrtus melanopus, Haliday, MSS. Walker, Ent. Mag. iv. 453.

a. England. Presented by F. Walker, Esq.

Encyrtus Glaphyra, Walker, Ent. Mag. iv. 454.

a. England. Presented by F. Walker, Esq.

Ency rtus serricornis, Dalm. Kongl. Vet. Acad. Handl. 1820.

a. England. Presented by F. Walker, Esq.

Encyrtus Aralius, Walker, Ent. Mag. iv. 457.

a. England. Presented by F. Walker, Esq.

Encrrtus Teuteus, Walker, Ent. Mag. iv. 458.

a. England. Presented by F. Walker Esq.

b. Fontainbleau, France. Presented by the Entomological Clab.

Encyrtus Spherus, Walker, Ent. Mag. iv. 459.

a. England. Presented by F. Walker, Esq.

Encyrtus subcupratus, Dalm. Kongl. Vet. Acad. Handl. 1820.

a. England. Presented by F. Walker, Esq.

b. Clermont, France. Presented by the Entomological Club.

Encyrtus Tiliaris, Dalm. Kongl. Vet. Acad. Handl. 1820, 171.

E. coniferæ, Haliday, MSS. Walker, Ent. Mag. iv. 461.

a. England. Presented by F. Walker, Esq.

b. Sweden. Presented by F. Walker, Esq.

Encrrtus barbarus, Dalm. Kongl. Vet. Acad. Handl. 1820, 158.

a. Sweden. Presented by F. Walker, Esq.

Encrrtus Anebus, Walker, Ent. Mag. iv. 457.

a. Fontainbleau, France. Presented by the Entomological Club. 
Encyrtus subplanus, Dalm. Kongl. Vet. Acad. Handl. 1820, 362. a. Sweden. Presented by F. Walker, Esq.

Encyrtus Aithyia, Walker, Ent. Mag. iv. 458.

a. Fontainbleau, France. Presented by the Entomological Club.

Encyrtus chalconotus, Dalm. Kongl. Vet. Acad. Handl. 1820, 169. E. Mitreus, Walker, Ent. Mag. v. 35.

a. England. Presented by F. Walker, Esq.

b. Sweden. Presented by F. Walker, Esq.

Encyrtus Phithra, Walker, Ent. Mag. v. 36.

a. England. Presented by F. Walker, Esq.

Encyrtus geniculatus, Dalm. Kongl. Vet. Acad. Handl. 1820, 361.

b. Sweden. Presented by F. Walker, Esq.

Encyrtus truncatellus, Dalm. Kongl. Vet. Acad. Handl. 1820, 168.

a. England, Presented by F. Walker, Esq.

b. Fontainbleau, France. Presented by the Entomological Club.

c. Sweden. Presented by F. Walker, Esq.

Encyrtus Liriope, Walker, Ent. Mag. v. 40.

a. England. Presented by F. Walker, Esq.

b. Auvergne, France. Presented by the Entomological Cluh.

Encyrtus chalcostomus, Dalm. Kongl. Vet. Acad. Handl. 1820, 341.

a. Sweden. Presented by F. Walker, Esq.

Encyrtus interpunctus, Dalm. Kongl. Vet. Acad. Handl. 1820, 157.

a. Sweden. Presented by F. Walker, Esq.

Encyrtus cyanifrons, Dalm. Kongl. Vet. Acad. Handl. 1820, 159.

a. Sweden. Presented by F. Walker, Esq.

Encyrtus sylvius, Dalm. Kongl. Vet. Acad. Handl. 1820. 154.

a. Sweden. Presented by F. Walker, Esq.

Encyrtus paradoxus, Dalm. Kongl. Vet. Acad. Handl. 1820, 163.

a. Sweden. Presented by F. Walker, Esq.

Encyrtus filicornis, Dalm. Kongl. Vet. Acad. Handl. 1820, 351

E. Didius, Walker, Ent. Mag. iv. 452.

a. England. 'Presented by F. Walker, Esq.

b. Sweden. Presented by F. Walker, Esq.

c. Fontainbleau, France. Presented by the Entomological Club.

Encyrtus Swederi, Dalm. Kongl. Vet. Acad. Handl. 1820, 152.

E. lunatus, Dalm. Kongl. Vet. Acad. Handl. 1820, 164. E.

hirticornis, Dalm. Kongl. Vet. Acad. Handl. 1820. E. Vitis,

Curtis. Brit. Ent. 395. 
a. England. Presented by F. Walker, Esq.

b. Sweden. Presented by F. Walker, Esq.

c. France. Presented by the Entomological Club.

Encyrtus tessellatus, Dalm. Kongl. Vet. Acad. Handl. 1820, 342.

a. England. Presented by F. Walker, Esq.

Encyrtus æruginosus, Dalm. Kongl. Vet. Acad. Handl. 1820, 170.

a. Sweden. Presented by F. Walker, Esq.

Encyrtus flaminius, Dalm. Kongl. Vet. Acad. Handl. 1820, 340.

a. Sweden. Presented by F. Walker, Esq.

b. France. Presented by the Entomological Club.

Ency Rtus Erginus, Walker, Ent. Mag. v. 53.'

a. England. Presented by F. Walker, Esq.

Encyrtus scutellaris, Dalm. Kongl. Vet. Acad. Handl. 1820, 150.

Pteromalus scutellatus, Suederus, Kongl. Vet., \&c. 1795.

a. England. Presented by F. Walker, Esq.

b. Sweden. Presented by F. Walker, Esq.

c. France. Presented by the Entomological Club,

Ency rtus obscurus, Dalm. Kongl. Vet. Acad. Handl. 1820, 164.

a. Sweden. Presented by F. Walker, Esq.

Encyrtus Morio, Dalm. Kongl. Vet. Acad. Handl. 1820, 164.

a. Sweden. Presented by F. Walker, Esq.

Ency rtus apicalis, Dalm. Kongl. Vet. Acad. Handl. 1820, 153.

a. England. Presented by F. Walker, Esq.

b. Sweden. Presented by F. Walker, Esq.

Encyrtus sericeus, Dalm. Kongl. Vet. Acad. Handl. 1820, 357.

a. England. Presented by F. Walker, Esq.

b. Sweden. Presented by F. Walker, Esq.

c. France. Presented by M. Brebisson.

Encyrtus Pappus, Walker, Ent. Mag. v. 107.

a. England. Presented by F. Walker, Esq.

Ency rtus fulvifrons, Haliday, Ent. Mag. v. 109.

a. England. Presented by F. Walker, Esq.

b. Sweden. Presented by F. Walker, Esq.

Encyrtus punctipes, Dalm. Kongl. Vet. Acad. Handl. 1820, 154.

a. England. Presented by F. Walker, Esq.

b. Sweden. Presented by F. Walker, Esq.

Encyrtus Bohemanni, Westwood, Lond. and Edin. Phil. Mag.

Third Series, x. 63, 441.

a. England. Presented by F. Walker, Esq. 
Encyrtus Zetterstedii, Westwood, Lond. and Edin. Phil. Mag. Third Series, x. 63.440.

a. England. Presented by F. Walker, Esq.

Ency rtus Scythis, Walker, Ent. Mag. v. 118.

a. England. Presented by F. Walker, Esq.

$b$. Fontainbleau, France. Presented by the Entomological Club.

Encyrtus fusciollis, Dalm. Kongl. Vet. Acad. Handl. 1820.

a. England. Presented by F. Walker, Esq.

Encyrtus atricollis, Dalm. Kongl. Vet. Acad. Handl: 1820, 358.

a. Sweden. Presented by F. Walker, Esq.

Encyrtus nubilipennis, Curtis' Guide. Walker, Ent. Mag. v. 113.

E. Dalmanni? Westwood, Lond. and Edin. Phil. Mag, Third

Series, x. 440.

a. England. Presented by F. Walker, Esq.

Encyrtus Lambinus, Walker, Ent. Mag. v. 422.

a. England. Presented by F. Walker, Esq.

Encyrtus Cypris, Walker, Ent. Mag. v. 419.

a. England. Presented by F. Walker, Esq.

Encyrtus Thebe, Walker, Ent. Mag. v. 418.

a. England. Presented by F. Walker, Esq.

Ency Rtus Ilithyia, Walker, Ent. Mag. v, 418.

a. England. Presented by F. Walker, Esq.

Encyrtus Elpis, Walker, Ent. Mag. v. 117.

a. England. Presented by F. Walker, Esq.

Encyrtus Piso, Walker, Ent: Mag. v. 423.

a. England. Presented by F. Walker, Esq.

Encyrtus Mysus, Walker, Ent. Mag. v. 424.

a. England. Presented by F. Walker, Esq.

Ency rtus Aretas, Walker, Ent. Mag. v. 425.

a. England. Presented by F. Walker, Esq.

Ency rtus Syliæus, Walker, Ent. Mag. v. 426.

a. England. Presented by F. Walker, Esq.

Encyrtus Euryclea, Walker, Ann. Nat. Hist, xiv. 184.

a. England. Presented by F. Walker, Esq.

Encyrtus æstivus, Dalm. Kongl. Vet. Acad. Handl. 1820, 368.

E. Cleone, Walker, Ann. Nat. Hist. xiv. 407.

a. Sweden. Presented by F. Walker, Esq.

Encyrtus Zephyrinus, Dalm. Kongl. Vet. Acad. Handl. 1820, 167.

a. Sweden. Presented by F. Walker, Esq. 
Encyrtus Odacon, Walker, Ent. Mag.v. 476.

a. Hobart Town, Van Diemen's Land. Presented by C. Darwin, Esq.

Ency Rtus Gargaris, Walker, Ann. Nat. Hist. xii. 47.

a. St. Vincent's Island, West Indies. Presented by F. Walker, Esq.

Encyrtus Bolus, Walker, Ann. Nat. Hist. xiv. 17.

Martin's Falls, Albany River, Hudson's Bay. Presented by G, Barnston, Esq.

Encyrtus Zebina, Walker, Mon, Chal. ii. 36.

a. Hobart Town, Van Diemen's Land. Presented by C. Darwin, Esq.

Excyrtus Lucetius, Walker, Mon. Chal. ii. 36.

a. Hobart Town, Van Diemen's Land. Presented by C. Darwin, Esq.

b. King George's Sound, Australia. Presented by C. Darwin, Esq.

Encrrtus Salacon, Walker, Mon. Chal. ii. 37.

a. Hobart Town, Van Diemen's Land. Presented by C. Darwin, Esq.

Encyrtus Cheles, Walker. Mon. Chal, ii. 37.

a. Hobart Town, Van Diemen's Land. Presented by C. Darwin, Esq.

Encyrtus Arsanes, Walker, Mon. Chal. ii. 38.

a. Hobart Town, Van Diemen's Land. Presented by C. Darwin, Esq.

Encyrtus Xuthus, Walker, Mon. Chal. ii. 38.

a. Hobart Town, Van Diemen's Land. Presented by C. Darwin, Esq.

b. King George's Sound, Australia. Presented by C. Darwin, Esq.

Encretus Pacorus, Walker, Mon. Chal. ii. 39.

a. Sydney, New South Wales. Presented by C. Darwin, Esq.

Encyrtus Zameis, Walker, Mon. Chal. ii. 39.

a. King George's Sound, Australia. Presented by C. Darwin, Esq.

Encyrtus Epytus, Walker, Mon. Chal. ii. 69.

a. Bahia, Brazil. Presented by C. Darwin, Esq.

Encrrtus ineptus, Dalm. Kongl. Vet. Acad. Handl. 1820, 367.

Sphenolepis inepta, Nees. d'Ess. Hym. Ich. aff. Mon. ii. 258.

Choreius ineptus, Westwood, Lond. and Edin. Phil. Mag.

Third Series. x. 63, 442. Choreia nigro-æne:, Westwood, 
Mag. Nat. Hist. vi. 122. Crantor, Haliday, Ent. Mag. i. 268.

a. England. Presented by F. Walker, Esq.

b. Sweden. Presented by F. Walker, Esq.

\section{Aphelinus, Dalman.}

Aphelinus basalis, Walker, Mon. Chal. i. 2. Entomologist, pl. K. f. 2. Agonioneurus basalis, Westwood, Loudon's Mag. Nat. Hist. vi. 122. Myina facialis, Foerster, Pter $\Theta m$. i. 44, 7. a. England. Presented by F. Walker, Esq.

Aphelinus Asychis, Walker, Mon. Chal. i. 2. a. England. Presented by F. Walker, Esq.

Aphelinus Euthria, Walker, Mon. Chal.i. 3. Myina abdominalis? Nees. ab Ess. Hym. Ich. aff. Mon. ii. 190. Myina fusciscapus, Foerster, Mon. Pterom. i. 45, 8.

a. England. Presented by F. Walker, Esq.

Aphelinus, Chaonia, Walker, Mon. Chal. i. 4. Myina varipes, Foerster, Mon. Pterom. i. 45, 9. Myina tibialis? Nees. ab Ess. Hym. Ich. aff. Mon. ii. 191.

a. England. Presented by F. Walker, Esq.

b. Fontainbleau, France. Presented by the Entomological Club.

Aphelinus Mæris, Walker, Mon. Chal. i. 5.

a. England. Presented by F. Walker, Esq.

b. France. Presented by the Entomological Club.

Aphelinus Proclia, Walker, Mon. Chal. i. 9.

a. England. Presented by F. Walker, Esq.

Aphelinus Inaron, Walker, Mon. Chal. i. 10.

a. Fontainbleau, France. Presented by the Entomological Club.

Aphelinus Lycimnia, Walker. Mon. Chal. i. 11. Myina semicircularis, Foerster, Monogr. Pterom. i. 44, 2.

a. France. Presented by F. Walker, Esq.

\section{Coccophagus, Westwood.}

Coccophagus scutellaris, Walker, Mon. Chal. i. 6. Entomologist, pl. K. f. 1. Entedon scutellaris, Dalm. Kongl. Vet. Acad. Handl. 1825, 365. Coccophagus pulchellus, Westwood, Lond. and Edin. Phil. Mag. iii. 1833. Eulophus flavovarius, Nees. ab. Ess. Hym. Ich. aff. Mon. ii. 164, 428.

a. England. Presented by F. Walker, Esq.

b. Sweden. Presented by F. Walker, Esq.

Coccophagus insidiator. Eutedon insidiator, Dalm. Kongl. Vet. Acad. Handl. 1825, 365. Cocophagus obscurus, Westwood, 
Lond. and Edin. Phil. Mag. iii. 1833. Eulophus impeditus,

Nees. ab. Ess. Hym. Ich. aff. Mon. ii. 178, 438.

a. England. Presented by F. Walker, Esq.

b. Sweden. Presented by F. Walker, Esq.

c. France. Presented by the Entomological Club.

Fam. 11. Eulophid

1. Trichogramma, Westwood.

Trichogramma evanescens, Westwood, Lond. and Edin. Phil. Mag. ii. 12, 144. Entomologist, pl. K. f. 4. Calleptiles latipennis, Haliday, Ent. Mag. i. 339. Pteroptrix evanescens, Walker, Mon. Chal. i. 13.

a. England. Presented by F. Walker, Esq.

\section{Pteroptrix, Westwood.}

Pteroptrix dimidiatus, Westwood, Lond. and Edin. Phil. Mag. iii. 344 .

a. England. Presented by F. Walker, Esq.

Pteroptrix Doricha, Walker, Mon. Chal. i. 15.

a. England. Presented by F. Walker, Esq.

Pteroptrix Menes, Walker, Mon. Chal.i. 18. Entomologist, pl.

M. $f .3$.

a. England. Presented by F. Walker, Esq.

Pteroptrix Celsus, Walker, Mon. Chal. i. 16.

a. Paris. Presented by F. Walker, Esq.

\section{Elasmus, Westwood.}

Elasmus flabellatus, Westwood, Lond. and Edin. Phil. Mag. Third Series, iii. 17, 343, 1833 . Eulophus flabellatus, Boyer de Fonscol. Ann. Sci. Nat. 1832, 298. Aneure rhipicerus, Foerster, Mon. Pteromal. i. 45. f. 16.

a. England. Presented by J. C. Dale, Esq.

Elasmus Anius. See Appendix.

a. England. Presented by F. Walker, Esq.

4. Entedon, Dalman.

Entedon Ochus, Walker, Mon. Chal. i. 21. a. England. Presented by F. Walker, Esq. Entedon Acestor, Walker, Mon. Chal. i. 21. a. England. Presented by F. Walker, Esq. 
Entedon Lanassa, Walker, Mon. Chal. i. 22. a. England. Presented by F. Walker, Esq.

Ente don formosus, Walker, Mon. Chal. i. 26. Closterocerus formosus, Westwood, Mag. Nat. Hist. vi. 420.

a. England. Presented by F. Walker, Esq.

Entedon trifasciatus, Walker, Mon. Chal.i. 26. Closterocerus trifasciatus, Westwood, Loudon's Mag. Nat. Hist. vi. 420. Eulophus bifasciatus, Nees. ab Ess. Hym. Ich. aff. Mon. ii. $156,6$.

a. England. Presented by F. Walker, Esq.

b. Sweden. Presented by F. Walker, Esq.

Entedon Cercius. Closterocerus Cercius, Walker, Ann. Nat. Hist. xi. 31.

a. Concepcion, Chili. Presented by C. Darwin, Esq.

Entedon Xenodice. Closterocerus Xenodice, Walker, Ann. Nat. Hist. x. 273.

a. Valdivia, Chili. Presented by C. Darwin, Esq.

Entedon Pelor. Closterocerus Pelor, Walker, Ann. Nat. Hist. xi. 185.

a. Island of Chonos, Chili. Presented by C. Darwin, Esq.

Entenon Ufens, Walker, Ann. Nat. Hist. xi. 184.

a. Island of Chonos, Chili. Presented by C. Darwin, Esq.

Entedon Agamedes, Walker, Mon. Chal. i. 29.

a. England. Presented by F. Walker, Esq.

Entedon gemmeus, Walker, Mon. Chal. i. 30. Derostenus gemmeus, Westwood, Loudon's Mag. Nat. Hist. vi. 495.

a. England. Presented by F. Walker, Esq.

b. Sweden. Presented by F. Walker, Esq.

Entedon Latreillii, Walker, Mon. Chal. i. 31. Eulophus Latreillii, Curtis, Brit. Ent. iii. 133.

a. England. Presented by F. Walker, Esq.

Entedon Atys, Walker, Mon. Chal. i. 34.

a. Englaud. Presented by F. Walker, Esq.

Entedon Metella, Walker, Mon. Chal.i. 35.

a. England. Presented by F. Walker, Esq.

Entedon Varus, Walker, Mon. Chal. i. 35.

a. England. Presented by F. Walker, Esq.

Entedon Pentheus, Walker, Mon. Chal. i. 38.

a. England. Presented by F. Walker, Esq.

Entedon Amanus, Walker, Mon. Chal.i. 39.

a. England. Presented by F. Walker, Esq.

b. South of France. Presented by the Entomological Club. 
Entedon Polyzo, Walker, Mon. Chal i. 40. a. England. Presented by F. Walker, Esq. Entedon Acerbas, Walker, Mon. Chal. i. 40. a. England. Presented by F. Walker, Esq. Entedon Prodice, Walker, Mon. Chal. i. 41. a. England. Presented by F. Walker, Esq. Entedon Amyclas, Walker, Mon. Chal. i. 42. Entomologist, pl. L. $f .4$.

a. England. Presented by F. Walker, Esq. Entedon Daunus, Walker, Mon. Chal.i. 43. a. England. Presented by F. Walker, Esq. Entedon Assis, Walker, Mon. Chal. i. 43. a. England. Presented by F. Walker, Esq. Entedon Erigone, Walker, Mon. Chal. i. 44. a. England. Presented by F. Walker, Esq. Entedon Larina, Walker, Mon. Chal. i. 46. a. England. Presented by F. Walker, Esq. Entedon Nœnia, Walker, Mon. Chal. i. 48. a. England. Presented by F. Walker, Esq. Entedon Tamus, Walker, Mon. Chal. i. 49. a. England. Presented by F. Walker, Esq. Entedon Laomedon, Walker, Mon. Chal. i. 50. a. England. Presented by F. Walker, Esq. Entedon Matho, Walker, Mon. Chal.i. 51. a. England. Presented by F. Walker, Esq. Entedon Inarus, Walker, Mon. Chal. i. 52. a. England. Presented by F. Walker, Esq. Entedon Nitetis, Walker, Mon. Chal. i. 52. a. England. Presented by F. Walker, Esq. Entedon Sartamus, Walker, Mon. Chal.i. 54. a. England. Presented by F. Walker, Esq.

Entedon gemma, Curtis, Walker, Mon. Chal. i. 53.

a. England. Presented by F. Walker, Esq.

Entedon Novellus, Walker, Mon. Chal. i. 55. a. England. Presented by C. Walker, Esq. Entedon Amyrtœus, Walker, Mon. Chal. i. 57. a. England. Presented by F. Walker, Esq. Entedon Cydon, Walker, Mon. Chal. i. 58. a. England. Presented by F. Walker, Esq. b. Fontainbleau, France. Presented by the Entomological Club. 
Entedon Acoris, Walker, Mon. Chal. i. 59.

b. Clermont, France. Presented by the Entomological Club.

Entedon Calitor, Walker, Mon. Chal. i. 60.

a. England. Presented by F. Walker, Esq.

Entedon Bibulus, Walker. Mon. Chal. i. 62. a. England. Presented by F. Walker, Esq.

Entedon Amyite, Walker, Mon. Chal.i. 65.

a. England. Presented by F. Walker, Esq.

b. Alten, Finmark. Presented by F. Walker, Esq.

Entedon Enephes, Walker, Mon. Chal. i. 67.

a. England. Presented by F. Walker, Esq.

Entedon Eutropius Walker, Mon. Chal.i. 69.

a. England. Presented by F. Walker, Esq.

Entedon Altadas, Walker, Mon. Chal. i. 70.

a. England. Presented by F. Walker, Esq.

b. Alten, Finmark. Presented by F. Walker, Esq.

Entedon Parmys, Walker, Mon. Chal. i. 71. a. England. Presented by F. Walker, Esq.

Entedon Lycoris, Walker, Mon. Chal. i. 72.

a. England. Presented by F. Walker, Esq.

Entedon Lycambes, Walker, Mon. Chat. i. 73.

a. England. Presented by F. Walker, Esq.

Entedon Zanara, Walker, Mon. Chal. i. 95.

a. England. Presented by F. Walker, Esq.

Entedon Mera, Walker, Mon. Chal. i. 97.

a. England. Presented by F. Walker, Esq.

Entedon Busiris, Walker, Mon. Chal. i. 99.

a. England. Presented by F. Walker, Esq.

b. Sweden. Presented by F. Walker, Esq.

Entedon Merion, Walker, Mon. Chal.i. 100.

a. England. Presented by F. Walker, Esq.

Entedon Diotimus, Walker, Mon. Chal. i. 101.

a. England. Presented by F. Walker, Esq.

Entedon Pharnus, Walker, Mon. Chal. i. 102.

a. England. Presented by F. Walker, Esq.

Entedon Argon, Walker, Mon. Chal. i. 102.

a. England. Presented by F. Walker, Esq.

Entedon Sparetus, Walker, Mon. Chal.i. 103.

a. England. Presented by F. Walker, Esq.

Entedon Hercyna, Walker, Mon. Chal. i. 104.

a. England. Presented by F. Walker, Esq. 
Entedon Euphorion, Walker, Mon. Chal.i. 105.

a. England. Presented by F. Walker, Esq.

Entedon Nephthe, Walker, Mon. Chal. i. 108.

a. England. Presented by F. Walker, Esq.

Entedon Arisba, Walker, Mon. Chal. i. 121.

a. England. Presented by F. Walker, Esq.

b. Sweden. Presented by F. Walker, Esq.

Entedon Chthonia, Walker, Mon. Chal. i. 122.

a. England. Presented by F. Walker, Esq.

Entedon Bedius, Walker, Ann, Nat. Hist. x. 115.

a. Valparaiso, Chili. Presented by C. Darwin, Esq.

Entedon Flacilla, Walker, Ann. Nat. Hist. x. 115.

a. Valparaiso, Chili. Presented by C. Darwin, Esq.

Entedon Eubius, Walker, Mon. Chal.i. 109.

a. England. Presented by F. Walker, Esq.

Entedon Amyntas, Walker, Mon. Chal. i. 111.

a. England. Presented by F. Walker, Esq.

Entedon Epigonus, Walker, Mon. Chal. i. 112.

a. England. Presented by F. Walker, Esq.

b. Alten, Finmark. Presented by F. Walker, Esq.

Entedon Coenus, Walker, Mon. Chal. i. 113.

a. England. Presented by F. Walker, Esq.

Entedon Saulius, Walker, Mon. Chal. i. 115.

a. England. Presented by F. Walker, Esq.

Entedon Corytus, Walker, Mon. Chal. i. 116.

a. England. Presented by F. Walker, Esq.

Entedon Sosarmus, Walker, Mon. Chal. i. 116.

a. England. Presented by F. Walker, Esq.

b. Fontainbleau, France. Presented by the Entomological Club.

Entedon Clita, Walker, Mon. Chal. i, 117.

a. England. Presented by F. Walker, Esq.

Entedon Pyrgo, Walker, Mon. Chal. i. 119.

a. England. Presented by F. Walker, Esq.

Entedon Lysis, Walker, Mon. Chal. i. 114.

a. England. Presented by F. Walker, Esq.

Entedon Telephe, Walker, Mon. Chal.i. 75.

a. England. Presented by F. Walker, Esq.

Entedon admirabilis. Smaragdites admirabilis, Westwood, Mag.

Nat. Hist. vi. 35, 418. Omphale Salicis, Haliday, Ent. Maig.

i. 339. Entedon Salicis, Walker, Mon. Chal. i. 76.

a. England. Presented by F. Walker, Esq. 
Entedon Etius, Walker, Mon. Chal.i. 78.

a. England. Presented by F. Walker, Esq.

b. Alten, Finmark. Presented by F. Walker, Esq.

Entedon Theana, Walker, Mon. Chal. i. 81.

a. England. Presented by F. Walker, Esq.

Entedon Ithonus, Walker, Mon. Chal.i. 82.

a. England. Presented by F. Walker, Esq.

Entedon Acamas, Walker, Mon. Chal. i. 83.

a. England. Presented by F. Walker, Esq.

Entedon Lyœus, Walker, Mon. Chal. i. 84.

a. England. Presented by F. Walker, Esq.

Entedon Anthylla, Walker, Mon. Chal. i. 85.

a. England. Presented by F. Walker, Esq.

Entedon Loelius, Walker, Mon. Chal. i. 86.

a. England. Presented by F. Walker, Esq.

Entedon Clinius, Walker, Mon. Chal, i. 90.

a. England. Presented by F. Walker, Esq.

Entedon Rubigus, Walker, Mon. Chal. i. 91.

a. England. Presented by F. Walker, Esq.

Entedon Navius, Walker, Mon. Chal. i. 92.

a. England. Presented by F. Walker, Esq.

Entedon Diocles, Walker, Mon Chal. ii. 40.

a. Sydney, New South Wales. Presented by C. Darwin, Esq.

Entedon Alcœus, Walker, Mon. Chal. i. 94.

a. England. Presented by F. Walker, Esq.

b. Fontainbleau, France. Presented by the Entomological Club.

c. France. Presented by M. Brebisson.

d. Sweden. Presented by F. Walker, Esq.

Entedon Cleodora. Horismenus Cleodora, Walker, Ann. Nat. Hist. xi. 117.

a. Lima, Peru. Presented by C. Darwin, Esq.

Entedon Pronapis, Walker, Mon. Chal. ii. 40.

a. Sydney, New South Wales. Presented by C. Darwin, Esq.

Entedon Hestia. Eulophus Hestia, Walker, Mon. Chal. ii. 46. a. Hobart Town, Van Diemen's Land. Presented by C. Darwin, Esq.

Entedon Emperamus, Walker, Mon. Chal. ii. 70. a. Bahia, Brazil. Presented by F. Walker, Esq.

Entedon Hegelochus, Walker, Mon. Chal.ii. 70. a. Bahia, Brazil. Presented by C. Darwin, Esq. 
Entedon Antander, Walker, Mon. Chal. ii.70.

a. Bahia, Brazil. Presented by C. Darwin, Esq.

Entedon Thestius. Cirrospilus Thestius, Walker, Mon. Chal.

ii. 74.

a. Bahia, Brazil. Presented by C. Darwin, Esq.

5. Euderus, Haliday.

Euderus Amphis. Entedon Amphis, Walker, Mon. Chal. i. 106. a. England. Presented by F. Walker, Esq.

b. Alten, Finmark. Presented by F. Walker, Esq.

Euderus Mestor. Eulophus Mestor, Walker, Mon. Chal. ii. 42.

E. Capio, Walker, Mon. Chal. ii. 44.

a. King George's Sound, Australia. Presented by C. Darwin, Esq.

\section{Lopносомus, Haliday.}

Lopносомus Anaitis. Cirrospilus Anaitis, Walker, Mon. Chal. ii. 91.

a. Isle of Chiloe, Chili. Presented by C. Darwin, Esq.

\section{7. Орhецimus, Haliday.}

Ophelimus Ursidius, Haliday, Trans. Ent. Soc. iii. Eulophus Ursidius, Walker, Mon, Chal. ii. 44.

a. Hobart Town, Van Diemen's Land. Presented by C. Darwin, Esq.

Ophelinus Sabella. Eulophus Sabella, Walker, Mon. Chal. ii. 41. Cirrospilus Prymno, Walker, Mon. Chal. ii. 50.

a. Hobart Town, Van Diemen's Land. Presented by C. Darwin, Esq.

\section{Euplectrus, Westwood.}

Euplectrus bicolor. Pteromalus bicolor, Swederus, Kongl. Vet. Acad. Handl. 1795, 204, 2. Elachestus albiventris, Spinola, Ann. Mus. vii. 151. Nees. Hym. Ich. aff. Mon. ii. 146. Euplectrus Furnius, Walker, Ann. Nat. Hist. xii. 48. Euplectrus maculiventris, Westwood, Lond. and Edin. Phil. Mag. Third Series. i. 2.128. Eulophus bicolor, Walker, Mon. Chal. ii. 173, Spalangia? flavipes, Boyer de Fonscol. Ann. Sci. Nat. 1832, 299, 1.

a. England. Presented by F. Walker, Esq.

b. Sweden. Presented by F. W alker, Esq.

c. St. Vincent's Island, West Indies. Presented by the Entomological Club.

d. Hobart Town, Van Diemen's Land. Presented by C. Darwin, Esq. 


\section{Elachestus, Spinola.}

Elachestus dimidiatus. Eulophus dimidiatus, Nees. Hym. Ich. aff. Mon. ii. 160. Eulophus Artæus, Walker, Mon. Chal. i. 172.

a. England. Presented by F. Walker, Esq.

$b$. Sweden. Presented by F. Walker, Esq.

c. Fontainbleau, France. Presented by the Entomological Club.

Elachestus Charondas. Eulophus Charondas, Walker, Mon. Chal. i. 174.

a. England. Presented by F. Walker, Esq.

Elachestus Orsus. Eulophus Orsus, Walker, Mon. Chal. i. 174. a. England. Presented by F. Walker, Esq.

Elachestus Florianus. Eulophus Florianus, Walker, Mon. Chal. i. 176.

a. England. Presented by F. Walker, Esq.

Elachestus Eucrate. Eulophus Eucrate, Walker, Mon. Chal. i. 176.

a. England. Presented by F. Walker, Esq.

Elachestus Erse. Eulophus Erse, Walker, Mon. Chal. i. 177.

a. England. Presented by F. Walker, Esq.

$b$. South of France. Presented by the Entomological Club.

Elachestus Argissa, Walker, Mon. Chal. i. 172.

a. England. Presented by F. Walker, Esq.

$b$. South of France. Presented by the Entomological Club.

Elachestus Neleus. Eulophus Neleus, Walker, Mon. Chal.i. 177. a. England. Presented by F. Walker, Esq.

Elachestus Suada. Eulophus Suada, Walker, Mon. Chal. i. 178.

a. England. Presented by F. Walker, Esq.

Elachestus Amelon. Eulophus Amelon, Walker, Mon. Chal. i. 179

a. England. Presented by F. Walker, Esq.

Elachestus rufescens. Ichneumon rufescens, Rossi. Faun. Etr. Mant. App. ii. 113, 94. De Geer, Ins. ii. 189, 11. t. 30. f. 22. Vill. Ent. Lin. iii. 211, 248? Diplolepis rufescens, Spin. Ins. Lig. Fasc. iii. 160, 12 . Cleonymus rufescens, Lat. Gen. Crust. et Ins. iv. 29. Eulophus rufescens, Nees. Hym. Ich. aff. Mon. ii. 161. Stenomesius pulchellus Mas. Westwood, Lond. and Edin. Phil. Mag. iii. 343. St. maculatus, Fem. Westwood, Lond. and Edin. Phil. Mag. iii. $343 . \quad$ Cirrospilus Acesius, Walker, Ann. Nat. Hist.200. E. maculatus, Walker, Mon. Chal. i. 193.

a. England. Presented by F. Walker, Esq.

b. Sweden. Presented by F. Walker, Esq. 
Elachestus Gyes. Eulophus Gyes, Walker, Mon. Chal. ii. 89.

a. Isle of Chiloe, Chili. Presented by C. Darwin, Esq.

Elachestus Catta. Eulophus Catta, Walker, Mon. Chal. ii. 71. a. Bahia, Brazil. Presented by C. Darwin, Esq.

Elachestus. See Appendix. Eulophus Artœus, Walker, Mon. Chal. ii. 41.

a. Hobart Town, Van Diemen's Land. Presented by C. Darwin, Esq.

\section{Eulophus, Geoffroy.}

Eulophus ramicornis, Geof. Lat. Gen. Crustt et Ins. iv. 28. Diplolepis ramicornis, De Gen. ii. 313, pl. xv. f. 3. Fab. Syst. Piezat. 153. Eulophus damicornis, Kirb. Linn. Trans. xiv. 112.

a. England. Presented by F. Walker, Esq.

b. Sweden. Presented by F. Walker, Esq.

Eulophus Tyrrhenus, Walker, Mon. Chal. i. 147.

a. England. Presented by F. Walker, Esq.

$b$. Clermont, France. Presented by the Entomological Club.

Eulophus Laogonus, Walker, Mon. Chal. i. 151.

a. England. Presented by F. Walker, Esq.

Eulophus Sandanis, Walker, Mon. Chal. i. 130.

a. England. Presented by F. Walker, Esq.

Eccophus Rhianus, Walker, Ann. Nat. Hist. x. 116.

a. Valparaiso, Chili. Presented by C. Darwin, Esq.

Eulopнus Thespius, Walker, Mon. Chal. i. 127.

a. Sweden. Presented by F. Walker, Esq.

Eulophus Callidius, Walker, Mon. Chal. i. 144.

a. England. Presented by F. Walker, Esq.

Eulophus Cosconius, Walker, Mon. Chal. i. 145.

a. England. Presented by F. Walker, Esq.

Eulophus Faula, Walker, Mon. Chal. i. 148.

a. England. Presented by F. Walker, Esq.

Eulophus Iapetus, Walker, Mon. Chal. i. 144.

a. England. Presented by F. Walker, Esq.

Eulophus Hedila, Walker, Mon. Chal. i. 143.

a. England. Presented by F. Walker, Esq.

Eulophus Nycteus, Walker, Mon. Chal.i. 128.

a. England. Presented by F. Walker, Esq.

EuLophus Gonippus, Walker, Mon. Chal. i. 132.

a. England. Presented by F. Walker, Esq. 
Eu lophus Gordius, Walker, Mon. Chal. i. 129.

a. Sweden. Presented by F. Walker, Esq.

Eulophus Menyllus, Walker, Mon. Chal. i. 131.

a. England. Presented by. F. Walker, Esq.

Eulophus Myodes, Walker, Mon. Chal. i. 136.

a. England. Presented by F. Walker, Esq.

Eulophus Ianthea, Walker, Mon. Chal. i. 135.

a. England. Presented by F. Walker, Esq.

b. Fontainbleau, France. Presented by the Entomolosical ('lub.

Eulophus Dercynus, Walker, Mon. Chal. i. 139.

a. England. Presented by F. Walker, Esq.

Eu lophus Nonus, Walker, Mon. Chal. i. 142.

a. England. Presented by F. Walker, Esq.

Eulophus Hegemon, Walker, Mon. Chal. i. 140.

a. England. Presented by F. Walker, Esq.

Eulophus Bœotus, Walker, Mon. Chal. i. 151.

a. England. Presented by F. Walker, Esq.

Eulophus Trachalus, Walker, Mon. Chal. i. 152.

a. England. Presented by F. Walker, Esq.

Eulophus Dropion, Walker, Mon. Chal. i. 150.

a. England. Presented by F. Walker, Esq.

Eulophus Catreus, Walker, Mon. Chal. i. 148.

a. England. Presented by F. Walker, Esq.

Eulophus Itea, Walker, Mon. Chal. ii. 43.

a. Hobart Town, Van Diemen's Land. Presented by C. Darwin, Esq.

Eulophus Cicuta, Walker, Mon. Chal. ii. 43.

a. Sydney, New South Wales. Presented by C. Darwin, Esq.

Eu lophus Telestas, Walker, Mon. Chal. ii. 42.

a. Sydney, New South Wales. Presented by C. Darwin, Esq.

Eulophus Pisidice, Walker, Mon. Chal. i. 158.

a. England. Presented by F. Walker, Esq.

Eulophus Laodochus, Walker, Mon. Chal. i. 164.

a. Sweden. Presented by F. Walker, Esq.

Eulophus Sœmias, Walker, Mon. Chal. i. 170.

a. England. Presented by F. Walker, Esq.

Eulophus Lucumo, Walker, Mon. Chal. i. 169

a. England. Presented by F. Walker, Esq.

Eulophus Cromus, Walker, Mon. Chal. i. 165.

a. England. Presented by F. Walker, Esq. 
Eulophus Agraules, Walker, Mon. Chal. i. 166.

a. England. Presented by F. Walker, Esq.

Eulophus Artynes, Walker, Mon. Chal. i. 163.

a. England. Presented by F. Walker, Esq.

b. Sweden. Presented by F. Walker, Esq.

c. South of France. Presented by the Entomological Club.

Eulophus Alaparus, Walker, Mon. Chal. i. 163.

a. England. Presented by F. Walker, Esq.

b. Fontainbleau, France. Presented by the Entomological Club.

c. Sweden. Presented by F. Walker, Esq.

Eulophus Cœcilius, Walker, Mon. Chal. i. 166.

a. England. Presented by F. Walker, Esq,

b. Sweden. Presented by F. Walker, Esq.

Eulophus Faustitas, Walker, Mon. Chal. i. 167.

a. England. Presented by F. Walker, Esq.

Eulophus Mandron, Walker, Mon. Chal. i. 168.

a. England. Presented by F. Walker, Esq.

Eulophus Meriones, Walker, Mon Chal. i. 171.

a. England. Presented by F. Walker, Esq.

Eulophus Pisenor, Walker, Mon. Chal. i. 153.

a. England. Presented by F. Walker, Esq.

Eulophus Villius, Walker, Mon. Chal. i. 154.

a. England. Presented by F. Walker, Esq.

Eulophus fulvicollis, Walker, Mon. Chal. i. 190. Hemiptarsenus fulvicollis, Westwood, Loudon's Mag. Nat. Hist. vi. 123.

a. England. Presented by F. Walker, Esq.

Eulophus Waterhousii. Hemiptarsenus Waterhousii, Westwood, Mag. Nat. Hist. vi. 123.

a. England. Presented by G. R. Waterhouse, Esq.

Eulophus Anementus, Walker, Mon. Chal. i. 191.

a. England. Presented by F. Walker, Esq.

Eulophus Metalarus, Walker, Mon. Chal. i. 187.

a. England. Presented by F. Walker, Esq.

$b$. Clermont, France. Presented by the Entomological Club.

Eulophus Anıempsinus, Walker, Mon. Chal. i. 186.

a. England. Presented by F. Walker, Esq.

b. Clermont, France. Presented by the Entomological Club.

Eulophus Isadas, Walker, Mon. Chal. i. 168.

a. England. Presented by F. Walker, Esq.

Eulophus Rhœeus, Walker, Mon. Chal. i. 182.

a. England. Presented by F. Walker, Esq. 
Eulophus Hippia, Walker, Mon. Chal. i. 185.

a. England. Presented by F. Walker, Esq.

b. Sweden. Presented by F. Walker, Esq.

Eulophus Westwoodii, Mon. Chal. i. 157. Dicladocerus Westwoodii, Stephens, Westwood, Lond. and Edin. Phil. Mag. Third Series, i. 2, 128.

a. England. Presented by F. Walker, Esq.

b. Finmark. Presented by F, Walker, Esq.

c. Sweden. Presented by F. Walker, Esq.

Eulophus Battis, Walker, Mon. Chal. i. 162.

a. England. Presented by F. Walker, Esq.

Eulophus Eneugamus, Walker, Mon. Chal. i. 160.

a. England. Presented by F. Walker, Esq.

$b$. South of France. Presented by the Entomological Club.

Eucophus Euedoreschus, Walker, Mon. Chal. i. 188.

a. England. Presented by F. Walker, Esq.

b. Sweden. Presented by F. Walker, Esq.

Eur.ophus? Laonome, Walker, Mon. Chal. ii. 90.

a. Isle of Chiloe, Chili. Presented by C. Darwin, Esq.

\section{Belle rus, Haliday.}

Bellerus Anaitis, Haliday, MSS., Walker, Ann. Nat. Hist. xi. 32.

a. Concepcion, Chili. Presented by C. Darwin, Esq.

12. Cirrospilus, Westuood.

Cirrospilus vittatus, Walker, Ann. Nat. Hist. i. 308.

a. England. Presented by F. Walker, Esq.

Cirrospilus flavovarius. Eulophus flavovarius, Nees, Hym. Ich. aff. Mon.ii. 164. Cirr. Thasus, Walker, Ann. Nat. Hist.i.309.

a. England. Presented by F. Walker, Esq.

b. Sweden. Presented by F. Walker, Esq.

Cirrosprlus elegantissimus, 'Westwood, Lond. and Edin. Phil. Mag. i. 128.

a. England. Presented by F. Walker, Esq.

b. Sweden. Presented by F. Walker, Esq.

Cirrospilus Diallus, Walker, Ann. Nat. Hist. i. $31 \%$.

a. England. Presented by F. Walker, Esq.

Cirrospilus Salatis, Walker, Ann. Nat. Hist. i. 311.

a. England. Presented by F. Walker, Esq. 
Cirrospilus Singa, Walker, Ann. Nat. Hist. i. 383.

a. England. Presented by F. Walker, Esq.

Cirrospilus Crino, Walker, Ann. Nat. Hist. i. 382.

a. England. Presented by F. Walker, Esq.

Cirrospilus Lyncus, Walker, Ann. Nat. Hist. i. 381.

a. England. Presented by F. Walker, Esq.

Cirrospilus Buselus, Walker, Mon. Chal. ii. 96.

a. James' Island, Galapagos. Presented by C. Darwin, Esq.

Cirrospilus Isæa, Walker, Ann. Nat. Hist. i. 386. Eulophus leucarthros? Nees. Hym. Ich. aff. ii. 172.

a. England. Presented by F. Walker, Esq.

Cirrospilus Lycophron, Walker, Ann. Nat. Hist. i. 449. Var. Isæa?

a. England. Presented by F. Walker, Esq.

Cirrospilus Coronis, Walker, Ann. Nat. Hist. i. 450.

a. England. Presented by F. Walker, Esq.

Cirrospilus Clinias, Walker, Ann. Nat. Hist. i. 451.

a. England. Presented by F. Walker, Esq.

Cirrospilus Medidas, Walker, Ann. Nat. Hist. i. 386.

a. England. Presented by F. Walker, Esq.

Cirrospilus Minœus, Walker, Ann. Nat. Hist.i. 385. C. Abron,

Walker, Ann. Nat. Hist. i. 385. Eulophus Amelon, Walker,

Mon. Chal. i. 179.

a. England. Presented by F. Walker, Esq.

Cirrospilus Lycomedes, Walker, Ann. Nat. Hist. i. 450.

a. England. Presented by F. Walker, Esq.

Cirrospilus Chabrias, Walker, Ann. Nat. Hist. i. 451.

a. England. Presented by F. Walker, Esq.

Cirrospilus Acasta, Walker, Mon. Chal. i. 328.

a. England. Presented by F. Walker, Esq.

\section{Tetrastichus, Haliday.}

Tetrastichus Evonymellæ. Eulophus Evonymellæ, Bouché,

Naturges. der Ins. i. 172, 68.

a. England. Presented by F. Walker, Esq.

Tetrastichus Lagus. Cirrospilus Lagus, Walker, Ann. Nat.

Hist. i. 454.

a. England Presented by F. Walker, Esq.

Tetrastichus Articas. Cirrospilus Articas, Walker, Ann. Nat.

Hist. i. 199.

a. England. Presented by F. Walker, Esq. 
Tetrastichus Acesius. Cirrospilus Acesius, Walker, Ann. Nat, Hist. ii. 200.

a. England. Presented by F. Walker, Esq.

Tetrastichus Armœus. Cirrospilus Armœus, Walker, Ann. Nat Hist. ii. 200.

a. England. Presented by F. Walker, Esq.

Tetrastichus Mandanis. Cirrospilus Mandanis, Walker, Ann. Nat. Hist. ii. 202.

a. England. Presented by F. Walker, Esq.

Tetrastichus Eurytus. Cirrospilus Eurytus, Walker, Ann. Nat. Hist. ii. 202.

a. South of France. Presented by the Entomological Club.

Tetrastrchus Anysis. Cirrospilus Anysis, Walker, Ann. Nat. Hist. ii. 203.

a. England. Presented by F. Walker, Esq.

Terrastichus Zeuxo. Cirrospilus Zeuxo, Walker, Mon. Chal. i. 194 .

a. South of France. Presented by the Entomological Club.

Tetrastichus Ecus. Cirrospilus Ecus, Walker, Ann. Nat. Hist. ii. 204.

a. England. Presented by F. Walker, Esq.

Tetrastichus Attalus. Cirrospilus Attalus, Walker, Ann. Nat. Hist. ii. 353.

a. England. Presented by F. Walker, Esq.

b. Sweden. Presented by F. Walker, Esq.

Tetrastichus Adalia. Cirrospilus Adalia, Walker, Ann. Nat. Hist. ii. 351.

a. England. Presented by F. Walker, Esq.

Tetrastichus Orithyia. Cirrospilus Orithyia, Walker, Ann. Nat. Hist. ii. 352.

a. England. Presented by F. Walker, Esq.

Tetrastrchus Agathocles. Cirrospilus Agathocles, Walker, Ann.

Nat. Hist. ii. 353.

a. Eugland. Presented by F. Walker, Esq.

Tetrasticnus Idithyia. Cirrospilus Idithyia, Walker, Ann. Nat. Hist. ii. 135.

a. England. Presented by F. Walker, Esq.

Tetrastichus Molo. Cirrospilus Molo, Walker, Ann. Nat. Hist. iii. 179.

a. England. Presented by F. Walker Esq.

Tetrastichus Epicharmus. Cirrospilus Epicharmus, Walker, Ann. Nat. Hist. iii. 180.

a. England. Presented by F. Walker, Esq. 
Tetrastichus Aristœus. Cirrospilus Aristœus, Walker, Ann. Nat. Hist. iii. 416.

a. England. Presented by F. Walker, Esq.

Tetrastichus Rhipheus. Cirrospilus Rhipheus, Walker, Ann. Nat. Hist. iii. 416.

a. England. Presented by F. Walker, Esq.

Tetrastichus Menius. Cirrospilus Menius, Walker, Ann. Nal. Hist. iii. 179.

a. England. Presented by F. Walker, Esq.

Tetrastichus Endemus. Cirrospilus Endemus, Walker, Anr. Nat. Hist. iii. 180.

a. England. Presented by F. Walker, Esq.

Tetrastichus Achæmenes. Cirrospilus Achæmenes, Walker, Ann. Nat. Hist. iii. 419.

a. England. Presented by F. Walker, Esq.

Texrastichus Lycippe. Cirrospilus Lycippe, Walker, Ann. Nut. Hist. iii. 419.

a. England. Presented by F. Walker, Esq.

Tetrastrchus Charoba. Cirrospilus Charoba, Walker, Ann. Nat. Hist. iv. 30.

a. England. Presented by F. Walker, Esq.

Tetrastichus Zosimus. Cirrospilus Zosimus, Walker, Mon. Chal. i. 297.

a. England. Presented by F. Walker, Esq.

Tetrastichus Upis. Cirrospilus Upis, Walker, Mon. Chal. i. 297.

a. England. Presented by F. Walker, Esq.

b. Clermont, France. Presented by the Entomological Club.

Tetrastichus Monesus. Cirrospilus Monesus, Walker, Mon. Chal. i. 297.

a. England. Presented by F. Walker, Esq.

Temrastichus Xeuxes. Cirrospilus Xeuxes, Walker, Mon. Chal. i. 301 .

a. England. Presented by F. Walker, Esq.

Tetrastichus Prosymna. Cirrospilus Prosymna, Walker, Mon. Chal. i. 304.

a. England. Presented by F. Walker, Esq.

b. Fontainbleau, France. Presented by the Entomological Club.

a. South of France. Presented by the Entomological Club.

Tetrastichys Xixuthrus. Cirrospilus Xixuthrus, Walker, Mon. Chal. i. 306.

a. England. Presented by F. Walker, Esq. 
Tetrastichus Quercens. Cirrospilus Quercens, Walker, Mon. Chal. i. 307.

a. England. Presented by F. Walker, Esq.

Tetrastichus Ligus. Cirrospilus Ligus, Walker, Mon. Chal. i. 300.

a. England Presented by F. Walker, Esq.

Tetrastichus Lacæna. Cirrospilus Lacæna, Walker, Mon. Chal. i. 302 .

a. England. Presented by F. Walker, Esq.

Tetrastichus Orodes. Cirrospilus Orodes, Walker, M̀on. Chal. i. 303 .

a. England. Presented by F. Walker, Esq.

Tetrastichus Orsedice. Cirrospilus Orsedice, Walker, Mon. Chal. i. 308.

a. England. Presented by F. Walker, Esq.

Tetrastichus Tenerus. Cirrospilus Tenerus, Walker, Mon. Chal. i. 308 .

a. England. Presented by F. Walker, Esq.

Tetrastichus Sucro. Cirrospilus Sucro, Walker, Mon. Chal. i. 308.

a. England. Presented by F. Walker, Esq.

Tetrastichus Sandace. Cirrospilus Sandace, Waller, Mon. Chal. i. 311.

a. England. Presented by F. Walker, Esq.

Tetrastichus Novatus. Cirrospilus Novatus, Walker, Mon. Chal. i. 312.

a. England. Presented by F. Walker, Esq.

Tetrastichus Athyrte. Cirrospilus Athyrte, Walker, Ann. Nat. Hist. iii. 178.

a. England. Presented by F. Walker, Esq.

Tetrastichus Bunus. Cirrospilus Bunus, Walker, Ann. Nat. Hist. iii. 179.

a. England. Presented by F. Walker, Esq.

Tetrastichus Rhacius. Cirrospilus Rhacius, Walker, Ann. Nat. Hist. iii. 181.

a. England. Presented by F. Walker, Esq.

Tetrastichus Sotades. Cirrospilus Sotades, Walker, Ann. Nat. Hist. iii. 417.

a. England. Presented by F. Walker, Esq.

Tetrastichus Rapo. Cirrospilus Rapo, Walker, Ann. Nat. Hist. iii. 415.

a. England. Presented by F. Walker, Esq. 
Tetrastichus Nerio. Cirrospilus Nerio, Walker, Mon. Chal. i. 295.

a. England. Presented by F. Walker, Esq.

b. Sweden. Presented by F. Walker, Esq.

Tetrastichus Paralus. Cirrospilus Paralus, Walker, Mon. Chal. i. 296.

a. England. Presented by F. Walker, Esq.

Tetrastichus Tymber. Cirrospilus Tymber, Walker, Mon. Chal. i. 298.

a. England. Presented by F. Walker, Esq.

Tetrastichus Nymphis. Cirrospilus Nymphis, Walker, Mon. Chal. i. 299.

a. England. Presented by F. Walker, Esq.

Tetrastichus Deipyrus. Cirrospilus Deipyrus, Walker, Mon. Chal. i. 299.

a. England. Presented by F. Walker, Esq.

Tetrastichus Eupolis. Cirrospilus Eupolis, Walker, Mon. Chal. i. 302.

a. England. Presented by F. Walker, Esq.

Tetrastichus Phineus. Cirrospilus Phineus, Walker, Mon. Chal. i. 303.

a. England. Presented by F. Walker, Esq.

b. Fontainbleau, France. Presented by the Entomological Club.

c. South of France. Presented by the Entomological Club.

Tetrastichus Mazœus. Cirrospilus Mazœus, Walker. More. Chal. i. 302.

a. England. Presented by F. Walker, Esq.

Tetrastichus Hippis. Cirrospilus Hippis, Walker, Mon. Chal. i. 304 .

a. England. Presented by F. Walker, Esq.

Tetrastichus Rabirius. Cirrospilus Rabirius, Walker, Mon. Chal. i. 305.

a. England. Presented by F. Walker, Esq.

Tetrastrchus Asopus. Cirrospilus Asopus, Walker, Mon. Chal. i. 314 .

a. England. Presented by F. Walker, Esq.

Tetrastichus Pronomus. Cirrospilus Pronomus, Walker, Mon.

Chal. i. 312. Pteroptrix Actis, Walker, Mon. Chal. i. 20.

a. England. Presented by F. Walker, Esq.

Tetrastichus Amynus. Cirrospilus Amynus, Walker, Mon.

Chal. i. 313.

a. England. Presented by F. Walker, Esq. 
Tetrastichus Amenon. Cirrospilus Amenon, Walker, Mon. Chal. i, 315.

a. England. Presented by F. Walker, Esq.

Tetrastichus Deioces. Cirrospilus Deioces, Walker, Mon. Chal. i. 315 .

a. England. Presented by F. Walker, Esq.

Tetrastichus Diaphantus. Cirrospilus Diaphantus, Walker, Mon. Chal. i. 327.

a. England. Presented by F. Walker, Esq.

Tetrastichus Isander. Cirrospilus Isander, Walker, Mon. Chal. i. 326.

a. England. Presented by F. Walker, Esq.

Tetrastichus Lamius. Cirrospilus Lamius, Walker, Mon. Chal. i. 327.

a. England. Presented by F. Walker, Esq.

Tetrastichus Rhœo. Cirrospilus Rhœo, Walker, Ann. Nat. Hist. iii. 417.

a. England. Presented by F. Walker, Esq.

Tetrastichus Alcithoe. Cirrospilus Alcithoe, Walker, Ann. Nat. Hist. iii. 418.

a. England. Presented by F. Walker, Esq.

Tetrastichus Phalis. Cirrospilus Phalis, Walker, Ann. Nat. Hist. iii. 418.

a. England. Presented by F. Walker, Esq.

Tetrastichus Catius. Cirrospilus Catius, Walker, Mon. Chal. i. 323.

a. England. Presented by F. Walker, Esq.

Tetrastichus Anteius. Cirrospilus Anteius, Walker, Mon. Chal. i. 324. Eulophus ater? Nees, ab Ess. Hym. Ich. aff. Mon. ii. 186.

a. England. Presented by F. Walker, Esq.

Tetrastichus caudatus. Aprostocetus caudatus, Westwood, Lond. and Edin. Phil. Mag. ii. 444. Cirrospilus caudatus, Walker, Mon. Chal. i. 324. Eulophus glabellus? Nees. ab. Ess. Hym. Ich.aff. Mon. ii. 186.

a. England. Presented by F. Walker, Esq.

Tetrastichus Emesa. Cirrospilus Emesa, Walker, Mon. Chal. i. 324 .

a. England. Presented by F. Walker, Esq.

Tetrastichus Zoilus. Cirrospilus Zoilus, Walker, Mon. Chal, i. 325 .

a. England. Presented by F. Walker, Esq. 
Tetrastichus Leucone. Cirrospilus Leucone, Walker, Mon. Chal. i. 325.

a. England. Presented by F. Walker, Esq.

b. Clermont, France. Presented by the Entomological Club.

Tetrastichus Daira. Cirrospilus Daira, Walker, Mon. Chal. i. 330.

a. England. Presented by F. Walker, Esq.

Tetrastichus Orsillus. Cirrospilus Orsillus, Walker, Mon. Chal. i. 318 .

a. England. Presented by F. Walker, Esq.

Tetrastichus Xenares. Cirrospilus Xenares, Walker, Mon. Chal. ii. 54.

a. Hobart Town, Van Diemen's Land. Presented by C. Darwin, Esq.

b. King George's Sound, Australia. Presented by C. Darwin, Esq.

Tetrastichus Arses. Cirropilus Arses, Walker, Mon. Chal. ii. 55.

a. Hobart Town, Van Diemen's Land. Presented by C. Darwin, Esq.

b. King George's Sound, Australia. Presented by C. Darwin, Esq.

Tetrastichus Hippasus. Cirrospilus Hippasus, Walker, Mon. Chal. ii. 51.

a. Hobart Town, Van Diemen's Land. Presented by C. Darwin, Esq.

Tetrastichus Lelaps. Cirrospilus Lelaps, Walker. Mon. Chal. ii. 51.

a. King George's Sound, Australia. Presented by C. Darwin, Esq.

Tetrastichus Fannius. Cirrospilus Fannius, Walker, Mon. Chal. ii. 49.

a. Hobart Town, Van Diemen's Land. Presented by C. Darwin, Esq.

Tetrastichus Zaleucus, Cirrospilus Zaleucus, Walker, Mon. Chal. ii. 52.

a. Hobart Town, Van Diemen's Land. Presented by C. Darwin, Esq.

Terrastichus Valens. Cirrospilus Valens, Walker, Mon. Chal. ii. 46.

a. Hobart Town, Van Diemen's Land. Presented by C. Darwin, Esq.

Tetrastichus Dymas. Eulophus Dymas, Walker, Mon. Chal. ii. 45. 
a. Hobart Town, Van Diemen's Land. Presented by C. Darwin, Esq.

Tetrastichus Otys. Cirrospilus Otys, Walker. Mon. Chal. ii. 48.

a. Sydney, New South Wales. Presented by C. Darwin, Esq.

Tetrastichus Proto. Cirrospilus Proto, Walker, Mon. Chal. ii. 47.

a. Hobart Town, Van Diemen's Land. Presented by C. Darwin, Esq.

Tetrastichus Baucis. Cirrospilus Baucis, Walker, Mon. Chal. ii. 53.

a. King George's Sound, Australia. Presented by C. Darwin, Esq.

Tetrastichus Glycon. Cirrospilus Glycon, Walker, Mon. Chal. ii. 54 .

a. Hobart Town, Vau Diemen's Land. Presented by C. Darwin, Esq.

Tetrastichus Autonæ. Eulophus Autonæ, Walker, Mon. Chal. ii. 45.

a. Hobart Town, Van Diemen's Land. Presented by C. Darwin, Esq.

Tetrastichus Neis. Cirrospilus Neis, Walker, Mon. Chal. ii. 49.

a. Hobart Town, Van Diemen's Land. Presented by C. Darwin, Esq.

Tetrastichus granulatus, Barnstone, MSS., Walker, Ann. Nat. Hist. xiv. 17.

a. Martin's Falls, Albany River, Hudson's Bay. Presented by G. Barnstone, Esq.

Tetrastichus Daimachus. Cirrospilus Daimachus, Walker, Mon. Chal. ii. 73.

a. Bahia, Brazil. Presented by C. Darwin, Esq.

Tetrastichus Februus. Cirrospilus Februus, Walker, Mon. Chal. ii. 73.

a. Bahia, Brazil. Presented by C. Darwin, Esq.

Tetrastichus Valerus. Cirrospilus Valerus, Walker, Mon. Chal. ii. 72.

a. Bahia, Brazil. Presented by C. Darwin, Esq.

Tetrastichus Athenais. Cirrospilus Athenais, Walker, Mon. Chal. ii. 72.

a. Bahia, Brazil. Presented by C. Darwin, Esq.

Tetrastichus Cacus. Cirrospilus Cacus, Walker, Mon. Chal. ii. 75.

a. Bahia, Brazil. Presented by C. Darwin, Esq. 
Tetrastichus Archideus. Cirrospilus Archideus, Walker, Mon.

Chal. ii. 75.

a. Bahia, Braxil. Presented by C. Darwin, Esq.

Tetrastichus Deilochus. Cirrospilus Deilochus, Walker, Mon. Chal. ii. 74.

a. Bahia, Brazil. Presented by C. Darwin, Esq.

Tetrastichus Cleonica. Cirrospilus Cleonica, Walker, Mon. Chal. ii. 76.

a. Bahia, Brazil. Presented by C. Darwin, Esq.

Tetrastichus Xenocles. Cirrospilus Xenocles, Walker, Mon. Chal. ii. 90.

a. Island of Chiloe, Chili. Presented by C. Darwin, Esq.

Tetrastichus Scadius, Walker, Ann. Nat. Hist. x. 116.

a. Valparaiso, Chili. Presented by C. Darwin, Esq.

Tetrastichus Polybæa, Walker, Ann. Nat. Hist. x. 116.

a. Valparaiso, Chili. Presented hy C. Darwin, Esq.

Tetrastichus Naueles, Walker, Ann. Nat. Hist. xi. 32.

a. Concepcion, Chili. Presented by C. Darwin, Esq.

Tetrastichus Norax, Walker, Ann. Nat. Hist. xi. 32.

a. Concepcion, Chili. Presented by C. Darwin, Esq.

Tetrastichus Narcœus, Walker, Ann. Nat. Hist. xi. 188.

a. Coquimbo, Chili. Presented by C. Darwin, Esq. 



\section{APPENDIX.}

Page 5. Chalcis. Chalcis Visellus, Walker, Ann. Nat. Hist. February, 1846. Chalcis Amphilochus, Walker, Ann. Nat. Hist., February 1846.

Page 5. Сhalcis Amphissa, Walker, MSS. Nigra, pedibus luteis, coxis femoribusque nigris, his apice luteis, tibiis nigrovittatis, alis limpidis.

Black, dull, punctured : antennæ black : scutellum with a rim along its hind border : abdomen smooth, shining: legs luteous; coxæ and thighs black, tips of the latter luteous; a black stripe along each tibia: wings limpid; squamulæ yellow; nervures piceous. Length of the body $2 \frac{1}{2}$ lines; expansion of the wings 5 lines.

a. Nepal. From the Hardwicke bequest.

Page 5. Снацcis Xerxena, Walker, MSS. Nigra, antennis piceis basi rufis, pedibus piceo-rufis, alis limpidis.

Black, dull, punctured : antennæ dark piceous ; first joint dull red: hind-border of the scutellum armed with two very short teeth: abdomen smooth, shining, rather longer than the thorax: legs dull-red, slightly piceous: wings limpid; squamulæ brown; nervures piceous. Length of the body 2 lines; expansion of the wings 3 lines.

Page 5. Chalcis Alexion, Walker, MSS. Nigra, metafemoribus rufis, metatibiis piceis rufo variis, alis subfuscis.

Body black, dull, punctured: antennæ black: scutellum slightly furrowed; its hind border armed with two very short 
teeth; propodeon rugulose, slightly spined on each side : abdomen shining, finely punctured, pubescent towards the tip : legs black; hind thighs red; hind tibiæ dark piceous, varied with red : wings slightly fuscous; squamulæ black; nervures dark piceous. Length of the body $2 \frac{1}{4}$ lines; expansion of the wings 5 lines.

a. Italy. From Mr. Birch's collection.

CHalcıs Almon. Nigra, trochanteribus, genubus, tibiis apice tarsisque flavis, alis limpidis.

Body convex, black : head and thorax dull, punctured, pubescent : antennæ black: scutellum with a rim along its hind border : abdomen oval, smooth, shining, pubescent towards the tip, shorter and narrower than the thorax: legs black; trochanters, knees, tips of the tibiæ and tarsi yellow: wings limpid; squamulæ yellow; nervures fuscous. Length of the body $1 \frac{1}{2}$ line; expansion of the wings 2 lines.

a. Isle of France.

Chalcis Amenocles, Walker, MSS. Nigra, pedibus rufis, coxis nigris, femoribus apice tibiisque albis, his rufo cinctis, alis limpidis.

Black, dull, punctured: hind border of the scutellum armed with two very short teeth: abdomen smooth, shining, rather longer than the thorax : legs red; coxæ black ; tips of thighs white; tibiæ white, each traversed by a broad red band: wings limpid; squamulæ white; nervures black. Length of the body 3 lines; expansion of the wings 4 lines.

a. Sierra Leone.

Chalcis Ucalegon, Walker, MSS. Nigra, untennis pedibusque rufis, profemoribus et mesofemoribus nigris apice albis, metafemoribus apice albis, subtus dentibus nigris armatis, alis limpidis.

Body convex: head and thorax black, roughly punctured: antennæ bright pale red; first and second joints black: tip of the scutellum bituberculate : propodeon roughly excavated abdomen wanting: legs red; fore-thighs and middle thighs black, their tips white, as are also those of the hind-thighs, which are armed beneath with many little black teeth: wings limpid; nervures piceous; humerus more than twice the length of the ulna; radius and cubitus very short; stigma very small. Length of the body $2 \frac{1}{2}$ lines; expansion of the wings $3 \frac{1}{2}$ lines.

a. Adelaide, Australia. Presented by the Entomological Club.

Page 7. Haltichella Mitys, Walker, MSS. Nigra abdomine apice rufo, antennis pedibusque ferrugineis, femoribus subfuscis, alis limpidis fusco maculatis. 
Body convex, finely punctured, slightly shining: antennæ ferruginous, nearly filiform, as long as the thorax; first joint slightly dilated at the tip: scutellum slightly furrowed, armed with two very short teeth at its tip: metapodeon slightly carinated: podeon very short: abdomen smooth, shining, as long as the thorax, red towards the tip which is somewhat attenuated: legs ferruginous; thighs slightly fuscous: wings nearly limpid; a large fuscous spot beneath the stigma of each fore-wing; nervures piceous; ulna, radius, and cubitus very short ; stigma very small. Length of the body 2 lines; expansion of the wings 3 lines.

a. Isle of France.

\section{Page 7. Haltichella Amage.-H. Proxenus. Page 7.}

Page 7. Dirhinds Anthracia, Walker, MSS. Nigra, pedibus rufis, metapedibus nigris, alis limpidis.

Body black, dull, punctured : antennæ black: head clothed beneath with silvery down: tip of the scutellum dentate: abdomen smooth, shining, much shorter than the thorax: legs red; hind legs black : wings limpid; squamulæ and nerrures piceous. Length of the body 2 lines; expansion of the wings $2 \frac{1}{2}$ lines.

Page 8. Eurytoma fumipennis, Walker, Ent. Mag. iv. 24.

a. England. Presented by F. Walker, Esq.

Page 10. Eurytoma Iphis, Atra, genubus tarsisque mufis, his apice piceis, alis subfuscis.

Female; Body convex, black; head and thorax roughly punctured, sparingly clothed with white hairs : head broader than the thorax; antennæ clavate, much shorter than the thorax; first joint long, slender ; second cyathiform ; third and fourth very minute; fifth and following joints to the ninth nearly cyathiform, successively shorter and broader; club long, conical, near thrice the length of the ninth joint, its joints not being more closely fitted to each other than are the preceding joints : thorax long obconical: prothorax large, subquadrate, not narrower than the mesothorax; its breadth rather more than twice its length : scutum of the mesothorax short, broad; sutures of the parapsides distinct, approaching each other ; axillæ large triangular, not decumbent ; scutellum nearly rhomboidal: metathorax or propodeon large, transverse, decumbent : podeon very short: abdomen elliptical, smooth, shining, shorter and a little narrower than the thorax; its length a little more than twice its height; metapodeon conical, decumbent in front, less than one fourth of the length of the abdomen; octoon short, less than half the length of the metapodeon; ennaton much longer than the octoon; decaton as long as the ennaton; protelum, 
paratelum and telum very short; oviduct enclosed in a sheath which only just passes the tips of the abdomen: legs slender; knees and tarsi red, tip of the latter piceous: wings slightly fuscous; nervures piceous; humerus much less than half the length of the wing; ulna thick, hardly more than one third of the length of the humerus; radius a little shorter than the ulna ; cubitus much shorter than the radius; stigma very small, emitting a short branch.

Page 21. Eucharis Eginetus, Walker, MSS. Viridis abdomine rufo, antennis piceis 9-ramosis, pedibus fulvis, coxis viridibus, alis fusco nebulosis.

Male; Head and thorax convex, punctured, bright green : head transverse, short ; vertex broad ; front large, abruptly decumbent: mouth fulvous : eyes piceous, oval, of moderate size : antennæ filiform, slender, piceous, pubescent, adorned with nine branches, rather longer than the head and thorax ; first joint fulvous, long ; the following joints long, those towards the tip longer : thorax oval: prothorax extremely short, not visible above: scutum of the mesothorax broad; sutures of the parapsides indistinct; scutellum obconical, abruptly decumbent beyond the tip which has no spines: propodeon large, obconical, abruptly decumbent : podeon green, cylindrical, very slender, as long as the abdomen : abdomen fusiform, red, smooth, shining, fusiform, slightly compressed, narrower and rat.ler shorter than the thorax; metapodeon very large, occupying all the dorsum, and hiding the ventral segments : legs very slender, fulvous, pubescent; coxæ small, green; spines of the tibiæ small; first joint of the tarsi nearly as long as the other four: wings limpid; fore-wings slightly clouded with fuscous, each having a large fuscous spot beneath the stigma; nervures piceous; humerus much longer than the ulna; radius rather shorter than the ulna; cubitus extremely short; stigma small. Length of the body $2 \frac{1}{2}$ lines; expansion of the wings $4 \frac{1}{2}$ lines.

Page 21. Eucharis Larymna, Walker, MSS. Cuprea, abdomine rufo, antennis nigris, pedibus rufis, coxis cupreis, alis subfuscis.

Male and Female; Head and thorax transversely rugulose, convex, bright cupreous, varied with bright green : eyes black, of moderate size: mouth pale red: antennæ black, filiform, pubescent: antennæ of the male nearly as long as the body; joints long and linear: antennæ of the female shorter: thorax slightly gibbous: prothorax extremely short, not visible above : scutum of the mesothorax large, transverse; sutures of the parapsides indistinct, converging towards the scutellum; axillæ remote ; scutellum 
large, obconic above, abruptly decumbent at the tip ; metathorax very short: propodeon large, obconic, declining, roughly punctured: podeon dark purple, extremely long and slender: abdomen elliptical, slightly compressed, smooth, shining, dull red, shorter than the podeon, much shorter and smaller than the thorax ; metapodeon extremely large, occupying the whole of the dorsum and almost hiding the ventral segments: legs pubescent, red, very slender; coxæ small, cupreous, rugulose; spines of the tibiæ small, black; first joint of the tarsi very long, equal in length to the other four joints; claws and pulvilli small: wings very slightly fuscous; nervures piceous; humerus much longer than the ulna; radius much shorter than the ulna; cubitus extremely short. Length of the body 4 lines; expansion of the wings 6 lines.

Page 21. Eucharis Pyttalus, Walker, MSS. Fem. Viridis, abdominis disco cupreo, antennis piceis, pedibus flavis, alis limpidis. (Corp. long. lin. $1 \frac{1}{2}$; alar. lin. 3.)

Body convex, bright-green, here and there æneous : head and thorax rugulose: head transverse, very short, tuberculate, as broad as the thorax: eyes small, round, prominent: ocelli near together on the vertex, the middle one a little in advance of the other two: mandibles fulvous, long, arcuate, armed with long sharp teeth, two on one mandible and one on the other : antennæ piceous, filiform, a little shorter than the thorax ; first joint long, slender, yellow; second cyathiform; third and fourth extremely minute; fifth and following joints to the twelfth successively decreasing in length; thirteenth joint somewhat fusiform, slender towards the tip, nearly twice the length of the twelfth joint : thorax elliptical : prothorax extremely short, hardly visible above, transversely striated: scutum of the mesothorax of moderate size, rather broader than long, very slightly furrowed along the disk; sutures of the parapsides very distinct, approaching each other; axillæ large, triangular, almost conniving, approaching much nearer to each other than in the Eurytomidæ and the Torymidæ; scutellum subrhomboidal, rather large, declining behind: metathorax transverse, very short: propodeon large, obconical, decumbent, having a rim on each side : podeon short, stout: abdomen conical, minutely squameous, concave above, keeled beneath, cupreous on the disk, nearly as broad as the thorax but much shorter; metapodeon occupying all the dorsum : legs yellow, slender; coxæ blue, scaly; joints of the tarsi from the first to the fourth successively decreasing in length ; fifth joint longer than the fourth: wings ample, limpid; nervures fuscous; humerus about one-third of the length of the wing; ulna fully as long as the humerus; radius extending to the tip of the wing, nearly 
as long as the ulna; cubitus very short, not one-sixth of the length of the radius; stigma rather large, emitting no branch.

'Thoracantha Anchurus, Walker, MSS. Nigrocyanea antennis nigris, basi fulvis, pedibus flavis, alis limpidis.

Body bluish-black, gibbous, shining: head and thorax transversely rugulose: head not so broad as the thorax; front smooth, shining: mouth fulvous: antennæ black; first joint fulvous: scutellum longitudinally furrowed, and armed with two striated very long and slightly bent spines: propodeon very large, rugulose, slightly furrowed: podeon cylindrical, very slender, as long as the propodeon : abdomen compressed, smooth, shining, shorter and narrower than the thorax; metapodeon hiding all the other segments : legs yellow, very slender ; coxæ black ; wings limpid; nervures pale-yellow; humerus nearly twice the length of the ulna; radius none; cubitus very short; stigma extremely small. Length of the body 2 lines; expansion of the wings 4 lines.

a. Brazil.

Page 22. Thorocantha Nasua, Walker, MSS. Viridis cupreo et cyaneo varia, antennis ferrugineis, 8-ramosis, basi fulvis, pedibus fulvis, coxis cyaneoviridibus, alis limpidis, proalis fusco subnebulosis.

Male; Body convex, bright green, slightly varied with blue and cupreous: head transverse, short; vertex broad, minutely punctured; front large, almost smooth, abruptly decumbent: mouth fulvous : eyes piceous, oval, of moderate size : antennæ filiform, ferruginous, pubescent, adorned with eight branches, as long as the head and thorax ; first joint fulvous, long and stout; the fcllowing joints very short; those towards the tip longer: thorax gibbous, coarsely punctured: prothorax extremely short, not visible above: scutum of the mesothorax large; sutures of the parapsides distinct, approximating near together on the foreborder of the scutellum; scutellum two-lobed ; the first lobe transverse, narrow ; the second prominent, obconical, abruptly decum. bent behind, furrowed lengthwise above, the tip divided into two long spines which are slightly curved inwards : metathorax very short : propodeon large, obconic, abruptly declining : podeon stout, nearly smooth, less than half the length of the abdomen : abdomen oval, bluish-green, smooth, shining, slightly compressed, much shorter and narrower than the thorax; metapodeon extremely large, occupying all the dorsum, and hiding the ventral segments: legs very slender, fulvous, pubescent; coxæ small, bluish-green; spines of the tibiæ small; first joint of the tarsi nearly as long as the other four; third joint shorter than the second, but longer than the fourth; fifth joint nearly as long as 
the second: wings limpid ; fore-wings slightly clouded with fuscous; nervures piceous; humerus very much longer than the ulna which is thick at the tip; radius shorter than the ulna; cubitus very short. Length of the body 2 lines; expansion of the wings 4 lines.

Page 22. Perilampus Alexinus, Walker, MSS. Nigroæneus, angustus, antennis piceis, pedibus fulvis piceo variis, alis limpidis.

Eneous black, convex, coarsely punctured, more slender than is usual in this genus: antennæ piceous: tip of the scutellum armed with two teeth: propodeon rugulose : abdomen oval, smooth, shining, thinly clothed with hairs, much shorter than the thorax; metapodeon occupying more than half the dorsum; octoon, ennaton and decaton short; protelum and following segments very short: ventral segments not hidden by those of the dorsum; metapodeon large; the following segments short and successively decreasing in length: legs fulvous; coxæ and thighs piceous, tips of the latter fulvous; tibiæ irregularly encircled with fuivous: wings limpid; squamulæ yellow; nervures fulvous; humerus thrice the length of the ulna; radius and cubitus extremely short; stigma very small. Length of the body $2 \frac{1}{2}$ lines; expansion of the wings 4 lines.

Perilampus Lepreos, Walker, MSS. Nigroaneus, latus, antennis pedibusque piceis, tarsis fulvis, alis limpidis.

Eneous black, gibbous, neatly punctured, broader than the preceding species: head almost smooth: antennæ piceous: scutellum prominent, having a rim along its hind border whence it is abruptly decumbent : propodeon almost smooth with a longitudinal furrow: abdomen smooth, shining, broader than long, not half the length of the thorax; metapodeon occupying the whole of the dorsum.: legs piceous; knees and tarsi fulvous: wings limpid, squamulæ and nervures fulvous. Length of the body $1 \frac{1}{2}$ line; expansion of the wings 3 lines.

a. Georgia. From Mr. Abbot's collection.

Page 30. Lamprotatus Babilus, Walker, Ann. Nat. Hist. February, 1846.

Body stout, gibbous, shining, bluish-green : head and thorax finely squameous: head a little broader than the thorax : antennæ black, subclavate, not longer than the thorax; the joints from the fifth to the tenth successively decreasing in length; club conical : prothorax of moderate size: scutum transverse; sutures of the parapsides very distinct; scutellum prominent, with a trans- 
verse suture near its tip: propodeon large, obconic, declining, not furrowed: podeon stout, moderately long: abdomen smooth, keeled beneath, thinly clothed with white hairs, shorter than the thorax ; metapodeon deeply excavated; octoon, ennaton and decaton rather large; the rest very short: legs piceous ; coxæ and thighs green, knees and base of tibiæ fulvous : wings fuscous; nervures piceous; humerus more than twice the length of the ulna; radius much longer than the ulna; cubitus nearly as long as the ulna; stigma rather large. Length of the body $1 \frac{1}{4}$ line; expansion of the wings $2 \frac{1}{2}$ lines.

Page 30. Lamprotatus chrysochlorus, Haliday. Aneoviridis, antennis nigris, pedibus flavis fusco vuriis, alis limpidis.

Male; Body convex : head and thorax squameous : head a little broader than the thorax: antennæ slender, filiform, rather longer than the thorax; first joint long, slightly dilated beneath; second nearly round; third and fourth very minute; fifth and following joints to the tenth long, linear, successively decreasing in length ; club linear, very long, acuminate, thrice the length of the tenth joint: thorax elliptical: prothorax transverse, short, rather small, narrow in front: scutum of the mesothorax large, having a distinct channel across the middle; sutures of the parapsides distinct, approaching each other; axillæ large, triangular, not conniving; scutellum conical, not impressed in front, but having a transverse channel near the tip: metathorax large, obconical, decumbent: podeon siout, very short, much less thau the length of one-sixth of the abdomen: abdomen fusiform, depressed, very finely squameous, rather shorter and narrower than the thorax; metapodeon large, conical, smooth, covering nearly one half of the dorsum; octoon shorter than one-fourth of the metapodeon; ennaton more than twice the length of the octoon; decaton as long as the octoon; protelum, paratelum, and telum very short: legs slender: wings ample; humerus less than one-half the length of the wing; ulna about half the length of the humerus; radius very much longer than the ulna; cubitus hardly more than half the length of the ulna ; stigma rather large, pyriform, emitting a short branch towards the radius.

Female; Antennæ slender, filiform, as long as the thorax; first joint long, slender; second cyathiform; third and fourth very minute; fifth and following joints to the tenth long, linear, successively decreasing in length; club linear, acuminate, twice the length of the tenth joint: scutum of the mesothorax not channelled: abdomen smooth, oval, acuminated, depressed above, deeply keeled beneath, shorter but hardly narrower than the thorax; metapodeon large, conical, occupying nearly one-half of the dorsum; octoon, ennaton, and decaton equal in size, each rather 
shorter than one-third of the metapodeon ; protelum shorter than the metapodeon; paratelum and telum very short: sheaths of the oviduct just appearing beyond the tip of the abdomen. Length of the body 2 lines; expansion of the wings 4 lines.

Page 48. Pтеromalus Sunides. Viridis cupreo varius, antennis piceis, pedibus flavis, alis limpidis.

Male; Green, varied with cupreous, shining; head and thorax finely squameous : head broader than the thorax : antennæ slender, filiform, piceous, longer than the thorax; first joint yellow; the joints from the fifth to the tenth successively decreasing in length; club fusiform, more than twice the length of the tenth joint: thorax fusiform: prothorax of moderate size : sutures of the parap. sides indistinct; scutellum exhibiting no traces of the transverse suture: propodeon large, with a slight keel along the middle: podeon extremely short: abdomen fusiform, smooth, shorter than the thorax; metapodeon large; uctoon, ennaton and decaton of moderate size; protelum and following segments very short: legs yellow: wings limpid; nervures fulvous; humerus much longer than the ulna; radius shorter than the ulna; cubitus shorter than the radius; stigma very small.

Female; Dull-green : head as broad as the thorax: antennæ subclavate, rather shorter than the thorax ; first joint piceous, fulvous at the base; club oval: abdomen oval, a little shorter and broader than the thorax, concave above, keeled beneath. Length of the body $1 \frac{1}{2}$ line; expansion of the wings $2 \frac{1}{2}$ lines.

Page 48. Pteromalus Odites. Purpureus, abdomine cyaneo basi viridi, antennis piceis, pedibus flavis, femoribus piceo vittatis, alis limpidis.

Female; Dull purple, head and thorax finely squameous; head a little broader than the thorax : antennæ subclavate, slender, piceous, much shorter than the thorax; first joint fulvous : the joints from the fifth to the tenth successively decreasing in length; club conical, much longer than the tenth joint: thorax fusiform: prothorax of moderate size: sutures of the parapsides very indistinct; no traces of the suture across the scutellum : propodeon green, keeled lengthwise: podeon extremely short: abdomen oval, smooth, shining, coucave above, keeled beneath ; shorter and broader than the thorax; disk chiefly blue; metapodeon large, bright-green, varied with cupreous; octoon and following segments of moderate size: legs yellow; a pale piceous stripe along each thigh : wings limpid; nervures yellow; humerus much longer than the ulna; radius shorter than the ulna; cubitus shorter than the radius; stigma fulvous, very small. Length of the body $1 \frac{1}{2}$ line; expansion of the wings 2 lines. 
Page 48. Pteromalus Nestocles. Viridis, abdomine cupreo, antennis piceis, pedibus fulvis, femoribus viridibus, alis limpidis.

Male; Body stout, dull green: head 'and thorax finely squameous: head broader than the thorax: antennæ filiform, slender, piceous, hardly longer than the thorax; joints from the fifth to the tenth successively decreasing in length; club fusiform, twice the length of the tenth joint: thorax oval: prothorax short: sutures of the parapsides indistinct, scutellum with no traces of a transverse suture: propodeon large, not keeled: podeon extremely short : abdomen nearly linear, depressed, smooth, shining, shorter than the thorax; disk cupreous; metapodeon large; octoon and following segments of moderate size: legs fulvous; coxæ and thighs green, tips of the latter fulvous: wings limpid; nervures fulvous; humerus much longer than the ulna; radius shorter than the ulna; cubitus shorter than the radius; stigma very small. Length of the body $1 \frac{1}{4}$ line; expansion of the wings $1 \frac{3}{4}$ line.

Page 48. Pteromalus Amyntor. Cyaneoviridis, thorace aneo, antennis fuscis, pedibus fulvis, femoribus viridibus, alis subflavis.

Female; Bright-bluish-green: head and thorax finely squameous: head as broad as the thorax : antennæ clavate, fuscous, shorter than the thorax; first joint fulvous; joints from the fifth to the tenth successively decreasing in length; club conical, twice the length of the tenth joint: thorax oval, bright æneous : prothorax very short; sutures of the parapsides very indistinct; no traces of the transverse suture on the scutellum: propodeon short, keeled: podeon extremely short : abdomen fusiform, concave above, keeled beneath, longer than the thorax; hind-border of each segment cupreous; metapodeon large; octoon and following segments of moderate size: legs bright fulvous; coxæ and thighs green, tips of the latter fulvous; tips of the tarsi piceous : wings slightly tinged with yellow; nervures yellow; humerus rather more than twice the length of the ulna; radius a little longer than the ulna; cubitus shorter; stigma fuscous, very small. Length of the body $\frac{3}{4}-1$ line; expansion $1 \frac{1}{4}-1 \frac{1}{2}$ line.

Page 48. Pteromalus Cercides, Mas. et Fem. Viridis, abdomine cupreo, antennis nigris, pedibus fulvis, femoribus viri-

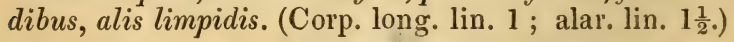

Body nearly linear: head and thorax dark-green, finely squameous: head broader than the thorax: antennæ black, subclavate, slender, as long as the thorax, first joint long, very slender; second cyathiform; third and fourth very minute; the following 
joints from the fifth to the tenth successively decreasing in length; club fusiform, more than twice the length of the tenth joint : thorax oval: prothorax transverse, very short: scutum of the mesothorax broad; sutures of the parapsides indistinct ; scutellum obconic, having a slight trace of a transverse suture near the tip: propodeon obconic, declining, bluish-green: podeon very short: abdomen dark cupreous, depressed above, increasing in breadth from the base to the tip, shorter and narrower than the thorax; metapodeon of moderate length, varied with green; octoon and following segments short: legs fulvous; coxæ and thighs green; tips of the latter fulvous: wings limpid; nervures fuscous; ulna rather more than half the length of the humerus; radius much shorter than the ulna; cubitus nearly as long as the radius; stigma very small.

Female; Abdomen oval, keeled beneath.

Page. 52. Epistenia Feretrius, Walker, MSS. Nigra, antennis basi rufis, pedibus rufis, coxis femoribusque nigro cyaneis, tarsis fulvis, alis limpidis.

Body convex, black, with a very slight metallic tinge, dull, punctured, pubescent : head transverse, short, a little broader than the thorax; vertex broad; front abruptly declining, excavated: eyes of moderate size : ocelli 3 , disposed in a triangle on the vertex : antennæ black, subclavate, mutilated ; first joint long, slender, red towards the base : thorax oval : prothorax transverse, very short : scutum of the mesothorax large; sutures of the parapsides indistinct ; scutellum large, obconical : metathorax and propodeon very short, almost hidden by the scutellum: podeon extremely short: abdomen subulate, finely punctured, slightly shining, narrower than the thorax, and nearly twice its length; hind-borders of the segments almost smooth; octoon, ennaton and decaton short; protelum much longer ; paratelum and telum dart-shaped ; oviduct projecting a little beyond the tip of the latter: legs slender, pubescent; coxæ and thighs bluish-black; trochanters, knees, and tibiæ dull-red; tarsi fulvous : wings limpid; nervures piceous; humerus nearly twice the length of the ulna; radius rather shorter than the ulna; cubitus not half the length of the radius; stigma very small. Length of the body $4 \frac{1}{2}$ lines; expansion of the wings 5 lines.

Epistenid Ania, Walker, MSS. Purpurea, antennis nigris basi rufis, coxis, metafemorumque vittis purpureis, alis subfuscis.

Female; Body convex, dull, punctured, purple, pubescent, head transverse, short, blue, as broad as the thorax; vertex broad; front green, excavated, abruptly declining: antennæ black, fili- 
form, shorter than the thorax ; first joint bluish-green, long, slender, red towards the hase: thorax elliptical: prothorax transverse, very short: scutum of the mesothorax large ; sutures of the parapsides indistinct; scutellum large, obconical: metathorax and propodeon transverse, short : abdomen cylindrical, very long and slender, about twice the length of the thorax : legs red; coxæ purple; a purple stripe along each hind-thigh: wings with a slight fuscous tinge; nervures piceous; humerus much longer than the ulna; radius shorter than the ulna; cubitus short; stigma very small. Length of the body 9 lines; expansion of the wings 6 lines.

Page 52. Calosoter Anemetus, Walker, MSS. Purpureus cyaneo et viridi varius, antennis nigris, pedibus rufis, alis limpidis.

Body convex, long, very slender, purple, tinged with blue and green; head and thorax punctured: head transverse, short, not broader than the thorax; vertex rather narrow; front abruptly declining, excavated, eyes of moderate size, prominent : ocelli forming a small triangle on the vertex: antennæ black, mutilated: thorax elliptical : prothorax very narrow, almost smooth, attenuated in front, much lower than the mesothorax: scutum of the mesothorax large; sutures of the parapsides very indistinct ; scutellum obconical, of moderate size : metathorax and propodeon very short, transverse, declining: abdomen dart-shaped, shining, very finely squameous, keeled beneath, much longer and narrower than the thorax, slightly compressed; segments rather long oviduct red, not passiug the tip of the abdomen : legs red, slender, slightly pubescent: wings limpid; nervures piceous; humerus rather more than twice the length of the ulna; radius shorter than the ulna; cubitus curved towards the radius, hut less than half its length ; stigma extremely small. Length of the body 5 lines; expansion of the wings 6 lines.

Eupelmus Iopas, Walker, MSS. Fem. Purpureus, capite viridi, abdomine cyaneo et aneo vario, antennis nigris basi fulvis, oviductu abdomine breviore, pedibus fulvis, alis limpidis. (Corp. long. lin. 2 ; alar. lin. $2 \frac{1}{2}$.)

Head dark-green, transverse, pubescent, bluish in front, as broad as the thorax : antennæ slender, subclavate, black, a little longer than the thorax; first joint long, slender, dull fulvous; second long-cyathiform; third and fourth very minute; fifth and following joints to the tenth long, successively shorter and broader; club fusiform, compressed diagonally at the tip, twice tine length of the tenth joint: thorax elliptical, purple, brighter beneath, in structure like that of $E$. urocerus : podeon very short: abdomen fusiform, purple varied with blue and æneous, longer and a little narrower than the thorax, in structure like that of $E$. urocerus : 
oviduct exerted ; its sheaths fulvous, a little shorter than the abdomen, black along one-third of their length from the hase; legs fulvous; tarsi pale yellow; middle-legs long and dilated as usual ; wings rather small, limpid from the base till near the middle, thence fuscous till near the tips; nervures piceous; humerus much less than half the length of the wing; ulna as long as the humerus; radius not more than one-fourth of the length of the ulna; cubitus as long as the radius; stigma very small, emitting a short branch.

Encretus Polylaus, Fem. Viridis, abdomine cupreo, antennis fuscis, metapedibus fuscis aut piceis, alis limpidis. (Corp. long. lin. $\frac{3}{4}$; alar. lin. $1 \frac{1}{2}$.)

Body rather long; head and thorax convex, bright-green, finely squameous; head transverse, as broad as the thorax; vertex rather broad ; front not impressed ; eyes of moderate size, not prominent ; ocelli disposed in a triangle on the vertex ; antennæ slender, fuscous,subclavate, rather longer than the thorax; first joint long, slender, yellow ; second piceous, cyathiform; third and following joints to the club successively increasing in breadth; club fusiform; thorax elliptical; prothorax transverse, short, rounded in front, straight behind ; scutum of the mesothorax large, transverse; parapsides united to the scutum; axillæ large, complete; scutellum large, obconic ; metathorax, propodeon, and podeon very short, declining; abdomen cupreous, obconic, depressed, smooth, shining, narrower and much shorter than the thorax; metapodeon bright-green, of moderate size; octoon and following segments short; legs fulvous, stout; middle-thighs and middle-tibiæ dilated, and the former armed with long spines; hind thighs and hind tibiæ slightly fuscous; wings limpid ; nervures fulvous; humerus much less than half the length of the wing; ulna and radius extremely short; cubitus longer than the radius; stigma very small.

a. England. Presented by F. Walker, Esq.

Encyrtus, Nasidius, Walker, MSS. Fem. Ater, abdomine aneo, antennis fuscis aut piceis, pedibus fulvis aut piceis, alis limpidis. (Corp. long. lin $\frac{1}{3}$; alar. lin. $\frac{3}{4}$.)

Borly stout, pubescent: head and thorax slightly convex, deephlack, dull, squameous; head transverse, very short, not narrower than the thorax; vertex broad; front impressed, abruptly declining; eyes of moderate size, not prominent; ocelli near together in a triangle on the vertex ; antennæ clavate, fuscous, as long as the thorax ; first joint piceous, long, slender ; second piceous, long-cyathiform ; third and following joints to the eighth successively increasing in breadth; club fusiform, broader than the eighth joint 
and more than twice its length; thorax short, nearly oval; prothorax very short; scutum of the mesothorax broad; parapsides united to the scutum; axillæ complete ; scutellum large, obconic ; propodeon short; podeon extremely short; abdomen obconic, depressed, æneous, smooth, shining, a little shorter and broader than the thorax; metapodeon and following segments short ; legs fulvous; protibiæ and protarsi dilated, and the former armed with long spines; coxæ black; thighs and tibiæ piceous, tips of the latter fulvous; wings limpid, rather large ; nervures fulvous; humerus much less than half the length of the wing; ulna and radius broad, very short; cubitus of moderate length; stigma very small. Var. B. Antennæ piceous; abdomen æneous-black; tibiæ altogether piceous; tarsi fuscoús.

a. England. Presented by F. Walker, Esq.

Encyrtus Proculus, Walker, MSS. Fem. Viridis, abdomine antennis pedibusque nigris, genubus tarsisque flavis, alis limpidis. (Corp. long. lin. $\frac{1}{2}-\frac{2}{3}$; alar. lin. $\frac{3}{4}-1$.)

Body thick; head and thorax convex, squameous, bright-green ; head a little broader than the thorax; vertex broad; front not impressed, abruptly declining ; eyes of moderate size, not prominent; ocelli disposed in a triangle on the vertex : antennæ clavate, black, inserted near the mouth, nearly as long as the body ; first joint slender, very long; second long-cyathiform; the following joints from the third to the eighth successively shorter and broader; club fusiform, broader than the eighth joint and thrice its length; thorax short, oval; prothorax very short; scutum of the mesuthorax very large, narrower in front; parapsides united to the scutum ; axillæ complete; scutellum somewhat obconic; propodeon short, declining; podeon extremely short; abdomen oval, depressed, shining, finely squameous, æneous black, narrower than the thorax and hardly more than half its length; metapodeon, octoon, ennaton and decaton of moderate size; the other segments very short; oviduct exerted, as long as one-fourth of the abdomen ; legs black, stout; mesotibiæ and mesotarsi dilated, and the former armed with long spines; coxæ green; knees yellow ; tarsi yellow, their tips piceous; wings limpid; nervures piceous; humerus much less than half the length of the wing; ulna and radius broad, extremely short; cubitus of moderate length, pointing towards the disk of the wing; stigma very small.

a. England. Presented by F. Walker, Esq.

Encyrtus Fundulus, Walker, MSS. Mas. Cupreus scuto viridi, abdomine nigro, antennis fuscis basi piceis, pedibus fusco-flavis, nigro cinctis, alis limpidis. (Corp. long. lin. $\frac{1}{2}$; alar. lin. 1.)

Body linear, rather narrow; head and thorax convex, finely 
squameous: head transverse, æneous, broader than the thorax vertex broad; front abruptly declining, not impressed: eyes of moderate size, not prominent : ocelli forming a triangle on the vertex : antennæ subclavate, slender, fuscous, longer than the thorax : first joint long, stout, piceous; second piceous, longcyathiform; the following joints from the third to the eighth successively increasing in length ; club fusiform, broader than the eighth joint, and more than twice its length: thorax oblongsubquadrate, dark cupreous: prothorax transverse, very short: scutum of the mesothorax broad, bright green: parapsides united to the scutum; axillæ complete; scutellum obconic : propodeon subquadrate, declining, rather large: podeon extremely short: abdomen linear, depressed, black, minutely squameous, rather shorter and narrower than the thorax; metapodeon of moderate length; octoon and following segments short: legs black; mesotibiæ and mesotarsi slightly dilated, and the former armed with long spines; tips of the tibiæ yellow; trochanters, protibiæ, protarsi, and metatarsi fuscous; knees and mesotarsi yellow, tips of the latter fuscous : wings limpid, rather narrow; nervures fuscous; humerus much less than half the length of the wing; ulna and radius broad, very short; cubitus of moderate length, pointing towards the disk of the wing; stigma very small.

a. Scotland. Presented by F. Walker, Esq.

Page 61. Elasmus Anius. Fem. Niger, capite viridi, antennis piceis, pedibus fuscis, femoribus nigris, protibiis fulvis, alis

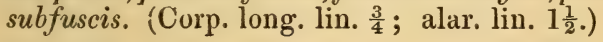

Body black: head and thorax convex, duil, roughly punctured: head green, very short, nearly as broad as the thorax : eyes of moderate size, not prominent: antennæ piceous, subclavate, as long as the thorax ; first joint long, slender ; second long-cyathiform; third and fourth very minute; fifth, sixth, and seventh linear, nearly equal in size; club fusiform, twice the length of the seventh joini: thorax oval: puthorax transverse, very short: scutum of the mesothorax broad; sutures of the parapsides indistinct; scutellum obconic: metathorax transverse, very short: podeon very short: abdomen lanceolate, smooth, shining, compressed, concave above, keeled beneath, narrower and much longer than the thorax; metapodeon of moderate length; octoon and following segments short: legs fuscous, long, slender; coxæ and thighs black; mesotibiæ fulvous: wings slightly fuscous, narrow; nervures fuscous; ulna nearly twice the length of the humerus; radius short; cubitus not more than half the length of the radius; stigma very small.

a. England. Presented by F. Walker, Esq. 
Page 69. Elachestus Idomene, Walker, MSS. Eulophus Artæus, Walker, Mon. Chal. ii. 41. Aneoviridis, abdomine basi flavo maculato, antennis nigris, pedibus fulvis, alis limpidis.

Female; Body depressed : head and thorax finely squameous, æneous green : antennæ black, filiform, more than half the length of the body; first joint fulvous, long, slender : prothorax welldeveloped, narrower in front: sutures of the parapsides indistinct; axillæ large : metathorax furrowed: abdomen elliptical, æneous, smooth, much shorter than the thorax, with a large pale yellow spot near its base: legs pale fulvous: wings limpid; nervures piceous; humerus much longer than the ulna; radius much shorter than the ulna; cubitus shorter than the radius; stigma very small. 


\section{LIST OF GENERA.}

Agamerion, $\%$.

Agaon, 23.

Agonioneurus, 60 .

Amblymerus, 37.

Aneure, 61.

Aphelinus, 60.

Aprostocetus, 78 .

Asaphes, 23.

Bellerus, 72.

Brachymeria, 5 .

Callimome, 15.

Calliptiles, 61.

Calosoter, 52, 92.

Calypso, 24.

Caratomus, 23.

Cerchysius, 54 .

Cercobelus, 54 .

Cerocephala, 23.

Chalcis, 4, 83.

Cheiloneurus, 54 .

Cheiropachus, 50 .

Choreus, 59.

Chrysolampus, 13, 29.

Cinipsyllum, 22.

Cirrospilus, 72.

Cleonymus, 52.

Cleptes, 42.

Closterocerus, 62.

Coccophagus, 60.

Coryna, 29.

Corynocere, 24.
Cratomus, 23.

Cyrtogaster, 26.

Cyrtosoma, 20.

Decatoma, 12.

Derostenus, 62.

Dicladocerus, 72.

Dicyclus, 27.

Diomorus, 15.

Dirhinus, 7, 85 .

Elachestus, 68, 96 .

Elasmus, 61, 96.

Encyrtus, 54, 93.

Entedon, 61.

Epistenia, 52, 91.

Epitranus, 4.

Ericydnus, 53.

Eucharis, 21, 85.

Euderus, 67.

Eulophus, 69.

Eupelmus, 52, 93.

Euplectrus, 67 .

Eurytoma, 8, 85.

Eutelus, 36.

Gastrancistrus, 24.

Glyphe, 25.

Haltichella, 6, 84.

Hemiptarsenus, 71 .

Hetroxys, 50.

Hockeria, 6. 
Horismenus, 66.

Idarnes, 23.

Isocyrtus, 34.

Isosoma, 11 .

Lamprotatus, 29, 89.

Lelaps, 52.

Leucospis, 1.

Loesthia, 23.

Lophocomus, 67.

Macroglenes, 24.

Macroneura, 51.

Marres, 2.

Megastigmus, 14.

Megorismus, 29.

Meraporus, 35.

Merismus, 28.

Merostenus, 51.

Mesopolobus, 36.

Metapelma, 53.

Micradelus, 26.

Micromelus, 35.

Miscogaster, 7, 25.

Monodontomerus, 14.

Myina, 60.

Norbanus, 35.

Notaspis, 7 .

Omphale, 65.

Ophelimus, 67.

Ormocerus, 26.

Ormyrus, 20.

Pachylarthrus, 26.

Pachyneuron, 35.

Palmon, 14.

Panstenon, 29.
Paphagus, 29.

Periglyphus, 20.

Perilampus, 22, 88.

Phacostomus, 26.

Phagonia, 26.

Phasgonophora, 6 .

Pirene, 24.

Platyterma, 36.

Priomerus, 14.

Prosodes, 28.

Prosopon, 51.

Psilocera, 36.

Pteromalus, 36, 89.

Pteroptrix, 61.

Schizaspidia, 22.

Seladerma, 33.

Semiotus, 34 .

Siphonura, 20.

Smaragdites, 65.

Smiera, 2.

Spalangia, 23.

Sphegigaster, 28.

Sphenolepis, 59.

Stenomesius, 68.

Stenophrus, 24.

Syntomopus, 28.

Systasis, 34 .

Systole, 11.

Tetrastichus, 73.

Theocolax, 23.

Thoracantha, 22, 87.

Torymus, 14.

Toxeuma, 28.

'Trichogramma, 61.

Trigonoderus, 51.

Urolepis, 26. 





\section{I S T}

OF THE

\section{S P E C I M E N}

OF

\section{HYMENOPTEROUS INSECTS}

IN

THE COLLECTION

OF THE

\section{BRITISH MUSEUM.}

PART II. - CHALCIDITES.

ADDITIONAL SPECIES.

PRINTED BY ORDER OF THE TRUSTEES.

LONDON, 1848. 


\section{LONDON :}

PRINTED BY EDWARD NEWMAN, 9, DFVONSHIRE ST. BISHOPSGATE. 


\section{INTRODÚCTION.}

THE principal object of the present Catalogue has been to give a complete list of the specimens of Hymenopterous Insects contained in the collection of the British Museum, indicating at the same time the peculiarities of each, as regards variations in colour, locality, and the source from whence it has been derived.

With this view, the different individuals of each species contained in the collection are indicated by the letters $a, b, c$, \&c., following the name of the species, and its synonymes.

In the adoption of generic and specific names, it has been thought right to use, wherever it was possible, that which was first applied to the species. As far as regards the specific names, there is comparatively little difficulty in the application of this simple rule; but generic names have been used by different authors in senses so widely different, and the groups which they were meant to designate have been so variously extended or restricted, that it is no easy matter to determine, where several names have been used, which of them ought to be preferred. 
If the generic name adopted be different from that first employed for the species after the establishment of the Linnean system of nomenclature, the name under which it was first described follows as a synonyme, and where the Insect has received more than one specific name, those names are also given, as heing sufficient for all purposes of identification.

These are followed by a reference to a few of the best original figures of the species.

In those cases where the two sexes of the same species, or any particular individual state or variety belonging to it, have been differently named, the name or figure exclusively appertaining to the state or individual is placed after the indication of the specimen to which it applies.

This part, containing the Chalciditous Insects of this order which have been procured chiefly from Mr. F. Walker since the former part was published, has been kindly prepared by that gentleman, who has devoted his attention to these Insects, and has been the original describer of the greater number of the species. The Appendix of this part, also written by Mr. Walker, contains the descriptions of the hitherto undescribed species contained in the collection.

The part containing the other families will follow as soon as they are prepared.

JOHN EDWARD GRAY.

British Museum, April 20th, 1848. 


\section{LIST OF CHALCIDITES.}

\section{SUPPLEMENT。}

\section{Order-CHALCIDITES.}

Family. Leucospide.

Levcospis, Fabr.

Levcospis Cayennensis? Westwood, Germar. Zeit. Ent. t. 4, f. 4.

a. Isle St. Thomas. From Mr. Walker's collection.

\section{Fam. Chalcide.}

Smiera, Spinola.

Smiera nigrifex, Walker, Cat. Chalc. B. M. p. 2.

l. Prussia. Presented by E. Doubleday, Esq.

$m$. Prussia. From Mr. Walker's collection.

$$
\text { Chalcis, Fabr. }
$$

Chalcis intermedia, Cat. Chalc. B. M.p.4. Nees. Hym. Ich. aff. Mon. ii. 29, 8.

a. Prussia. Presented by E. Doubleday, Esq.

b. Prussia. From Mr. Walker's collection.

\section{Fam. Eurytomide.}

$$
\text { Eurytoma, } I l l \text {. }
$$

Eurytona atra. Isosoma atrum, Walker, Ent. Mag. i. 14.

a. England. From Mr. Walker's collection. 
Eurytoma Cestius, Walker, MSS. See Appendix.

a. England. From Mr. Walker's collection.

Eurytoma Mucianus, Walker, MSS. See Appendix. a. England. From Mr. Walker's collection.

\section{Isosoma, Walker.}

Isosoma tenuicorne, Walker, Ent. Mag. i. 19. a. England. From Mr. Walker's collection.

Isosoma pusillum, Walker, Ent. Mag. i. 20. a. England. From Mr. Walker's collection.

Isosoma breviventre, Walker, Ent. Mag. i. 20.

a. England. From Mr. Walker's collection.

Isosoma vacillans, Walker, Ent. Mag. iv. 23.

a. England. From Mr. Walker's collection.

Isosoma Nepe, Walker, Ann. Nat. Hist. xiv. 21.

a. England. From Mr. Walker's collection.

\section{Decatoma, Spin.}

Decatoma plana, Walker, Ent. Mag. i. 27. a. England. From Mr. Walker's collection.

Decatoma tenuicornis, Walker, Ent. Mag. i. 28.

a. England. From Mr. Walker's collection.

Decatoma mesomelas, Walker, Ent. Mag. iv. 24.

a. England, From Mr. Walker's collection.

Decatoma flavicornis, Walker, Ent. Mag. iv. 25.

a. England. From Mr. Walker's collection.

Decatoma Nicææ, Walker, Ann. Nat. Hist. xiv. 22.

a. England. From Mr. Walker's collection.

Fam. Torymid

Megastiguus, Spin.

Megastigmus dorsalis, Cat. Chalc. B.M. p. 14 Torymus stigma, Nees. Hym. Ich. aff. Mon. ii. 65, 15. T. giganteus, var.? Kollar.

c. Prussia. Presented by E. Doubleday, Esq.

d. Prussia. From Mr. Walker's collection. 


\section{Monodontomerus, Westw.}

Monodontomerus stigma. Torymus ater, Nees. Hym. Ich. aff. Mon. ii. 69, 21.

a. Prussia. Presented by E. Doubleday, Esq.

$b$. Prussia. From Mr. Walker's collection.

Monodontomerus cupreus. Torymus cupreus, Nees. Hym. Ich. aff. Mon. ii. 67, 18. Monodontomerus æreus, De Laporte, Walker, Ent. Mag. ii. 158.

a. Prussia. Presented by E. Doubleday, Esq.

$b$. Prussia. From Mr. Walker's collection.

Monodontome rus obsoletus. Torymus obsoletus, Nees. Hym. Ich. aff. Mon. ii. 68, 19. ? Dipl. obsoleta, Fabr. Syst. Piezat. 150, 10. Spin. Ins. Lig. Fasc. iii. 159, 8. Fasc. iv. 213. Ann. Mus. vii. 148. Cynips obsoleta, Latr. Gen. Cr. et Ins. iv. 28. Ich. obsoletus, Fabr. Ent. Syst. Suppl.218, 230 ? Coqueb. Ill. con. i. 21. Tab. 5, f. 2. Ich. rodophthalmus, Rossi, Mant. i. 125, 276.

a. Prussia. From Mr. Walker's collection.

\section{Callimome, Spin.}

Callimome cynipedis, Cat. Chalc. B. M. p. 15.

c. Prussia. From Mr. Walker's collection.

Callimome cingulatus, Cat. Chalc. B. M. p. 15.

c. Prussia. Presented hy E. Doubleday, Esq.

d. Prussia. From Mr. Walker's collection.

Callinome regalis, Walker, Ent. Mag. i. 119.

a. England. From Mr. Walker's collection.

Callimome Bedeguaris, Cat. Chalc. B. M. p. 16.

b. Prussia. Presented by E. Doubleday, Esq.

c. Prussia. From Mr. Walker's collection.

Callimome elegans, Cat. Chalc. B.M.p. 16. Torymus auronitens, Foerster, Mon. Pterom. i. xxx. 14.

d. Prussia. From Mr. Walker's collection.

Callimome nigricornis, Cat. Chalc. B. M. p. 17.

c. Prussia. Presented by E. Doubleday, Esq.

d. Prussia. From Mr. Walker's collection.

Callinome mutabilis, Cat. Chalc. B.M. $p .17$. Torymus compressus, Foerster, Mon. Pterom. i. xxxii. 6.

b. Prussia. Presented by E. Doubleday, Esq.

c. Prussia. From Mr. Walker's collection. 
Callimome regius, Cat. Chalc. B. M. p. 19. C. inconstans, De Lap., \&c. Torymus regius, Nees. Hym. Ich. aff. Mon. ii. 55, 1. De Geer, Ins. ii. T. incertus, Foerster, Mon. Pterom. 1. xxxi. 25, 187. t. 31, f. 21. T. pubescens, Foerster, Mon. Pterom. 1. xxx. 15.

a. Prussia. Presented by E. Doubleday, Esq.

b. Prussia. From Mr. Walker's collection.

Callimome Rasaces, Walker, Ann. Nat. Hist. xiv. 181.

a. England. From Mr. Walker's collection.

Callimome formosus, Walker, Ent. Mag. i. 122.

a. England. From Mr. Walker's collection.

CaLlimome macropterus, Walker, Ent. Mag. i. 124.

a. England. From Mr. Walker's collection.

Callimome nitens, Walker, Ent. Mag. i. 126.

a. England. From Mr. Walker's collection.

Callimome brevicauda, Walker, Ent. Mag. i. 126.

a. England. From Mr. Walker's collection.

Callimome microcerus, Walker, Ent. Mag. i. 128.

a. France. From Mr. Walker's collection.

Callimome micropterus, Walker, Ent. Mag. i. 130.

a. England. From Mr. Walker's collection.

Callimome viridiæneus, Walker, Ent. Mag. i. 131

a. England. From Mr. Walker's collection.

Callimome moestus, Walker, Ent. Mag. i. 133.

a. England. From Mr. Walker's collection.

Callimome stramineitarsus, Walker, Ent. Mag. i. 135.

a. England. From Mr. Walker's collection.

Callimome ater, Walker, Ent. Mag. i. 138.

a. Eugland. From Mr. Walker's collection.

Callimome fuscipennis, Walker, Ent. Mag. i. 137.

a. England. From Mr. Walker's collection.

Callimome nigritarsus, Walker, Ent. Mag. i. 134.

a. England. From Mr. Walker's collection.

Callimome Angelicæ, Walker, Ent. Mag. iv. 25.

a. England. From Mr. Walker's collection.

Callimome Erope, Walker, Ann. Nat. Hist. xiv. 182.

a. England. From Mr. Walker's collection.

Callimome meridionalis, Walker, Ent. Mag. i. 131.

a. France. From Mr. Walker's collection. 
Callimome arvernicus, Walker, Ent. Mag.i. 134.

a. France. From Mr. Walker's collection.

Callimome Euphorbiæ, De Laporte, Ent. Mag. i. 131.

a. France. From Mr. Walker's collection.

Callimome eurynotus. Torymus eurynotus, Foerster, MSS. Walker, Ann. Nat. Hist.

a. Prussia. Presented by E. Doubleday, Esq.

b. Prussia. From Mr. Walker's collection.

Callimome chrysis. Torymus chrysis, Nees. Hym. Ich. aff. Mon. ii. 67,17 .

a. Prussia. Presented by E. Doubleday, Esq.

$b$. Prussia. From Mr. Walker's collection.

Callimome Nepthys, Walker, Ann. Nat. Hist. 1848.

a. England. From Mr. Walker's collection.

Callimome Solinus, Walker. See Appendix.

a. England. From Mr. Walker's collection.

\section{Ormyrus, Westw.}

Ormyrus punctiger, Cat. Chalc. B. M. p. 20. Siphoneura variolosa, Nees. Hym. Ich. aff. Mon. ii. 82, 1.

d. Prussia. Presented by E. Doubleday, Esq.

e. Prussia. From Mr. Walker's collection.

Ormyrus cæruleus. Siphonura cærulea, Foerster, MSS. Walker, Ann. Nat. Hist. 1848.

a. Prussia. From Mr. Walker's collection.

Ormyrus nigrocyaneus, Walker. Ent. Mag. i. 140.

a. England. From Mr. Walker's collection.

Fam. Eucharid

Eucharis, Latr.

Edcharis adscendens, Cat. Chalc. B. M. p. 21.

c. Germany. From Mr. Walker's collection.

Perilampus, Latr.

Perilampus micans, Dalm, Kongl. Vet. Acad. Handl. 1822.

a. Germany. From Mr. Walker's collection. 
Fam.

Elatus, Walker, MSS. See Appendix.

Elatus Thenæ, Walker, MSS. See Appendix.

a. England. From Mr. Walker's collection.

Fam. Spalanginde.

Spalangia, Latr.

Spalangia nigra, Cat. Chalc. B. M. p. 23. S. nigripes, S. nigroænea, Curtis, Brit. Ent. 140.

e. England. From Mr. Walker's collection.

Spalangia hirta, Haliday, Ent. Mag. i. 334.

a. England. From Mr. Walker's collection.

Cerocephala, Wesiw.

Cerocephala cornigera. C. cornigera, Walker, Ent. Mag. ii. 149 .

a. Germany. From Mr. Walker's collection.

Fam. Pteromalide.

Asaphes, Walker,

Asaphes ænea. Chrysolampus suspensus, Nees. Hym. Ich. aff. Mon. ii. 127. C. altiventris, Nees. Hym. \&c. ii. 127. Podagrion splendens? Spin. Ann. Mus. vii. 147, 2. Pteromalus petiolatus? Zett. Ins. Lapp. xiv. 332. Colax Aphidii, Curtis, Journ. Roy. Agric. Soc. iii.

a. England. From Mr. Walker's collection.

Tetramesa, Walker, MSS. See Appendix.

Tetramesa Larbas, Walker, MSS. See Appendix.

a. England. From Mr. Walker's collection.

Gastrancistrus, Westw.

Gastrancistrus viridis, Walker, Ent. Mag. ii. 173.

a. England. From Mr. Walker's collection.

Gastrancistrus tenebricosus, Walker, Ent. Mag. ii. 174.

a. England. From Mr. Walker's collection.

Gastrancistrus obscurellus, Walker, Ent. Mag. ii. 17

a. England. From Mr. Walker's collection. 
Gastrancistrus Acontes, Walker, Ann. Nat. Hist. iv. 32.

a. Scotland. From Mr. Walker's collection.

Gastrancistrus longicornis, Haliday, MSS. See Appendix.

a. Ireland. Erom Mr. Walker's collection.

Gastrancistrus Dryas, Walker, Mon. Chal. i. 209.

a. South of France. From Mr. Walker's collection.

Gastrancistrus Panares, Walker, Ann. Nat. Hist. xiv. 339.

a. Alten, Finmark. From Mr. Walker's collection.

Gastrancistrus Amabœus, Walker, MSS. See Appendix.

a. England. From Mr. Walker's collection.

Gastrancistrus Accia. Walker, MSS. See Appendix.

a. England. From Mr. Walker's collection.

Gastrancistrus Hamillus, Walker, MSS. See Appendix.

a. England. From Mr. Walker's collection.

Gastrancistrus Geganius, Walker, MSS. See Appendix.

a. England. From Mr. Walker's collection.

Gastrancistrus Fillenas, Walker. See Appendix.

a. England. From Mr. Walker's collection.

Gastrancistrus Alectus, Walker. See Appendix.

a. England. From Mr. Walker's collection.

Gastrancistrus Hierocles, Walker. See Appendix.

a. England. From Mr. Walker's collection.

Gastrancistrus Iriarte, Walker. See Appendix.

a. England. From Mr. Walker's collection.

Gastrancistrus Amnisos, Walker. See Appendix.

a. England. From Mr. Walker's collection.

Gastrancistrus Pacilus, Walker, MSS. See Appendix.

a. England. From Mr. Walker's collection.

Gastrancistrus Lœlianus, Cat. Chalc. B. M. See Appendix.

a. England. From Mr. Walker's collection.

\section{Psilonotus, Walker.}

Psilonotus Adamas, Walker, Ent. Mag.ii. 179. The Entomologist, pl. B. fig. 2.

a. England. From Mr. Walker's collection.

Psilonotus Hortensia, Haliday, Ann. Nat. Hist. 1846.

a. Ireland. From Mr. Walker's colleçion.

Psilonot us Achæus, Walker, MSS. See Appendix.

a. England. From Mr. Walker's collection. 
ACrias, Walker.

Acrias Nileus, Walker, Ann. Nat. Hist. 1848, 29.

a. St. John's Bluff, Florida. Presented by E. Doubleday, Esq.

Rhaphitelus, Walker.

Rhaphitelus maculatus, Walker, Ent. Mag. ii. 179. The Entomologist, pl. A, fig. 2. Pteromalus subulifer, Foerster, Mon. Pterom. i. xxviii. 264. Stylocerus subulifer, Erichson, Bericht. 1847.

a. England. From Mr. Walker's collection.

Macromesus, Walker, MSS. See Appendix.

Macromesus Amphiretus, Walker, MSS. See Appendix. a. England. From Mr. Walker's collection.

\section{Meromalus, Walker.}

Meromalus flavicornis, Walker, Ent. Mag. ii. 178. Ormocerus Drymo, Mon. Chal. i. 205.

a. England. From Mr. Walker's collection.

Macroglenes, Westw.

Macroglenes penetrans, Cat. Chal. B. M. p. 24. Trans. Ent. Soc. iii. 295. Curtis, Journ. Roy. Agric. Soc. v.

c. Prussia. Presented by E. Doubleday, Esq.

d. Prussia. From Mr. Walker's collection.

\section{Pirene, Haliday.}

Pirene Scylax, Walker, MSS. See Appendix.

a. England. From Mr. Walker's collection.

Pirene varicornis. Hal. Trans. Ent. Soc. iii. 296.

a. England. From Mr. Walker's collection.

Pirene chalybea, Hal. Trans. Ent. Soc. iii. 296.

a. England. From Mr. Walker's collection.

Pirene eximia, Hal. Trans. Ent. Soc. iii. 296.

a. England. From Mr. Walker's collection.

Ormocerus, Walker.

Ormocerus Sabbas, Walker, MSS. See Appendix.

a. England. From Mr. Walker's collection. 
Ormocerus Aletes, Walker, MSS. See Appendix.

a. England. From Mr. Walker's collection.

Ormocerus Borges, Walker, Mon. Chal. i. 208.

a. Ireland. From Mr. Walker's collection.

Ormocerus Bambyce, Walker, Mon. Chal. i. 208.

a. England. From Mr. Walker's collection.

Ormocerus Vibulenus, Walker, Mon. Chal. i. 205.

a. England. From Mr. Walker's collection.

Ormocerus Mandrocles, Walker, Mon. Chal. i. 206.

a. England. From Mr. Walker's collection.

Ormocerus Pisuthrus, Walker, Mon. Chal. i. 207.

a. England. From Mr. Walker's collection.

Urolepis, Walker.

Urolepis maritima, Cat. Chalc. B.M.p. 26. Pteromalus salinus? Von Heyden. Ent. Zeit. 1847.

b. England. From Mr. Walker's collection.

Cyrtogaster, Walker.

Cyrtogaster vulgaris, Cat. Chalc. B. M.p.26. Dicormus aquisgranensis, Foerster, Mon. Pterom. i. xxxvi.

c. Prussia. Presented by E. Doubleday, Esq.

d. Prussia. From Mr. Walker's collection.

Cyrtogaster Scotica, Walker, Ent. Mag. i. 382.

a. Scotland. From Mr. Walker's collection.

Cyrtogaster thoracica, Walker, Ent. Mag. i. 383.

a. England. From Mr. Walker's collection.

Cyrtogaster pusilla, Walker, Ent. Mag. i. 383.

a. England. From Mr. Walker's collection.

Cyrtogaster clavicornis, Walker, Ent. Mag. i. 383.

a. England. From Mr. Walker's collection.

Cyrtogaste r obscura, Walker, Ent. Mag. i. 383.

a. England. From Mr. Walker's collection.

Cyrtogaster tenuis, Walker, Ent. Mag. i. 383.

a. England. From Mr. Walker's collection.

Crrtogaster cingulipes, Walker, Ent. Mag. i. 384.

a. England. From Mr. Walker's collection.

Cyrtogaster Pœsos, Walker, MSS. See Appendix.

a. England. From Mr. Walker's collection. 
Tityros, Walker, MSS. See Appendix.

Trtyros Poreia, Walker, MSS. See Appendix.

a. England. From Mr. Walker's collection.

\section{Pachylarthrus, Westw.}

Pachylarthrus flavicornis. Phacostomus patellanus, Nees. Hym. Ich. aff. Mon. ii. 121.

a. From Mr. Walker's collection.

Pachylarthrus patellanus. Phacostomus similis, Foerster, Mon. Pterom. i. xxxii. 2. Pteromalus palpigerus, Zetterstedt, Ins. Lapp. 3.

a. Prussia. From Mr. Walker's collection.

Pachylarthrus Elyces, Walker, Mon. Chal. i. 195.

a. France. From Mr. Walker's collection.

\section{Dicyclus, Walker.}

Dicyclus brevicornis, Walker, Ent. Mag. i. 456.

a. England. From Mr. Walker's collection.

\section{Toxe uma, Walker.}

Toxeuma Ericæ, Cat. Chalc. B. M. p. 28.

b. Prussia. Presented by E. Doubleday, Esq.

Sphegigaster, Spinola.

Sphegigaster flavicornis, Cat. Chalc. B. M. p. 28. Chrysolampus coronatus, Foerster Mon. Pterom. i. 35, 5.

b. Prussia. Presented by E. Doubleday, Esq.

c. Prussia. From Mr. Walker's collection.

Sphegigaster clavicornis. Merismus clavicornis, Walker, Ent. Mag. i. 377.

a. England. From Mr. Walker's collection.

Sphegigaster rufipes. Merismus rufipes, Walker, Ent. Mag. i. 378 .

a. England. From Mr. Walker's collection.

Sphegigaster Agriope, Walker, MSS. See Appendix.

$a$. England. From Mr. Walker's collection.

Sphegigaster Lasthenes, Walker, MSS. See Appendix.

a. England. From Mr. Walker's collection. 


\section{Syntomopus, Walker.}

Srntomopus thoracicus, Walker, Ent. Mag. i. 372.

a. England. From Mr. Walker's collection.

Syntomopus Dirce, Walker. Miscogaster Dirce, Mon. Chal.

i. 195.

a. England. From Mr. Walker's collection.

Megorismus, Walker.

Megorismus Aon, Walker, Ann. Nat. Hist. 1848.

a. From Mr. Walker's collection.

Megorismus Nicææ Miscogaster Nicææ, Walker, Mon. Chal. i. 197.

a. England. Fṛom Mr. Walker's collection.

Corvna, Walker.

Coryna clavata, Cat. Chalc. B. M. p. 29.

b. Prussia. Presented by L. Doubleday, Esq.

Dipara, Walker.

Dipara petiolata, Walker, Ent. Mag. i. 373.

a. England. From Mr. Walker's collection.

Merisus, Walker.

Merisus splendidus, Walker, Ent. Mag. ii. 167.

a. France. From Mr. Walker's collection.

Micromelus, Walker.

Micromelus pyrrhogaster. Pteromalus mutilus, Foerster, Mon.

Pterom. i. xxvii. 254.

a. Prussia. Presented by E. Doubleday, Esq.

b. Prussia. From Mr. Walker's collection.

\section{Meraporus, Walker.}

Meraporus graminicola, Cat. Chalc. B. M. p. 35. Curtis, Journ. Roy. Agric. Soc. vii. 97. pl. P, fig. 19.

b. England. From Mr. Walker's collection.

\section{Lamprotatos, Walker.}

Lamprotatus maculipes. Miscogaster maculipes, Walker, Ent. Mag. i. 460. Mon. Chal. i. 282.

a. England. From Mr. Walker's collection. 
Lamprotatus æneus. Miscogaster æneus, Walker, Ent. Mag. i. 461. Mon. Chal. i. 285.

a. England. From Mr. Walker's collection.

Lamprotatus nitidipes. Miscogaster nitidipes, Walker, Ent. Mag. i. 462. Mon. Chal. i. 286.

a. England. From Mr. Walker's collection.

Lamprotatus ovatus. Miscogaster ovata, Walker. Ent. Mag. i. 462. Mon. Chal. i.287.

a. England. From Mr. Walker's collection.

Lamprotatus convexus. Miscogaster convexa, Walker, Ent. Mag. i. 463. Mon. Chal. i. 288.

$a$. England. From Mr. Walker's collection.

Lamprotatus apicalis. Miscogaster apicalis, Walker, Ent. Mag. i. 463. Mon. Chal. i. 288.

$a$. England. From Mr. Walker's collection.

Lamprotatus tristis. Miscogaster tristis, Walker, Ent. Mag. i. 463. Mon. Chal. i. 288.

a. England. From Mr. Walker's collection.

Lamprotatus costalis. Miscogaster costalis, Walker, Ent. Mag. i. 463. Mon. Chal. i. 290.

a. England. From Mr. Walker's collection.

Lamprotatus cyaneus. Miscogaster cyanea, Walker, Ent. Mag. i. 463. Mon. Chal. i. 290.

a. England. From Mr. Walker's collection.

Lamprotatus contiguus. Miscogaster contigua, Walker, Ent. Mag. i. 464. Mon. Chal. i. 291.

a. England. From Mr. Walker's collection.

Lamprotatus Rubrius, Walker, Ann. Nat. Hist. 1846, 111.

a. Scotland, From Mr. Walker's collection.

Lamprotatus Helenor, Walker, Ann. Nat. Hist. 1846, 111.

a. Scotland. From Mr. Walker's collection.

Lamprotatus Venilia, Walker, Ann. Nat. Hist. 1846, 113.

a. England. From Mr. Walker's collection.

Lamprotatus Phlegias, Walker, Ann. Nat. Hist. xiv. 332.

a. Alten, Finmark. From Mr. Walker's collection.

Lamprotatus Mazæus, Walker, Ann. Nat. Hist. xiv. 333.

a. Alten, Finmark. From Mr. Walker's collection.

Lamprotatus Brises, Walker, Ann. Nat. Hist. xiv. 333.

a. Alten, Finmark. From Mr. Walker's collection. 
Lamprotatus Cleta, Walker, Ann. Nat. Hist. xiv. 334. a. Alten, Finmark. From Mr. Walker's collection.

Lamprotatus Scœa, Walker, Ann. Nat. Hist, xiv. 335.

a. Alten, Finmark. From Mr. Walker's collection.

Lamprotatus Leucon, Walker, Ann. Nat. Hist. xiv. 335.

a. Alten, Finmark. From Mr. Walker's collection.

Lamprotatus Lesches, Walker, Ann. Nat. Hist. xiv. 336.

a. Alten, Finmark. From Mr. Walker's collection.

Lamprotatus Icelos, Walker, Ann. Nat. Hist. xiv. 337.

a. Alten, Finmark. From Mr. Walker's collection.

Lamprotatus Mallius, Walker, MSS. See Appendix.

a. England. From Mr. Walker's collection.

Lamprotatus Zipoetes, Walker, MSS. See Appendix.

a. England. From Mr. Walker's collection.

Lamprotatus OEbares, Walker, MSS. See Appendix.

a. England. From Mr. Walker's collection.

Lamprotatus Phylander, Walker, MSS. See Appendix.

a. England. From Mr. Walker's collection.

Lamprotatus Acarnas, Walker, MSS. See Appendix.

a. England. From Mr. Walker's collection.

Lamprotatus Acilius, Walker, MSS. See Appendix.

a. England. From Mr. Walker's collection.

Lamprotatus Amulius, Walker, MSS. See Appendix. $a$. England. From Mr. Walker's colleetion.

Lamprotatus Labaris, Walker, MSS. See Appendix.

a. England. From Mr. Walker's collection.

Lamprotatus Bolgius, Walker, MSS. See Appendix.

a. England. From Mr. Walker's collection.

Lamprotatus Methymna, Walker, MSS. See Appendix.

a. England. From Mr. Walker's collection.

Lamprotatus Pycnos, Walker, MSS. See Appendix.

$a$. England. From Mr. Walker's collection.

Lamprotatus Coretas, Walker, MSS. See Appendix.

$a$. England. From Mr. Walker's collection.

Seladerma, Walker.

Seladerma breve, Walker, Ent. Mag. ii. 296.

a. England. From Mr. Walker's collection. 
Seladerma Capreæ. Cynips Capreæ, Linn. Syst. Nat. ii. 919, 14. Faun. Suec. 1531. Vill. Ent. Linn. iii. 74, 14. Fabr. Syst. Ent. ii. Ent. Syst. ii. 102, 13. Syst. Piez. 146, 15. Pteromalus Salicis, Nees. Hym. Ich. aff. Mon. ii. 105, 20.

a. England. From Mr. Walker's collection.

$b$. Prussia. From Mr. Walker's collection.

Seladerma Saurus, Walker, Ann. Nat. Hist. xiv. 338. a. Alten, Finmark. From Mr. Walker's collection.

\section{Semiotus, Walker.}

Semiotus Tauriscus, Walker, MSS. See Appendix. a. England. From Mr. Walker's collection.

\section{Metastenus, Walker.}

Metastenus concinnus, Walker, Ent. Mag. ii. 302.

a. England. From Mr. Walker's collection.

\section{Pachyneuron, Walker.}

Pachyneuron Pruni, Foerster, MSS. Walker, Ann. Nat. Hist. 1848.

a. Prussia. Presented by E. Doubleday, Esq. .

b. Prussia. From Mr. Walker's collection.

Pachyneuron concolor, Pteromalus concolor? Foerster, Mon.

Pterom. i. xxvi. 236.

a. Prussia. Presented by E. Doubleday, Esq.

$b$. Prussia. From Mr. Walker's collection.

\section{Meraporus, Walker.}

Meraporus exiguus, Walker, Ent. Mag. ii. 301.

a. England. From Mr. Walker's collection.

Eunotus, Walker.

Eunotus cretaceus, Walker, Ent. Mag. ii. 298. The Entomologist, pl. A, fig. 4.

a. England. From Mr. Walker's collection.

Spaniopus, Walker.

Spaniopus dissimilis, Walker, Ent. Mag. i. 466. Mon. Chal. i. 292.

a. England. From Mr. Walker's collection. 


\section{Psilocera, Walker.}

Psilocera obscura, Walker, Ent. Mag. i. 374. Metopon atrum, Fem. Ent. Mag. ii. 303. The Entomologist, pl. B, fig. 3. Merismus obscurus? Blanchard, Goureau, Ann. Soc. Ent. Fr. 2me. Série, iv. 478, pl. 11,f. 3.

a. England. From Mr.Walker's collection.

\section{Pteromalus, Swederus.}

Pteromalus fasciiventris, Cat. Chalc. B. M. p. 36.

b. Prussia. Presented by E. Doubleday, Esq.

Pteromalus tibialis, Cat. Chalc. B. M. p. 36. P. sodalis, Foerster, Mon. Pterom. i. xi. 24.

b. Prussia. From Mr. Walker's collection.

Pteromalus dilectus, Cat. Chalc. B. M. p. 37.

b. Prussia. Presented by E. Doubleday, Esq.

c. Prussia. From Mr. Walker's collection.

Pteromalus catillus, Cat. Chalc. B. M. p. 40. P. multicarinatus, Foerster, Mon. Pterom. i. xv. 83.

b. Prussia. From Mr. Walker's collection.

Pteromalus bracteatus, Cat. Chalc. B. M.p. 40. P. chalcolampus, Foerster, Mon. Pterom. i. xri. 103.

b. Prussia. From Mr. Walker's collection.

Pteromalus herbidus, Cat. Chale. B. M. p. 40. P. dilectus, Foerster, Mon. Pterom. i. xxiv. 205.

b. Prussia. From Mr. Walker's collection.

Pteromalus affinis, Cat. Chalc. B. M. p.41. P. statutus, Foerster, Mon. Pterom. i. xviii. 126.

b. Prussia. From Mr. Walker's collection.

Preromalus fumipennis, Cat. Chalc. B. M. p.41. P. nubeculosus,

Foerster, Mon. Pterom. i. xxviii. 260.

b. Prussia. From Mr. Walker's collection.

Pteromalus microcerus, Cat. Chalc. B. M. p. 42. P. reconditus, Foerster, Mon. Pterom. i. xi. 25.

b. Prussia. Presented by E. Doubleday, Esq.

c. Prussia. From Mr. Walker's collection.

Pteromalus subniger, Cat. Chal. B. M. p. 42. P. subniger, Foerster, Mon. Pterom. i. xx. 150.

b. Prussia. From Mr. Walker's collection. 
Pteromalus muscarum, Cat. Chalc. $p$. 42. P. psittacinus, Foerster, Mon. Pterom. i. xv. 86. P. larvarum, Nees. Hym. Ich. aff. Mon. ii. 95, 3. P. Thessalus, Walker, Mon. Chal. i. 268. Diplolepis larvarum, Spin. Ins. Lig. Fasc. iii. 162, 16.

b. Prussia. Presented by E. Doubleday, Esq.

c. Prussia. From Mr. Walker's collection.

Pteromalus puparum, Cat. Chalc. B. M. p, 43.

d. Prussia. Presented by E. Doubleday, Esq.

$e$. Prussia. From Mr. Walker's collection.

Pteromalus mesochlorus, Cat. Chalc. B. M.p. 43. P. acuminatus,

Foerster, Mon. Pterom. i. xiv. 70.

b. Prussia. From Mr. Walker's collection.

Pteromalus micans, Cat. Chalc. B. M. p. 43. P. varians, Nees.

Hym. Ich. aff. Mon. ii. 101, 15.

c. Prussia. Presented by E. Doubleday, Esq.

Preromalus divisus, Cat. Chalc. B. M. p. 44.

$c$. Prussia. From Mr. Walker's collection.

Pteromalus hilaris, Cat. Chalc. B. M.p.45. P. quæsitus, Foerster, Mon. Pterom. i. xi. 30.

b. Prussia. From Mr. Walker's collection.

Pteromalus Mutia, Cat. Chalc. B. M. p. 46. P. vorax, Foerster, Mon. Pterom. i. xiv. 74.

b. Prussia. Presented by E. Doubleday, Esq.

c. Prussia. From Mr. Walker's collection.

Pteromalus Lucilla, Cat. Chalc. B. M. $p .47$. P. foveolatus,

Foerster, Mon. Pterom. i. xiii. 48.

b. Prussia. Presented by E. Doubleday, Esq.

c. Prussia. From Mr. Walker's collection.

Pteromalus terminalis. Platyterma terminale, Walker, Ent. Mag. ii. 306.

a. England. From Mr. Walker's collection.

Pteromalus remotus. Platyterma remotum, Walker, Ent. Mag. ii. 342.

a. England. From Mr. Walker's collection.

Pteromalus laticornis. Platyterma laticorne, Walker, Ent. Mag. ii. 304.

a. England. From Mr. Walker's collection.

Pteromalus fulvipennis. Amblymerus fulvipennis, Walker, Ent. Mag. ii. 344.

a. England. From Mr. Walker's collection. 
Pteromalus humilis. Amblymerus humilis, Walker, Ent. Mag. ii. 346.

a. England. From Mr. Walker's collection.

Pteromalus nitescens. Amblymerus nitescens, Walker, Ent. Mag. ii. 347.

a. Englaud. From Mr. Walker's collection.

Pteromalus hebes. Amblymerus hebes, Walker, Ent. Mag. ii. 348.

a. England. From Mr. Walker's collection.

Pteromalus tenellus. Amblymerus tenellus, Walker, Ent. Mag. ii. 348.

a. England. From Mr. Walker's collection.

Pteromalus fulvipes. Amblymerus fulvipes, Walker, Ent. Mag. ii. 348.

a. England. From Mr. Walker's collection.

Pteromalus nanus. Amblymerus nanus, Walker, Ent. Mag. ii. 349.

a. England. From Mr. Walker's collection.

Pteromalos iners. Amblymerus iners, Walker, Ent. Mag. ii. 50.

a. England. From Mr. Walker's collection.

Pteromalus trossulus. Amblymerus trossulus, Walker, Ent. Mag. ii. 350.

a. England. From Mr. Walker's collection.

Pteromalus tenebricus. Amblymerus tenebricus, Walker, Ent. Mag. ii. 351.

a. England. From Mr. Walker's collection.

Ptęromalus mirus. Amblymerus mirus, Walker, Ent. Mag ii. 350 .

a. England. From Mr. Walker's collection.

Pteromalus pygmæus. Eutelus pygmæus, Walker, Ent. Mag. a. England. From Mr. Walker's collection.

Pteromalus ocellus. Eutelus ocellus, Walker, Ent. Mag. ii. 359.

a. Scotland. From Mr. Walker's collection-

Pteromalus catenatus. Eutelus catenatus, Walker, Ent. May. ii. 362.

a. England. From Mr. Walker's collection.

Pteromalus helvipes. Eutelus helvipes, Walker, Ent. Mag. ii. 365.

a. England. From Mr. Walker's collection. 
Pteromalus politus. Eutelus politus, Walker, Ent. Mag. ii. 369. a. England. From Mr. Walker's collection.

Pteromalus vagans. Eutelus vagans, Walker, Ent. Mag. ii. 369.

a. England. From Mr. Walker's collection.

Pteromalus perversus, Walker, Ent. Mag. ii. 479.

a. England. From Mr. Walker's collection.

Pteromalus extentus, Walker, Ent. Mag. ii. 480.

a. England. From Mr. Walker's collection.

Pteromalus megachlorus, Walker, Ent. Mag. ii. 486.

a. England. From Mr. Walker's collection.

Pteromalus aurifer, Walker, Ent. Mag. ii. 487.

a. England. From Mr. Walker's collection.

Pteromalus apertus, Walker, Ent. May. ii. 489. P. chalcophanes, Foerster, Mon. Pterom. i. xxv. 220.

a. England. From Mr. Walker's collection.

b. Prussia. From Mr. Walker's collectiou.

Pteromalus cuprinus, Walker, Ent. Mag. ii. 489.

a. England. From Mr. Walker's collection.

Pteromalus curtus, Walker, Ent. Mag. ii.

a. England. From Mr. Walker's collection.

Pteromalus pinguis, Walker, Ent. Mag. ii. 490.

a. England. From Mr. Walker's collection.

Pteromalus despectus, Walker, Ent. Mag. ii. 491.

a. England. From Mr. Walker's collection.

Prenomalus purpureus, Walker, Ent. Mag. ii. 493.

a. England. From Mr. Walker's collection.

Pteromalus aniticus, Walker, Ent. Mag. ii. 494.

a. England. From Mr. Walker's collection.

Pteromalus rufinus, Walker, Ent. Mag. ii. 495.

a. England. From Mr. Walker's collection.

Pteromalus saturatus, Walker, Ent. Mag. ii. 495.

a. England. From Mr. Walker's collection.

Pteromalus futilis, Walker, Ent. Mag. ii. 496. P. operosus, Foerster, Mon. Pterom. i. xi. 28.

a. England. From Mr. Walker's collection.

b. Prussia. From Mr. Walker's collection.

Pteromalus ovatus, Nees. Hym. Ich.aff. Mon. ii. 103, 18.

a. Prussia. From Mr. Walker's collection. 
Pteromalus prætermissus, Foerster, Mon. Pterom. i. xvii. 107.

a. Prussia. From Mr. Walker's collection.

Pteromalus inclytus, Foerster, Mon. Pterom. i. xix. 145.

a. Prussia. From Mr. Walker's collection.

Pteromalus tibialis, Foerster, $M S S$.

a. Prussia. Presented by E. Doubleday, Esq.

Pteromalus laticeps, Foerster, MSS.

a. Prussia. Presented by E. Doubleday, Esq.

b. Prussia. From Mr. Walker's collection.

Pteromalus distinguendus, Foerster, Mon. Pterom. i. xv. 84.

a. Prussia. Presented by E. Doubleday, Esq.

$b$. Prussia. From Mr. Walker's collection.

Preromalus sulphuripes, Foerster, MSS.

a. Prussia. From Mr. Walker's collection.

Pteromalus sparsus, Foerster, Mon. Pterom. i. x. 18.

a. Prussia. Presented by E. Doubleday, Esq.

b. Prussia. From Mr. Walker's collection.

Pterumalus communis? Nees. Hym. Ich. aff. Mon. ii. 103, 17.

a. Prussia. Presented by E. Doubleday, Esq.

Pteromalus pexatus, Walker, Ent. Mag. ii. 499.

a. England. From Mr. Walker's collection.

Pteromalus detritus, Walker, Ent. Mag. ii. 499.

a. England. From Mr. Walker's collection.

Pteromalus latifrons, Walker, Ent. Mag. ii. 501.

a. England. From Mr. Walker's collection.

Pteromalus latipennis, Walker, Ent. Mag. iii. 96.

a. England. From Mr. Walker's collection.

Pteromalus mediocris, Walker, Ent. Mag. iii. 97.

a. England. From Mr. Walker's collection.

Pteromalus basalis, Walker, Ent. Mag. iii. 185.

a. England. From Mr. Walker's collection.

Pteromalos decisus, Walker, Ent. Mag. iii 185. P. opulentus, Foerster, Mon. Pterom. i. xxiv. 213.

a. England. From Mr. Walker's collection.

$b$. Prussia. From Mr. Walker's collection.

Pteromalus lautus, Walker, Ent. Mag. iii. 186.

a. England. From Mr. Walker's collection.

Pteromalus infectus, Walker, Ent. Mag. iii. 186.

a. England. From Mr. Walker's collection. 
Pteromalus impeditus, Walker, Ent. Mag. iii. 187.

a. England. From Mr. Walker's collection.

Pteromalus grandiclava, Walker, Ent. Mag. iii. 193.

a. England. From Mr. Walker's collection.

Pteromalus fucicola, Walker, Ent. Mag. iii. 194.

a. England. From Mr. Walker's collection.

Pteromalus plenus, Walker, Ent. Mag. iii. 199.

a. England. From Mr. Walker's collection.

Pteromalus solutus, Walker, Ent. Mag. iii. 199.

a. England. From Mr. Walker's collection.

Pteromalus berylli, Walker, Ent. Mag. iii. 199. P. crassus, Foerster. Mon. Pterom. i. xxv. 218.

a. England. From Mr. Walker's collection.

b. Prussia. From Mr. Walker's collection.

Pteromalos thoracicus, Walker, Ent. Mag. iii. 200.

a. England. From Mr. Walker's collection.

b. Prussia. From Mr. Walker's collection.

Pteromalus omnivorus, Walker, Ent. Mag. iii. 204.

a. England. From Mr. Walker's collection.

b. Prussia. From Mr. Walker's collection.

Pteromalus nigroæneus, Walker, Ent. Mag. iii. 206.

a. England. From Mr. Walker's collection.

Pteromalus cliens, Walker, Ent. Mag. iii. 469.

a. England. From Mr. Walker's collection.

Pteromalus illudens, Walker, Ent. Mag. iii. 470.

a. England. From Mr. Walker's collection.

Pteromalus gracilis, Walker, Ent. Mag. iii. 477.

a. England. From Mr. Walker's collection.

Pteromalus dorsalis, Walker, Ent. Mag. iii. 478.

a. England. From Mr. Walker's collection.

Pteromalus subquadratus, Walker, Ent. Mag. iii. 478.

a. England. From Mr. Walker's collection.

Pteromalus signatus, Wulker, Ent. Mag. iii. 479.

a. England. From Mr. Walker's collection.

Pteromalus servulus, Walker, Ent. Mag. iii. 481.

a. England. From Mr. Walker's collection.

Pteromalus rusticus, Walker, Ent. Mag. iii. 482.

a. England. From Mr. Walker's collection. 
Pteromalus diversus, Walker, Ent. Mag. iii. 483.

a. England. From Mr. Walker's collection.

Pteromalus thalassinus, Walker, Ent. Mag. iii. 485.

a. England. From Mr. Walker's collection.

Pte romalus confinis, Walker, Ent. Mag. iii. 486.

a. England. From Mr. Walker's collection.

Pteromalus exilis, Walker, Ent. Mag. iii. 487.

a. England. From Mr. Walker's collection.

Pteromalus chrysammos, Walker, Ent. Mag. iii. 487.

a. England. From Mr. Walker's collection.

Pteromalus pervasus, Walker, Ent. Mag. iii. 489.

a. England. From Mr. Walker's collection.

Pteromalus longulus, Walker, Ent. Mag. iii. 491.

a. England. From Mr. Walker's collection.

Pteromalus comes, Walker, Ent. Mag. iii. 492.

a. England. From Mr. Walker's collection.

Pteromalus gentilis, Walker, Ent. Mag. iii. 493.

a. England. From Mr. Walker's collection.

Pteromalus equestris, Walker, Ent. Mag. iii. 495.

a. England. From Mr. Walker's collection.

Pteromalus simulans, Walker, Ent. Mag. iii. 495.

a. England. From Mr. Walker's collection.

Pteromalus Empoclus, Walker, Mon. Chal. i. 214.

a. England. From Mr. Walker's collection.

Pteroma lus Docimus, Walker, Mon. Chal. i. 217.

a. England. From Mr. Walker's collection.

Pteromalus Pronax. Walker, Mon. Chal. i. 220.

a. England. From Mr. Walker's collection.

Pteromalus Samus, Walker, Mon. Chal. i. 221.

a. England. From Mr. Walker's collection.

Pteromalus Vibullius, Walker, Mon. Chal. i. 221.

a. England. From Mr. Walker's collection.

Pteromalus Calligetus, Walker, Mon. Chal. i. 222.

a. England. From Mr. Walker's collection.

Pteromalus Japis, Walker, Mon. Chal. i. 222.

a. England. From Mr. Walker's collection.

Pteromalus Carcinus, Walker, Mon. Chal. i. 229.

a. England. From Mr. Walker's collection. 
Pteromalus Gargasus, Walker, Mon. Chal. i. 229.

a. England. From Mr. Walker's collection.

Pteromalus Flavius; Walker, Mon. Chal. i. 230.

a. England. From Mr. Walker's collection.

Pteromalus Gonatas, Walker, Mon. Chal. i. 231.

a. England. From Mr. Walker's collection.

Pteromalus Druso, Walker, Mon. Chal. i. 232.

a. England. From Mr. Walker's collection.

Pteromalus Aper, Walker, Mon. Chal. i. 234.

a. England. From Mr. Walker's collection.

Pteronalus Tigasis, Walker, Mon. Chal. i. 232.

a. England. From Mr. Walker's collection.

Pteromalus Vala, Walker, Mon. Chal. i. 234.

a. England. From Mr. Walker's collection.

Pteromalus Styrus, Walker, Mon. Chal. i. 243.

a. England. From Mr. Walker's collection.

Pteromalus Ariovistus, Walker, Mon. Chal. i. 244.

$a$. England. From Mr. Walker's collection.

Pteromalus Boreus, Walker, Mon. Chal. i. 246.

a. England. From Mr. Walker's collection.

Pteromalus Hippo, Walker, Mon. Chal. i. 247.

a. England. From Mr. Walker's collection.

Pteromalus Sophax, Walker, Mon. Chal. i. 249.

a. England. From Mr. Walker's collection.

Pteromalus Faustina, Walker, Mon. Chal. i. 251.

$a$. England. From Mr. Walker's collection.

Pteromalus Tiburtus, Walker, Mon. Chal. i. 251.

a. England. From Mr. Walker's collection.

Pteromalus Abila, Walker, Mon. Chal. i. 252.

a. England. From Mr. Walker's collection.

Preromalus Automedon, Walker, Mon. Chal. i. 254.

a. England. From Mr. Walker's collection.

Pteromalus Tereus, Walker, Mon. Chal. i. 255.

a. England. From Mr. Walker's collection.

Pteromalus Nyctimus, Walker, Mon. Chal. i. 257.

a. England. From Mr. Walker's collection.

Pteromalus Crotopus, Walker, Mon. Chal. i. 258.

a. England. From Mr. Walker's collection. 
Pteromalus Dercyllus, Walker, Mon. Chal. i. 259.

a. England. From Mr. Walker's collection.

Pteromalus Archidemus, Walker, Mon. Chal. i. 259.

a. England. From Mr. Walker's collection.

Pteromalus Orsippus, Walker, Mon. Chal. i. 260.

a. England. From Mr. Walker's collection.

Pterromalus Aglaope, Walker, Mon. Chal. i. 261.

a. England. From Mr. Walker's collection.

Pteromalus Erasippus, Walker, Mon. Chal. i. 261.

a. Englaud. From Mr. Walker's collection.

Pteromalus Fontanus, Walker, Mon. Chal. i. 262.

a. England. From Mr. Walker's collection.

Pteromalus Favorinus, Walker, Mon. Chal. i. 263.

a. England: From Mr. Walker's collection.

Pteromalus Coryphe, Walker, Mon. C'hal. i. 266.

a. England. From Mr. Walker's collection.

Pteromalus Acrœa, Walker, Mon. Chal. i. 266.

a. England. From Mr. Walker's collection.

Preromalus Ormenus, Walker, Mon. Chal. i. 269.

a. England. From Mr. Walker's collection.

Pteromalus Sophron, Walker, Mon. Chal. i. 270.

a. England. From Mr. Walker's collection

Pteromalus Zelus, Walker, Mon. Chal. i. 270.

a. England. From Mr. Walker's collection.

Pteromalus Hecato, Walker, Mon. Chal. i. 271.

a. England. From Mr. Walker's collection.

Pteromalus Phyllus, Walker, Mon. Chal. i. 272.

a. England. From Mr. Walker's collection.

Pteromalus Cælius, Walker, Mon. Chal. i. 272.

a. England. From Mr. Walker's collection.

Pteromalus Promulus, Walker, Ann. Nat. Hist. iv. 232.

a. Scotland. From Mr. Walker's collection.

Pteromalus Varro, Walker, Ann. Nat. Hist. iv. 233

a. Scotland. From Mr. Walker's collection.

Pteromalus Rhinthon, Walker, Ann. Nat. Hist. xiv. 341.

a. Isle of Soröe, Finmark. From Mr. Walker's collection.

Pteromalus Musæus, Walker, Ann. Nat. Hist. xiv. 340.

a. Alten, Finmark. From Mr. Walker's collection. 
Pteromalus Mazaces, Walker, Ann. Nat. Hist. xiv. 341. a. Alten, Finmark. From Mr. Walker's collection.

Pteromalus Lausus, Walker, Ann. Nat. Hist. xix. 392, 1. a. St. John's Bluff, Florida. Presented by E. Doubleday, Esq. Pteromalus Cratylus, Walker, Ann. Nat. Hist. xix. 392, 2. a. St. John's Bluff, Florida. Presented by E. Doubleday, Esq. Pteromalus Cassotis, Walker, Ann. Nat. Hist. xix. 393, 3. a. St. John's Bluff, Florida. Presented by E. Doubleday, Esq. Pteromalus Euthymus, Walker, Ann. Nat. Hist. xix. 393, 4. a. St. John's Bluff, Florida. Presented by E. Doubleday, Esq. Pteromalus Dipsas, Walker, Ann. Nat. Hist. xix. 394, 5. a. St. John's Bluff, Florida. Presented by E. Doubleday, Esq.

Pteromalus Hermeas, Walker, Ann. Nat. Hist. xix. 394, 6. a. St. John's Bluff, Florida. Prrsented by E. Doubleday, Esq. Pteromalus Epicles, Walker, Ann. Nat. Hist. xix. 394, 7. a. St. John's Bluff, Florida. Presented by E. Doubleday, Esq.

Pteromalus Damo, Walker, Ann. Nat. Hist. xix. 395, 8. a. St. John's Bluff, Florida. Presented by E. Doubleday, Esq. Pteromalus Eax, Walker, Ann. Nat. Hist. xix. 395, 9. a. St. John's Bluff, Florida. Presented by E. Doubleday, Esq. Pteromalus Doryssus, Walker, Ann. Nat. Hist. xix. 395, 10. a. St. John's Bluff, Florida. Presented by E. Doubleday, Esq. Pteromalus Timæa, Walker, Ann. Nat. Hist. xix. 396, 11. a. St. John's Bluff, Florida. Presented by E. Doubleday, Esq. Pteromalus Orontas, Walker, Ann. Soc. Ent. xix. 396, 12. a. St. John's Bluff, Florida. Presented by E. Doubleday, Esq. Pteromalus Dymnus, Walker, Ann. Nat. Hist. xix. 397, 13. a. St. John's Bluff, Florida. Presented by E. Doubleday, Esq. Pteromalus Eryx, Walker, Ann. Nat. Hist. xix. 397, 14. a. St. John's Bluff, Florida. Presented by E. Doubleday, Esq. Pteromalus Hybreas, Walker, Ann. Nat. Hist. xix. 397, 15. a. St. John's Bluff, Florida. Presented by E. Doubleday, Esq. Pteromalus Eurypon, Walker, Ann. Nat. Hist. xix. 398, 16. a. St. John's Bluff, Florida. Presented by E. Doubleday, Esq. Pteromalus Acrotatus, Walker, Trans. Linn. Soc. xx. 153. a. Scotland. From Mr. Walker's collection.

Pteromalus Bubaris, Walker, Trans. Linn. Soc. xx. 154. a. Scotland. From Mr. Walker's collection. 
Pteromalus Ection, Walker, Trans. Linn. Soc. xx. 154.

a. Scotland. From Mr. Walker's collection.

Pteromalus Xanthe, Walker, Trans. Linn. Soc. xx. 155.

a. Scotland. From Mr. Walker's collection.

Pteromalus Aollius, Walker, Trans. Linn. Soc. xx. 156. a. North Wales. From Mr. Waiker's collection.

Pteromalus Antho, Walker, Trans. Linn. Soc. xx. 156.

a. England. From Mr. Walker's collection.

Pteromalus Learchus, Walker, Trans. Linn. Soc. xx. 157.

a. Scotland. From Mr. Walker's collection.

Pteromalus Autorides, Walker, Trans. Linn. Soc. xx. 157. a. England. From Mr. Walker's collection.

Pteromalus Saravus, Walker, Trans. Linn. Soc. xx. 157.

a. England. From Mr. Walker's collection.

Pteromalus Anaxenor, Walker, Trans. Linn. Soc. xx. 158.

a. England. From Mr. Walker's collection.

Pteromalus Tedanius, Walker, Trans. Linn. Soc. xx. 158. a. England. From Mr. Walker's collection.

Pteromalus Naubolus, Walker, Trans. Linn. Soc. xx. 159. P. larvarum? Nees. Hym. Ich. aff. Mon. ii. 94, 422. Diplolepis larvarum? Spin. Ins. Lig. Fasc. iii. 162, 16. Dipl.

Braconidis? Bouchè, Natur. Gesch. der. Ins. i. 171, 65.

a. England. From Mr. Walker's collection.

Pteromalos Aglaus, Walker, Trans. Linn. Soc. xx. 160.

a. England. From Mr. Walker's collection.

Pteromalus Urgo, Walker, Trans. Linn. Soc. xx. 160.

a. England. From Mr. Walker's collection.

Pteromalus Orinus, Walker, Trans. Linn. Soc. xx. 161.

a. England. From Mr. Walker's collection.

Pteromalos Eson, Walker, MSS. See Appendix.

a. Englaud. From Mr. Walker's collection.

Pteromalus Camma, Walker, MSS. See Appendix.

a. England. From Mr. Walker's collection.

Preromalus Phasis, Walker. See Appendix.

a. England. From Mr. Walker's collection.

Preromalus Cyamon. See Appendix.

a. England. From Mr. Walker's collection.

Pteromalus Hyloe, Walker. See Appendix.

a. England. From Mr. Walker's collection. 
Pteromalus Acco, Walker. See Appendix. a. England. From Mr. Walker's collection.

Pteromalus Tychon, Walker. See Appendix. a. England. From Mr. Walker's collection.

Pteromalus Cerycus, Walker. See Appendix. a. England. From Mr. Walker's collection.

Pteromalus Arestor, Walker. See Appendix. a. England. From Mr. Walker's collection.

Pteromalus Eulimene, Walker, MSS. See Appendix. a. England. From Mr. Walker's collection.

Pteromalus Dipænos, Walker, MSS. See Appendix. a. England. From Mr. Walker's collection.

Pteromalus Glautias, Walker, MSS. See Appendix. a. England. From Mr. Walker's collection.

Pteromalus Suia, Walker, MSS. See Appendix.

a. England. From Mr. Walker's collection.

Pteromalus Amnisos, Walker, MSS. See Appendix.

a. England. From Mr. Walker's collection.

Pteromalus Zagreus, Walker, MSS. See Appendix. a. England. From Mr. Walker's collection.

Pteromalus Opheltes, Walker, MSS. See Appendix. a. England. From Mr. Walker's collection.

Pteronalus Mese, Walker, MSS'. See Appendix.

a. England. From Mr. Walker's collection.

Pteromalus Alopius, Walker, MSS. See Appendix.

a. England. From Mr. Walker's collection.

Pteromalus Phylacis, Walker, MSS. See Appendix.

a. England. From Mr. Walker's collection.

Pteromalus Cæno, Walker, MSS. See Appendix.

a. England. From Mr. Walker's collection.

Pteromalus Corion, Walker, MSS. See Appendix.

a. England. From Mr. Walker's collection.

Pteromalus Hermachus, Walker, MSS. See Appendix.

a. England. From Mr. Walker's collection.

Pteromalus Temesa, Walker, MSS. See Appendix.

a. England. From Mr. Walker's collection.

Preromalus Drepanon, Walker, MSS. See Appendix.

๙. England. From Mr. Walker's collection. 
Preromalus Belesis, Walker, MSS. See Appendix.

a. England. From Mr. Walker's collection.

Pteromalus Lebadeia, Walker, MSS. See Appendix.

a. England. From Mr. Walker's collection.

Pteromalus Berycynthos, Walker, MSS. See Appendix.

a. England. From Mr. Walker's collection.

Pteromalus Lebene, Walker, MSS. See Appendix.

a. England. From Mr. Walker's collection.

Pteromalus Cercaphrus, Walker, MSS. See Appendix.

a. England. From Mr. Walker's collectton.

Pteromalus Cytœum, Walker, MSS. See Appendix.

a. England. From Mr. Walker's collection.

Pteromalus Lampe, Walker, MSS. See Appendix.

a. England. From Mr. Walker's collection.

Pteromalus Anchinoë, Walker, MSS. See Appendix.

a. England. From Mr. Walker's collection.

Pteromalus Gallonius, Walker, MSS. See Appendix.

a. England. From Mr. Walker's collection.

Pteromalus Eleuthera, Walker, MSS. See Appendix.

a. England. From Mr. Walker's collection.

Pteromalus Lyttus, Walker, MSS. See Appendix.

a. England. From Mr. Walker's collection.

Pteromalus Bienna, Walker, MSS. See Appendix.

a. England. From Mr. Walker's collection.

Pteromalus Patro, Walker, MSS. See Appendix.

a. England. From Mr. Walker's collection.

Preromalus Lissos, Walker, MSS. See Appendix.

a. England. From Mr. Walker's collection.

Pteromalus Morys, Walker, MSS. See Appendix.

a. England. From Mr. Walker's collection.

Pteromalus Elyros, Walker, MSS. See Appendix.

a. England. From Mr. Walker's collection.

Pteromalus Rhytium, Walker, MSS. See Appendix.

a. England. From Mr. Walker's collection.

Preromalus Myle, Walker, MSS. See Appendix.

a. England. From Mr. Walker's collection.

Pteromalus Loeta, Walker, MSS. See Appendix.

a. England. From Mr. Walker's collection. 
Preromalus Androhius, Walker, MSS. See Appendix. $a$. England. From Mr. Walker's collection. Pteromalus Inatos, Walker, MSS. See Appendix. a. England. From Mr. Walker's collection. Pteromalus Axos. Walker, MSS. See Appendix. a. England. From Mr. Walker's collection. Pteromalus Otos, Walker, MSS. See Appendix. a. England. From Mr. Walker's collection. Pteromalus Carma, Walker, MSS. See Appendix. a. England. From Mr. Walker's collection. Pteromalus Sybritia, Walker, MSS. See Appendix. a. England. From Mr. Walker's collection.

Pteromalus Mesapos, Walker, MSS. See Appendix. a. England. From Mr. Walker's collection.

Pteromalus Larymna, Walker, MSS. See Appendix. a. England. From Mr. Walker's collection.

Pteromalus Etearchus, Walker, MSS. See Appendix. a. England. From Mr. Walker's collection.

Pteromalus Phalasurna, Walker, MSS. See Appendix. a. England. From Mr. Walker's collection.

Pteromalus Themiso, Walker, MSS. See Appendix. a. England. From Mr. Walker's collection.

Preromalus Allutius, Walker, MSS. See Appendix. a. England. From Mr. Walker's collection. Pteromalus Priansos, Walker, MSS. See Appendix. a. England. From Mr. Walker's collection.

Pteromalus Gigon, Walker, MSS. See Appendix. a. England. From Mr. Walker's collection.

Pteromalus Orthagus, Walker, MSS. See Appendix. a. England. From Mr. Walker's collection.

Pteromalus Diomedon, Walker, MSS. See Appendix. a. England. From Mr. Walker's collection.

Pteromalus Alimentus, Walker, MSS. See Appendix. a. England. From Mr. Walker's collection.

Pteromalus Cœlius, Walker, MSS. See Appendix. a. England. From Mr. Walker's collection.

Pteromalus Æacus, Walker, MSS. See Appendix. a. England. From Mr. Walker's collection. 
Pteromalus Alope, Walker, MSS. See Appendix.

a. England. From Mr. Walker's collection.

Pteromalus Cepio, Walker, MSS. See Appendix.

a. England. From Mr. Walker's collection.

Pteromalus Hrtacina, Walker, MSS. See Appendix.

a. England. From Mr. Walker's collection.

Pteromalus Leuce, Walker, MSS. See Appendix.

a. England. From Mr. Walker's collection.

Pteromalus Hyrtacus, Walker, MSS. See Appendix.

a. England. From Mr. Walker's collection.

\section{Hetroxys, Westwood.}

Hetroxys invenustus. Pteromalus invenustus, Walker, Ent. Mag. iv. 11.

a. England. From Mr. Walker's collection.

Hetroxys macromerus. Pteromalus macromerus. Walker, Ent. Mag. iv. 11.

a. Englaud. From Mr. Walker's collection.

Hetroxys præpileus. Pteromalus præpileus, Walker, Ent. Maq. iv. 12.

a. England. From Mr. Walker's collection.

Hetroxys dimidiatus. Pteromalus dimidiatus, Walker, Ent. Mag. iv. 12.

a. England. From Mr. Walker's collection.

Hetroxys Aponius. Walker, MSS. See Appendix.

a. England. From Mr. Walker's collection.

\section{Cheiropachus, Westwood.}

Cheiropachus Colon. Sphex Colon, Linn. Syst. Nat. i. 2, 945, 28. Faun. Suec. 1656. Diplolepis quadrum, Fabr. Pteromalus quadrum, Zetterstedt, Ins. Lapp. 421. Cheiropachus quadrum, Cat. Chalc. B. M. p. 50.

c. Prussia. Presented by E. Doubleday, Esq.

d. Prussia. From Mr. Walker's collection.

Macroneura, Walker.

Macroneura maculipes, Cat. Chalc. B. M. p. 51.

b. Prussia. Presented by E. Doubleday, Esq. 
Trigonoderus, Westurood.

Trigonoderus tristis, Walker, Ent. Mag. iv. $1 \%$.

a. England. From Mr. Walker's collection.

Trigonoderus amabilis, Walker, Ent. Mag. iv. 20.

a. England. From Mr. Walker's collection.

Trigonoderus contemptus, Walker, Ent. Mag. iv. 22.

a. England. From Mr. Walker's collection.

Trigonoderus atrovirens, Walker, Ent. Mag. iv. 22.

a. England. From Mr. Walker's collection.

Trigonoderus Lappa, Walker, MSS. See Appendix.

a. England. From Mr. Walker's collection.

Trigonoderus Tarrha, Walker, MSS. See Appendix.

a. England. From Mr. Walker's collection.

Trigonoderus Alebion, Walker, MSS. See Appendix.

a. England. From Mr. Walker's collection.

Trigonoderus Polichna, Walker, MSS. See Appendix.

a. England. From Mr. Walker's collection.

\section{Platynocheilus, Westwood.}

Platynocherlus Erichsonii, Westwood, Ent. Mag. iii. 437. Stenocera Erichsonii, Walker, Mon. Chal. i. 276.

a. England. From Mr. Walker's collection.

Platynocheilus Derceto. Stenocera Derceto, Walker, Mon. Chal. i. 275.

a. England. From Mr. Walker's collection.

Stenocera, Walker.

Stenocera Walkeri, Curtis, Brit. Ent.596. Walker, Ent. Mag. iv. 357 .

a. England. From Mr. Walker's collection.

Notanisus, Walker.

Notanisus versicolor, Walker, Ent. Mag. iv. 352.

a. South of France. From Mr. Walker's collection.

Cleonymus, Latr.

Cleonymus obscurus, Walker, Ent. Mag. iv. 352.

a. England. From Mr. Walker's collection. 


\section{Eupelyus, Dalman.}

Eupelmus Degeeri, Cat. Chalc. B. M.p. 53.

d. Prussia. Presented by E. Doubleday, Esq.

Eupelmus Fonteia, Walker, Ann. Nat. Hist. xx. 19.

a. St. John's Bluff, Florida. Presented by E. Doubleday, Esq.

Eupelmus Lamachus, Walker, Ann. Nat. Hist. xx. 20.

a. St. John's Bluff, Florida. Presented by E. Doubleday, Esq.

Eupelmus, Epicaste, Walker, Ann. Nat. Hist. xx. 20.

a. St. John's Bluff, Florida. Presented by E. Doubleday, Esq.

Eupeluus Acron, Walker, MSS. See Appendix.

a. England. From Mr. Walker's collection. .

Eupelyus excavatus, Dalm. Kongl. Vet. Acad. Handl. 1820.

Nees. ab. Ess. Hym. Ich. aff. Mon. ii. 79. Walker, Ent. Mag.

iv. 362. Urocryptus excavatus, Westwood.

a. England. From Mr. Walker's collection.

\section{Ericy dnus, Haliday.}

Ericrdnus strigosus, Cat. Chalc. B. M. p. 53.

b. Prussia. Presented by E. Doubleday, Esq.

c. Prussia. From Mr. Walker's collection.

\section{Cheiloneurus, Westwood.}

Cheiloneurus elegans, Cat. Chalc. B. M. p. 54 .

a. Prussia. Presented by E. Doubleday, Esq.

\section{Metallon, Walker, MSS.}

Metallon Acacallis, Walker, MSS. See Appendix.

a. England. From Mr. Walker's collection.

\section{Cerchysius, Westwood.}

Cerchysius Vulso, Walker, Ann.Nat. Hist. xix. 114.

a. England. From Mr. Walker's collection.

Cerchysius Flaccus, Walker, Ann. Nat. Hist. xx. 21.

a. St. John's Bluff, Florida. Presented by E. Doubleday, Esq.

Encyrtus, Dalman.

Encyrtus cyaneus, Cat. Chalc. B. M.p. 54.

c. Prussia. Presented by E. Doubleday, Esq.

d. Prussia. From Mr. Walker's collection. 
Encyrtus Glapuyra, Cat. Chalc. B. M.p.55.

b. Prussia. Presented by E. Doubleday, Esq.

c. Prussia. From Mr. Walker's collection.

Encrrtus barbarus, Cat. Chalc. B. M. p.55.

b. Prussia. Presented by E. Doubleday, Esq.

c. Prussia. From Mr. Walker's collection.

Encyrtus subplanus, Cat. Chalc. B. M. p. 56. E. sceptriger,

Foerster, Mon. Pterom. i. xliv. 3.

b. Prussia. Presented hy E. Doubleday, Esq.

c. Prussia. From Mr. Walker's collection.

Encyrtus truncatellus, Cat. Chalc. B. M.p.56.

$d$. Prussia. From Mr. Walker's collection.

Encyrtus Swederi, Cat. Chalc. B. M. p. 56.

d. Prussia. Presented by E. Doubleday, Esq.

e. Prussia. From Mr. Walker's collection.

Encyrtus tessellatus, Cat. Chalc. B.M.p.57.

b. Prussia. Presented by E. Doubleday, Esq.

c. Prussia. From Mr. Walker's collection.

Encyrtus sericeus, Cat. Chalc. B. M. p. 57.

d. Prussia. Presented by E. Doubleday, Esq.

$e$. Prussia. From Mr. Walker's collection.

Encyrtus punctipes, Cat. Chalc. B. M. p. 57.

c. Prussia. From Mr. Walker's collection.

d. Prussia. From Mr. Walker's collection.

Encrrtus fuscicollis, Cat. Chalc. B. M.p. 58.

b. Prussia. Presented by E. Doubleday, Esq.

c. England. From Mr. Walker's collection.

Encyrtus atricollis, Cat. Chalc. B. M. p. 58. Ratzeburg, Forst.

Insekten, iii. $t .8, f .13$.

b. Prussia. Presented by E. Doubleday, Esq.

Encyrtus ineptus, Cat. Chalc. B. M. p. 59.

c. Prussia. From Mr. Walker's collection.

Encyrtus Marsus, Walker, Ent. Mag. iv. 444.

a. England. From Mr. Walker's collection.

Ency rtus Sipylus, Walker, Ent. Mag. iv. 445.

a. England. From Mr. Walker's collection.

Encyrtus Comara, Walker, Ent. Mag. iv. 446.

a. England. From Mr. Walker's collection.

Encyrtus Zarina, Haliday, Ent. Mag. iv. 447.

a. England. From Mr. Walker's collection. 
Ency RTUS æneiventris, Haliday, Ent. Mag. iv. 447.

a. Isle of Bute. From Mr. Walker's collection.

Ency RTus Jalysus, Walker, Ent. Mag. iv. 448.

a. England. From Mr. Walker's collection.

Ency Rtus Madyes, Walker, Ent. Mag. 448.

a. England. From Mr. Walker's collection.

Encrrtus Chœrilus, Walker, Ent. Mag. iv. 450.

a. England, From Mr. Walker's collection.

Ency rtus Gellius, Walker, Ent. Mag. iv. 454.

a. England. From Mr. Walker's collection.

Encr Rtus Mattinus, Walker, Ent. Mag. iv. 455.

a. England. From Mr. Walker's collection.

Evcy RTus Machæras, Walker, Ent. Mag. iv. 460. Ann. Nat. Hist. xiv. 185.

a. England. From Mr. Walker's collection.

Encyrtus Ancharus, Walker, Ent. Mag. v. 36.

a. England. From Mr. Walker's collection.

Encyrtus Atheas, Walker, Ent. Mag. v. 37.

a. England. From Mr. Walker's collection.

Ency rtus Dius, Walker, Ent. Mag. v. 39.

a. England. From Mr. Walker's collection.

Encyrtus Sosius, Walker, Ent. Mag. v. 39.

a. England. From Mr. Walker's collection.

Encyrtus Corybas, Walker, Ent. Mag. v. 40. a. England. From Mr. Walker's collection.

Encyrtus Sosares, Walker, Ent. Mag. v. 41. a. England. From Mr. Walker's collection.

Ency Rtus Pertiades, Walker, Ent. Mag. v. 42.

a. England. From Mr. Walker's collection.

Ency Rtus Thinæus, Walker, Ent. Mag. v. 42.

a. England. From Mr. Walker's collection.

Encyrtus Dercilus, Walker, Ent. Mag. v. 43.

a. England. From Mr. Walker's collection.

Encyrtus Babas, Walker, Ent. Mag. v. 44.

a. England. From Mr. Walker's collection.

Enctrtus Ariantes, Walker, Ent. Mag. v. 44.

a. England. From Mr. Walker's collection.

Encrrtus Elbasus, Walker, Ent. Mag. v. 45.

a. England. From Mr. Walker's collection. 
Encyrtus Scaurus, Walker. Ent. Mag. v. 45. a. England. From Mr. Walker's collection. Encyrtus Jancirus, Walker, Ent. Mag. v. 46. a. England. From Mr. Walker's collection. Ency RTus Sitalces, Walker, Ent. Mag. v. 47. a. England. From Mr. Walker's collection. b. Prussia. Presented by E. Doubleday, Esq. c. Prussia. From Mr. Walker's collection.

Ency rtus Tennes, Walker. Ent. Mag. v. 47. a. England. From Mr. Walker's collection.

Encyrtus cyanocephalus, Dalm. Kongl. Vet. Acad. Handl. 1820. a. Sweden. From Mr. Walker's collection.

Encyrtus cyanellus, Dalm. Kongl. Vet. Acad. Handl. 1820.

a. Sweden. From Mr. Walker's collection.

Encyrtus brevicornis, Dalm. Kongl. Vet. Acad. Handl. 1820.

a. Sweden. From Mr. Walker's collection.

Encrrtus melanacis, Dalm. Konyl. Vet. Acad. Handl. 1820.

a. Sweden. From Mr. Walker's collection.

Encrrtus lunatus, Dalm. Kongl. Vet. Acad. Handl. 1820.

a. Sweden. From Mr. Walker's collection.

Encyrtus longicornis, Dalm. Kongl. Vet. Acad. Handl. 1820.

a. Sweden. From Mr. Walker's collection.

Encyrtus Parus. Walker, Ent. Mag. v. 47.

a. England. From Mr. Walker's collection.

Encyrtus clavicornis, Dalm. Kongl. Vet. Acad. Handl. 1820. Nees. Hym. Ich. aff. Mon. ii. 225.

a. England. From Mr. Walker's collection.

b. Prussia. Presented by E. Doubleday, Esq.

c. Prussia. From Mr. Walker's collection.

Encyrtus Eupales, Walker, Ent. Mag. v. 51.

a. England. From Mr. Walker's collection.

Encyrtus clavellatus, Dalman, Kongl. Vet. Acad. Handl. 1820. $355,41$.

a. Prussia. Presented by E. Doubleday, Esq.

$b$. Prussia. From Mr. Walker's collection.

Encyrtus Arceanus, Walker, Ent.Mag. v. 52.

a. England. From Mr. Walker's collection.

Encyrtus Tanais, Walker, Ent. Mag. v. 52.

a. England. From Mr. Walker's collection. 
Encyrtus Dahlbomii, Westwood, Lond. and Edin. Phil. Mag. 3rd Series, x. 63, 441. Walker, Ent. Mag. v. 53.

a. England. From Mr. Walker's collection.

Encyrtus hederaceus, Westwond, Lond. and Edin. Phil, Mag. 3rd Series, x. 63, 441.

a. England. From Mr. Walker's collection.

Ency RTts Erylus, Walker, Ent. May. v. 518.

a. England. From Mr. Walker's collection.

Encyrtus Dores, Walker, Ent. Mag. v. 419.

a. England. From Mr. Walker's collection.

Encyrtus Amathus, Walker,Ent. Mag. v. 421.

a. England. From Mr. Walker's collection.

Encyrtus corniger, Haliday, Walker. Ent. Mag. v. 114.

a. France. From Mr. Walker's collection.

Encyrtus mirabilis, Walker, Ent. Mag. v. 114. Cerapterocerus mirabilis, Westwood, Mag. Nat. Hist. vi. 495. Encyrtus mirabilicornis, Foerster, Mon. Pterom. 1. xliii. 2. Encyrtus poduroides, Kollar.

a. England. From Mr. Walker's collection.

Encrrtus fulvescens, Walker, Ent. Mag. v. 115. Ectroma fulvescens, Westwood, Lond. and Edin. Phil. Mag. 3rd Series, 111, 344.

a. England. From Mr. Walker's collection.

Encyrtus Gahestus, Walker, Ent. Mag. v. 116.

a. Ireland. From Mr. Walker's collection.

Encyrtus Barca, Walker, Ent. Mag. v. 116.

a. England. From Mr. Walker's collection.

Ency rtus Caris, Walker, Ent. Mag. v. 422.

a. England. From Mr. Walker's collection.

Encyrtus Nadins, Walker. Ent. Mag. v. 423.

a. England. From Mr. Walker's collection.

Encyrtus Camirus, Walker, Ent. Mag. v. 425.

a. England. From Mr. Walker's collection.

Encyrtus Telesto, Walker, Ent. Mag. v. 425.

a. England. From Mr. Walker's collection.

Encyrtus Meon, Walker, Ent. Mag. v. 426.

a. England. From Mr. Walker's collection.

Ency rtus Epona, Walker, Ann. Nat. Hist. xiv. 184.

a. England. From Mr. Walker's collection. 
Encrrtus Pyttalus, Walker, Ann. Nat. Hist. xiv. 184. a. England. From Mr. Walker's collection.

Encrrtus Nicippe, Walker, Ann. Nat. Hist.iv. 234. a. England. From Mr. Walker's collection. Ency rtus Thyra, Walker, Ent. May. v. 427. a. France. From Mr. Walker's collection.

Encyrtus Obodas, Walker, Ent. May. v. 428.

a. France. From Mr. Walker's collection.

Encyrtus Baleus, Walker, Ent. Mag. v. 428.

a. France. From Mr. Walker's collection.

Evcrrtus Cedrenus, Walker, Ent. Mag. v. 112.

a. South of France. From Mr. Walker's collection.

Encyrtus Myrlea, Walker, Ent. Mag. v. 112.

a. South of France. From Mr. Walker's collection.

Encyrtus Arene, Walker, Ent. Mag. v. 429.

$a$. South of France. From Mr. Walker's collection.

Encyrtus Fadus, Waller, Ent. Mag. v. 429.

a. South of France. From Mr. Walker's collection.

Encŕrtus Sagillus, Walker, Ent. Mag. v. 430.

$a$. South of France. From Mr. Walker's collection.

Encyrtus Gastron, Walker, Ann. Nat. Hist. xx. 21.

a. St. John's Bluff, Florida. Presented by E. Doubleday, Esq.

Encyrtus Vectius, Walker, Ann. Nat. Hist.. xx. 21.

a. St. John's Bluff, Florida. Presented by E. Doubleday, Esq.

Ency rtus Reate, Walker, Ann. Nat. Hist. xx. 22.

a. St. John's Bluff, Florida. Presented by E. Doubleday, Esq.

Encyrtus Cervius, Walker, Ann. Nat. Hist. xvii. 177.

a. Scotland. From Mr. Walker's collection.

Encyrtus Meges, Walker, Ann. Nat. Hist. xvii. 178.

a. England. From Mr. Walker's collection.

Encyrtus Prœcia, Walker, Ann. Nat. Hist. xvii. 178.

a. England. From Mr. Walker's collection.

Encyrtus Peticus, Walker, Ann. Nat. Hist. xvii. 179.

a. England. From Mr. Walker's collection.

Encyrtus Metharma, Walker, Ann. Nat. Hist. xvii. 179.

a. England. From Mr. Walker's collection.

Ency rtus Genutius, Walker, Ann. Nat. Hist. xvii. 180.

a. England. From Mr. Walker's collection. 
Excyrtus Mamertus, Walker, Ann. Nat. Hist. xvii. 180.

a. Eugland. From Mr. Walker's collection.

Encyrtus Alycœus, Walker, MSS. See Appendix.

a. England. From Mr. Walker's collection.

Encyrtus Molns, Walker, MSS. See Appendix.

a. England. From Mr. Walker's collection.

Encyrtus Phrosime, Walker, MSS. See Appendix.

a. England. From Mr. Walker's collection.

Encyrtus Tylissos, Walker, MSS, See Appendix.

a. England. From Mr. Walker's collection.

Encrrtus Iophon, Walker, MSS. See Appendix.

a. England. From Mr. Walker's collection.

Eycyrtus Acratos, Walker, MSS. See Appendix.

a. England. From Mr. Walker's collection.

Encyrtus Hydramon, Walker, MSS. See Appendix.

a. England. From Mr. Walker's collection.

Encyrtus Philotis, Walker, MSS. See Appendix.

a. Hammeriest, Finmark. From Mr. Walker's collection.

Encyrtus Idmon, Walker, MSS. See Appendix.

a. Alten, Finmark. From Mr. Walker's collection.

Encyrtus Alcmon, Walker, MSS. See Appendix. E. ænescens?

Zetterstedt. Fauna Lapponica.

a. England. From Mr. Walker's collection.

Eincyrtus Genetyllis, Walker, MSS. See Appendix.

a. England. From Mr. Walker's collection.

Encyrtus Anthores, Walker, MSS. See Appendix.

a. Ireland. From Mr. Walker's collection.

Encyrtus Idya, Walker, MSS. See Appendix.

a. Alten, Finmark. From Mr. Walker's collection.

\section{Mrina, Nees.}

Mrina flava. Aphelinus flavus, Walker, Mon. Chal. i. 8. Eulophus flavus, Nees. Hym. Ich aff. Mon. ii. 167.

a. England. From Mr. Walker's collection.

Mrrna Argiope. Aphelinus Argiope, Walker, Mon. Chal. i. 9.

a. England. From Mr. Walker's collection. 
Myiva Idæus. Aphelinus Idæus, Walker, Mon. Chal. i. 12. a. England. From Mr. Walker's collection.

My rna Acœtes. Aphelinus Acœtes, Walker, Ann. Nat. Hist. xiv. 185.

a. England. From Mr. Walker's collection.

\section{Pteroptrix, Westwood.}

Pteroptrix Hemera, Walker, Mon. Chal. i. 14.

a. England. From Mr. Walker's collection.

Pteroptrix Janias, Walker, Mon. Chal. i. 17.

a. England. From Mr. Walker's collection.

Pteroptrix Acestes, Walker, Mon. Chal. i. 19.

a. England. From Mr. Walker's collection.

Pte roptrix Thione, Walker, Mon. Chal. i. 19.

a. England. From Mr. Walker's collection.

\section{Entedon, Dalman.}

Entedon viridis. E. Prodice, Walker, Cat. Chalc. B. M. p. 63.

Elachestus viridis, Nees. Hym. Ich. aff. Mon. ii. 138, 2.

$b$. Prussia. Presented by E. Doubleday, Esq.

c. Prussia. From Mr. Walker's collection.

Entedon Zanara, Cat. Chalc. B. M.p. 64.

$b$. Prussia. From Mr. Walker's collection.

Entedon cothurnatus. E. Argon, Walker, Cat. Chale. B. M.p.

64. Elachestus cothurnatus, Nees. Hym. Ich. aff. Mon. ii. $141,8$.

b. Prussia. Presented by E. Doubleday, Esq.

c. Prussia. From Mr. Walker's collection.

Entedon metallicus. E. Epigonus, Walker, Cat. Chalc. B. $M$.

Eulophus metallicus, Nees. Hym. Ich. aff. Mon. ii. 176, 34 .

b. Prussia. Presented by E. Doubleday, Esq.

c. Prussia. From Mr. Walker's collection.

Entedon turcicus, Walker, Mon. Chal.i.23. Eulophus turcicus,

Nees. Hym. Ich. aff. Mon. ii. 155, 5.

a. England. From Mr. Walker's collection.

Entedon Dimas, Walker, Mon. Chal. i. 24.

a. England. From Mr. Walker's collection.

Entedon Phænna, Walker, Mon. Chal. i. 25.

a. England. From Mr. Walker's collection. 
Entedon Damastes. Closterocerus Damastes, Walker, Ann. Nat. Hist. xx. 22.

a. St. John's Bluff, Florida. Presented by E. Doubleday, Esq.

Entedon Mento, Walker, Mon. Chal.i. 28.

a. England. From Mr. Walker's collection.

Entedon Tegar, Walker, Mon. Chal. i. 28.

a. England. From Mr. Walker's collection.

Entenon Coesius, Walker, Mon. Chal. i. 30.

a. England. From Mr. Walker's collection.

Entedon Alcetas. Derustenus Alcetas, Walker, Ann. Nat. Hist. xi. 31 .

a. England. From Mr. Walker's collection.

Entedon Butus, Walker, Mon. Chal. i. 32.

a. England. From Mr. Walker's collection.

Entedon Imbrasus, Walker, Ann. Nat. Hist. xx. 23.

a. St. John's Bluff, Florida. Presented by E. Doubleday, Esq.

Entedon Proclea, Walker, Mon. Chal. i. 33.

a. England. From Mr. Walker's collection.

Entedon Nautes, Walker, Mon. Chal. i. 33.

a. England. From Mr. Walker's collection.

Entedon Nephereus, Walker, Mon. Chal. i. 36.

a. England. From Mr. Walker's collection.

Entedon Amasis, Walker, Mon. Chal. i. 37.

a. England. From Mr. Walker's collection.

Eetedon Cilia, Walker, Mon. Chal. i. 37.

a. England. From Mr. Walker's collection.

Entedon Idyia, Walker, Mon. Chal. i. 39.

a. England. From Mr. Walker's collection.

Entedon Thonis, Walker, Mon. Chal.i. 44.

a. England. From Mr. Walker's collection.

Entedon Parsodes, Walker, Mon. Chal. i. 45.

a. England. From Mr. Walker's collection.

Entedon Phryne, Walker, Mon. Chal.i. 46.

a. England. From Mr. Walker's collection.

Entedon Melœnis, Walker, Mon. Chal.i.47.

a. England. From Mr. Walker's collection.

Entedon Thersamon, Walker, Mon. Chal.i. 48.

a. England. From Mr. Walker's collection. 
Entedon Ocyalus, Walker, Mon. Chal. i. 56. a. England. Erom Mr. Walker's collection. Entedon Pontinus, Walker, Mon. Chal. i. 60. a. England. From Mr. Walker's collection. Entedon Leucippus, Walker, Mon. Chal. i. 61. a. England. From Mr. Walker's collection.

Entedon Abrota, Walker, Mon. Chal. i. 62. a. England. From Mr. Walker's collection. Entedon Chilo, Walker, Mon. Chal. i. 63. a. England. From Mr. Walker's collection.

Entedon Alphenus, Walker, Mon. Chal. i. 64 . a. England. From Mr. Walker's collection.

Entedon Afranius, Walker, Mon. Chal. i. 65. a. England. From Mr. Walker's collection.

Entedon Aso, Walker, Mon. Chal. i. 66. a. England. From Mr. Walker's collection. Entedon Thoe, Walker, Mon. Chal. i. 68. a. England. From Mr. Walker's collection. Entedon Charaxus, Walker, Mon. Chal. i. 68. a. England. From Mr. Walker's collection. Entedon Tanis, Walker, Mon. Chal. i. 73. a. England. From Mr. Walker's collection.

Entedon Æsopus, Walker, Mon. Chal. i. 74. $a$. England. From Mr. Walker's collection.

Entedon Eropus, Walker, Mon. Chal. i. 75. a. England. From Mr. Walker's collection. Entedon Coilus, Walker, Mon. Chal. i. 79. a. England. From Mr. Walker's collection. Entedon Phruron, Walker, Mon. Chal. i. 87. a. England. From Mr. Walker's collection. Entedon Epaphus, Walker, Mon. Chal. i. 89. a. England. From Mr. Walker's collection. Entedon Rhesus, Walker, Mon. Chal. i. 88. a. England. From Mr. Walker's collection. Entedon Marica, Walker, Mon. Chal. i. 88. a. England. From Mr. Walker's collection. Entedon Phaola, Walker, Mon.Chal. i. 89. a. England. From Mr. Walker's collection. 
Entedon Metius, Walker, Mon. Chal. i. 90. a. England. From Mr. Walker's collection. Entedon Clymene, Walker, Mon. Chal. i. 91. a. England. From Mr. Walker's collection. Entedon Charino, Walker, Mon. Chal. i. 96. a. England. From Mr. Walker's collection. Entedon Termerus, Walker, Mon. Chal. i. 96. a. England. From Mr. Walker's collection. Entedon Abdera, Walker, Mon. Chal. i. 98. a. England. From Mr. Walker's collection. Enredon Syma, Walker, Mon. Chal. i. 98. a. England. From Mr. Walker's collection. Evredon Ergias, Walker, Mon. C'hal. i. 100. a. England. From Mr. Walker's collection. Entedon Methion. Eulophus caniculatus, Foerster, Mon. Pterom. i. xl. 18.

a. England. From Mr. Walker's collection.

b. Prussia. Presented by E. Doubleday, Esq.

c. Prussia. From Mr. Walker's collection.

Entedon Beon, Walker, Mon. Chal. i. 107. a. England. From Mr. Walker's collection. Entedon Acantha, Walker, Mon. Chal. i. 108. a. England. From Mr. Walker's collection. Entedon Carne, Walker, Mon. Chal. i. 123. a. England. From Mr. Walker's collection. Entedon Vagellius, Walker, Mon. Chal.i. 123. a. England. From Mr. Walker's collection. Entedon Erginnus, Walker, Mon. Chal.i. 124. a. England. From Mr. Walker's collection.

Entedon Hersilia, Walker, Ann. Nat. Hist.iv. 235.

a. England. From Mr. Walker's collection.

Entedon rutilans, Haliday, Walker, Ann. Nat. Hist. iv. 235. a. England. From Mr. Walker's collection. Entedon Acantha, Walker, Mon. Chal. i. 107. a. England. From Mr. Walker's collection. Extedon Epeus, Walker, Mon. Chal. i. 118. a. Eugland. From Mr. Walker's collection. Entedon Alaspharus, Walker, Mon. Chal.i. 109. a. England. From Mr. Walker's collection. 
Entedon Linus, Walker, Mon. Chal. i. 119. a. England. From Mr. Walker's collection.

Entedon Fufius, Walker, Ann. Nat. Hist. xvii. 182.

a. England. From Mr. Walker's collection.

Entedon Cœdicius, Walker, Ann. Nat. Hist. xvii. 183.

a. England. From Mr. Walker's collection.

Entedon Nautias, Walker, Ann. Nat. Hist. xvii. 183.

a. England. From Mr. Walker's collection.

Entedon Glabrio, Walker, Ann. Nat. Hist. xvii. 184.

a. England. From Mr. Walker's collection.

Entedon Clinus. Horismenus Clinus, Walker, Ann. Nat. Hist. xiv. 408.

a. Alten, Finmark. From Mr. Walker's collection:

Entedon Isander, Walker, MSS. See Appendix.

a. England. From Mr. Walker's collection.

Entedon Nurscia, Walker, MSS. See Appendix.

a. England. From Mr. Walker's collection.

Entedon Sauros, Walker, MSS. See Appendix.

a. England. From Mr. Walker's collection.

Entedon Axia, Walker, MSS. See Appendix.

a. England. From Mr. Walker's collection.

Entedon Antaradus, Walker, MSS. See Appendix.

a. England. From Mr. Walker's collection.

Entedon Erxias, Walker, MSS. See Appendix.

a. England. From Mr. Walker's collection.

Entedon Nænia, Walker, MSS. See Appendix.

a. England. From Mr. Walker's collection.

Entedon Stennos, Walker, MSS. See Appendix.

a. England. From Mr. Walker's collection.

Entedon Amadocus, Walker, MSS. See Appendix.

a. England. From Mr. Walker's collection.

Entedon Temena, Walker, MSS. See Appendix.

a. England. From Mr. Walker's collection.

Entedon Metagenes, Walker, MSS. See Appendix.

a. England. From Mr. Walker's collection.

Entedon Ergeteles, Walker, MSS. See Appendix.

a. England. From Mr. Walker's collection.

Entedon Adreus, Walker, MSS. See Appendix.

a. England. From Mr. Walker's collection. 
Entedon Icetas, Walker, MSS. See Appendix.

a. England. From Mr. Walker's collection.

\section{Epiclerus, Haliday.}

Epiclerus Panyas. Entedon Panyas, Walker, Mon. Chal. i. 120.

a. England. From Mr. Walker's collection.

Epiclerus Temenus. Entedon Temenus, Walker, Mon. Chal. i. 122.

e. England. From Mr. Walker's collection.

\section{Euderus, Haliday.}

Euderus Mithras, Walker, Mon. Chal. i. 93. a. England. From Mr. Walker's collection. Eude rus Herillus, Walker, Ann. Nat. Hist. xx. 23.

a. St. John's Bluff, Florida. Presented by E. Doubleday, Esq.

\section{Elachestus, Spinola.}

Elachestus Saon. Eulophus Saon, Walker, Mon. Chal. i. 175. a. England. From Mr. Walker's collection.

Elachestus Eurybates. Eulophus Eurybates, Walker, Mon. Chal. i. 178 .

a. England. From Mr. Walker's collection.

Elachestus Ticida. Eulophus Ticida, Walker, Mon. Chal. i. 179.

a. England. From Mr. Walker's collection.

Elachest us Levana, Walker, Ann. Nat. Hist. xx. 27.

a. St. John's Bluff, Florida. Presented by E. Doubleday, Esq.

\section{Eulophos, Geoffroy.}

Eulophus ramicornis, Cat. Chalc. B. M. p. 69.

a. Prussia. Preseuted by E. Doubleday, Esy.

b. Prussia. From Mr. Walker's collection.

Eulophus Gonippus, Cat. Chalc. B. M.p. 69.

a. Prussia. From Mr. Walker's collection. 
Eucophus gallarum, Nees. Hym. Ich. aff. Mon. ii. 170, 24. E. Euedoreschus, Walker, Cat. Chalc. B. M. p. 72. Ichneumon gallarum, Linn. Syst. Nat. ii. 939, 65. Faun. Suec. 16, 35. Vill. Ent. Linn. iii. 206, 232. Fabr. Syst. Ent. iii. Ent. Syst. ii. 186, 218. Diplolepis Gallarum, Fabr. Syst. Piez. 150, 8. Spin. Ins. Lig. Fasc. iv. 227. Pteromalus Gallarum, Latr. Gen. Crust. et Ins. iv. 31.

c. Prussia. Presented by E. Doubleday, Esq.

d. Prussia. From Mr. Walker's collection.

Eulophus Orsinus, Walker, Mon. Chal. i. 126.

a. England. From Mr. Walker's collection.

Eulophus Drupes, Walker, Mon. Chal. i. 127.

a. England. From Mr. Walker's collection.

Eulophus Anatole, Walker, Mon. Chal. i. 126.

a. England. From Mr. Walker's collection.

Eulophus Gobryas, Walker, Ann. Nat. Hist. xx. 26.

a. St. John's Bluff, Florida. Presented by E. Doubleday, Esq.

Eulophus Florus, Walker, Mon. Chal. i. 127.

a. England. From Mr. Walker's collection.

Eulophus Calavius, Walker, Ann. Nat. Hist. xx. 24.

a. St. John's Bluff, Florida. Presented by E. Doubleday, Esq.

Eu lophus Epulo, Walker, Mon. Chal. i. 141.

a. Eugland. From Mr. Walker's collection.

Eulophus Iphinoe, Walker, Ann. Nat. Hist. xx. 25.

a. St. John's Bluff, Florida. Presented by E. Doubleday, Esq.

Eulophus Eucritus, Walker, Mon. Chal.i. 142.

a. England. From Mr. Walker's collection.

Eulophos Cyriades, Walker, Ann. Nat. Hist. xx. 25.

a. St. John's Bluff, Florida. Presented by E. Doubleday, Esq.

Eulophus Minio, Walker, Ann. Nat. Hist. xx. 25.

a. St. John's Bluff, Florida. Presented by E. Doubleday, Esq.

Eulophus Leodamas, Walker, Mon. Chal. i. 130.

a. England. From Mr. Walker's collection.

Eulopнus Thespius, Walker, Mon. Chal. i. 127.

a. England. From Mr. Walker's collection.

Eulophus Sancus, Walker, Mon. Chal.i. 138.

a. England. From Mr. Walker's collection.

Eulophus Carbo, Walker, Mon. Chal. i. 138.

a. England. From Mr. Walker's collection. 
Eulophus Pronoe, Walker, Mon. Chal. i. 140. a. England. From Mr. Walker's collection. Eulophus Tidius, Walker, Mon. Chal. i. 146. a. England. From Mr. Walker's collection. Eu rophus Folia, Walker, Mon. Chal. i. 147. a. England. From Mr. Walker's collection. Eulophus Mania, Walker, Mon. Chal. i. 154. a. England. Frum Mr. Walker's collection. Eulophus Piscus, Walker, Mon. Chal. i. 155. a. England. From Mr. Walker's collection. Eulophus Drusilla, Walker, Mon. Chal. i. 156. a. England. From Mr. Walker's collection. Eulophus Euryalus, Haliday, Trans. Ent. Soc. iii. a. Ireland. From Mr. Walker's collection. Eulophus Apytus, Walker, Mon. Chal. i. 159. a. England. From Mr. Walker's collection. Eulophus Docilis, Walker, Mon. Chal. i. 159. a. England. From Mr. Walker's collection. Eulophus Prothenor, Walker, Mon. Chal. i. 180. a. England. From Mr. Walker's collection. Eulophus Attis, Walker, Mon. Chal. i. 181. a. England. From Mr. Walker's collection. Eulophus Sithon, Walker, Mon. Chal. i. 181. a. England. From Mr. Walker's collection. Euloperus Croton, Walker, Mon. Chal. i. 182. a. England. From Mr. Walker's collection. Eulophus Zeugma, Walker, Mon. Chal. i. 183. a. England. From Mr. Walker's collection. Eulophus Metanira, Walker, Mon. Chal. i. 183. a. England. From Mr. Walker's collection. Eulophus Cleopater, Walker, Mon. Chal. i. 184. a. England. From Mr. Walker's collection. Eulophus Diyllus, Walker, Mon. Chal. i. 185. a. England. From Mr. Walker's collertion. Eulophus Minyas, Walker, Ann. Nat. Hist. xx. 25. a. St. John's Bluff, Florida. Presented by E. Doubleday, Esq. Eulophus Ȧnaxippus, Walker, Ann. Nat. Hist. xvii. 182. a. England. From Mr. Walker's collection. 
Eulophvs Agathyllus, Walker, Zoologist. iv. 1458.

a. England. From Mr. Walker's collection.

Eulophus Idrieus, Walker, Ann. Nat. Hist. xiv. 409.

a. Alten, Finmark. From Mr. Walker's collection.

Eulophus Mamurius, Walker, MSS. See Appendix.

a. England. From Mr. Walker's collection.

Eulophus Rhamnius, Walker, MSS. See Appendix.

a. England. From Mr. Walker's collection.

Eulophus stygius, Boheman, MSS. See Appendix.

$a$. Sweden. From Mr. Walker's collection.

Eulophus Pythodorus, Walker, MSS. See Appendix.

a. England. From Mr. Walker's collection.

Eulophus Acalle, Walker, MSS. See Appendix.

a. England. From Mr. Walker's collection.

Eulophus Veturius, Walker, MSS. See Appendix.

a. England. From Mr. Walker's collection.

Eucophus Scyllis, Walker, MSS. See Appendix.

a. England. From Mr. Walker's collection.

Cirrospilus, Westwood.

Cirrosprlus Eunapius, Walker, Ann. Nat. Hist. xx. 27.

a. St. John's Bluff, Florida. Presented by E. Doubleday, Esq.

Cirrospilus flavovarius. Eulophus arcuatus, Foerster, Mon.

Pterom. i. xxxix. 8. E. 4-maculatus, Foerster, Mon. Pterom.

i. $x x x i x .6$.

a. Prussia. Presented by E. Doubleday, Esq.

b. Prussia. From Mr. Walker's collection.

Cirrospilus Pacuvius, Walker, Ann. Nat. Hist. i. 383.

a. England. From Mr. Walker's collection.

Cir rospilus Phorbas, Walker, Ann. Nat. Hist.i. 384.

a. England. From Mr. Walker's collection.

Cir rospilus Arsames, Walker, Ann. Nat. Hist. i. 384.

a. England. From Mr. Walker's collection.

Cirrospilus Orelia, Walker, Ann. Nat. Hist. i. 450.

a. England. From Mr. Walker's collection.

Cirrospilus Eudora, Walker, Ann. Nat. Hist. i. 452.

a. England From Mr. Walker's collection.

Cirrospilus Procles, Walker, Ann. Nat. Hist. i. 453.

a. England. From Mr. Walker's collection. 
Cir rospilus Abastor, Walker, Ann. Nat. Hist. i. 453.

a. England. From Mr. Walker's collection.

Cirrospilus Smilis, Walker, Mon. Chal. i. 317.

a. England. From Mr. Walker's collection.

Cirrospilus Myron, Walker, Mon. Chal. i. 294.

a. England. From Mr. Walker's collection.

Cirrospilus Deldon, Walker, Mon. Chal. i. 294.

a. England. From Mr. Walker's collection.

Cirrospilus Aratus, Walker, Ann. Nat. Hist. i. 453.

a. Ireland. From Mr. Walker's collection.

\section{Diglyphos, Haliday.}

Digly phus Poppæa, Haliday, MSS. See Appendix.

a. Ireland. From Mr. Walker's collection.

Diglyphus Aculen, Walker, MSS. See Appendix.

\section{Tetrastichus, Haliday.}

Tetrastichus Evonymellæ, Cat. Chalc. B. M.p. 73.

$b$. Prussia. Presented by E. Doubleday, Esq.

c. Prussia. From Mr. Walker's collection.

Tetrastichus Armæus, Cat. Chalc. B. $M$. p. 74. Eulophus setiseries, Foerster, Mon. Pterom. i. xxxix. 2.

b. Prussia. Presented by E. Doubleday, Esq.

c. Prussia. From Mr. Walker's collection.

Tetrastichus miser. T. Attalus, Walker, Cat. Chalc. B. M.p. 74.

Eulophus miser, Nees. Hym. Ich. aff. Mon. ii. 173, 28.

c. Prussia. Presented by E. Doubleday, Esq.

d. Prussia. From Mr. Walker's collection.

Tetrastichus Prosymna, Cat. Chalc. B. M. p. 75. Eulophus crinicornis? Perris. Ann. Soc. Ent. France. ix.

Tetrastichus Quercens, Cat. Chalc. B. M.p. 76.

b. Prussia. Presented by E. Doubleday, Esq.

c. Prussia. From Mr. Walker's collection.

Tetrastichus Rapo, Cat. Chalc. B. M. Diplolepis Microgastri, Bouché, Naturgesch. Ins. i. 168, 61. Pteromalus Microgasteris, Nees. Hym. Ich. aff. Mon. ii 423, 17. b.

b. Prussia. Presented by E. Doubleday, Esq.

c. Prussia. From Mr. Walker's collection. 
Tetrastichus Nerio, Cat. Chalc. B. M. p. 77. Eulophus Ericæ? Dufour, Ann. Soc. Ent. vii.

Tetrastichus Leucone, Cat. Chalc. B. M.p. 79. Eulophus longicaudatus, Foerster, Mon. Pterom. i. xl. 19.

c. Prussia. Presented by E. Doubleday, Esq.

$d$. Prussia. From Mr. Walker's collection.

Tetrastichus Metra. Cirrospilus Metra, Walker, Ann. Nat. Hist. ii. 201.

a. England. From Mr. Walker's collection.

Tetrastichus Euedochus. Cirrospilus Euedochus, Walker, Ann. Nat. Hist. ii. 204.

a. England. From Mr. Walker's collection.

Tetrastichus Cyrrhus. Cirrospilus Cyrrhus, Walker, Ann. Nat. Hist. ii, 350 .

a. England. From Mr. Walker's collection.

Tetrastichus æneus, Haliday, MSS. Ann. Nat. Hist. 1848.

a. Ireland. From Mr. Walker's collection.

Tetrastichus Mycerinus. Cirrospilus Mycerinus, Walker, Ann. Nat. Hist. ii. 350.

a. England. From Mr. Walker's collection.

Tetrastichus Tachos. Cirrospilus Tachos, Walker, Ann. Nat. Hist. ii. 352.

a. England. From Mr. Walker's collection.

Tetrastichus Tulis. Cirrospilus Tulis, Walker, Ann. Nat. Hist. ii. 354 .

a. England. From Mr. Walker's collection.

Tetrastichus Murcia. Cirrospilus Murcia, Walker, Ann. Nat. Hist. iii. 177.

a. England. From Mr. Walker's collection.

Tetrastichus Abantidas. Cirrospilus Abantidas, Walker, Ann. Nat. Hist. iii. 179.

a. England. From Mr. Walker's collection.

Tetrastichus Euagoras. Cirrospilus Euagoras, Walker, Ann. Nat. Hist. iii. 179.

a. England. From Mr. Walker's collection.

Tetrastichus Chares. Cirrospilus Chares, Walker, Ann. Nat. Hist. iii. 180.

a. England. From Mr. Walker's collection. 
Tetrastichus Beroe. Cirrospilus Beroe, Wulker, Ann. Nat. Hist. iii. 181.

a. England. From Mr. Walker's collection

Tetrastichus Rhacius, Walker, Ann. Nat. Hist. iii. 181.

a. England. From Mr. Walker's collection.

Tetrasticius Ëga. Cirrospilus $\mathbb{E}$ ga, Walker, Ann. Nat. Hist. iii. 181.

a. England. From Mr. Walker's collection.

Tetrasrichus Anticlea. Cirrọspilus Anticlea, Walker, Ann. Nat. Hist. iii. 182.

a. England. From Mr. Walker's collection.

Tetrastichus Sotades. Cirrospilus Sotadés, Walker, Ann. Nut. Hist. iii. 417.

a. England. From Mr. Walker's collection

Tetrastichus Anyta. Cirrospilus Anyta, Walker, Ann. Nat. Hist. iii. 417.

a. England. From Mr. Walker's collection.

Tetrasticuus Zenocia. Cirrospilus Zenocia, Walker, Ann. Nut. Hist. iii. 418.

a. England. From Mr. Walker's collection.

Tetrastichus Zopyrus. Cirrospilus Zopyrus, Walker, Ann. Nut. Hist. iv. 29.

a. England. From Mr. Walker's collection.

Tetrastichus Arathis. Cirrospilus Arathis, Walker, Ann. Nat. Hist. iv. 29.

a. England. From Mr. Walker's collection.

Tetrastichus Rhode. Cirrospilus Rhode, Walker, Ann. Nat. Hist. iv. 29.

a. England. From Mr. Walker's collection.

Tetrastichus Clito. Cirrospilus Clito, Walker, Ann. Nat. Hist. iv. 30 .

a. England. From Mr. Walker's collection.

Tetrastichus Rhosaces. Cirruspilus Rhosaces, Walker, Mon. Chal. i. 293.

a. England. From Mr. Walker's collection.

Tetrastichus Myron. Cirrospilus Myron, Walker, Mon. Chal. i. 294

a. England. From Mr. Walker's collection. 
Tetrastichus Deldon. Cirrospilus Deldon, Walker, Mon. Chat. i. 294.

a. England. From Mr. Walker's collection.

Tetrastichus Lycidas. Cirrospilus Lycidas, Walker, Mon. Chal. i. 295.

a. England. From Mr. Walker's collection.

Tetrastichus Sinope. Cirrospilus Sinope, Walker, Mon. Chal. i. 295.

a. England. From Mr. Walker's collection.

Tetrastichus Myrsus. Cirrospilus Myrsus, Walker, Mon. Chal. i. 296.

a. England. From Mr. Walker's collection.

Tetrastichus Conon. Cirrospilus Conon, Walker, Mon. Chal. i. 296.

a. England. From Mr. Walker's collection.

Tetrastichus Voranus. Cirrospilus Voranus, Walker, Mon. Chal. i. 298.

a. England. From Mr. Walker's collection.

Tetrastichus Simo. Cirrospilus Simo, Walker, Mon. Chal. i. 298.

a. England. From Mr. Walker's collection.

Tetrastichus Plangon. Cirrospilus Plangon, Walker, Mon. Chal. i. 298.

a. England. From Mr. Walker's collection.

Tetrastichus Vaccus. Cirrospilus Vaccus, Walker, Mon. Chal. i. 299.

a. England. From Mr.Walker's collection.

'Tetrastichus Oxathres. Cirrospilus Oxathres, Walker, Mon. Chal. i. 299.

a. England. From Mr. Walker's collection.

Tetrastichus Eleuchia. Cirrospilus Eleuchia, Walker, Mon. Chal. i. 301 .

a. England. From Mr. Walker's collection.

Tetrastichus Dotus. Cirrospilus Dotus, Walker, Mon. Chal. i. 301 .

a. England. From Mr. Walker's collection.

Tetrastichus Xeuxes. Cirrospilus Xeuxes, Walker, Mon. Chal. i. 301 .

a. England. From Mr. Walker's collection. 
Tetrastichus Ione. Cirrospilus Ione, Walker, Mon. Chal. i. 301.

a. England. From Mr. Walker's collection.

Tetrastichus Vacuna. Cirrospilus Vacuna, Walker, Mon. Chal. i. 305 .

a. England. From Mr. Walker's collection.

Tetrastichus Oropus. Cirrospilus Oropus, Walker, Mon. Chal. i. 305.

a. England. From Mr. Walker's collection.

Tetrastrichus Agrus. Cirrospilus Agrus, Walker, Mon. Chal. i. 306.

a. England. From Mr. Walker's collection.

Temrastichus Tyrtœus. Cirrospilus Tyrtœus, Walker, Mon. Chal. i. 306.

a. England. From Mr. Walker's collection.

Tetrastichus Vicellius. Cirrospilus Vicellius, Walker, Mon. Chal. i. 307.

a. England. From Mr. Walker's collection.

Tetrastichus Epidius, Walker, Ann. Nat. Hist. xx. 28.

a. St. Johu's Bluff, Florida. Presented by E. Doubleday, Esq.

Tetrastichus Lyridice. Cirrospilus Lyridice, Walker, Mon. Chal. i. 307.

a. England. From Mr. Walker's eollection.

Tetrastichus Gaus. Cirrospilus Gaus, Walker, Mon. Chal. i. 308.

a. England. From Mr. Walker's collection.

Tetrastichus Lachares. Cirrospilus Lachares, Walker, Mon. Chal. i. 309.

a. England. From Mr. Walker's collection.

Tetrastichus Apama. Cirrospilus Apama, Walker, Mon. Chal. i. 310.

a. England. From Mr. Walker's collection.

Tetrastichus Faucula. Cirrospilus Faucula, Walker, Mon. Chal. i. 310 .

a. England. From Mr. Walker's collection.

Tetrastichus Racilla. Cirrospilus Racilla, Walker, Mon. Chal. i. 312.

a. England. From Mr. Walker's collection.

Tetrastichus Pamyles. Cirrospilus Pamyles, Walker, Mon. Chal. i. 313.

a. England. From Mr. Walker's collection. 
Tetrastichus Hypsistus. Cirrospilus Hypsistus, Walker, Mon. Chal. i. 316.

a. England. From Mr. Walker's collection.

Tetrastichus Smilis. Cirrospilus Smilis, Walker, Mon. Chal. i. 317.

a. England. From Mr. Walker's collection.

Tetrastichus Vincius. Cirrospilus Vincius, Walker, Mon. Chal. i. 317.

a. England. From Mr. Walker's collection.

Tetrastichus Eratus. Cirrospilus Eratus, Walker, Mon. Chal. i. 318.

a. England. From Mr. Walker's collection.

Tetrastichus Leocrates. Cirrospilus Leocrates, Walker, Mom. Chal. i. 319.

a. England. From Mr. Walker's collection.

Tetrastichus Totis. Cirrospilus Totis, Walker, Mon. Chal. i. 320.

a. England. From Mr. Walker's collection.

Tetrastichus Natras. Cirrospilus Natras, Walker, Mon. Chal. i. 320.

a. England. From Mr. Walker's collection.

Tetrastichus Hæmon, Walker, Ann. Nat. Hist. xx. 28.

a. St. John's Bluff, Florida. Presented by E. Doublerlay, Esq.

Tetrastichus Numeria. Cirrospilus Numeria, Walker, Mon. Chal. i. 321 .

a. England. From Mr. Walker's collection.

'Tetrastichus Anodaphus. Cirrospilus Anodaphus, Walker, Mon. Chal. i. 321.

a. England. From Mr. Walker's collection.

Tetrastichus Mutilia. Cirrospilus Mutilia, Walker, Mon. Chal. i. 322.

a. England. From Mr. Walker's collection.

Tetrastichus Gala, Walker, Ann. Nat. Hist.xx. 28.

$a$. St. John's Bluff, Florida. Presented by E. Doubleday, Esq.

Tetrastichus Trabea, Walker, Mon. Chal i. 323.

a. England. From Mr. Walker's collection.

Tetrastichus Suevius. Cirrospilus Suevius, Walker, Mon. C'hal. i. 323 .

a. England. From Mr. Walker's collection. 
Tetrastichus Rhœo. Cirrospilus Rhœo, Walker, Ann. Nat. Hist. iii. 417.

a. England. From Mr. Walker's collection.

Tetrastichus Teridae. Cirrospilus Teridae, Walker, Ann. Nat. Hist. iv. 236.

a. England. From Mr. Walker's collection.

Tetrastichus Biunchus. Cirrospilus Brunchus, Walker, Ann. Nat. Hist. iv. 236.

a. Scotland. From Mr. Walker's collection.

Tetrastichus Idothea, Walker, Ann. Nat. Hist. xiv. 409.

a. Alten, Finmark. From Mr. Walker's collection.

Tetrastichus Pausiris. Cirrospilus Pausiris, Walker, Mon. Chal. i. 327 .

a. England. From Mr. Walker's collection.

Tetrastichus Marbis. Cirrospilus Marbis, Walker, Mon. Chal. i. 330 .

a. England. From Mr. Walker's collection.

Tetrastichus Abydenus, Walker, MSS. See Appendix.

a. England. From Mr. Walker's collection.

Tetrastichus Bermius, Walker, MSS. See Appendix.

a. England. From Mr. Walker's collection.

Tetrastichus Axia, Walker, MSS. See Appendix.

a. England. From Mr. Walker's collection.

Tetrastichus Triarius, Walker, MSS. See Appendix.

a. England. From Mr. Walker's collection. 


\section{APPENDIX.}

\section{DESCRIPTIONS OF NEW SPECIES}

COMMUNICATED BY

FRANCIS WALKER, EsQ., F.L.S., \&c.

Eurytoma Cestius. Fem. Nigra, gracilis, genubus, tibiis apice tarsis, propedibusque rufis, alis limpidis. Page 100.

The slender body of this species easily distinguishes it from all other described Eurytomæ; E. atrum comes nearest to it in this respect, but is very different in structure. Black, narrow, linear, convex: head and chest or thorax thickly punctured, not shining: head a little broader than the chest : feelers or antennæ subclavate, slender, shorter than the chest; club spindle-shaped, more than twice the length of the preceding joint: fore-chest or prothorax large; its breadth slightly exceeding its length : hind-chest or metathorax well developed, obconic, declining, having a large furrow down the middle: petiole short: abdomen spindle-shaped, smooth, shining, keeled beneath, a little longer and narrower than the chest; metapodeon and octoon of moderate size; ennaton shorter; decaton much longer; protelum, paratelum, and telum of moderate size : legs black; knees, feet or tarsi, and tips of shanks or tibiæ red; fore-legs nearly all red: wings white; veins tawny; ulna rather thick, about one-third of the length of the humerus; radius shorter than the ulna. Length of the body $1 \frac{1}{4}$ line; of the wings 2 lines.

Page 100. Eurytoma Mucianus. Mas. Nigra, genubus, tarsis propedibusque fulvis, alis limpidis.

It resembles E. platyptera, but is distinguished from that species by its more nodose feelers. Black, convex : head and chest thickly punctured : feelers black, beset with whorls of hair, piceous 
towards the tips, rather longer than the chest : brearth of the forechest about twice its length: petiole rather long: abdomen gibbous, very much shorter than the chest; its height is somewhat less than its length: legs black; knees and feet tawny, as are also the whole of the fore-legs except the base of the thighs : wings colvurless; veins tawny; ulna not very broad, about one-fifth of the length of the humerus; radius longer than the ulna, a character wherein this Eurytoma differs from all the other British species, with the exception of E. plumata and rufipes. Length of the body 1 line; of the wings $1 \frac{1}{2}$ line.

Callimome Solinus. Mas. Viridis, abdomine purpureo, antennis nigris, tibiis piceis, tarsis fulvis, alis subfuscis. Page 103.

Allied to C. nigricornis, but haring a more slender body, with narrower and darker wings. Dark green, rather long and narrow : eyes dark red: feelers black, about half the length of the body, nearly filiform, moderately thick; first joint green: abdomen purple, rather narrower and shorter than the chest: hips, or coxæ, and thighs green; shanks piceous; knees, feet, and tips of the shanks tawny: wings narrow, and slightly tinged with brown; veins black; ulna nearly as long as the humerus; radius not more than one-third of the length of the ulna; cubitus less than one-half the length of the radius. Length of the body $1 \frac{1}{2}$ lines; of the wings 3 lines.

\section{Elatus, Walker, (n.g.) Page 104.}

This genus has a peculiar form, and somewhat resembles Perilampus and Callimome. Male. Body convex, arched: head and chest shagreened: head large, broader than the chest: feelers broad, subclavate, inserted in the middle of the front, much shorter than the chest; first joint long, slender ; second cup-shaped ; third and fourth very minute; tifth and following joints to the tenth short, broad, of nearly the same size, their breadth about twice their length; club conical, more than twice the length of the tenth joint : chest almost gibbous : fore-chest subquadrate, of moderate size; its breadth about twice its length: shield or scutum of the middle chest broad; sutures of the parapsides strongly marked; scutcheon or scutellum large, almost conical : hind-chest large, obconical, declining: petiole rither stout, shorter than the hind-chest: abdomen shorter and much narrower than the chest, smooth, shining, nearly elliptical, very convex, and forming a transverse ridge at the tip of 
the metapodeon which extends orer half the back; octoon also very large, and occupying the other half of the back, so that the following segments are not visible : ulna a little shorter than the humerus ; radius about one-fourth of the length of the ulna; culitus a little shorter than the radius, and hardly longer than in Callimome and Perilampus.

Elatus Thenæ. Cyaneo-viridis, abdomine aneo-viridi, antennis nigris, genubus tarsisque fuscis, tibiis apice fulvis, alis limpidis. Page 104.

Bluish green : eyes red : feelers black; first joint green : abdomen tinged with bronze: legs green; tips of shanks tawny; knees and feet brown: wings colourless, of moderate size; veins brown; wing-brand or stigma, very small. Length of the body 1 line; of the wings $1 \frac{3}{4}$ line.

\section{Tetramesa, Walker, (n. g.) Page 104.}

Female. Conrex: head and chest punctured, dull: head broader than the chest: feelers clavate, rather hairy, 13-jointed, as long as the chest; first joint long and slender; second nearly cupshaped; third and fourth very small; fifth and following joints to the tenth slightly cup-shaped, successively increasing in breadth and decreasing in length; club 3 -jointed, conical, full twice the length of the tenth joint: brealth of the fore-chest rather less than twice its leugth: sutures of the parapsides of the middle-chest strongly marked; axillæ large, separater from each other by a small interval : hind-chest obconical, declining, well developed: petiole short: abrlomen nearly elliptical, smooth, shining, deeply keeled beneath, elevated at the tip, shorter but hardly narrower than the chest; metapodeon very large ; octoon rery short; ennaton long; decaton and following segments very short : wings rather narrow; ulna about one-fourth of the length of the humerus; rarlius shorter than the ulna; cubitus as long as the radius; wing-brand very small.

Tetramesa Iarbas. Fem. Nigra, genubus tarsisque rufis, alis subfuscis. Page 104.

Black: knees and feet red; tips of the latter brown: wings slightly brown; veins piceous. Length of the body 1 line; of the wings $1 \frac{3}{4}$ line. 
Gastrancistrus longicornis. Fem. Nigro-viridis, antennis pedibusque piceis, genubus, tarsis tibiisque apice fulvis, alis limpidis. Page 105.

Very dark green, almost black: head and chest very finely shagreened: head a little broader than the chest: eyes dark red: feelers clavate, slender, piceous, longer than the chest ; first joint long and slender; second slightly cup-shaped; third and following joints to the seventh linear, nearly equal in size; club spindleshaped, rather broader than the seventh joint and more than twice its length: chest oval, rather flat: fore-chest extremely short: shield of mid-chest broad; sutures of the parapsides indistinct; axillæ remote from each other; scutcheon nearly elliptical: hindchest rather large, obconical, declining: petiole very short: ahdomen very much compressed, knife-shaped, longer than the chest: sheaths of the oviduct about one-sixth of the length of the abdomen: legs piceous; knees, feet, and tips of the shanks tawny: wings colourless; veins tawny; ulna shorter than the humerns; radius more than one-third of the length of the ulna; cubitus shorter than the radius; wing-brand very small. Length of the body 1 line; of the wings $1 \frac{1}{4}$ line.

Gastrancistrus Amahœus. Fem. Cyaneo-viridis, terebra abdominis dimidii longitudine, antennis nigris, pedibus flavis, alis limpidis. Page 105.

Very nearly allied to G. Dryas, but the radius is somewhat shorter, and the colour of the legs is somewhat different. Bluish green : head and chest convex, finely shagreened: head as broad as the chest: feelers black, clavate, shorter than the chest; first joint stout, linear, tawny; second cup-shaped; third and fourth very small ; fifth and following joints short, broad; club conical, about twice the length of the tenth joint: chest short, elliptical, very broad: fore-chest very short, not visible above: shield of middlechest very large; sutures of the parapsides strongly marked, approaching each other behind; scutcheon rhomboidal, rather large; axillæ separated from each other by one-third of the breadth of the shield : hind-chest short, transverse, declining: petiole very short: abdomen spindle-shaped, greenish blue, compressed, smooth, shining, concave above, keeled beneath, a little longer than the chest, but hardly more than half its breadth, rather hairy towards the tip : sheaths of the oviduct black, hairy, full half the length of the abdomen : legs yellow; tips of the feet brown: wings colourless; forewings broad; veins brown ; ulna but little shorter than the liume- 
rus; radius not half the length of the ulna; cubitus much shorter than the radius; wing-brand of moderate size. Length of the body $\frac{3}{4}$ line; of the wings $1 \frac{1}{2}$ line.

Gastrancistrus Accia. Fem. Viridis, terebra brevissima, antennis nigris, tibiis fuscis, genubus tarsisque flavis, alis limpidis. Page 105.

Allied to G. annulipes, but more slender. Bright green : head and chest convex, finely shagreened: head as broad as the chest: eyes red: feelers clavate, black, a little shorter than the chest; first joint long, slender; second cup-shaped; third aud fourth very minute; fifth and following joints transverse, short, successively increasing in breadth; club ovate-couical, broader than the tenth joint and more than twice its length: chest elliptical; fore-chest very short; shield of the middle-chest large : sutures of the parapsides strongly marked, approaching each other; scutcheon rhomboidal: hind-chest obconical, declining, of moderate size : petiole very short : abdomen slender, spindle-sbaped, smooth, shining, concave above, keeled beneath, longer and narrower than the chest; sheaths of the oviduct black, hairy, hardly one-fifth of the length of the abdomen: legs green ; shanks brown ; knees and feet yellow, tips of the latter brown: wings colourless; veins brown; ulna much shorter than the humerus; radius a little shorter than the ulna; cubitus much shorter than the radius; wing-brand small. Length of the body $\frac{3}{4}$ line; of the wings $1 \frac{1}{4}$ line.

Gastrancistrus Hamillus. Fem. AEneus, capite viridi, antennis fulvis, alis limpidis. Page 105.

Allied to G. autumnalis. Brassy: head and chest convex, finely shagreened : head green, broader than the chest: feelers subclavate, tawny, as long as the chest; first joint long, slender, yellow; second cup-shaped; third and fourth very small; fifth and following joints closely joined together, successively increasing in breadth till the tenth; club spindle-shaped, pointed, not broader than the tenth joint, but more than twice its length, narrower than that of G. autumnalis : chest brassy, nearly elliptical; fore-chest short, just visible above; shield of the middle-chest rather broad; sutures of the parapsides strongly marked; axillæ separated from each other by at least one-third of the breadth of the shield; scutcheon nearly conical ; hind-chest obconical, declining, of moderate size; petiole very short: abdomen elliptical, smooth, shining, concave above, slightly keeled beneath, narrower and a little longer 
than the chest; metapodeon long; octoon and following segments shorter: legs bright yellow; tips of feet brown: wings colourless; fore-wings broader; veins yellow; ulna about half the length of the humerus; radius shorter than the ulna ; cubitus still shorter; wingbrand small. Length of budy $\frac{3}{4}$ line; of the wings $1 \frac{1}{2}$ line.

Gastrancistrus Geganius. Mas. Purpureus, abdomine basi viridi, anlennis femoribusque fuscis, pedibus flavis, alis limpidis. Page 105.

Purple: head and chest broad, convex, finely shagreened; head a little hroader than the chest, inclining to bronze colour: eyes and eyelets or ocelli dark red: feelers 13-jointed, brown, clavate, much shorter than the chest; first joint spindle-shaped, long, yellow ; second cup-shaped, pale brown; third and fourth very small; fifth and following joints transverse, slightly cup-shaped, successively decreasing in length; club oval, broader than the tenth joint, and more than twice its length: chest oval, thick, broader in front than behind; fore-chest very short, just visible above; shield of the middle-chest broad; sutures of the parapsides rather indistinct; axillæ separated by less than one-third of the breadth of the chest; scutcheon rhomboidal ; hind-chest small, short-obconic, slightly deelining, nearly smooth, with a brassy tinge, having a slight ridge along the miridle; petiole very short : abdomen flat, linear, smooth, shining, green towards the base, hairy at the tip, a little longer than the chest, but not more than half its breadtl; metapodeon long; octoon shorter; ennaton and following segments still shorter: legs yellow; thighs and shanks rather stout, the former brown: wings colourless; veins yellow; ulna not half the length of the humerus; radius a little longer than the ulna; cubitus as long as the ulna; wing-brand very small. Length of the body 1 line; of the wings $1 \frac{1}{2}$ line.

Gastrancistrus Fidenas. Mas. Niger, antennis piceis, pedibus flavis, alis limpidis. Page 105 .

Black : head and chest black, convex, shagreened, dull; head large, broader than the chest: eyes piceous: eyelets red: feelers piceous, submoniliform, as long as the chest ; first joint yellow, long and slender; second cup-shaped; third and fourth very minute; fifth and following joints to the tenth of moderate size, suhquadrate; club ovate-conical, a little broader than the tenth joint and more than twice its length : chest nearly elliptical : fore-chest short, just visible above; shield of the middle-chest broad ; sutures of the parapsides not very distinct, approaching each other behind; scutcheon 
conical ; hind-chest large, obconical, declining : petiole short, stout : abdomen obclavate, flat, smooth, shining, a little shorter than the chest and not more than half its breadth; metapodeon long; octoon and following segments shorter: legs yellow : wings colourless ; veins pale tawny; ulna much shorter than the humerus; radius a little shorter than the ulna; cubitus much shorter than the radius; wing-brand very small. Length of the body $\frac{3}{4}$ line; of the wings $1 \frac{1}{4}$ line.

Gastrancistrus Alectus. Mas. Niger, abdomine nigro-ceneo, petiolo fulvo, pedibus flavis, alis limpidis. Page 105.

Head and chest convex, black, finely shagreened : head slightly tinged with green, broader than the chest: eyes and eyelets red: feelers brown, stout, filiform, longer than the chest; first joint yellow, long, linear; second cup-shaped; third and fourth very small; fifth and following joints to the ninth of moderate size, loosely joined together; club spindle-shaper, more than twice the length of the ninth joint; chest somewhat oval, thick, very convex, and having a slight brassy tinge; fore-chest short, just visible above; shield of the middle-chest broad; sutures of the parapsides strongly marked ; axillæ separated by one-fourth of the breadth of the chest; scutcheon large, rhomboidal, with a transverse suture before its tip; hind-chest rather large, obconical, declining, with a transverse ridge along its middle: petiole short, tawny: abdomen dark bronze, smooth, shining, depressed, oval, green towards the base, shorter and narrower than the chest; metapodeon rather long; octoon and following segments shorter; legs yellow; feet paler, their tips tawny: wings colourless; veins yellow; ulna much shorter than the humerus; radius as long as the ulna; cubitus near one half the length of the radius; wing-brand small. . Length of the body $\frac{3}{4}$ line; of the wings $1 \frac{1}{2}$ line.

Gastrancistrus Hierocles. Mas. Viridis, abdomine aneo basi subtus fulvo, antennis piceis, pedibus flavis, alis limpidis. Page 105.

Green: head and chest convex, shagreened; head a little broader than the chest; eyes red; feelers piceous, filiform, rather longer than the chest; first joint long, slender ; second cup-shaped; third and fourth very small ; fifth and following joints of nearly equal size : chest oval, rather short ; fore-chest very short; shield of the mid-chest broad; sutures of the parapsides very distinct; axillæ separated by about one-third of the breadth of the chest; scutcheon obconical; hind-chest short; petiole very short: abdomen dark 
bronze, smooth, flat, nearly linear, tawny at the base beneath, narrower and a little longer than the cbest ; legs yellow ; wings colourless; veins tawny; ulna much shorter than the humerus ; radius as long as the ulna; cubitus much shorter than the radius; wingbrand of moderate size. Length of the body $\frac{1}{3}$ line; of the wings 1 line.

Gastrancistrus Iriarte. Mas. Viridis, abdomine aneo, antennis nigris, pedibus flavis, alis limpidis. Page 105.

Bright green: head and chest convex, finely shagreened; head as broad as the chest, bright blue in front ; feelers black, filiform, rather stout, about half the length of the body; first joint green, robust; second cup-shaped; third and fourth very small; fifth and following joints of nearly equal size: chest oval; forechest very short; shield of the middle-chest rather flat; sutures of the parapsides distinct; axillæ separated by nearly one half the breadth of the chest; scutcheon nearly obconical; hind-chest rather short, obconical, slightly declining : petiole very short: abdomen smooth, flat, nearly linear, dark bronze-colour, rather shorter and narrower than the chest; metapodeon rather long; octoon and following segments of moderate size: legs yellow; hijs green: wings colourless; veins tawny; ulna much longer than the humerus ; radius not one-fourth of the length of the ulna; cubitus much shorter than the radius; wing-hrand very small. Length of the body $\frac{3}{4}$ line; of the wings $1 \frac{1}{4}$ line.

Gastrancistrus Amnisos. Mas. Aneo-viridis, abdomine purpureo basi subtus fulvo, antennis nigris, tibiis fulvis, tarsis flavis, alis subfuscis. Page 105.

Brassy green : head and chest convex, shagreened; head very little broader than the chest ; eyes dark red : feelers black, slightly moniliform, full as long as the chest ; first joint green, slender, slightly curved; sccond cup-shaped; third and fourth very small; tifth and following joints of nearly equal size : chest spindle-shaped; fore-chest short; shield of the middle-chest rather flat, much narrower in front; sutures of the parapsides indistinct; axillæ approaching very near to each other; scutcheon somewhat convex, nearly elliptical; hind-chest rather long, obconical, slightly declining: abdomen slightly increasing in breadth from the base to the tip, flat, smooth, dark purple, tawny beneath at the base, a little shorter and much narrower than the chest; metapodeon rather longer than the following segments, which are of moderate and 
nearly equal size; legs yellow; hips and thighs green; shanks tawny: wings slightly brown; veins brown; ulna much shorter than the humerus; radius a little longer than the ulna; cubitus rather long, about half the length of the radius; wing-brand small. Length of the body $1 \frac{1}{4}$ line ; of the wings $1 \frac{3}{4}$ line.

Gastrancistrus Pacilus. Fem. Purpureus viridi varius, terebra abdominis trientis longitudine, antennis nigris, femoribus aneis tibiis fuscis, tarsis flavis, alis limpidis. Page 105 .

Purple, rather hairy: head and chest convex, finely shagreened; head not broader than the chest; crown tinged with green; eyes and eyelets dark red; feelers black, clavate, rather hairy, 13-jointed, a little shorter than the chest; first joint long, linear, brassy; second cup-shaped; third and fourth very small; fifth and following joints successively decreasing in length; club conical, broader than the tenth joint and more than twice its length: chest nearly elliptical, tinged with green; fore-chest very small, hardly seen above ; shield of the middle chest rather long; sutures of rhe parapsides strongly marked ; axillæ separated by full one-third the breadth of the chest; scutcheon nearly rhomboidal; hind-chest transverse, short, slightly declining; petiole very short : abdomen smooth, shining, rather hairy, nearly elliptical, slightly varied with green, depressed above, keeled beneath, a little narrower but not shorter than the chest; metapodeon long; octoon and following segments shorter ; sheaths of the oviduct black, hairy, nearly one-third of the length of the abdomen; hips and thighs brassy; shanks brown; knees and feet yellow, tips of the latter brown: wings colourless; veins brown; ulna about half the length of the humerus; radius shorter than the ulna; cubitus shorter than the radius, very slender; wing-brand rather large. Length of the body $\frac{3}{4}$ line; of the wings $1 \frac{1}{4}$ line.

Gastrancistrus Lœlianus. Fem. Viridi-cyaneus, abdominis disco aneo, oviductu brevi, antennis nigris, tarsis flavis, alis limpidis. Page 105.

Greenish blue: head and chest convex, finely shagreened: head a little broader than the chest: eyes and eyelets red: feelers black, clavate, shorter than the chest; first joint green, long, slender; second cup-shaped; third and fourth very small; fifth and following joints short, transverse, successively decreasing in length ; club conical, broader than the tenth joint and more than twice its 
length : chest nearly oval ; fore-chest very short, hardly seen above; shield of the middle-chest broad; sutures of the parapsides strongly marked; scutcheon nearly rhomboidal; hind-chest obconic, declining, of moderate size: petiole very short: abdomen smooth, shining, bronze on the disk, depressed above, keeled beneath, decreasing in breadth from the base to the tip, narrower end much longer than the chest; sheaths of the oviduct black, hairy, nearly one-fourth of the length of the abdomen: legs green; knees yellow; feet brown : wings very slightly tinged with brown; veins brown; ulna half the length of the humerus; radius as long as the ulna; cubitus much shorter than the radius; wing-brand of moderate size. Length of the body $\frac{2}{3}$ line; of the wings 1 line.

Psilonotus Achæus. Fem. Aureo-viridis, antennis tibiisque piceis, tarsis favis, genubus et protarsis fulvis, alis limpidis. Page 105.

Head and chest bright golden green, shining, convex, finely shagreened : head as broad as the chest; front large, flat, produced into a small obtuse tooth between the feelers; the latter are inserted by the mouth, piceous, subclavate, very slender, longer than the chest; first joint long, slender, tawny, very slightly curved; second long cup-shaped; third and fourth small, but more distinct than is usual in this family; fifth and following joints subquadrate, successively decreasing in length; club spindle-shaped, a little broader than the ninth joint and more than twice its length; chest elliptical; fore-chest very short; shield of the middle-chest broad, flat; sutures of the parapsides indistinct; axillæ large, separated by nearly onethird of the breadth of the chest ; scutcheon flat, nearly rhomboidal ; hind-chest short, declining; petiole very short: abdomen lanceolate, much compressed, smooth, shining, concave above, keeled beneath, as long as the chest, but not more than one-third of its breadth : hips and thighs green; knees and fore feet tawny; shanks piceous, their tips and the four hinder feet yellow: wings colourless; veins brown; ulna full half the length of the humerus; radius shorter than the ulna; cubitus shorter than the radius; wingbrand small. Length of the body $\frac{3}{4}$ line; of the wings $1 \frac{1}{2}$ line.

\section{Macromesus. Page 106.}

Female. Head and chest convex, finely shagreened; head as broad as the chest; feelers 14 -jointed, subclavate, pubescent ; first joint long, slender; second very long, cup-shaped; third and fourth very short; fifth and following joints long, linear; club long, 
conical, more than twice the length of the eleventh joint : chest elliptical; fore-chest very short, hardly seen above; shield of the middle-chest rather long ; sutures of the parapsides strongly marked; axillæ separated by nearly one half the breadih of the chest; scutcheon long, nearly rhomboidal: hind-chest short, transverse, declining: petiole very short: abdomen spindle-shaped, smooth, shining, depressed above, hardly keeled beneath : legs long; middle shanks twice the length of the fore-shanks and a little longer than the hind-shanks; middle-feet a little longer than the other feet.

Macromesus Amphiretus. Fem. Eneus cyaneo viridi purpureoque varius, antennis piceis, pedibus fulvis, alis subfuscis. Page 106.

Body rather slender: head and chest bronze, slightly varied with green : feelers very slender, piceous, a little longer than the chest: first joint reddish yellow: front of the head varied with purple, blue and green: abdomen purple-bronze, slightly tinged with green at the base, rather hairy towards the tip, longer and a little broader than the chest: legs reddish yellow; tips of the feet brown; wings very slightly tinged with brown; veins brown; ulna rather more than half the length of the humerus; radius much shorter than the ulna; cubitus a little shorter than the radius; wingbrand of moderate size. Length of the body $1 \frac{1}{2}$ line; of the wings $2 \frac{1}{2}$ line. Allied to Merostenus and the Eupelmidæ?

Prrene Scylax. Mas. Viridis, antennis nigris, genubus, tarsis tibiisque apice fulvis, tibiis propedibusque piceis, alis limpidis. Page 106.

Dark green: head broader than the chest: eyes red: feelers black, clavate, shorter than the chest ; first joint much dilated; second cup-shaped ; third and following joints very small ; club large, oval: abdomen compressed, nearly as long as the chest: hips and thighs dark green ; knees, feet, and tips of shanks tawny; shanks and fore-feet piceous : wings colourless ; veins piceous ; ulna nearly as long as the humerus; radius not more than one-fourth of the length of the ulna; cubitus a little shorter than the radius; wingbrand small. Length of the body $\frac{3}{4}$ line; of the wings $1 \frac{1}{2}$ line.

Ormocerus Sabbas. Fem. Cyaneo-viridis, antennis fuscis basi viridibus, tibiis viridi-piceis, tarsis flavis, alis limpidis. Page 106.

Bright bluish green: head and chest convex, finely shagreened ; 
head a little broader than the chest: eyes and eyelets red: feelers brown, elavate, rather slender, a little longer than the chest; first joint green, long, slender; second cup-shaped, green; third and fourth very small; fifth and following joints successively, bit very slightly decreasing in length; club elliptical, broader than the tenth joint and more than twice its length : chest oval, broader in front than behind; fore-chest very short, just seen above; shield of the middle-chest rather long; sutures of the parapsides rather strongly marked; axillæ separated by rather less than one-fourth of the breadth of the chest; scutcheon nearly rhomboidal, of moderate size, having a transverse suture rear the tip; hind-chest short, transverse, declining; petiole very short: ahdomen spindle-shaped, smooth, shining, concave above, slightly keeled beneath, longer and much narrower' than the chest; disk slightly tinged with bronze; hips and thighs green; shanks piceous, tinged with green; knees and feet yellow, tips of the latter piceous; fore-feet brown : wings colourless; veins brown; ulna full half the length of the humerus; radius much longer than the ulna; cubitus much shorter than the ulna; wing-brand small. Length of the body $\frac{3}{4}$ line; of the wings $1 \frac{1}{2}$ line.

Ormocerus Aletes. Fem. Aureo-viridis, abdomine cyaneoviridi, antennis piceis, tibiis fulvis, pedibus favis, alis limpidis. Page 107.

Body thick and broad: head and chest golden-green, convex, finely shagreened: head very large, broarler than the chest: eyes and eyelets red: feelers 12-jointed, piceous, clavate, stout, short, not more than half the length of the chest; first joint long, linear, tawny, piceous at the tip; second cup-shaped; third and fourth very small; fifth and following joints short, transverse, successively decreasing in length; club elliptical, a little broader than the ninth joint and more than twice its length : chest deep, very robust; forechest very short; shield of the middle-chest very broad; sutures of the parapsides very indistinct; axillæ separated by one-fourth of the breadth of the chest; scutcheon truncate-conic, having a very slight trace of a transverse suture near its tip ; hind-chest of moderate size, obconical, declining, with a ridge along the middle and one on each side by which it is divided into two compartments; petiole extremely short: abdomen bluish green, smooth, shining, concave above, very deeply keeled beneath, narrower but hardly shorter than the chest; the disk is slightly tinged with bronze; metapodeon rather large; octoon and following segments of moderate size: legs green; shanks tawny; knees and tips of shanks yellow; feet yellow, their tips piceous : wings colourless; veins brown; ulna 
rather more than half the length of the humerus; radius shorter than the ulna; cubitus shorter than the radius; wing-brand small. Length of the body $1 \frac{1}{4}$ line ; of the wings 2 lines.

Cyrtogaster Pœsos. Fem. Viridis, abdomine aneo, antennis nigris, pedibus luteis, metatarsis favis, alis limpidis. Page 108.

Head and chest convex, finely shagreened : head bluish green, a little broader than the chest : eyes and eyelets red : feelers black, clavate, a little shorter than the chest ; first joint green ; second cupshaped; third and fourth very small; fifth and following joints short, and successively decreasing in length ; club elliptical, broader than the tenth joint and more than twice its length: chest green, nearly oval, not very convex; fore-chest very short; shield of the middle-chest rather short ; sutures of the parapsides very indistinct ; axillæ separated by rather more than one-fourth of the breadth of the chest : scutcheon nearly rhomboidal ; hind-chest large, obconical, declining, rather roughly shagreened : petiole moderately long: abdomen dark bronze, oval, smooth, shining, convex above, keeled beneath, a little shorter and broader than the chest; metapodeon and octoon rather large; ennaton and following segments short: legs luteous; hips green; middle-feet and hind-feet yellow, their tips piceous: wings colourless; veins tawny; ulna rather more than half the length ol the humerus; radius much shorter than the ulna; cubitus a little shorter than the radius; wing-brand small. Length of the body $\frac{2}{3}$ line; of the wings $1 \frac{1}{3}$ line.

\section{Tityros, Walker. Page 108.}

Male. Head and chest convex, finely shagreened : head very large and thick, much broader than the chest: feelers clavate, as long as the chest ; first joint nearly linear, very long and slender, nearly as long as all the following joints ; second cup-shaped ; third and fourth joints very small : fifth and following joints very short; club elliptical, much broader than the tenth joint and more than twice its length: chest short-oval, deep, broad; fore-chest very short; shield of the middle-chest large; sutures of the parapsicies indistinct; scutcheon nearly conical; hind-chest large, obconical, declining, nearly smooth: petiole short: abdomen nearly conical, depressed, smooth, shining, much shorter than the chest and not more than half its breadth; metapodeon occupying most of the back; octoon and following segments very short: ulna not more than half the length of the humerus; radius a little shorter than the ulna; cubitus much shorter than the radius; wing-brand very small. 
Trtyros Poreia. Mas. Viridis, antennis fuscis basi viridibus, tibiis tarsis que flavis, illis fulvo cinctis, protibiis et protarsis luteis, alis limpidis. Page 108.

Green : eyes and eyelets red : feelers brown; first joint green, yellow at the base; second piceous, tinged with green: hips and thighs green; tips of the latter yellow; trochanters tawny; shanks and feet of the four hinder legs yellow, the furmer traversed by a broad tawny band, tips of the latter piceous ; fore-shanks and forefeet luteous, tips of the latter brown: wings colourless; veins tawny; humerus brown. Length of the body $\frac{2}{3}$ line ; of the wings $1 \frac{1}{4}$ line.

Sphegrgaster Agriope. Mas. Cyaneo-viridis, antennis nigris, pedibus luteis, alis limpidis. Page 108.

Bright bluish green, convex : head and chest finely shagreened: head blue, much broader than the chest: eyes red: feelers black, filiform, slender, much longer than the chest; first joint yellow, slender, linear; second cup-shaped; third and fourth very small; fifth and following joints long, linear; club spindle-shaped, much longer than the preceding joint: chest nearly elliptical : fore-chest short : shield of the mid-chest much narrower in front; sutures of the parapsides distinct; axillæ large, meeting on the back; scutcheon nearly rhomboidal: hind-chest long, obconical, declining : petiole slender, cylindrical, nearly half the length of the abdomen: abdomen oval, smooth, a little narrower and very much shorter than the chest; metapodeon concave on the disk, occupying nearly half the back; octoon and ennaton of moderate size; decaton and following segments short: legs luteous; hips green: wings colourless; veins tawny; ulna very much shorter than the humerus; radius longer than than the ulna ; cubitus about half the length of the ulna; wing-brand small. Length of the body 1-1 $\frac{1}{4}$ line; of the wings $2-2 \frac{1}{4}$ lines.

Sphegigaster Lasthenes. Fem. Viridis, abdomine purpureo-aneo basi cyaneo, antennis nigris, pedibus luteis, femoribus viridibus tarsis flavis, alis limpidis. Page 108.

Dark green, convex : head and chest shagreened : head much broader than the chest: eyes dark red: feelers black, clavate, nearly as long as the chest; first joint slender, linear, luteous, black at the tip ; second cup-shaped; third and fourth very small; fifth and following joints to the tenth successively increasing in 
breadth; club conical at the tip, a little broader than the tenth joint, and more than twice its length : chest elliptical: fore-chest very short : shield of the mid-chest much narrower in front; sutures of the parapsides indistinct; axillæ large, approaching near to each other; scutcheon somewhat rhomboidal : hind-chest long, nbconical, declining, with a rim on each side : petiole stout, cylindrical, shagreened, about one-sixth of the length of the abdomen : abdomen smooth, nearly elliptical, purplish bronze on the disk, blue towards the base, deeply keeled beneath, nearly as broad as the chest but very much shorter ; metapodeon long, occupying nearly half the back; octoon of moderate size; ennaton and following segments very short: legs luteous; hips and the greater part of the thighs green; middle-feet and hind-feet jellow, their tips and those of the fore-feet piceous: wings colourless; veins piceous ; ulna little more than half the length of the humerus; radius very little longer than the una; cubitus more than half the length of the ulna; wing-brand very small. Length of the body 1 line; of the wings 2 lines.

Lamprotatos Mallius. Mas. AEneo-viridis, antennis nigris, pedibus fulvis, femoribus viridi cinctis, metatibiis apice tarsisque piceis, alis fuscis. Page 111.

Coppery green, convex : head and chest very finely shagreened, appearing almost smooth: head a little broader than the chest: eyes and eyelets red : feelers black, filiform, lunger than the chest ; first joint long, slender, green; second cup-shaped; third and fourth very small ; fifth and following joints long, linear, of nearly equal length; club spindle-shaped, much longer than the tenth joint : chest oval: fore-chest transverse, narrower in front; its length above one-fourth of its breadth; the hind border concave : shield of the mid-chest rather long; parapsides much developed, their sutures very strongly marked; axillæ rather large, parted by about one-fifth of the breadth of the chest; scutcheon subrhomboidal, with a transverse suture near its tip, a longitudinal furrow on each side, and a slight trace of a furrow proceeding from the middle of the base towards the disk : hind-chest rather large, obconical, declining; its fore-shield distinct: petiole about one-seventh of the length of the abdomen: abdomen oval, smooth, a little narrower and much shorter than the chest; metapodeon occupying nearly half of the back, its disk concave from the base till near the tip : octoon about half the length of the metapodeon; ennaton much shorter; decaton and following segments still shorter : legs tawny ; hips green; feet piceous, the joints paler towards the base; forethighs and hind-thighs traversed by a broad green band; tips 
of hind-shanks piceous: wings brown; veins piceous; humerus hardly twice the length of the ulna ; radius very much longer than the ulna; cubitus about half the length of the ulna; wing-brand large. Length of the body $1 \frac{1}{4}$ line ; of the wings $2 \frac{1}{2}$ lines.

Lamprotatus Zipoetes. Fem. Cyaneo-viridis, abdomine aneoviridi, antennis nigris, tibiis piceis, tarsis flavis, alis subfuscis. Page 111.

Convex: head and chest finely shagreened: head bluish green, much broader than the chest: eyes and eyelets red : feelers black, clavate, much shorter than the chest; first joint long, linear, green ; second cup-shaped; third and fourth very small; the following joints from the fifth to the tenth successively decreasing in length; club spindle-shaped, more than twice the length of the tenth joint : chest elliptical, greenish blue, tinged with purple; forechest transverse; its length above about one-sixth of its breadth : shield of the mid-chest long; parapsides well developed, their sutures strongly marked; axillæ parted by about one-fifth of the breadth of the chest; scutcheon subrhomboidal, with a transverse suture near its tip: hind-chest rather large, obconical, declining, with a slight furrow along the middle and a rim on each side : petiole about one-seventh of the length of the abdomen: abdomen brassy green, elliptical, smooth, keeled beneath, a little narrower and much shorter than the chest; metapodeon rather more than onethird of its length ; octoon about half the length of the metapodeon; ennaton as long as the octoon; decaton a little shorter; the following segments very short: hips and thighs green; shanks piceous; knees, feet, and tips of shanks yellow ; tips of feet brown ; foreshanks and fore-feet tawny: wings slightly tinged with brown; veins brown; ulna hardly half the length of the humerus; radius much longer than the ulna; cubitus more than half the length of the ulna ; wing-brand piceous, rather large. Length of the body 1 line; of the wings 2 lines.

Lamprotatus QEbares. Fem. Cyaneo-viridis, antennis nigris, tibiis tarsisque fulvis apice piceis, alis limpidis. Page 111.

Bluish green, convex: head and chest finely shagreened : head a little broader than the chest: eyes and eyelets red: feelers black, clavate, a little longer than the chest ; first joint green, long, slender; second cup-shaped; third and fourth very small; fifth and following joints to the tenth successively decreasing in length; 
club long-oval, broader than the tenth joint and more than twice its length : chest oval : fore-chest very short; its length above hardly one-fourth of its breadth : sutures of the parapsides strongly marked; axillæ approaching near to each other; scutcheon subrhomboidal, with a transverse suture near its tip: hind-chest large, obconical, declining: petiole short: abdomen oval, smooth, keeled beneath, a little broader and much shorter than the chest; metapodeon occupying about half of the back; octoon and following segments of moderate size: legs tawny; hips and thighs green, tips of the latter tawny; shanks and feet of four hinder legs with piceous tips: wings colourless; veins brown; ulna about half the length of the humerus; radius very much longer than the ulna; cubitus full half the length of the ulna; wing-brand small. Length of the body $\frac{2}{3}$ line; of the wings $1 \frac{1}{3}$ line.

Lamprotatus Phylander. Aneo-viridis, pedibus piceis, tarsis flavis, protarsis fulvis, alis limpidis. Page 111.

Head wanting : chest coppery green, convex, finely shagreened : fore-chest rather short; its length about one-third of its breadth; sutures of the parapsides indistinct; axillæ parted by one-fourth of the breadth of the chest; scutcheon subrhomboidal; hind-chest obconical, declining, with a slight ridge along the middle: petiole moderately long: abdomen deep, oval, smooth, convex, brassy green, slightly keeled beneath, much shorter but not narrower than the chest ; metapodeon occupying more than one-third of the back ; octoon and ennaton of moderate size; decaton and following segments very short: legs piceous; hips green; knees and feet yellow, tips of the latter brown; fore-shanks and fore-feet tawny: wings colourless; veins pale brown; ulna much shorter than the humerus; radius nearly as long as the ulna; cubitus not half the length of the radius; wing-brand very small. Length of the body $\frac{3}{4}$ line ; of the wings $1 \frac{1}{2}$ line.

Lamprotatus Acarnas. Fem. Viridis, abdominis disco purpureo, antennis nigris, pedibus flavis, alis limpidis. Page 111.

Green, convex : head and chest finely shagreened : head bluish green, a little broader than the chest: eyes and eyelets red: feelers black, clavate, a little shorter than the chest ; first joint yellow, long, linear, piceous at the tip; second long cup-shaped; third and fourth very small; fifth and the following joints to the tenth suc- 
cessively decreasing in length; club long-conical, broader than the tenth joint and more than twice its length: chest oval: fore-chest very short : shield of the mid-chest broad; sutures of the parapsides indistinct; axillæ parted by about one-fourth of the breadth of the chest; scutcheon subrhomboidal, having a slight transverse suture near its tip : hind-chest of moderate size, obconical, declining : petiole rather short: abdomen elliptical, smooth, almost flat above, slightly keeled beneath, much narrower and shorter than the chest; metapodeon occupying about half the back; octoon dark purple, large; ennaton and following segments extremely short: legs yellow; hips green; four hinder-feet pale yellow with brown tips: wings colourless; veins tawny; ulna about half the length of the humerus; radius much longer than the ulna; cubitus more than half the length of the ulna; wing-brand very small. Length of the body $\frac{2}{3}$ line; of the wings 1 line.

Lamprotatus Acilius. Mas. Viridis, antennis fuscis, pedibus flavis, alis limpidis. Page 111.

Bright green, convex, narrow: head and chest finely shagreened: head much broader than the chest: eyes and eyelets red: feelers pale brown, filiform, rather shorter than the chest ; first joint green, long, slender; second long-cup-shaped; third and fourth very small; the following joints from the fifth to the tenth successively decreasing in length; club spindle-shaped, more than twice the length of the tenth joint: chest spindle-shaped : fore-chest very short, much narrower than the middle-chest, with the hind-border nearly straight; its length above about one-fourth of its breadth: shield of the middle-chest rather long; sutures of the parapsides very distinct; axillæ parted by about one-fourth of the breadth of the chest; scutcheon subrhombiform : hind-chest rather long, obconical, declining, with a ridge along the middle and a rim on each side : petiole short: abdomen oval, smooth, hardly half the length of the chest; metapodeon occupying half of the back; octoon short; ennaton and following segments very short: legs yellow; hips green; four hinder-feet pale yellow with piceous tips: wings colourless, rather narrow; veins yellow; ulna about half the length of the humerus; radius as long as the ulna; cubitus more than half the length of the ulna; wing-brand extremely small. Length of the body $\frac{3}{4}$ line; of the wings $1 \frac{1}{3}$ line.

Lamprotatus Amulius. Fem. Viridi-aneus, antennis nigris, pedibus luteis, femoribus basi viridibus, tarsis flavis, alis limpidis. Page 111.

Greenish blue, convex : head and chest shagreened : head not 
broader than the chest: eyes and eyelets red: feelers black, subclavate, a little longer than the chest; first joint green; second cup-shaped; third and fourth very small ; fifth and following joints to the tenth successirely decreasing in length ; club spindle-shaped; much more than twice the length of the tenth joint: chest oval: fore-chest very short, narrow in front, slightly concave behind; its length above not more than one-sixth of its breadth: shield of the middle-chest broad ; sutures of the parapsides very distinct ; axillæ rather large, parted by about one-fourth of the breadth of the chest; scutcheon subrhomboidal, with a transverse suture near its tip : hind-chest large, obconical, declining: petiole very short: abdomen short-elliptical, smooth, keeled beneath, a little narrower and very much shorter than the chest; metapodeon occupying full half of the back, concave at the base; octoon and following segments short: legs luteous; hips and the lower part of the thighs green; four hinder-feet yellow with brown tips: wings colourless; veins brown; ulna about half the length of the humerus; radius very much longer than the ulna; cubitus full half the length of the ulna; wing-brand rather large. Length of the body $\frac{3}{4}$ line; of the wings $1 \frac{1}{2}$ line.

Lamprotatus Labaris. Mas. Viridis cyaneo varius, abdominis disco ceneo, antennis nigris, pedibus fulvis, tarsis flavis, profemoribus piceo vittatis, alis limpidis. Page 111.

Bright green: head and fore-chest bluish green: head and chest convex, shagreened: head a little broader than the chest: eyes and eyelets red : feelers black, filiform, longer than the chest; first joint green; second cup-shaped ; third and fourth very small; fifth and following joints successively decreasing in length; club spindle-shaped, more than twice the length of the tenth joint : chest oval : fore-chest short, narrow in front, concave behind; its breadth above more than twice its length: sutures of the parapsides very distinct; axillæ large, approaching each other; scutcheon subrhomboidal, with a transverse suture near the tip : bind-chest large, obconical, declining: petiole short: abdomen smooth, flat, nearly linear, slightly contracted at the base, narrower and very much shorter than the chest; disk bronze colour ; metapodeon large ; octoon and following segments short: legs tawny ; hips green; each fore-thigh with a piceous line along its whole length; knees and feet of four hinder-legs yellow, tips of the latter brown : wings colourless; veins piceous; humerus much longer than the ulna; radius hardly longer than the ulna; cubitus full half the length of the ulna; wing-brand very small. Length of the body $1 \frac{1}{4}$ line; of the wings $2 \frac{1}{4}$ lines. 
Lamprotatus Bolgius. Fem. Aneus, abdomine viridi vario, antennis nigris, pedibus fulvis, tarsis flavis, metafemoribus basi piceis, alis limpidis. Page 111.

Brassy, convex: head and chest shagreened : head tinged with green, hardly broader than the chest: ejes and eyelets dark red : feelers black, pubescent, subclavate, not longer than the chest; first joint long, slender, green; second cup-shaped; third and fourth very small; fifth and following joints to the tenth successively decreasing in length; club spindle-shaped, more than twice the length of the tenth joint: chest oval: fore-chest short; its breadth above more than twice its length : sutures of the parapsides distinct; axillæ rather large, parted by about one-fourth of the breadth of the chest; scutcheon nearly conical : hind-chest large, obconical, declining, with a ridge along the back: petiole short: abdomen oval, smooth, tinged with green, keeled beneath, much shorter but not narrower than the chest; metapodeon occupying about one-third of the back; octoon and three following segments of moderate size; paratelum and telum very short: legs tawny; hips brassy; base of the hind-thighs piceous ; middle-feet and hindfeet yellow with brown tips: wings colourless; veins tawny; ulna rather more than half the length of the humerus; radius much longer than the ulna; cubitus more than half the length of the ulna; wing-brand small. Length of the body $\frac{3}{4}$ lines; of the wings $1 \frac{1}{2}$ line,

Lamprotatus Methymna. Mas. et Fem. Vividis, aneo aut cyaneo varius, antennis nigris, pedibus piceis, alis fuscis. Page 111.

Male. Dark green, with a slight bluish tinge: head and chest convex, very finely shagreened, appearing almost smooth: head large, broader than the chest: feelers black, filiform, pubescent, much longer than the chest; first joint long, slender, green ; second cup-shaped; third and fourth very small ; fifth and following joints to the tenth long, linear, of equal length ; club spindleshaped, much longer than the tenth joint: chest oval, well developed : fore-chest short; its breadth above about four times its length; sutures of the parapsides very strongly developed; axillæ large, and nearly meeting on the back; scutcheon subrhomboidal, with a transverse suture near its tip : hind-chest large, obconical, declining ; its fore-shield transverse, short, distinct: petiole short: abdomen brassy green, smooth, depressed, increasing in breadth from the base to the tip, shorter and much narrower than the chest; 
metapodeon occupying much more than half of the back; octoon and ennaton of moderate size; decaton and following segments very short: legs piceous; hips green; knees and fore-shanks pale piceous: wings brown, long and large; veins dark piceous; ulna about half the length of the humerus; radius nearly twice the length of the ulna; cubitus full half the length of the ulna; wingbrand large. Length of the body 1-1 $\frac{1}{4}$ line; of the wings $2-2 \frac{1}{2}$ lines.

Var. $\boldsymbol{a}$. Head and chest green.

Var. $\beta$. Head and chest brassy green.

Female. Head broader than the chest: feelers subclavate, longer than the chest; joints from the fifth to the tenth successively decreasing in length : abdomen oval, convex above, keeled beneath, as broad as the chest, but much shorter; metapodeon occupying more than half the back; octuon rather large; ennaton and following segments very short.

Lamprotatus Pycnos. Mas. Cyaneo-viridis, abdomine cupreo vario, antennis nigris, tibiis fulvis apice tarsisque piceis, alis subgriseis. Page 111.

Bluish green : head and chest convex, shagreened: head a little broader than the chest: eyes and eyelets red: feelers black, filiform, longer than the chest; first joint green, long, slender; second cup-shaped; third and fourth very small ; fifth and following joints successively decreasing in length; club spindle-shaped, much longer than the tenth joint: chest oval: fore-chest short; its breadth above more than twice its length : sutures of the parapsides distinct; axillæ large, approaching near to each other; scutcheon subrhomboidal, with a very slight transverse suture near its tip : hind-chest blue, rather large, obconical, declining: petiole very short: abdomen smooth, flat, nearly linear, contracted at the base, varied with blue and copper-colour, shorter and rather narrower than the chest; metapodeon occupying about half of the back; octoon and ennaton of moderate size; decaton and following segments sbort: legs tawny; hips and thighs green, the latter and especially the fore-thighs tawny towards their tips ; middle-shanks and hindshanks with piceous tips, each having also a broad brown band near the base; feet piceous, hind-feet somewhat paler than the other feet: wings with a very slight gray tinge; ulna rather more than half the length of the humerus; radius much longer than the ulna; cubitus more than half the length of the ulna; wing-brand of moderate size. Length of the body $1 \frac{1}{4}$ line; of the wings 2 lines. 
Var. $\boldsymbol{a}$. Head and chest green : abdomen brassy brown: no bands across the hinder-shanks.

Female? Greenish blue: head a little broader than the chest: feelers clavate, a little longer than the chest; joints from the fifth to the tenth successively decreasing in length ; club nearly elliptical, much longer than the tenth joint: abdomen elliptical, smooth, purple, blue at the base, convex above, keeled beneath, much shorter but not narrower than the chest; metapodeon occupying one-half of the back; octoon and ennaton of moderate size; decaton and following segments very short.

Lamprotatus Coretas. Mas. Cyaneo-viridis, abdominis disco purpureo-cupreo, antennis nigris, tibiis tarsisque piceis, protibiis fulvis piceo vittatis, alis subfuscis. Page 111.

Dark green, with a slight bluish tint: head and chest convex, shagreened: head broader than the chest: eyes and eyelets dark red : feelers black, filiform, a little longer than the chest; first joint green; second cup-shaped; third and fourth very small ; fifth and following joints to the tenth successively decreasing in length; club spindle-shaped, very much longer than the tenth joint : chest oval: fore-chest short; its breadth above about twice its length: sutures of the parapsides distinct; axillæ rather large, parted by about onefourth of the breadth of the chest; scutcheon subrhomboidal, with a distinct transverse suture near its tip : hind-chest rather large, obconical, somewhat rugulose, slightly declining: petiole very short: abdomen linear, smooth, depressed, purplish copper on the disk, shorter and very much narrower than the chest; metapodeon occupying most of the back; octoon and following segments short: legs piceous; hips and thighs green; knees and fore-shanks tawny, the latter having a piceous line along their whole length: wings rather narrow, with a very slight brownish tint; veins brown; ulua about half the length of the humerus; radius much longer than the ulna; cubitus full half the length of the ulna; wing-brand piceous, rather small. Length of the body $1 \frac{1}{4}$ line; of the wings 2 lines.

Semiotus Tauriscus. Mas. Viridis, abdominis disco purpureo, antennis nigris, pedibus piceis, femoribus viridibus, protibiis et protarsis fulvis, alis subgriseis. Page 112.

Dark green: head and chest convex, finely shagreened : head not broader than the chest: eyes and eyelets dark red: feelers black, filiform, not longer than the chest; first joint long, slender, green; second cup-shaped; third and fourth very small; the fol- 
lowing joints from the fifth to the tenth successively decreasing in length; club long-conical, much longer than the tenth joint: chest elliptical : fore-chest very short; its length above not more than one-eighth of its breadth; hind-border very concave : shield of the mid-chest rather long: sutures of the parapsides distinct; axillæ parted by nearly one-third of the breadth of the chest; scutcheon subrhomboidal : hind-chest rather broad, short-obconical, slightly declining : petiole very short: abdomen oval, smooth, depressed, dark purple on the disk, a little shorter but hardly narrower than the chest; metapodeon occupying more than half of the back; octoon about one-third of the length of the metapodeon; ennaton shorter ; decaton and the following segments very short: legs tawny; hips and thighs green; four hinder-shanks and feet piceous: wings very slightly tinged with gray; veins piceous; ulna a little shorter than the humerus; radius shorter than the ulna; cubitus about half the length of the radius; wing-brand very small, forked. Length of the body 1 line; of the wings $1 \frac{3}{4}$ line.

Pteromalus Eson. Mas. Viridis, abdomine purpureo-aneo, antennis fuscis, pedibus flavis, femoribus viridibus, alis subfulvis. Page 119.

Body nearly linear : head and chest green, convex, finely shagreened: head broader than the chest: eyes and eyelets red : feelers brown, filiform, as long as the chest; first joint yellow, long, linear ; second cup-shaped; third and fourth very small ; fifth and following joints oblong-quadrate, but successively decreasing in length; club conical, more than twice the length of the tenth joint: chest nearly linear: fore-chest very short: shield of the middlechest rather long ; sutures of the parapsides indistinct; axillæ separated by rather less than one-fourth of the breadth of the chest; scutcheon subrhomboidal, having a very slight trace of a transverse suture before its tip: hind-chest of moderate size, obconical, declining : petiole very short : abdomen æneous-green, flat, smooth, shining, shorter than the chest; the disk is purplish brown; metapodeon occupying nearly one-third of the back; octoon and following segments of moderate size : legs bright yellow; hips and thighs green; tips of the feet brown: wings with a slight tawny tinge, especially beneath the ulna and the radius; ulna hardly half the length of the humerus; radius a little longer than the ulna ; cubitus shorter than the ulna; wing-brand very small. Length of the body $1 \frac{1}{4}$ line; of the wings 2 lines. 
Pteromalus Camma. Fem. Viridis, abdomine aneo, basi viridi-fulvo, antennis nigris, pedibus flavis, alis limpidis. Page 119.

Body convex : head and chest dark green, finely shagreened : head broader than the chest: eyes and eyelets red: feelers black, very slightly clavate, much longer than the chest; first joint long, slender, linear, yellow; second cup-shaped, piceous; third and fourth very small; fifth and following joints oblong, successively decreasing in length; club spindle-shaped, piceous, hardly broader than the tenth joint but more than twice its length : chest elliptical : fore-chest very short: shield of the middle-chest of moderate size; sutures of the parapsides very indistinct; axillæ separated by one-fourth of the breadth of the chest; scutcheon nearly rhomboidal : hind-chest of moderate size, obconical, declining: petiole short: abdomen elliptical, bronze, smooth, shining, greenish tawny at the base, pointed at the tip, rather deeply keeled beneath, a little narrower and longer than the chest; metapodeon rather large; octoon and following segments shorter : oviduct yellow : legs yellow; hips green; tips of feet brown: wings colourless; veins pale yellow; ulna very much shorter than the humerus; radius longer than the ulna; cubitus shorter than the ulna; wing-brand very small. Length of the body $\frac{2}{3}$ line; of the wings $1 \frac{1}{3}$ line.

Pteromalus Phasis. Mas. Cyaneo-viridis, abdominis disco purpureo-cupreo, antennis tibiisque piceis, femoribus viridibus, tarsis flavis, alis limpidis. Page 119.

Head and chest bluish green, convex, finely shagreened: head a little broader than the chest; front blue: eyes and eyelets piceous : feelers filiform, piceous, longer than the chest ; first joint long, slender, green; second cup-shaped ; third and fourth very small; the following joints from the fifth to the tenth linear, of nearly equal size ; club spindle-shaped, more than twice the length of the tenth joint: chest elliptical : fore-chest very short; its length above not more than one-tenth of its breadth; hind-border concave: shield of the mid-chest broad; sutures of the parapsides indistinct; axillæ parted by nearly one-fourth of the breadth of the chest; scutcheon subrhomboidal : hind-chest broad, declining, with a ridge along the middle and a rim on each side; petiole very short : abdomen green, purplish copper on the disk, flat, smooth, increasing in breadth from the base till near the tip, a little narrower and much shorter than the chest; metapodeon occupying nearly one-third of the back; octoon and following segments of moderate length: hips and thighs green; 
shanks piceous ; knees, feet, and tips of shanks yellow ; tips of four hinder feet brown : wings colourless; veins pale tawny; ulna about half the length of the humerus ; radius a little shorter than the ulna ; cubitus a little shorter than the radius ; wing-brand very small. Length of the body $\frac{3}{4}$ line; of the wings $1 \frac{1}{2}$ line.

Pteromalus Cyamon. Mas. Viridis, abdomine purpureo, antennis pedibusque fulvis, femoribus piceo vittatis, alis limpidis. Page 119.

Head and chest bright green, convex, finely shagreened: head a little broader than the chest: feelers dark tawny, subclavate, as long as the chest ; first joint tawny, long, linear; second cupshaped; third and fourth very small; the following joints from the fifth to the tenth successively decreasing in length; club elliptical, broader than the tenth joint and more than twice its length: chest elliptical, rather long, with a slight brassy tinge: fore-chest very short; its length above not more than one-tenth of its breadth; hind-border concave: shield of mid-chest rather long; sutures of the parapsides very indistinct; axillæ parted by about one-fourth of the breadth of the chest; scutcheon subrhomboidal : hind-chest short, declining, with a rim on each side: petiole very short: abdomen linear, flat, smooth, dark purple, a little narrower and much shorter than the chest ; metapodeon occupying more than one-third of the back; octoon rather long; the following segments short: legs pale tawny; hips green; thighs with a piceous line ahove, which line in the hind-pair extends over the whole surface of the thighs; middle-feet and hind-feet yellow with brown tips : wings colourless; veins pale yellow; ulna about half the length of the humerus; radius as long as the ulna; cubitus much shorter than the ulna; wing-brand very small. Length of the body $\frac{2}{3}$ line; of the wings $1 \frac{1}{3}$ line.

Pteromalus Hyloe. Fem. Viridis, abdomine aneo-purpureo basi viridi micante, antennis nigris, pedibus luteis, femoribus viridibus, alis subfulvis. Page 119.

Head and chest convex, finely shagreened : head dark green, a little broader than the chest: eyes and eyelets red: feelers black, clavate, rather stout, not longer than the chest; first joint long, tawny, black at the tip ; second cup-shaped; third and fourth very small; the following joints from the fifth to the tenth successively decreasing in length; club long-conical, not broader than the tenth joint but more than twice its length: chest nearly spindle- 
shaped : fore-chest very short; its length above less than one-tenth of its breadth; hind-border concave: sutures of the parapsides indistinct; scutcheon subrhomboidal, with a suture along each side: hind-chest of moderate size, obconical, declining: petiole very short: abdomen oval, smooth, coppery-purple, bright green at the base, depressed above, slightly keeled beneath, shorter but not broader than the chest; metapodeon occupying about one-fourth of its length; octoon and following segments of moderate and nearly equal size : legs bright luteous; hips and thighs green ; four hinder feet yellow with piceous tips : wings slightly tawny ; veins tawny ; ulna more than half the length of the humerus; radius longer than the ulna; cubitus much shorter than the ulna; wing-brand very small. Length of the body $1 \frac{1}{2}$ line; of the wings $2 \frac{3}{4}$ lines.

Pteromalus Acco. Fem. Aneo-viridis, abdomire purpureo aneo, basi viridi, antennis nigris, femoribus aneis, alii fulvis. Page 120.

Allied to P. deplanatus. Body dull brassy green: head and chest convex, finely shagreened: head hardly broader than the chest: eyes and eyelets red: feelers black, clavate, rather shorter than the chest; first joint tawny, very long; second long-cupshaped; third and fourth very small; the following joints from the fifth to the tenth short, closely joined together and successively decreasing in length; club conical, more than twice the length of the tenth joint : chest nearly elliptical : fore-chest very short; its length above less than one-tenth of its breadth; hind-border rather concave : shield of the mid-chest rather short and broad; sutures of the parapsides very indistinct; axillæ parted by about one-fourth of the breadth of the chest; scutcheon subrhomboidal, with a suture along each side : hind-chest of moderate size, obconical, declining, with a ridge along the middle and a rim on each side : petiole very short : abdomen nearly round, smooth, purplish bronze, green at the base, depressed above, hardly keeled beneath, much shorter than the chest, its length scarcely exceeding its breadth; metapodeon occupying hardly one-third of the back; octoon and two following segments of moderate length; the rest very short: legs tawny; hips and thighs brassy : wings tawny, rather narrow; veins dull tawny; ulna hardly half the length of the humerus; radius a little shorter than the ulna ; cubitus shorter than the radius ; wingbrand very small. Length of the body $\frac{3}{4}$ line; of the wings $1 \frac{1}{2}$ line. 
Pteromalus Tychon. Fem. Niger, abdomine aneo macula basali fulva, antennis nigris, pedibus fulvis, alis limpidis. Page 120.

Body very broad : head and chest black, convex, shagreened : head large, broader than the chest: eyes and eyelets dark red : feelers black, subclavate, much longer than the chest; first joint long, slender, tawny; second cup-shaped; third and fourth very small; the following joints from the fifth to the tenth successiyely decreasing in length ; club spindle-shaped, a little broader than the tenth joint and more than twice its length : chest short-elliptical, robust : fore-chest extremely short; its length above less than one-tenth of its breadth ; hind-border slightly concave : shield of the mid-chest broad and short; sutures of the parapsides very indistinct; axillæ parted by nearly one-fourth of the breadth of the chest; scutcheon subrhomboidal, with a slight transverse suture near its tip : hindchest large, declining, slightly obconical, with a ridge along the middle: petiole very short: abdomen short-elliptical, smooth, flat above, keeled beneath, brassy with an indistinct tawny spot near the base which is green, much shorter but hardly narrower than the chest ; its length slightly exceeding its breadth; metapodeon occupying about one-third of the back ; octoon full half the length of the metapodeon; ennaton and all the following segments short but distinct : legs tawny ; hips brassy : wings colourless; veins tawny; ulna rather less than half the length of the humerus; radius a little longer than the ulna; cubitus much shorter than the ulna; wingbrand very small. Length of the body $1 \frac{1}{4}$ line; of the wings $2 \frac{1}{2}$ lines.

Pteromalus Cergcus. Fem. Viridis, abdomine cyaneo purpureoque vario, antennis nigris, pedibus fulvo-favis, alis limpidis. Page 120.

Body bright green, rather narrow: head and chest convex, finely shagreened: head broader than the chest: eyes and eyelets red: feelers black, clavate, slender, nearly as long as the chest; first joint long, slender, yellow, slightly bent, black towards the tip ; second cup-shaped; third and fourth very small; the following joints from the tifth to the tenth successively decreasing in length ; club conical, more than twice the length of the tenth joint: chest nearly elliptical : fore-chest very short; its length above less than one-tenth of its breadth; hind-border concare : shield of the midchest rather flat; sutures of the parapsides very indistinct ; axillæ parted by nearly one-third of the breadth of the chest; scutcheon 
subrhomboidal : hind-chest rather large, obconical, declining, with a ridge along the middle and a short suture on each side: petiole very short: abdomen nearly oval, smooth, bluish green, dark coppery-purple on the disk, depressed above, hardly keeled beneath, shorter and a very little broader than the chest; metapodeon occupying less than one-fourth of the back; octoon and all the following segments of moderate and nearly equal size: legs bright tawny; hips green; shanks and feet of four hinder-legs bright yellow; tips of the feet brown: wings colourless; veins pale yellow; ulna much more than half the length of the humerus; radius a little shorter than the ulna; cubitus much shorter than the radius; wing-brand small, brown. Length of the body $1 \frac{1}{4}$ line; of the wings $2 \frac{1}{4}$ lines.

Pteromalus Arestor. Fem. Viridis, capite cyaneo-nigro, abdomine rene-viridi, antennis nigris, tibiis tarsisque flavis, alis limpidis. Page 120.

Body rather narrow : head and chest convex, finely shagreened: head bluish black, broader than the chest: eyes and eyelets dark red : feelers black, slightly clavate, nearly as long as the chest ; first joint long, slender; second cup-shaped; third and fourth very small; the following joints from the fifth to the tenth successively decreasing in length; club spindle-shaped, more than twice the length of the tenth joint: chest bright green, nearly spindle-shaped: fore-chest very short; its length not more than one-tenth of its breadth ; hind-border concave: shield of the mid-chest rather long; sutures of the parapsides very indistinct; axillæ parted by near onefourth of the breadth of the chest ; scutcheon subrhomboidal : hindchest large, obconical, slightly declining, with a ridge along the middle and a rim on each side : petiole very short: abdomen elliptical, brassy green, smooth, depressed above, hardly keeled beneath, a little broader and much shorter than the chest; metapodeon occupying nearly one-fourth of the back; octoon and the following segments of moderate size: legs bright yellow; hips and thighs green, tips of the latter yellow; tips of the feet brown: wings colourless; veins pale yellow; ulna rather more than half the length of the humerus; radius shorter than the ulna; cubitus shorter than the radius; wing-brand very small. Length of the body 1 line; of the wings 2 lines.

Pteromalus Eulimene. Fem. Viridis, abdomine cupreo-purpureo, basi viridi micante, antennis pedibusque fulvis, femoribus viridibus, alis limpidis. Page 120.

Body short, robust : head and chest dark green, couvex, finely 
shagreened: head as broad as the chest: eyes and eyelets red: feelers very dark tawny, subclavate, longer than the chest; first. joint tawny, long and slender; second cup-shaped; third and fourth very small; the following joints from the fifth to the tenth successively decreasing in length; club elliptical, broader than the tenth joint and rather more than twice its length : chest broad, very short-elliptical : fore-chest rather short; its length above about onesixth of its breadth : shield large ; sutures of the parapsides indistinct; axillæ parted by rather less than one-third of its breadth; scutcheon subrhomboidal, with a suture along each side : hind-chest transverse, very short, bright green, almost smooth, with a ridge along the middle and a rim on each side: petiole very short: abdomen oval, smooth, shining, coppery-purple, bright green at the base, depressed above, rather deeply keeled beneath, a little narrower but hardly longer than the chest; metapodeon occupying rather more than one-fourth of the back; octoon and the three following segments rather short, nearly equal ; paratelum longer ; telum very small: legs pale tawny; hips and thighs green; four hinder-feet pale yellow with brown tips : wings colourless; veins tawny; ulna less than half the length of the humerus; radius a little longer than the ulna; cubitus shorter than the ulna; wing-brand brown, small. Length of the body $\frac{3}{4}$ line; of the wings $1 \frac{1}{2}$ line.

Pteromalus Dipœnos. Fem. Aneo-viridis, abdomine aneo, antennis nigris, pedibus fulvis, femoribus piceis, alis subfulvis. Page 120.

Head and chest brassy green, convex, finely shagreened : head very little broader than the chest: eyes and eyelets piceous : feelers black, clavate, as long as the chest; first joint dark tawny, long and slender; second cup-shaped, piceous; third and fourth very small ; the following joints from the fifth to the tenth successively decreasing in length; club conical, rather broader than the tenth joint and more than twice its length: chest nearly elliptical : forechest very short; its length above less than one-tenth of its breadth: shield of the mid-chest broad and short; sutures of the parapsides very indistinct; axillæ parted by less than one-third of the breadth of the chest; scutcheon subrhomboidal, with a suture along each side: hind-chest rather short, obconical, declining: petiole very short: abdomen oval, smooth, bronze-colour, bright coppery-green at the base, depressed above, rather deeply keeled beneath, a little longer but hardly broader than the chest; metapodeon occupying about one-fourth of the back; octoon shorter than the metapodeon ; ennaton still shorter; decaton as long as the octuon; protelum 
shorter; paratelum and telum very short: legs pale tawny; hips brassy; thighs and tips of four hinder-feet piceous: wings with a slight tawny tinge; veins tawny; ulna full half the length of the humerus; radius a little shorter than the ulna; cubitus much shorter than the radius; wing-brand small. Length of the body $\frac{3}{4}$ line; of the wings $1 \frac{1}{4}$ line.

Pteromalos Glautias. Fem. Viridis, abdomine purpureo basi aureo-viridi, antennis piceis, pedibus flavis, femoribus viridibus, tibiis fusco cinctis, alis limpidis. Page 120.

Bright green: head and chest convex, finely shagreened: head a little broader than the chest: eyes and eyelets dark red: feelers dark piceous, subclavate, rather slender, hardly longer than the chest; first joint green, long, slender; second cup-shaped, third and fourth very small; the following joints from the fifth to the tenth successively decreasing in length; club nearly elliptical, rather broader than the tenth joint and more than twice its length; chest short-elliptical : fore-chest short ; its length above about oneeighth of its breadth; hinder-border slightly concave; shield of the mid-chest broad; sutures of the parapsides very indistinct; axillæ parted by one-third of the breadth of the chest; scutcheon subrhomboidal, with a suture along each side : hind-chest of moderate size, obconical, declining, with a ridge along the middle and a rim on each side: petiole very short: abdomen spindle-shaped, smooth, purple on the disk, golden green at the base, depressed above, keeled beneath, a little narrower and much longer than the chest; metapodeon occupying hardly one-fifth of the back; octoon and ennaton of nearly equal size; decaton, protelum and paratelum longer; telum short: legs yellow; hips and thighs green; a brown band round the base of each shank; four hinder-feet pale yellow with piceous tips: wings colourless; veins pale yellow; ulna less than half the length of the humerus; radius much longer than the ulna; cubitus shorter than the ulna ; wing-brand small, pale brown. Length of the body $1 \frac{1}{2}$ line; of the wings $2 \frac{1}{2}$ lines.

Preromalus Suia. Mas. Cyaneo-aut aureo-viridis, abdominis disco purpureo, antennis pedibusque fulvis, femoribus viridibus, alis limpidis. Page 120.

Head and chest bright bluish green, convex, finely shagreened : head broader than the chest: eyes and eyelets red : feelers dark tawny, clavate, rather slender, a little longer than the chest; first joint long, slender, tawny, black at the tip; second black, cup- 
shaped; third and fourth very small; the following joints to the tenth successively decreasing in length; club spindle-shaped, broader than the tenth joint and more than twice its length: chest oval: fore-chest very short ; its length less than one-tenth of its breadth : shield of the mid-chest rather long; sutures of the parapsides very indistinct; axillæ parted by one-fourth of the breadth of the chest; scutcheon subrhomboidal, with a suture along each side : hind-chest of moderate size, obconical, declining, with a ridge along the middle and a rim on each side: petiole very short; abdomen nearly linear, depressed, smooth, green, purple on the disk, a little shorter and narrower than the chest ; metapodeon occupying about one-fourth of the back; octoon and following segments short: legs tawny; hips and thighs excepting the tips of the latter green; four hinder-knees and feet pale tawny; tips of the latter brown: wings colourless; veins pale tawny; ulna less than half the length of the humerus; radius much longer than the ulna ; cubitus shorter than the radius; wing-brand small, pale brown. Length of the body 1 line; of the wings 2 lines.

Var. a. Golden green; disk of the abdomen purple: wingbrand pale tawny.

Pteromalus Amnisos. Fem. AEneus, abdomine purpureo basi viridi, antennis nigris apice piceis, pedibus flavis, femoribus viridibus, tibiis piceo cinctis, alis limpidis. Page 120.

Body brass colour : head and chest convex, finely shagreened : head as broad as the chest: eyes and eyelets dark red: feelers slender, subclavate, black, piceous at the tips, as long as the chest ; first joint long, slender, tawny at the base ; second cup-shaped; third and fourth very small; the following joints from the fifth to the tenth successively decreasing in length ; club conical at the tip, a little broader than the tenth joint and more than twice its length : chest oval: fore-chest short; its length rather more than one-tenth of its breadth: sutures of the parapsides very indistinct; axillæ parted by rather more than one-fourth of the chest ; scutcheon subrhomboidal, with a suture along each side and an indistinct transverse channel near the tip : hind-chest of moderate size, obconical, declining, with a ridge along the middle and a rim on each side: petiole very short : abdomen long-oval, smooth, purple on the disk, bright green at the base, depressed above, deeply keeled beneath, as long and nearly as broad as she chest; metapodeon occupying less than one-fourth of the back; hind-border convex; octoon and following segments of nearly equal size: legs yellow; hips and thighs green; a broad piceous band across each of the four hinder-thighs; 
fore-shanks and fore-feet tawny; four hinder-feet with piceous tips : wings colourless; veins tawny; ulna hardly half the length of the humerus; radius much longer than the ulna; cubitus slightly curved, shorter than the ulna. Length of the body $1 \frac{1}{2}$ line; of the wings $2 \frac{3}{4}$ lines.

Preromalus Zagreus. Fem. Viridis, abdomine cyaneo purpureoque vario, antennis nigris, pedibus flavis, femoribus viridibus, alis limpidis. Page 120.

Body narrow : head and chest convex, green, finely shagreened: head broader than the chest: eyes and eyelets dark red: feelers black, slender, clavate, not longer than the chest ; first joint long, slender, green, yellow at the base; second cup-shaped; third and fourth very small; the following joints from the fifth to the tenth successively decreasing in length; club nearly conical, much broader than the tenth joint, and more than twice its length: chest long, elliptical : fore-chest very short; its length full one-tenth of its breadth : shield of the mid-chest rather long ; sutures of the parapsides indistinct; axillæ parted by one-fourth of the breadth of the chest; scutcheon subrhomboidal, with a suture along each side: hind-chest of moderate size, obconical, declining, with a ridge along the middle and a rim on each side : petiole very short : abdomen spindle-shaped, smooth, dark green, tinged with blue and purple, depressed above, slightly keeled beneath, longer and a little hroader than the chest; metapodeon occupying about one-fifth of the hack; octoon and ennaton of moderate and nearly equal size; decaton, protelum, and paratelum longer; telum very small : legs bright sellow; hips and thighs green; tips of feet brown; foreshanks and fore-feet tawny : wings colourless; veins pale yellow ; ulna not half the length of the humerus; radius a little shorter than the ulna; cubitus a little shorter than the radius; wing-brand pale brown, very small. Length of the body 1 line; of the wings 2 lines.

Pteromalus Opheltes. Mas. Viridis, abdomine aneo, antennis nigris, pedibus flavis, femoribus viridibus, alis limpidis. Page 120.

Head and chest dark green, convex, finely shagreened: head broader than the chest: eyes and eyelets piceous: feelers black, subclavate, much longer than the chest ; first joint green, long, slender ; second cup-shaped; third and fourth very small; the fol. 
lowing joints to the tenth successively decreasing in length; club spindle-shaped, rather broader than the tenth joint and nearly twice its length : chest short-elliptical : fore-chest very short; its length not one-tenth of its breadth: shield of the mid-chest rather flat; sutures of the parapsides very indistinct; axillæ parted by onefourth of the breadth of the chest; scutcheon subrhomboidal, with a suture along each side: hind-chest obconical, declining, nearly smooth, with a ridge along the middle and a rim on each side : petiole very short : abdomen nearly linear, depressed, smooth, bronze colour, tinged with green at the base, a little longer and very much narrower than the chest; metapodeon occupying more than onethird of the back: legs pale yellow; hips and thighs dark green; fore-shanks and fore-feet tawny; tips of four hinder-feet brown; wings colourless; veins tawny; ulna rather thick, full baif the length of the humerus; radius nearly as long as the ulna; cubitus much shorter than the radius; wing-brand pale brown, very small. Length of the body $\frac{2}{3}$ line; of the wings $1 \frac{1}{3}$ line.

Pteromalus Mese. Mas. Aureus, abdomine purpureo, basi viridi flavoque maculato, antennis fulvis, pedibus flavis, alis limpidis. Page 120.

Body golden colour, rather narrow: head and chest convex, finely shagreened: head broader than the chest: eyes and eyelets dark red : feelers tawny, subclavate, a little shorter than the chest; first joint long, slender, bright pale yellow ; second piceous, cupshaped; third and fourth very small; the following joints to the tenth of equal breadth but successively decreasing in length; club spindle-shaped, broader than the tenth joint and more than twice its length: chest nearly spindle-shaped: fore-chest very short ; its length less than one-tenth of its breadth : parapsides very indistinct; axillæ parted by less than one-fourth of the breadth of the chest; scutcheon rather long, subrhomboidal : hind-chest well developed, obconical, declining, nearly smooth, with a ridge along the middle and a rim on each side: petiole very short: abdomen nearly linear, depressed, smooth, purple, a little narrower and much shorter than the chest, having a large yellow spot near the base which is bright green; metapodeon occupying nearly one-third of the back; octoon and following segments of nearly equal size: legs very bright pale yellow; hips gold colour: wings colourless ; veins very pale tawny; ulna full half the length of the humerus; radius as long as the ulna; cubitus much shorter than the ulna; wing.brand very small. Length of the body $\frac{2}{3}$ line; of the wings $1 \frac{1}{3}$ line. 
Pteromalus Alopius. Mas. Viridis, abdominis disco cupreo purpureo, antennis fulvis, pedibus flavis, alis limpidis. Page 120.

Very bright green : head and chest convex, finely shagreened : head much broader than the chest: eyes and eyelets piceous : feelers tawny, slender, subclavate, a little shorter than the chest; first joint long, slender, pale yellow, luteous at the tip ; second long-cupshaped; third and fourth very small; the following joints to the tenth successively decreasing in length; club spindle-shaped, a little broader than the tenth joint and more than twice its length: chest nearly elliptical : fore-chest very short; its length not more than one-tenth of its breadth : sutures of the parapsides indistinct; axillæ parted by one-fourth of the breadth of the chest ; scutcheon subrhomboidal, with a suture along each side: hind-chest golden green, short-obconical, declining, almost smocth, with a ridge along the middle and a rim on each side : petiole very short : abdomen depressed, smooth, increasing in breadth from the base till near the tip, hardly shorter or narrower than the chest; disk coppery purple; metapodeon occupying nearly one-third of the back; octoon and following segments of nearly equal size: legs bright yellow; hips green ; fore-shanks and fore-feet bright tawny ; tips of four hinderfeet brown : wings colourless ; veins pale tawny; ulna about half the length of the humerus; radius a little longer than the ulna; cubitus shorter than the ulna; wing-brand small, pale brown. Length of the body $\frac{3}{4}$ line; of the wings $1 \frac{1}{2}$ line.

Pteromalus Phylacis. Mas. Niger, tarsis flavis, alis limpidis. Page 120.

Black : head and chest convex, finely shagreened : head much broader than the chest: eyes and eyelets dark red: feelers black, slender, filiform, longer than the chest; first joint long, slender; second cup-sbaped; third and fourth very small; the following joints to the tenth successively decreasing in length; club longspindle-shaped, broader than the tenth joint and more than twice its length : chest rather long, nearly elliptical : fore-chest rather short; its length about one-sixth of its breadth: shield rather long; sutures of the parapsides indistinct; axillæ parted by one-fourth of the breadth of the chest; scutcheon subrhomboidal, with a slight impression at the base : hind-chest well developed, long, obconical, declining, with a slight ridge along the middle : petiole very short : abdomen elliptical, flat, smooth, very much shorter and narrower than the chest ; metapodeon occupying one-half of the back; octoun 
and following segments short: legs black; knees and fore-feet tawny ; four hinder-feet yellow with brown tips : wings colourless ; veins tawny; ulna more than half the length of the bumerus; radius not longer than the ulna; cubitus much shorter than the radius; wing-brand extremely small. Length of the body $\frac{3}{4}$ line ; of the wings $1 \frac{1}{2}$ line.

Pteromalus Cœno. Mas. Aureo-viridis, abdominis disco purpureo aneo, antennis nigris, pedibus fulvis, femoribus viridibus, alis limpidis. Page 120.

Golden green: head and chest convex, finely shagreened : head hardly broader than the chest: eyes and eyelets dark red: feelers black, filiform, a little longer than the chest ; first joint long, slender, yellow at the base; second cup-shaped; third and fourth very small ; the following joints to the tenth successively decreasing in length; club spindle-shaped, more than twice the length of the tenth joint: chest nearly oval: fore-chest very short; its length hardly more than one-tenth of its breadth: sutures of the parapsides rather indistinct, especially towards the hind-border of the shield; axillæ as usual with a suture along the middle, parted by full onefourth of the breadth of the chest ; scutcheon subrhomboidal, with a suture along each side, and a slight transverse channel near the tip : hind-chest rather short, slightly obconical, declining, almost smooth, with a ridge along the middle and a rim on each side: petiole very short: abdomen smooth, depressed, slightly increasing in breadth from the base till near the tip, very little shorter and narrower than the chest; disk purplish bronze; segments of nearly equal size: sexual parts brown: legs tawny; hips and thighs green; four hinder-knees and feet pale tawny; tips of the latter brown: wings colourless; veins tawny; ulna not less than half the length of the humerus; radius longer than the ulna; cubitus shorter than the ulna; wing-brand small. Length of the body $1 \frac{1}{4}$ line; of the wings $2 \frac{1}{4}$ lines.

Var. $\beta$. Abdomen bluish green.

Pteromalus Corion. Fem. Cyaneus, abdomine purpureoaneo, basi viridi micante, antennis pedibusque anticis fulvis, femoribus piceis, tibiis fuscis, tarsis flavis, alis limpidis. Page 120.

Head and chest blue, convex, finely shagreened: head a little broader than the chest: eyes and eyelets dark red: feclers tawny, slender, subclavate, a little longer than the chest; first joint pale 
tawny, long and slender; second cup-shaped; third and fourth very small ; the following joints from the fifth to the ninth successively decreasing in length: club spindle-shaped, rather broader than the tenth joint and more than twice its length: chest broad, short-elliptical : fore-chest rery short; its length less than one-tenth of its breadth : shield of the mid-chest large; sutures of the parapsides very indistinct, almost obsolete; axillæ parted by less than onethird of the breadth of the chest; scutcheon subrhomboidal, with a suture along each side : hind-chest transverse, very short, declining, bright green, almost smooth: petiole very short: abdomen oval, smooth, purplish bronze, bright green at the base, depressed above, slightly keeled beneath, much longer but not broader than the chest ; segments of moderate and nearly equal size: legs tawny; hips blue; thighs and tips of feet piceous; four hinder-legs with brown shanks and pale yellow feet: wings colourless; veins tawny; ulna less than half the length of the humerus; radius noue; cubitus much shorter than the ulna; wing-brand very small. Length of the body $\frac{3}{4}$ line; of the wings $1 \frac{1}{2}$ line.

Pteromalus Hermachus. Mas. Cupreus, abdomine basi apiceque viridi, antennis nigris, pedibus flavis, femoribus viridibus, alis limpidis. Page 120.

Copper colour: head and chest convex, finely shagreened, dark green beneath: head much broader than the chest: eyes and eyelets dark red: feelers subclavate, black, rather longer than the chest; first joint green, long, slender; second cup-shaped; third and fourth very small; the following joints to the tenth successively decreasing in length; club spindle-shaped, a little broader than the tenth joint and more than twice its length: chest longoval; fore-chest very short; its length not more than one-tenth of its breadth: sutures of the parapsides very indistinct; axillæ parted by one-fourth of the breadth of the chest; scutcheon subrhomboidal, with a suture along each side: hind-chest green, nearly smooth, obconical, declining, with a ridge along the middle and a rim on each side: petiole very short : abdomen elliptical, depressed, smooth, green towards the tip, bright green at the base, shorter and narrower than the chest; metapodeon occupying about one-third of the back: legs bright yellow; hips and thighs green ; tips of four hinder-feet pale brown : wings colourless; veins tawny; ulna about half the length of the humerus; radius very short; cubitus full twice the length of the radius, and not much shorter than the ulna; wing-brand very small. Length of the body $\frac{2}{3}$ line; of the wings $1 \frac{1}{3}$ line. 
Pteromalus Temesa. Mas. Viridis, abdomine flavo maculato, antennis luteis, pedibus flavis, alis limpidis. Page 120.

Bright green : head and chest convex, finely shagreened : head a little broader than the chest: eyes and eyelets red: feelers subclavate, luteous, longer than the chest ; first joint pale yellow, long, slender ; second cup-shaped ; third and fourth very small ; the following joints from the tenth successively decreasing in length; club spindle-shaped, a little broader than the tenth joint and nearly thrice its length: chest nearly spindle-shaped: fore-chest very short; its length above not one-tenth of its breadth: shield of the mid-chest rather flat; sutures of the parapsides very indistinct; axillæ parted by one-fourth of the breadth of the chest; scutcheon copper colour, rhomboidal : hind-chest well developed, obconical, declining, nearly smooth, with a ridge along the middle and a rim on each side: petiole very short: abdomen linear, depressed, smooth, having an indistinct yellow spot near the base, narrower and a little longer than the chest; metapodeon occupying about one-third of the back; octoon and following segments nearly equal in size: legs bright pale yellow; hips green: wings colourless; veins pale yellow; ulna hardly half the length of the humerus; radius longer than the ulna; cubitus much shorter than the ulna; wing-brand very small. Length of the body $\frac{2}{3}$ line; of the wings $1 \frac{1}{3}$ line.

Pteromalus Drepanon. Mas. Cyaneo-viridis, abdomine basi fulvo, antennis femoribusque piceis, pedibus fulvis, alis sublimpidis. Page 120.

Dark bluish green : head and chest convex, finely shagreened : head hardly broader than the chest: eyes and eyelets piceous : feelers piceous, filiform, a little longer than the chest ; first joint tawny, long, slender; second cup-shaped; third and fourth very small; the following joints to the tenth successively decreasing in length; club spindle-shaped, more than twice the length of the tenth joint: chest elliptical : fore-chest very short; its length above not more than one-tenth of its breadth : shield of the mid-chest rather flat; sutures of the parapsides very indistinct; axillæ parted by onefourth of the breadth of the chest; scutcheon subrhomboidal: hindchest of moderate size, obconical, declining, with a ridge along the middle, and a rim on each side: petiole very short: abdomen slightly increasing in breadth from the base till near the tip, depressed, smooth, tawny towards the base, nearly as long and as broad as the chest: sexual parts pale brown: legs tawny; hips 
black; thighs piceous; four hinder-knees and feet yellow: wings nearly colourless; veins tawny; ulna rather thick, much more than half the length of the humerus; radius a little longer than the ulna ; cubitus hardly half the length of the ulna; wing-brand small. Length of the body $\frac{1}{2}$ line; of the wings 1 line.

Pteromalus Belesis. Mas. Viridis, abdomine aneo basi viridi, antennis fulvis, pedibus luteis, alis limpidis. Page 121.

Head and chest bright green: head a little broader than the chest: eyes and eyelets dark red : feelers tawny, subclavate, shorter than the chest; first joint luteous, long, slender; second cupshaped; third and fourth very small; the following joints to the tenth short and successively decreasing in length; club conical, a little broader than the tenth joint, and more than twice its length : chest nearly oval : fore-chest very short; its length not one-tenth of its breadth : shield of the mid-chest short; sutures of the parapsides very indistinct; axillæ parted by one-fourth of the breadth of the chest: hind-chest transverse, very short: petiole very short: abdomen elliptical, depressed, smooth, bronze colour, bright green at the base, shorter and a little narrower than the chest; metapodeon occupying less than one-third of the back; octoon and following segments of nearly equal size: legs luteous ; hips green; four hinder-feet yellow with brown tips: wings colourless; veins dull yellow; ulna much less than half the length of the humerus; radius a little longer than the ulna; cubitus as long as the ulna; wing-brand very small. Length of the body $\frac{2}{3}$ line; of the wings $1 \frac{1}{2}$ line.

Pteromalus Lebadeia. Fem. Viridi-cupreus, antennis nigris, pedibus fulvis, alis limpidis. Page 121.

Copper colour, tinged with green: head and chest convex, finely shagreened: head much broader than the chest: eyes and eyelets dark red: feelers black, subclavate, not longer than the chest; first joint long, slender, tawny at the base; second cupshaped; third and fourth very small; the following joints to the tenth successively decreasing in length; club spindle-shaped, a little broader than the tenth joint and more than thrice its length : chest short-oval: fore-chest very short; its length not one-tenth of its breadth: shield of the mid-chest broad; sutures of the parapsides very indistinct; axillæ parted by rather more than one-fourth of the breadth of the chest; scutcheon subrhomboidal, with a suture along each side: hind-chest obconical, declining, nearly smooth, 
with a ridge along the middle and a rim on each side : petiole very short : abdomen spindle-shaped, smooth, depressed above, hardly keeled heneath, much narrower and a little longer than the chest; metapodeon occupying nearly half the back; octoon and following segments short and of nearly equal length : legs tawny ; hips green; four hinder-feet yellow with brown tips: wings colourless; veins pale tawny; ulna nearly half the length of the humerus; radius much longer than the ulna; cubitus a little shorter than the ulna; wing-brand pale brown, very small. Length of the body $\frac{3}{4}$ line ; of the wings $1 \frac{1}{2}$ line.

Pteromalus Berecynthos. Mas. Cyaneo-viridis, abdominis disco purpureo-cupreo, antennis fulvis, pedibus luteis, alis subfulvis. Page 121.

Head and chest convex, finely shagreened: head bright blue, a little broader than the chest: eyes and eyelets red : feelers tawny, subclavate, not longer than the chest; first joint long, slender, luteous; second cup-shaped ; third and fourth very small; the following joints to the tenth successively decreasing in length; club at the tip a little broader than the tenth joint and more than twice its length : chest oval, bright bluish green : fore-chest very short; its length above not one-tenth of its breadth: shield of the midchest broad; sutures of the parapsides very indistinct; axillæ parted by rather more than one-fourth of the breadth of the chest; scutcheon subrhomboidal : hind-chest short, declining : petiole very short: abdomen linear, depressed, smooth, green, hardly narrower or shorter than the chest; disk purplish copper; metapodeon occupying more than half the length of the back: legs luteous; hips green; four hinder-feet yellow with tawny tips : wings with a slight tawny tinge; veins tawny; ulna less than half the length of the humerus; radius much longer than the ulna; cubitus as long as the ulna; wing-brand very small. Length of the body 1 line; of the wings 2 lines.

Var. $\beta$. Head and chest green.

Pteromalus Lebene. Fem. Viridis, abdominis disco aneo, antennis fulvis, pedibus luteis, femoribus viridibus, alis subfulvis. Page 121.

Bright green : head and chest convex, finely shagreened : head hardly broader than the chest: eyes and eyelets red : feelers tawny, subclavate, not longer than the chest; first joint luteous, long, slender; second cup-shaped, piceous; third and fourth very small; the 
following joints to the tenth successively decreasing in length; club spindle-shaped, a little broader than the tenth joint and more than twice its length : chest nearly elliptical: fore-chest very short ; its length less than one-tenth of its breadth: shield of the mid-chest short; sutures of the parapsides very indistinct; axillæ parted by nearly one-third of the breadth of the chest ; scutcheon subrhomhoidal, with a suture along each side: hind-chest transverse, short, declining, with a ridge along the middle and a rim on each side : petiole very short: abdomen long-oval, smooth, depressed above, rather deeply keeled beneath, longer but not narrower than the chest; disk bronze colour; metapodeon occupying hardly onefourth of the chest; octoon and following segments of equal size : legs luteous; hips and thighs, except their tips,-green ; tips of four hinder-feet hrown: wings with a slight tawny tinge; veins yellow; ulna rather less than half the length of the humerus; radius a little shorter than the ulna; cubitus shorter than the radius; wing-brand very small. Length of the body 1 line; of the wings 2 lines.

Pteromalus Cercaphrus. Mas. Aneo-viridis, abdomine viridi-aneo, antennis pedibusque fulvis, femoribus viridibus, alis limpidis. Page 121.

Body short, broad: head and chest coppery-green, convex, finely shagreened: head large, broader than the chest: eyes and eyelets red : feelers tawny, clavate, a little shorter than the chest; first joint long, slender, green; second black, cup-shaped; third and fourth very small; the following joints to the tenth short and successively decreasing in length; club conical, a little broader than the tenth joint and more than twice its length: chest shortoval: fore-chest very short; its length above not more than onetenth of its breadth: shield of the mid-chest short; sutures of the parapsides very indistinct; axillæ parted by one-fifth of the breadth of the chest; scutcheon subrhomboidal, with a suture along each side and a slight transverse channel near the tip : hind-chest transverse, short, declining, nearly smooth, with a slight ridge along the middle and a rim on each side: petiole very short : abdomen conical, depressed, smooth, much narrower than the chest and hardly more than half its length, bright copper-colour tinged with green; metapodeon occupying the greater part of the back: legs tawny; hips and thighs green; tips of shanks, knees and feet of four hinderlegs yellow; tips of feet brown : wings colourless; veins yellow; ulna about half the length of the humerus; radius nearly as long as the ulna; cubitus much shorter than the radius; wing-brand very small. Length of the body $\frac{1}{2}$ line; of the wings 1 line. 
Pteromalus Lampe. Fem. Viridis, abdomine aneo-viridi disco purpureo-cupreo, antennis nigris, pedibus fulvis, alis limpidis. Page 121.

Head and chest brassy, convex, finely shagreened : head hardly broader than the chest: eyes and eyelets dark red: feelers black, clavate, a little longer than the chest; first joint long, slender, tawny; second cup-shaped; third and fourth very small; the following joints to the tenth successively decreasing in length; clnb long-conical, a little broader than the tenth joint and more than twice its length : chest elliptical : fore-chest very short ; its length above not one-tenth of its breadth : shield of the mid-chest rather short and broad; sutures of the parapsides very indistinct; axillæ parted by full one-third of the breadth of the chest; scutcheon subrhomboidal, with a suture along each side : hind-chest of moderate size, obconical, declining, almost smooth, with a ridge along the middle and a rim on each side: petiole very short: abdomen long-elliptical, smooth, brassy green, bright green at the base, depressed above, hardly keeled beneath, much longer but not narrower than the chest; disk purplish bronze; metapodeon occupying less than one-fourth of the back; octoon and four following segments of nearly equal size; telum very short: legs tawny ; hips brass colour; four hinder-feet yellow with brown tips: wings colourless; veins tawny; ulna about half the length of the humerus; radius longer than the ulna; cubitus much shorter than the ulna; wingbrand pale brown, very small. Length of the body $1 \frac{1}{4}$ line; of the wings $2 \frac{1}{4}$ lines.

Pteromalus Anchinoe. Mas. Cupreus, abdomine basi viridi micante, antennis nigris, pedibus flavis, femoribus cupreis, alis limpidis. Page 121.

Head and chest dark copper colour, convex, finely shagreened : head a little broader than the chest: eyes and eyelets dark red: feelers black, subclavate, a little longer than the chest; first joint very slender; second cup-shaped; third and fourth very small ; the following joints to the tenth successively decreasing in length; club spindle-shaped, a little broader than the tenth joint and nearly thrice its length : chest elliptical : fore-chest very short; its length not more than one-tenth of its breadth : shield of the mid-chest rather flat above; sutures of the parapsides very indistinct; axillæ parted by one-fourth of the breadth of the chest; scutcheon subrhomboidal: hind-chest smooth, declining, rather short: petiole very short : abdomen nearly linear, depressed, smooth, bright green 
at the base, shorter and narrower than the chest ; disk copper colour; metapodeon occupying less than one-third of the back ; octoon, ennaton, and decaton rather long; the following segments very short: legs yellow; hips and thighs dark copper colour; four hinder-feet with brown tips; fore-shanks and fore-feet pale tawny : wings colourless; veins tawny; ulna rather thick, more than half the length of the humerus; radius shorter than the ulna; cubitus shorter than the radius; wing-brand small, brown. Length of the body $\frac{3}{4}$ line; of the wings $1 \frac{1}{2}$ line.

Pteromalus Gallonius. Mas. Cyaneus, abdomine cupreo basi cyaneo-viridi, antennis nigris, pedibus fulvis, femoribus piceis, alis limpidis. Page 121.

Head and chest blue, convex, very finely shagreened, so as to appear almost smooth: head much broader than the chest : eyes and eyelets piceous; feelers black, subclavate, very slender, a little longer than the chest; first joint long, slender, cup-shaped; third and fourth very small; the following joints to the tenth long, linear, but successively decreasing in length ; club spindle-shaped, broader than the tenth joint and more than twice its length : chest nearly elliptical : fore-chest very short; its length above not more than onetenth of its breadth : shield of the mid-chest long, rather flat; sutures of the parapsides indistinct; axillæ parted by less than onefourth of the breadth of the chest; scutcheon subrhomboidal : hindchest rather short, obconical, declining : petiole very short : abdomen nearly linear, depressed, smooth, copper colour, bluish-green at the base, a little shorter and narrower than the chest; metapodeon occupying more than one-third of the back; octoon and following segments of nearly equal size: legs tawny; hips blue; thighs piceous ; four hinder-feet yellow with brown tips : wings colourless, rather small; veins tawny; ulna about half the length of the humerus; radius a little shorter than the ulna; cubitus nearly as long as the radius; wing-brand small. Length of the body 1 line; of the wings $1 \frac{1}{2}$ line.

Pteromalus Eleuthera. Fem. Eneo-viridis, abdomine purpureo-cupreo basi cyaneo-viridi, antennis nigris, pedibus rufis, alis subfulvis. Page 121.

Head and chest convex, shagreened: head green, a little broader than the chest: eyes and eyelets dark red : feelers black, filiform, longer than the chest ; first joint long, slender, tawny, piceous at the tip; second cup-shaped; third and fourth very small; 
the following joints to the tenth successively decreasing in length ; club long-conical, more than twice the length of the tenth joint: chest oval, coppery-green: fore-chest short; its length above less than one-tenth of its breadth; it is produced slightly in front where it is narrower, declining, and wrinkled: shield of the midchest broad; sutures of the parapsides indistinct ; axillæ parted by rather less than one-fourth of the breadth of the chest; scutcheon subrhomboidal, with a suture along each side, and a well defined transverse channel near the tip : hind-chest rather short, obconical, declining, nearly smooth, with its fore-shield distinct, and having a forked ridge along the middle and a rim on each side : petiole very short: abdumen spindle-shaped, finely shagreened, purplish copper, bluish green at the hase, depresser above, keeled beneath, hairy towards the tip, a little longer than the chest; metapodeon occupving less than one-sixth of the back; the following segments from the octoon to the paratelum successively increasing in length: legs red; hips green; feet yellow with tawny tips; sheaths of the oviduct black, hairy, appearing just beyond the tip of the abdomen : wings with a slight tawny tinge; each fore-wing with a large brown spot beneath the brand; veins piceous; ulna much less than half the length of the humerus; radius very much longer than the ulna; cubitus much shorter than the ulna; wing-brand small. Length of the body 2 lines; of the wings $3 \frac{1}{4}$ lines.

Pteromalus Lyttus. Fem. Aneo-viridis, abdomine cyaneoviridi, disco purpureo, antennis nigris, pedibus fulvis, alis limpidis. Page 121.

Head and chest brassy green, convex, finely shagreened : head a little broader than the chest: eyes and eyelets piceous: feelers black, clavate, rather hairy, much shorter than the chest; first joint long, slender, green; second cup-shaped; third and fourth very small ; the following joints to the tenth successively decreasing in length; club long-conical, rather broader than the tenth joint and more than twice its length : chest long-oval : fore-chest very short; its length hardly more than one-tenth of its brearth : shield of the mid-chest broad; sutures of the parapsides very indistinct; axillæ parted by one-fourth of the breadth of the chest; scutcheon subrhomhoidal, with a suture along each side: hind-chest of moderate size, obconical, declining, nearly smooth, with a ridge along the middle and a rim on each side : petiole very short : abdomen longelliptical, smooth, bluish-green, purple on the disk, bright green at the hase, depressed above, slightly keeled beneath, longer and a little broader than the chest; metapodeon occupying rather less than one-fourth of the back; octoon and all the following segments 
of nearly equal length: legs tawny; hips green; four hinder-feet yellow, with piceous tips: wings colourless; veins tawny; ulna a little more than half the length of the humerus; radius hardly longer than the ulna; cubitus much shorter than the ulna; wingbrand brown, small. Length of the body $1 \frac{1}{4}$ line; of the wings $2 \frac{1}{4}$ lines.

Pteromalus Bienna. Mas. Cyaneo-viridis, abdomine purpureo-cupreo, antennis piceis, femoribus viridibus, alis limpidis. Page 125.

Head and chest convex, finely shagreened: head greenish blue, a little broader than the chest: eyes arrd eyelets red : feelers piceous, nearly filiform, not longer than the chest ; first joint long, slender, tawny towards the base; second cup-shaped; third and fourth very small; fifth and following joints to the tenth rather short, nearly equal in size; club spindle-shaped, more than twice the length of the tenth joint : chest elliptical, bluish green : forechest short; its length abore little more than one-eighth of its breadth; hind-border slightly concave: sutures of the parapsides very indistinct; axillæ parted by about one-fourth of the breadth of the chest; scutcheon subrhomboidal : hind-chest of moderate size, obconical, declining: petiole very short: abdomen nearly linear, flat, smooth, purplish copper, bluish green at the base and at the tip, narrower but hardly shorter than the chest; metapodeon occupying about one-third of the back; octoon and following segments of moderate size : legs bright yellow; hips and thighs green; tips of feet piceous; fore-shanks and fore-feet tawny: wings colourless ; veins dull tawny; ulna hardly half the length of the humerus; radius much longer than the ulna; cubitus shorter than the ulna; wing-brand small. Length of the body $\frac{3}{4}$ line; of the wings $1 \frac{1}{2}$ line.

Pteromalus Patro. Mas. Cyaneo-viridis, abdomine aneo, macula viridi basali fulva, antennis fulvis, pedibus luteis, $f e$ moribus basi viridi vittatis, alis limpidis. Page 125.

Bright green with a bluish tinge: head and chest convex, finely shagreened: head broader than the chest: eyes and eyelets red: feelers dark tawny, filiform, longer thau the chest ; first joint yellow, piceous at the tip; second cup-shaped; third and fourth very small; the following joints from the fifth to the tenth successively decreasing in length; club elliptical, more than twice the 
length of the tenth joint; each joint darker towards the base : chest oval, robust: fore-chest very short; its length above about oneeighth of its breadth; hind-border slightly concave : shield of the mid-chest broad; sutures of the parapsides indistinct; axillæ parted by one-fourth of the breadth of the chest; scutcheon subrhomboidal : hind-chest of moderate size, obconical, declining, with a ridge along the middle and a rim on each side: petiole very short : abdomen bright coppery-green, smooth, flat, brouze on the disk, with a large tawny spot near the base, shorter and very much narrower than the chest; metapodeon occupying about half of the back; octoon short; ennaton and following segments still shorter: legs luteous; hips green; each thigh with a green line near its base; knees and feet of the four hinder-legs yellow; tips of the feet brown: wings colourless; veins dull tawny; ulna about half the length of the humerus; radius longer than the ulna; cubitus much shorter than the ulna; wing-brand piceous, small. Length of the body $1 \frac{1}{4}$ line; of the wings 2 lines.

Var. $\beta$. Hind-shanks yellow, with a tawny band across each.

Pteromalus Lissos. Mas. Viridis, abdominis disco aneo, antennis fulvis, pedibus flavis, alis limpidis. Page 125.

Bright green : head and chest convex, finely shagreened : head a little broader than the chest : eyes and eyelets red: feelers tawny, subclavate, not longer than the chest ; first joint yellow, long, linear; second cup-shaped; third and fourth very small; the following joints from the fifth to the tenth successively decreasing in length; club brown, conical, more than twice the length of the tenth joint: chest oval : fore-chest very short; its length less than one-tenth of its breadth; hind-border slightly concave: shield of the middlechest broad; sutures of the parapsides very indistinct; axillæ parted by one-third of the breadth of the chest ; scutcheon subrhomboidal : hind-chest short-obconical, slightly declining, with a slight ridge along the middle: petiole extremely short: abdomen nearly elliptical, smooth, flat, coppery on the disk, shorter and a little narrower than the chest; metapodeon occupying nearly one-half of the back; octoon and following segments of moderate size : legs bright yellow; hips green; tips of feet brown: wings colourless, rather large; veins pale yellow; ulna about half the length of the humerus; radius longer than the ulna; cubitus much shorter than the radius; wing-brand very small. Length of the body $1 \frac{1}{2}$ line; of the wings $3 \frac{1}{4}$ lines. 
Pteromalus Morys. Mas. Viridis, abdomine purpureo-cupreo, basi cyaneo-viridi, antennis fulvis, pedibus flavis, alis limpidis. Page 125.

Bright green: head and chest conrex, finely shagreened: head broader than the chest: eyes and eyelets red : feelers tawny, subclavate, a little longer than the chest; first joint bright yellow ; second cup-shaped; third and fourth very small; the following joints from the tifth to the tenth short and successively decreasing in leugth ; club elliptical, much more than twice the length of the tenth joint: chest nearly oval: fore-chest very short; its length above less than one-tenth of its breadth : shield of the mid-chest broad; sutures of the parapsides very indistinct; axillæ parted by nearly one-third of the breadth of the shield; scutcheon subrhomboidal: hind-chest of moderate size, short-obconical, declining, with a ridge along the middle : petiole very short : abdomen nearly linear, flat, smooth, purplish copper on the disk, bluish green at the base, a little narrower but hardly shorter than the chest; metapodeon occupying rather more than one-fourth of the back; octoon and ennaton rather large ; the following segments very short : legs bright yellow; hips green; tips of feet brown: wings colourless; veins tawny; ulna brown, very thick, more than half the length of the humerus; radius longer than the ulna; cubitus more than half the length of the ulna; wing-brand very small. Length of the body $\frac{3}{4}$ line; of the wings $1 \frac{1}{3}$ line.

Pteromalus Elyros. Mas. Viridis, abdomine purpureo-cupreo, macula basali flava, antennis nigris, pedibus flavis, metafemoribus fusco vittatis, alis limpidis. Page 125.

Body long and narrow: head and chest convex, finely shagreened: head bluish green, much broader than the chest: eyes and eyelets red : feelers black, filiform, longer than the chest; first joint long, slender, piceous, yellow towards the base; second cupshaped, piceous; third and fourth very small ; the following joints from the fifth to the tenth long, linear, but successively decreasing in length; club linear, conical at the tip, more than twice the length of the tenth joint : chest green, spindle-shaped : fore-chest very short; its length above less than one-tenth of its breadth; hind-border slightly concave : shield of the mid-chest rather long; sutures of the parapsides very indistinct; axillæ parted by ahout one-fourth of the breadth of the chest; scutcheon subrhomboidal, with a slight trace of a transverse ridge near its tip: hind-chest well developed, obconical, slightly declining, with a rim on each 
side : petiole very short: abdomen smooth, flat, linear, conical at the base and pointed at the tip, purplish copper, with a pale yellow spot near the base which is green, shorter and narrower than the chest; metapodeon occupying more than one-third of the back; octoon and following segments of moderate size: legs yellow; hips green ; a brown line along the whole length of each hind-thigh ; tips of feet brown : wings colourless; veins tawny ; ulna nearly as long as the humerus ; radius shorter than the ulna; cubitus very nearly half the length of the ulna; wing-brand very small. Length of the body $1 \frac{1}{4}$ line; of the wings 2 lines.

Pteromalus Rhytium. Mas. Viridis, abdominis disco purpureo-cupreo, antennis fuscis, pedibus flavis, alis limpidis. Page 125.

Head and chest bright green, convex, finely shagreened : head much broader than the chest: eyes and eyelets red: feelens filiform, brown, rather hairy, a little longer than the chest; first joint yellow, long, rather stout, piceous at the tip; second cup-shaped; third and fourth very small; the following joints from the fifth to the tenth successively decreasing in length; club spindle-shaped, more than twice the length of the tenth joint: chest elliptical: fore-chest very short; its length less than one-tenth of its breadth : sutures of the parapsides very indistinct; axillæ parted by onefourth of the breadith of the chest; scutcheon subrhomboidal: hind-chest rather large, obconical, declining, with a ridge along the middle : petiole very short: abdomen linear, flat, smooth, purplish copper, green at the base and at the tip, much narrower but hardly longer than the chest; metapodeon occupying rather less than onefourth of the back; octoon and following segments of moderate size: legs bright yellow; hips green; middle-feet and hind-feet pale yellow with brown tips: wings colourless; veins pale tawny; ulna rather more than half the length of the humerus; radius much shorter than the ulna; cubitus a little shorter than the ulna; wingbrand small. Length of the body 1 line; of the wings $1 \frac{3}{4}$ line.

Var. $\beta$. Chest coppery green : hind-chest bluish green.

Pteromalus Myle. Fem. Aneus, abdomine viridi disco purpureo, antennis fulvis, pedibus rufis, alis fulvis. Page 125.

Body short: head and chest convex, brassy, very finely shagreened: head large, much broader than the chest: eyes and eyelets dark red: feelers tawny, subclavate, shorter than the chest; first joint pale red, long, slender; second cup-shaped; third and 
fourth very small; the following joints from the fifth to the tenth short and successively decreasing in length ; club elliptical, pointed at the tip, longer than the tenth joint and nearly thrice its length : chest elliptical: fore-chest extremely short; its length less than one-tenth of its breadth : shield of the middle-chest short; sutures of the parapsides very indistinct; axillæ parted by one-third of the breadth of the chest; scutcheon subrhomboidal : hind-chest short : petiole very short: abdomen short-elliptical, smooth, green, depressed above, very slightly keeled beneath, much shorter but hardly narrower than the chest ; disk dark purple ; metapodeon occupying full one-third of the back ; the following segments successively decreasing in length: legs pale red; hips brassy; tips of feet piceous : wings narrow, tawny ; reins dark tawny; ulna more than half the length of the humerus; radius a little shorter than the ulna; cubitus nearly as long as the radius; wing-brand very small. Length of the body $\frac{3}{4}$ line; of the wings $1 \frac{1}{4}$ line.

Pteromalus Læta. Fem. Viridis, abdomine purpureo basi cupreo, antennis fuscis, pedibus fulvis, femoribus aneis, alis limpidis. Page 125.

Body rather narrow : head and chest green, finely shagreened : head rather short, a little broader than the chest; crown and front broad: eyes and evelets red, the former rather small: feelers brown, clavate, rather slender, much shorter than the chest; first joint tawny, slender, very long, hardly shorter than all the following joints together; second long cup-shaped; third and fourth very small; the following joints from the fifth to the tenth very short; club elliptical, pointed at the tip, and nearly thrice the length of the tenth joint: chest nearly linear, conical in front, flat above : fore-chest of moderate size, narrower in front; its breadth slightly exceeding its length : shield of the middle-chest broader than long; sutures of the parapsides very distinct on each side, but almost obsolete before they reach the hind-border; axillæ parted by onethird of the breadth of the chest ; scutcheon purple, nearly truncateconical, of moderate size, green along the hind-border : hind-chest rather large, short-obconical, nearly flat or very slightly declining, with an impression on each side of the fore-border: petiole rery short: abdomen elliptical, smooth, purple, coppery at the base, depressed above, hardly keeled beneath, a little broader but not longer than the chest: legs tawny ; hips and thighs brassy ; mid-feet and hind-feet yellow with tawny tips: wings colourless, rather narrow and short; veins pale yellow; ulna more than half the length of 
the humerus; radius less than half the length of the ulna ; cubitus longer than the radius; wing-brand very small. Length of the body 1 line; of the wings $1 \frac{1}{3}$ line.

Pteromalus Androbius. Fem. Viridis, abdomine viridi-purpureo, antennis nigris, pedibus fulvis femoribus piceis, alis limpidis. Page 126.

Allied to P. domesticus but narrower : head and chest convex, dark green, finely shagreened: head broader than the chest : eyes and eyelets dark red: feelers black, clavate, not longer than the chest; first joint tawny, long, slender; second cup-shaped; third and fourth very small; the following joints from the fifth to the tenth short and successively decreasing in length; club conical, more than twice the length of the tenth joint: chest elliptical: fore-chest very short; its length above less than one-tenth of its breadth : shield of the mid-chest short; sutures of the parapsides very indistinct; axillæ parted by almost one-fourth of the breadth of the chest; scutcheon subrhomboidal: hind-chest of moderate size, obconical, declining, with a ridge along the middle and a rim on each side : petiole very short: abdomen short-elliptical, smooth, purple, slightly tinged with green, bright green at the base, depressed above, hardly keeled beneath, a little broader but scarcely longer than the chest; metapodeon occupying about one-sixth of the back; octoon and following segments of moderate and nearly equal length: legs tawny; hips brassy green; thighs piceous; middle-feet and hind-feet pale tawny with piceous tips: wings colourless; veins tawny; ulna hardly half the length of the humerus; radius a little shorter than the ulna; cubitus shorter than the radius; wing-brand very small. Length of the body 1 line; of the wings $1 \frac{3}{4}$ line.

Pteromalus Tnatos. Fem. Cupreus, abdomine cupreo-purpureo, antennis nigris, pedibus fulvis, femoribus viridi-ceneis, alis subfulvis. Page 126.

Head and chest convex, coppery, finely shagreened: head a little broader than the chest: eyes piceous: feelers black, subclavate, shorter than the chest; first joint long, tawny till near the tip; second cup-shaped; third and fourth very small; the following joints from the fifth to the tenth successively decreasing in length ; club long-conical, more than twice the length of the tenth joint: chest short-oval: fore-chest very short; its length above not more than one-tenth of its breadth; hind-border slightly concave: su- 
tures of the parapsides very indistinct ; axillæ parted by rather less than one-fourth of the breadth of the chest; scutcheon subrhomboidal : hind-chest rather short, obconical, declining, with a ridge along the middle and a rim on each side: petiole very short: abdomen coppery-purple, smooth, nearly oval, rather slender towards the tip, depressed above, very much keeled beneath, rather broader and very much longer than the chest; a large triangular bright blnish green spot on each side of every segment; metapodeon golden green towards the base, occupying hardly more than one-eighth of the back: hind-border convex; octoon and ennaton of moderate size; decaton and the following segments rather longer : legs tawny; hips and thighs brassy green; mid-feet and hind-feet yellow with piceous tips: wings with a slight tawny tinge; veins dark tawny; ulna not more than half the length of the humerus; radius a little shorter than the ulna; cubitus much shorter than the radius; wing-brand piceous, very small. Length of the body 2 lines; of the wings 3 lines.

Preromalus Axos. Fem. Viridis, abdominis disco cupreopurpureo, antennis piceis, pedibus flavis, alis limpidis. Page 126.

Bright green: head and chest convex, finely shagreened: head broader than the chest: eyes and eyelets red : feelers clavate, dark piceous, shorter than the chest; first joint tawny, long, slender; second cup-shaped; third and fourth very small; the following joints from the fifth to the tenth hardly decreasing in length; club nearly oval, pwinted at the tip, a little broader than the tenth joint and more than twice its length: chest elliptical, with a bluish tinge: fore-chest very short; its length above not more than one-tenth of its breadth; hind-border slightly concave: sutures of the parapsides very indistinct; axillæ parted by one-fourth of the breadth of the chest ; scutcheon subrhomboidal, with a suture along each side, and a very indistinct transverse suture near the tip: hind-chest of moderate size, obconical, declining, with a ridge along the middle and a rim on each side : petiole very short: abdomen long-oval, smooth, depressed above, very slightly keeled beneath, a little broader but hardly longer than the chest; disk coppery purple, which colour spreads more along the hind-border than along the fore-border of each segment ; metapodeon not occupying more than one-sixth of the back; hind-border slightly concave; all the following segments of moderate and nearly equal length : legs yellow; hips green ; tips of feet brown; middle-feet and hind-feet pale yellow: wings colourless; veins tawny; ulna full half the 
length of the humerus; radius a little shorter than the ulna; cubitus much shorter than the radius; wing-brand small, brown. Length of the body $1 \frac{1}{4}$ line; of the wings 2 lines.

Pteromalus Otos. Fem. Viridis, abdomine cupreo-purpureo, antennis nigris, pedibus fulvis, femoribus viridibus, alis subfulvis. Page 126.

Head and chest dark green, convex, finely shagreened: head large, broader than the chest: eyes and eyelets dark red: feelers black, clavate, nearly as long as the chest ; first joint long, slender, tawny, black towards the tip; second cup-shaped; third and fourth very small; the following joints from the fifth to the tenth short and successively decreasing in length; club short, nearly conical, broader than the tenth joint and about twice its length : chest oval : fore-chest very short; its length not more than one-tenth of its breadth; sutures of the parapsides very indistinct; axillæ parted by nearly one-fourth of the breadth of the chest; scutcheon subrhomboidal, having a suture along each side, and a slight trace of a cross furrow near its tip : hind-chest rather large, obconical, slightly declining, with a ridge along the middle and a rim on each side: petiole very short: abdomen oval, smooth, coppery-purple, bright green at the hase, flat above, keeled beneath, shorter and a little broader than the chest; metapodeon occupying full one-fourth of the back; octoon and all the following segments of moderate and nearly equal size: legs tawny; hips and thighs green; tips of the latter tawny; middle-feet and hind-feet yellow with browu tips: wings with a slight tawny tinge; veins tawny; ulna about half the length of the humerus; radius a little shorter than the ulna; cubitus shorter than the radius; wing-brand very small. Length of the body $1 \frac{1}{4}$ line; of the wings 2 lines.

Pteromalus Carma. Fem. Viridis, abdominis disco purpureo, antennis fulvis, pedibus flavo-fulvis, femoribus basi piceis, alis iimpidis. Page 126.

Bright green: head and chest convex, finely shagreened: head a little broader than the chest: eyes and eyelets red: feelers dark tawny, filiform, hardly longer than the chest; first joint long, slender, yellow, piceous towards the tip ; second cup-shaped; third and fourth very small; the following joints from the fifth to the tenth equal in size and rather broader than long; club spindleshaped, thrice the length of the tenth joint: chest nearly elliptical : fore-chest very short; its length above less than one-tenth of its 
breadth ; hind-border very slightly concave : shield of the mid-chest rather short; sutures of the parapsides very indistinct; axillæ parted by about one-fourth of the breadth of the chest; scutcheon subrhomboidal : hind-chest short, slightly obconical, declining, with a ridge along the middle and a rim on each side : petiole very short: abdomen oval, smooth, depressed above, hardly keeled beneath, scarcely longer or broader than the chest; disk purple, which colour mingled with a brassy tinge extends along the hind-border of each segment; metapodeon occupying full one-sixth of the back; hind-border slightly convex; octoon and following segments of moderate and equal size : legs bright pale tawny ; hips green; thighs piceous towards the base; four hinder-knees and feet pale yellow, tips of the latter piceous : wings colourless; veins pale tawny ; ulna hardly half the length of the humerus; radius a little longer than the ulna; cubitus much shorter than the ulna; wing-brand pale brown, very small. Length of the body 1 line; of the wings 2 lines.

Pteromalus Sybritia. Fem. Viridis, cyaneo cupreo purpureoque varius, antennis nigris, pedibus favo-fulvis, femoribus piceovittatis, alis limpidis. Page 126.

Head and chest convex, finely shagreened: green slightly tinged with blue and copper colour: head large, broader than the chest : eyes and eyelets dark red : feelers black, subclavate, rather slender, piceous towards the tip ; first joint long, slender, tawny towards the base; second long-cup-shaped; third and fourth very small ; the following joints from the fifth to the tenth successively decreasing in length; club long-conical, more than twice the length lof the tenth joint: chest elliptical: fore-chest very short; its length above less than one-tenth of its breadth; hind-border slightly concave : sutures of the parapsides indistinct; axillæ parted by rather less than one-fourth of the breadth of the chest; scutcheon subrhomboidal, with a suture along each side: hind-chest rather short, declining, golden green, almost smooth, with an impression on each side of the fore-border : petiole very short: abdomen longoval, smooth, bright bluish green, depressed above, keeled beneath, very little narrower and shorter than the chest; disk purple; angle of the keel very near the base ; metapodeon occupying about onefourth of the back; octoon and three following segments of moderate size; paratelum and telum rather short: legs bright tawny; hips green; thighs with piceous stripes; four hinder-shanks and feet bright pale yellow; tips of the latter piceous : wings colourless; veins pale tawny; ulna about half the length of the humerus; ra- 
dius nearly as long as the ulna; cubitus much shorter than the radius; wing-brand very small. Length of the body $1 \frac{1}{4}$ line; of the wings $2 \frac{1}{2}$ lines.

Pteromalus Mesapos. Fem. Cupreus, abdomine cupreo-purpureo basi viridi, antennis nigris, pedibus favis, femoribus fulvis piceo vittatis, alis limpidis. Page 126.

Head and chest coppery, convex, finely shagreened: head broader than the chest: eyes and eyelets dark red: feelers black, clavate, thick, a little longer than the chest; first joint bright tawny, long, linear, black at the tip; second cup-shaped; third and fourth very small; the following joints from the tifth to the tenth successively decreasing in length; club conical, much broader than the tenth joint and more than twice its length: chest nearly oval: fore-chest very short; its length above less than one-tenth of its breadth; hind-border very concave: sutures of the parapsides very indistinct ; axillæ parted by one-fourth of the breadth of the chest ; scutcheon subrhomboidal : hind-chest rather large, obconical, declining, with some excavations along the fore-border and a slight ridge along the middle : petiole very short: abdomen oval, smooth, coppery purple, bright green at the base, depressed ahove, very deeply keeled beneath, so as almost to form a right angle, shorter and a little narrower than the chest; metapodeon occupying about one-fourth of the back, slightly excavated at the base; octoon and the following segments of moderate and nearly equal size ; paratelum and telum rather long; legs yellow, rather stout, hips brassy; thighs tawny, with piceous stripes; each middle-thigh with a spine beneath near the tip; four hinder-feet with piceous tips: wings colourless, rather short; veins pale tawny; ulna more than half the length of the humerus; radius a little shorter than the ulna; cubitus much shorter than the radius; wing-brand very small, pale brown. Length of the body $1 \frac{1}{4}$ line; of the wings 2 lines.

Pteromalus Larymna. Fem. Viridis, abdomine viridi-cyaneo, antennis nigris, pedibus fulvo-piceis, femoribus viridibus, alis limpidis. Page 126.

Bright green : head and chest convex, shagreened: head hardly as broad as the chest: eyes and eyelets bright red: feelers black, clavate, slender, much shorter than the chest; first joint long, green, slightly increasing in breadth from the base to the tip ; second long cup-shaped; third and fourth very small ; the following joints from the fifth to the tenth short and successively decreasing 
in length : club oval, much broader than the tenth joint and more than twice its length: chest elliptical: fore-chest very short; its length above about one-eighth of its breadth; hind-border very slightly concave : shield of the middle-chest rather long; sutures of the parapsides rather indistinct on each side and almost obsolete before they reach the hind-border; axillæ parted by about one-fourth of the breadth of the chest; scutcheon subrhomboidal : hind-chest short, transverse : petiole very short : abdomen oval, smooth, greenish blue, depressed above, hardly keeled beneath, a little longer and broader than the chest; metapodeon occupying about one-sixth of the back; nctoon and following segments of moderate and nearly equal length: hips and thighs green; shanks and tips of four hinder-feet piceous ; knees, feet, and tips of shanks tawny : wings colourless; veins brown; ulna less than half the length of the humerus; radius much longer than the ulna; cubitus shorter than the ulna; wing-brand small. Length of the body $1 \frac{1}{4}$ line; of the wings $2 \frac{1}{2}$ lines.

Pteromalus Etearchus. Fem. Viridis, abdomine purpureoviridi, antennis nigris, pedibus flavo-luteis, alis sulfulvis. Page 126.

Body green : head and chest convex, finely shagreened: head broader than the chest: eyes and eyelets red: feelers black, subclavate, rather slender, not longer than the chest ; first joint long, slender, tawny, black at the tip; second cup-shaped; third and fourth very small; the following joints from the fifth to the tenth successively decreasing in length; club oval, much broader than the tenth joint and more than twice its length: chest elliptical: forechest very short ; its length less than one-tenth of its breadth; hindborder slightly concave : shield of the middle-chest rather long; sutures of the parapsides very indistinct; axillæ parted by rather less than one-fourth of the breadth of the chest; scutcheon subrhomboidal, with a suture along each side: hind-chest rather short, declining, with a ridge along the middle and a rim on each side: petiole very short: abdomen spindle-shaped, smooth, depressed above, keeled and tawny beneath, narrower and very much longer than the chest; disk tinged with purple; metapodeon occupying about one-sixth of the back; octoon and three following segments of moderate and nearly equal size; paratelum and telum shorter: legs luteous; four hinder-shanks and feet bright yellow; tips of the latter brown: wings with a tawny tinge; veins tawny; ulna rather broad, about half the length of the humerus; radius a little longer than the ulna; cubitus much shorter than the ulna; wingbrand small. Length of the body $1 \frac{1}{4}$ line; of the wings $1 \frac{3}{4}$ line. 
Pteromalus Phalarsarna. Fem. Viridis, abdomine purpureocupreo, basi viridi, antennis nigris, pedibus fulvis, femoribus anticis piceo-vittatis, alis subfuscis. Page 126.

Allied to Trigonoderus. Body rather narrow : head and chest green, convex, finely shagreened: head rather large, broader than the chest: eyes and eyelets dark red: feelers black, subclavate, nearly as long as the chest; first joint green, long, slender; second cup-shaped ; third and fourth very short; the following joints, from the fifth to the tenth successively, decreasing in length; club long. conical, more than twice the length of the tenth joint: chest spindle-shaped, well developed : fore-chest rather large ; its length about one-fourth of its breadth; hind border concave: shield of the midchest narrower in front: sutures of the parapsides distinct; axillæ parted by about one-sixth of the breadth of the chest; scutcheon subrhomboidal, nearly conical in front, with a suture along each side and a transverse suture near the tip : hind-chest well developed, obconical, declining, roughly sculptured : fore-shield distinct : petiole very short: abdomen spindle-shaped, smooth, purplish copper, green at the base, depressed above, slightly keeled beneath, very little shorter and narrower than the chest; metapodeon occupying about one-fourth of the back; octoon and all the following segments of moderate and nearly equal size : legs tawny ; hips green; forethighs striped with green; four hinder feet with piceous tips: wings slightly tinged with brown; veins dull tawny; ulna a little more than half the length of the humerus; radius a little longer than the ulna: cubitus much shorter than the ulna; wing-brand very small. Length of the body $1-1 \frac{1}{3}$ line; of the wings $2-2 \frac{1}{2}$ lines. shanks.

Var. $\beta$. Thighs green; four hinder feet much paler than the

Pteromalus Themiso. Fem. Viridis, abdomine cupreo-purpureo, basi viridi micante, antennis nigris, pedibus fulvis, femoribus viridi vittatis, alis fulvis. Page 126.

Body rather narrow : head and chest dark green, convex, finely shagreened: head rather large, broader than the chest: eyes and eyelets dark red : feelers black, clavate, rather thick, nearly as long as the chest; first joint, long, linear, tawny, piceous at the tip ; second cup-shaped; third and fourth very short ; the following joints to the tenth successively decreasing in length; club conical, hardly broader than the tenth joint, but more than twice its length: chest spindle-shaped : fore-chest of moderate size ; its length about one-fourth of its breadth : shield of the mid-chest rather long : sutures of the parapsides very indistinct; axillæ parted by rather less 
than one-fourth of the breadth of the chest; scutcheon subrhomboidal : hind-chest large, obconical, declining, with a ridge along the middle and a rim on each side : petiole very short: abdomen spindle-shaped, smooth, coppery purple, bright green at the base, depressed above, keeled beneath, as long and as broad as the chest; metapodeon occupying nearly one-fourth of the back; octoon and three following segments tolerably long, nearly equal in size ; paratelum and telum short: legs tawny; hips green; thighs striped with green; four hinder-knees and feet yellow, tips of the latter tawny: wings tawny, rather narrow; veins of the same colour; ulna more than half the length of the humerus; radius a little shorter than the ulna; cubitus much shorter than the radius; wing-brand very small. Length of the body 1 line; of the wings $1 \frac{3}{4}$ line.

Pteromalus Állutius. Fem. Viridis, cyaneo cupren purpureoque varius, antennis nigris, pedibus fulvis, alis sulfulvis. Page 126.

Body rather short: head and chest convex, finely shagreened : head green, a little broader than the chest: eyes and eyelets red: feelers black, clavate, a little shorter than the chest; first joint tawur, long, and very slender; second cup-shaped; third and fourth very small; the following joints from the fifth to the tenth successively decreasing in length; club oval, much broader than the tenth joint and more than twice its length: chest elliptical, purplish copper, not much developed : fore-chest very short; its length less than one-tenth of its breadth; hind-border slightly concave: shield of the mid-chest short; sutures of the parapsides very indistinct, almost obsolete; axillæ parted by one-third of the breadth of the chest; scutcheon subrhomboidal, with a short suture along each side : petiole very short : abdomen oval, smooth, varied with blue, purple, green, and copper-colour, flat above, slightly keeled beneath, broader but scarcely longer than the chest; metapodeon occupying about one-fifth of the back; octoon and all the following segments of moderate and nearly equal length : legs tawny; hips coppery; four hinder-knees and feet yellow; tips of the feet brown: wings rather small, with a very slight tawny tinge; veins pale yellow; ulna less than half the length of the humerus; radius a little longer than the ulna; cubitus much shorter than the radius; wing-brand very small. Length of the body $\frac{3}{4}$ line; of the wings $1 \frac{1}{4}$ line. 
Pteromalus Priansos. Fem. Viridi-cyaneus, abdominis disco nigro purpureo, antennis pedibusque fulvis, femoribus cyaneoviridibus, tibiis piceo fasciatis, alis limpidis. Page 126.

Greenish blue: head and chest convex, finely shagreened: head as broad as the chest: eyes and eyelets red : feelers piceous, clavate, slender, rather shorter than the chest ; first joint long, slender, tawny at the base; second cup-shaped; third and fourth very small; the following joints from the fifth to the tenth successively decreasing in length; club oval, much broader than the tenth joint and more than twice its length: chest short-elliptical: fore-chest short; its length about one-eighth of its breadth : shield of the mid-chest rather long; sutures of the parapsides indistinct; axillæ parted by one-fourth of the breadth of the chest; scutcheon subrhomboidal, with a suture along each side: hind-chest transverse, short, declining, with a ridge along the middle and a rim on each side: petiole very short: abdomen spindle-shaped, smooth, depressed above, deeply keeled beneath, narrower and much longer than the chest; disk blackish purple; metapodeon occupying rather less than one-fourth of the back; octoon and ennaton of equal size; decaton and protelum longer; paratelum and telum shorter: legs tawny; hips and thighs bluish green; a broad piceous band across each of the four hinder-shanks; four hinder-feet pale tawny with piceous tips : wings colourless; veins pale tawny; ulna hardly half the length of the humerus; radius about as long as the ulna; cubitus much shorter than the radius: wing-brand very small. Length of the body $1 \frac{1}{4}$ line; of the wings 2 lines.

Var. $\beta$. Head and chest green.

Pteromalus Gigon. Fem. Viridis, abdomine purpureo-aneo, antennis nigris, pedibus fulvis, alis limpidis. Page 126.

Allied to P. domesticus. Body rather short: head and chest green, convex, finely shagreened: head very little broader than the chest : eyes and eyelets red : feelers black, clavate, shorter than the chest ; first joint green, long, slender ; second cup-shaped ; third and fourth very small; the following joints to the tenth short, transverse; club conical, hardly broader than the tenth joint but more than twice its length: chest elliptical, not much developed: forechest extremely short; its length above much less than one-tenth of its breadth : shield of the mid-chest short; sutures of the parapsides very indistinct, almost obsolete ; axillæ parted by full one-fourth of the chest; scutcheon subrhomboidal : hind-chest transverse, rather short, slightly declining, with a slight ridge along the middle and a 
rim on each side: petiole very short: abdomen elliptical, smooth (that is, comparatively, for in this and several other species of Pteromalus, it appears slightly shagreened when highly magnified), dark purplish bronze, brassy at the base and at the tip, depressed above, hardly keeled beneath, very little longer and broader than the chest; metapodeon as long as the octoon, hind-border slightly convex with anotch in the middle; octoon and four following segments of moderate and nearly equal size; telum very small: legs tawny; hips green; tips of the feet brown: wings colourless, rather short; veins pale tawny ; ulna rather less than half the length of the humerus; radius a little shorter than the ulna; cubitus shorter than the radius; wing-brand extremely small. Length of the body 1 line; of the wings $1 \frac{1}{2}$ line.

Pteromalus Orthagus. Fem. Cyaneo-viridis, abdomine cupreo-purpureo, antennis nigris, tibiis piceis, tarsis fulvis, alis limpidis. Page 126.

Head and chest bluish green, convex, finely shagreened : head as broad as the chest: eyes and eyelets red: feelers black, shorter and more clavate than those of P. Priansos ; first joint green, long, slender; second cup-shaped; third and fourth very small; the following joints to the tenth short, transverse; club nearly oval, broader than the tenth joint and more than twice its length: chest oval: fore-chest very short; its length not more than one-tenth of its breadth : shield of the middle-chest rather large ; sutures of the parapsides very indistinct; axillæ parted by one-fourth of the breadth of the chest; scutcheon subrhomboidal, with a suture along each side: hind-chest transverse, rather short, with a slight ridge along the middle and a rim on each side : petiole very short : abdomen spindle-shaped, smooth, blue, coppery purple on the disk, bright green at the base, depressed above, slightly keeled beneath, longer and much narrower than the chest; most of the segments of moderate and nearly equal length: hips and thighs green; shanks piceous; knees and feet tawny ; tips of four hinder-feet piceous: wings colourless; veins pale tawny; ulna hardly half the length of the humerus; radius as long as the ulna; cubitus much shorter than the radius; wing-brand very small. Length of the body $1 \frac{1}{4}$ line; of the wings 2 lines.

Pteromalus Diomedon. Fem. Purpureo-viridis, abdomine viridi-cyaneo, antennis nigris, pedibus flavo-piceis, femoribus viridibus, alis limpidis. Page 126.

Head and chest dark green tinged with purple : head very little U 2 
broader than the chest: eyes and eyelets dark red: feelers black, subclavate, rather slender, a little longer than the chest ; first joint green, long, linear ; second cup-shaped; third and fourth very small; the following joints from the fifth to the tenth successively decreasing in length; club long-oval, a little broader than the tenth joint and more than twice its length: chest short-elliptical: forechest very short ; its length above less than one-ten th of its breadth : shield of the mid-chest rather short; sutures of the parapsides indistinct; axillæ parted by little more than one-fourth of the breadth of the chest; scutcheon subrhomboidal, with a suture along each side: hind-chest golden green, short, transverse, with a slight ridge along the middle and a rim on each side: petiole very short: abdomen spindle-shaped, smooth, greenish blue, depressed above, keeled beneath, a little narrower and very much longer than the chest; metapodeon occupying nearly one-fifth of the back; octoon short; ennaton and following segments longer: hips and thighs green; shanks piceous; fore-knees and fore-feet tawny; knees, feet, and tips of shanks of four hinder-legs yellow: wings colourless; veins pale yellow; ulna about half the length of the humerus; radius as long as the ulna; cubitus much shorter than the radius; wingbrand very small. Length of the body 1 line; of the wings 2 lines.

Pteromalus Alimentus. Fem. Nigro-aneus, abdomine purpureo basi viridi, antennis femoribusque, piceis pedibus flavis, alis subfulvis. Page 126.

Head and chest convex, dull black, with a very slight brassy tinge, finely shagreened: head hardly broader than the chest: eyes and eyelets dark red : feelers dark piceous, subclavate, not longer than the chest; first joint long, slender, yellow, piceous at the tip ; second cup-shaped; third and fourth very small; the following joints from the fifth to the tentb successively decreasing in length ; club spindle-shaped, more than twice the length of the tenth joint : chest nearly oval: fore-chest very short: shield of the middlechest nearly flat; sutures of the parapsides very indistinct; axillæ parted by nearly one-third of the breadth of the chest; scutcheon subrhomboidal: hind-chest short, hardly obconical, deciining: petiole very short: abdomen spindle-shaped, smooth, dark purple, green at the base, depressed above, keeled beneath, narrower and very much longer than the chest; metapodeon occupying about onefifth of the back; octoon and following segments of moderate size and of nearly equal length : legs bright yellow; hips black; thighs except the tips, piceous; middle-feet and hind-feet pale yellow with 
black tips : wings pale tawny; a large brown spot on the disk of each fore-wing; veins pale brown; ulna hardly half the length of the humerus; radius a little longer than the ulna ; cubitus much shorter than the ulna; wing-brand very small. Length of the body $\frac{3}{4}-1$ line; of the wings $1 \frac{1}{4}-1 \frac{1}{2}$ line.

Var. $\beta$. Feelers black.

Pteromalus Cœlius. Fem. Aneus, abdomine purpureo, antennis nigris, pedibus fulvis, femoribus piceo-vittatis, proalis fusco submaculatis. Page 126.

Brassy: head and chest convex, shagreened: head a little broader than the chest : eyes and eyelets red : feelers black, almost filiform or very slightly clavate, not longer than the chest; first joint tawny, long, slender; secoud cup-shaped; third and fourth very small; the following joints from the fifth to the tenth successively decreasing in length; club spindle-shaped, more than twice the length of the tenth joint: chest oval: fore-chest extremely short: sutures of the parapsides tolerably distinct ; axillæ parted by about one-tifth of the breadth of the chest; scutcheon subrhomboidal with a transverse suture near its tip: hind-chest rather large, short-obconical, declining, with a ridge along the middle and a rim on each side; its fore-shield distinct: petiole extremely short : abdomen spindle-shaped, smooth, dark purple, depressed on the disk, hardly keeled beneath, a little narrower and much longer than the chest; metapodeon occupying about one-sixth of the back, notched in the middle of the hind-border; octoon like the following segments of moderate size, and nearly equal to the metapodeon in length : sheaths of the oviduct black, hairy : legs pale tawny ; hips brassy; a piceous line along the upper border of each of the forethighs and of the hind-thighs; tips of the feet brown: wings colourless; each fore-wing with a brown spot beneath the brand; veins tawny; ulna about half the length of the humerus; radius very much longer than the ulna; cubitus more than half the length of the ulna ; wing-brand small. Length of the body 2 lines; of the wings 3 lines.

Pteromalus Eacus. Fem. Cyaneo-viridis, abdomine purpureo nigro, antennis piceis, pedibus piceo-fulvis, alis limpidis. Page 126.

Head and chest convex, finely shagreened: head dark blue with a purple tinge, not broader than the chest: eyes and eyelets dark red: feelers piceous, clavate, not longer than the chest; first 
joint long, slender, green ; second cup-shaped; third and fourth very small ; fifth and following joints very short; club oval, much broader than the tenth joint and more than twice its length: chest oval, dark green with a blue tinge : fore-chest very short; its length above not more than one-tenth of its breadth; shield of the midchest flat; sutures of the parapsides very indistinct; axillæ parted by about one-fourth of the breadth of the chest; scutcheon subrhomboidal : hind-chest of moderate size, obconical, declining, with a suture on each side : petiole piceous, very short : abdomen nearly oval, smooth, purplish black, dark bluish green at the base, depressed above, slightly keeled beneath, a little shorter and narrower than the chest; metapodeon occupying less than half of the back; octoon and two following segments of moderate size; the other segments very short: legs pale tawny; hips dark green; thighs and shanks of four hinder-feet slightly piceous; middle-feet and hind-feet yellow with brown tips: wings colourless; veins pale tawny; ulna full half the length of the humerus; radius longer than the ulna; cubitus much shorter than the ulna; wing-brand small. Length of the body $\frac{3}{4}$ line; of the wings $1 \frac{1}{2}$ line.

Pteromalus Alope. Fem. Purpureo-niger, abdomine viridivario, antennis nigris, pedibus piceis, alis subfuscis. Page 127.

Head and chest convex, finely shagreened, black with a slight purple tinge: head not broader than the chest: eyes and eyelets piceous : feelers black : chest short-elliptical : fore-chest very short; its length above not more than one-tenth of its breadth; hind-border hardly concave: sutures of the parapsides indistinct; axillæ parted by nearly one-fifth of the breadth of the chest; scutcheon subrhomboidal : hind-chest of moderate size, nearly obconical, declining, with a ridge along the middle and a rim on each side : petiole very short: abdomen nearly elliptical, smooth, dark purple slightly tinged with green, depressed above, keeled beneath, a little broader but not longer than the chest; metapodeon nearly half the length of the back; octoon and following segments short: legs piceous; hips black: wings pale brown; veins pale brown; ulna not more than half the length of the humerus; radius longer than the ulna; cubitus more than half the length of the ulna; wing-brand small. Length of the body $\frac{3}{4}$ line; of the wings $1 \frac{1}{2}$ line. 
Preromalus Cepio. Fem. Cyaneo-viridis, abdominis disco purpureo, antennis piceis, pedibus fulvis, alis limpidis. Page 127.

Dark green : head and chest convex, finely shagreened : head dark bluish green, broader than the chest; feelers piceous; first joint tawny, slender, very long; second darker, cup-shaped; third and fourth very small ; fifth and the following joints to the tenth transverse, very short; club long-conical, more than twice the length of the tenth joint : chest nearly elliptical : fore-chest very short ; its length above about one-eighth of its breadth; hind-border concave : shield of the mid-chest rather flat; sutures of the parapsides very indistinct; axillæ parted by about one-fourth of the breadth of the chest; scutcheon subrhomboidal : hind-chest rather short, slightly obconical, declining, with a ridge on each side: petiole very short: abdomen smooth, dark green, dark purple on the disk, depressed ahove, slightly keeled beneath, a little narrower and shorter than the chest ; metapodeon uccupying about one-third of the back ; octoon and following segments of moderate size: legs pale tawny; hips dark green; thighs darker towards the base ; middle-feet and hind-feet yellow with tawny tips: wings colourless; veins pale tawny; ulna about half the length of the humerus; radius not longer than the ulna; cubitus much shorter than the radius; wingbrand very small. Length of the body $\frac{2}{3}$ line; of the wings $1 \frac{1}{4}$ line.

Pteromalus Hyrtacina. Fem. Viridis, abdominis disco cupreo-purpureo, antennis pedibusque fulvis, metafemoribus fusco vittatis, alis limpidis. Page 127.

Green: head and chest convex, finely shagreened: head broader than the chest: eyes and eyelets red: feelers tawny, subclavate, rather longer than the chest ; first joint long, slender, tawny, piceous at the tip; second cup-shaped, piceous; third and fourth very small; the following joints from the fifth to the tenth successively decreasing in length; club spindle-shaped, rather broader than the tenth joint and about thrice its length: chest nearly oval, rather short: fore-chest very short; its length less than one-tenth of its breadth: shield of the mid-chest broad; sutures of the parapsides very indistinct; axillæ parted by one-fourth of the breadth of the chest; scutcheon subrhomboidal, with a suture along each side: hind-chest transverse, rather short, with a slight ridge along the middle and a rim on each side : petiole very short : abdomen spindle-shaped, smooth, depressed above, slightly keeled 
beneath, longer and much narrower than the chest; disk coppery purple; metapodeon occupying about one-fourth of the back ; octoon and following segments of moderate length: legs pale tawny ; hips green; hind-thighs striped with brown; four hinder-knees and feet yellow, tips of the latter brown : wings colourless, ample; veins pale tawny; ulna hardly half the length of the humerus; radius a little longer than the ulna; cubitus much shorter than the ulna; wing-brand very small. Length of the body $\frac{3}{4}$ line; of the wings $1 \frac{1}{2}$ line.

Pteromalus Leuce. Fem. Aneo-viridis, abdominis disco purpureo, antennis fulvis, pedibus fulvo-flavis, alis limpidis. Page 127.

Body nearly linear, brassy green : head and chest convex, finely shagreened: head as broad as the chest: eyes and eyelets red: feelers tawny, clavate, a little longer than the chest; first joint long, linear, yellow at the base; second cup-shaped; third and fourth very small; the following joints from the fifth to the tenth successively decreasing in length; club nearly oval, much broader than the tenth joint and more than twice its length: chest shortelliptical : fore-chest very short; its length above less than onetenth of its breadth: sutures of the parapsides very indistinct; axillæ parted by one-fourth of the breadth of the chest; scutcheon subrhomboidal: hind-chest transverse, short: petiole very short: abdomen long-oval, smooth, depressed abore, keeled beneath, much longer but not narrower than the chest; disk dark purple; metapodeon and all the following segments of moderate and nearly equal size : legs very pale tawny; hips green; four hinder-shanks and feet yellow, tips of the latter tawny : wings colourless, rather narrow ; veins very pale yellow; ulna about half the length of the humerus; radius longer than the ulna; cubitus much shorter than the ulna; wing-brand very small. Length of the body $\frac{3}{4}$ line; of the wings $1 \frac{1}{4}$ line.

Pteromalus Hyrtacus. Fem. Viridi-ceneus, abdomine purpureo, antennis nigris, pedibus fulvis, femoribus piceo-vittatis, alis subfulvis. Page 127.

Head and chest convex, finely shagreened: head very dark green, a little broader than the chest: eyes and eyelets red: feelers black, slightly subclavate, a little longer than the chest; first joint tawny, long, slender, linear; second cup-shaped, piceous; third and fourth very small; the following joints from the fifth to 
the tenth successively decreasing in length; club long-conical, a little more than twice the length of the tenth joint: chest brassy; nearly elliptical: fore-chest very short ; its length above not more than one-tenth of its breadth; hind-border concave: shield of the mid-chest narrower in front; sutures of the parapsides indistinct; axillæ parted by about one-fifth of the breadth of the chest; scutchenn subrhomboidal : hind-chest rather long, obconical, declining, with a ridge along the middle and a rim on each side : petiole very short: abdomen almost spindle-shaped, depressed above, very slightly keeled beneath, smooth, coppery purple, slender towards the tip, tawny beneath and at the base above, a little longer but hardly narrower than the chest; metapodeon tinged with green, occupying about one-fourth of the back; octoon and all the following segments of moderate and nearly equal length; telum slender and pointed : legs tawny; hips brassy ; a brown streak along each of the four hinder-thighs: middle-feet and hind-feet yellow with brown tips: wings slightly tawny, rather narrow; veins brown; ulna a little more than half the length of the humerus; radius longer than the ulna; cubitus rather long, about half the length of the radius; wing-brand very small. Allied to Hetroxys. Length of the body $1 \frac{1}{4}$ line; of the wings 2 lines.

Var. $\beta$. Chest green.

Hetroxys Aponius. Mas. AEneo-viridis, abdomine purpureocupreo fulvo maculato, antennis pedibusque fulvis, femoribus piceis, alis limpidis. Fem. Abdomine immaculato, basi cyaneo-viridi, antennis nigris. Page 127.

Male. Body slender: head and chest convex, shagreened : head dark green, a little broader than the chest: eyes and eyelets red : feelers dark tawny, slender, subclavate, much longer than the chest ; first joint long, slender, tawny; second cup-shaped; third and fourth very small; the following joints to the tenth of equal breadth, but successively decreasing in length; club long-conical, a little broader than the tenth joint and nearly twice its length : chest spindle-shaped, dark coppery green : fore-chest very short; its length above not one-tenth of its breadth: shield of the mid-chest long, rather flat above; sutures of the parapsides very indistinct; axillæ parted hy one-fourth of the breadth of the chest ; scutcheon subrhomboidal, with a suture along each side, and a very indistinct transverse channel near the tip : hind-chest well developed, obconical, declining, with a ridge along the middle and a rim on each side: petiole very short: abrlomen depressed, smooth, dark purplish copper, with a tawny spot near the base, slightly increasing in 
breadth from the base till near the tip, shorter and much narrower than the chest; metapodeon not occupying more than one-sixth of the back; octoon and following segrnents of nearly equal size : legs tawny; hips green; thighs piceous towards the base; four hinder feet yellow with brown tips: wings colourless; veins tawny; ulna more than half the length of the humerus : radius much longer than the ulna; cubitus much shorter than the ulna; wing-brand large.

Female. Feelers black, a little longer than the chest ; first joint tawny: abdomen long spindle-shaped, smooth, bluish green, purplish bronze on the disk, concave above, slightly keeled beneath, much longer and narrower than the chest, hairy towards the tip; metapodeon not occupying more than one-eighth of the back: octoon and ennaton short; decaton and three following segments longer. Length of the body $1-1 \frac{1}{2}$ line; of the wings $2-2 \frac{1}{2}$ lines.

Trigonoderus Lappa. Mas. Cyaneo-viridis, abdomine purpureo-cupreo, antennis nigris, pedibus piceis, tarsis flavis, alis limpidis. Page 128.

Body narrow : head and chest convex, finely shagreened, dark hluish green: head much broader than the chest: eyes and eyelets dark red: feelers black, filiform, rather broad, shorter than the chest ; first joint long, spindle-shaped ; second cup-shaped; third and fourth very short; the following joints to the tenth successively decreasing in length; club spindle-shaped, rather more than twice the length of the tenth joint : chest long-elliptical : fore-chest long ; its length more than one-half of its breadth : shield of the mid-chest short and rather flat; sutures of the parapsides strongly marked; axillæ parted by one-fourth of the breadth of the chest; scutcheon subrhomboidal, rather flat, with a suture along each side, and a slight transverse channel near the tip : hind-chest large, obconical, slightly declining, with no ridge nor rim : petiole very short: abdomen depressed, smooth, dark purplish bronze-colour, slightly increasing in breadth from the base till near the tip, much shorter and narrower than the chest; metapodeon long: legs piceous; hips green; fore-feet pale tawny; four hinder-feet yellow with brown tips: wings colourless, rather short; veins pale tawny; ulna full half the length of the humerus; radius much longer than the ulna; cubitus a little more than half the length of the ulna; wing-brand very small. Length of the body $\frac{3}{4}-1$ line; of the wings $1 \frac{1}{4}-1 \frac{1}{2}$ line.

Var. $\beta$. Aut species distincta. Thighs and shanks dull tawny; veins of the wings dark tawny. 
Trigonoderus Tarrha. Mas. Cyaneo-viridis, abdomine purpureo-cupreo basi viridi, antennis nigris, pedibus fulvis, femoribus piceo vittatis, alis limpidis. Page 128.

Body narrow : head and chest convex, finely shagreened : head bright blue, much broader than the chest: eyes and eyelets red : feelers black, slender, hairy, very slightly subclavate, a little longer than the chest ; first joint long, slender, dark tawny ; second cupshaped; third and fourth very small; the following segments to the tenth successively decreasing in length ; club spindle-shaped, a little broader than the tenth joint, and rather more than twice its length: chest spindle-shaped, bluish green : fore-chest rather long, narrower in front; its length above more than half its breadth : shield of the mid-chest long; sutures of the parapsides strongly marked; axillæ parted by one-sixth of the breadth of the chest; scutcheon subrhomboidal, with a suture along each side, and a slight transverse channel near the tip : hind-chest large, obconical, declining, with a ridge along the middle and a rim on each side : petiole very short : abdomen depressed, smooth, purplish copper, green at the base, very slightly increasing in breadth from the base till near the tip, shorter and a little narrower than the chest; metapodeon occupying rather more than one-fourth of the back; octoon and following segments of nearly equal length: legs pale tawny; hips green; a piceous line along each thigh ; four hinder-feet yellow with brown tips : wings colourless; veins piceous; ulna much more than half the length of the humerus; radius a little longer than the ulna, reaching almost to the tip of the wing; cubitus not half the length of the ulna; wing-brand very small. Length of the body $1 \frac{1}{4}$ line; of the wings 2 lines.

Trigonoderus Alebion. Mas. Viridis, capite cyaneo, abdominis, disco purpureo-cupreo, antennis nigris, pedibus piceo-fulvis femoribus, viridibus, alis limpidis. Page 128.

Head and chest convex, shagreened : head blue, broader than the chest: eyes and eyelets red : feelers black, subclavate, a little longer than the chest; first joint long, slender, green ; second cupshaped; third and fourth very small; the following joints to the tenth of equal thickness but successively decreasing in length; club spindle-shaped, a little broader than the tenth joint and nearly twice its length : chest green, spindle-shaped : fore-chest rather long; its length more than half its breadth : shield of the mid-chest long; sutures of the parapsides rather distinct; axillæ parted by hardly onesixth of the breadth of the chest; scutcheon subrhomboidal, with a 
suture along each side, and a slight transverse channel near the tip : hind-chest large, obconical, declining, with a slight ridge along the middle and a rim on each side, and having also a transverse ridge near the tip : petiole very short: abdomen depressed, smooth, nearly linear or rather narrower at the base than at the tip, green, dark purplish copper-colour on the disk, much narrower than the chest, and hardly more than half its length; metapodeon occupying about half the back; octoon and following segments successively decreasing in length: legs tawny; hips and thighs green; four hinder legs with vellow knees and feet, and with piceous shanks and tips of feet : wings colourless; veins piceous; ulna hardly half the length of the humerus; radius much longer than the ulna; cubitus more than half the length of the ulna; wing-brand very small. Length of the body $1 \frac{1}{4}$ line; of the wings $\frac{1}{4}$ line.

Trigonoderus Polichna. Mas. Viridi-cyaneus, antennis nigris pedibus flavis, mesotibiis fulvis, alis limpidis. Page 128.

Head and chest convex, finely shagreened, very dark bluish green : head much broader than the chest: eyes and eyelets dark red: feelers black, slender, subclavate, hairy, a little shorter than the body ; first joint long, slender; second cup-shaped; third and fourth rery small; the following joints to the tenth long, but successively decreasing in leugth ; club spindle-shaped, a little broader than the tenth joint, and nearly twice its length: chest long, spindle-shaped : fore-chest narrow and declining in front; its length above about one-eighth of its breadth: shield of the mid-chest long; sutures of the parapsides indistinct; axillæ parted by rather less than one-fourth of the breadth of the chest; scutcheon subrhomboidal, with a suture along each side, and a transverse indistinct channel near the tip : hind-chest large, ohconical, declining, with no ridge nor rim: petiole very short: abdomen depressed, finely shagreened, bluish green, very slightly increasing in breadth from the base till near the tip, narrower but not shorter than the chest; metapodeon occupying about one-fifth of the back; octoon and following segments rather shorter and of equal length: legs yellow; hips green; fore-shanks and fore-feet dull yellow; middle shanks and tips of four hincier feet dark tawny: wings colourless; veins tawny; ulna rather stout, a little more than half the length of the humerus ; radius a little longer than the ulna; cubitus not half the length of the ulna; wing-brand very small. Length of the body $1 \frac{1}{4}$ line; of the wings 2 lines. 
Eupelmus Acron. Fem. Aneo-viridis, capite purpureo, antennis nigris, pedibus fulvis, femoribus tibiisque anticis piceis, alis fulvis. Page 129.

Connects Eupelmus with Trigonoderus. Head finely shagreened, nearly smooth, dark purple varied with green, clothed with white hairs in front, rather broader than the chest : eyes dark red, ratber large: feelers fourteen-jointed, black, clavate, hairy, much longer than the chest; first joint green, yellow towards the base; second cup-shaped; third and fourth very small; the following joints to the eleventh successively increasing in breadth and decreasing in length; club spindle-shaped, concave, broader than the eleventh joint and more than twice its length : chest spindle-shaped, coarsely shagreened, brassy green along the disk: fore-chest conical, abruptly narrower in front, much lower than the mid-chest; its length about half of its breadth: shield of the mid-chest nearly square, flat; sutures of the parapsides quite obsolete; axillæ parted by rather less than one-fourth of the breadth of the chest; scutcheon subrhomboidal, with a suture along each side: hind-chest and petiole very short: abdomen lance-shaped, brassy green, concave on the disk, slightly keeled beneath, hairy towards the tip, about twice the length and half the breadth of the chest; metapodeon occupying about one-sixth of the back; the following segments of nearly equal length: leg's tawuy; hips green; tips of feet brown : forelegs short and stout with piceous thighs and shanks; middle-legs dilated as usual: wings tawny; veins also tawny; ulna much more than half the length of the humerus; radius a little shorter than the ulna; cubitus about half the length of the ulna, slightly curved; wing-brand very snall. Length of the body 2 lines; of the wings 3 liues.

\section{Metallon (n. g.)}

Body narrow: head a little broader than the chest: feelers 10-jointed ; first joint long, slender; second cup-shaped ; third and following joints broader and successively decreasing in length: forechest of moderate size, short-conical, slightly wrinkled across the fore-part; its length about one-half of its breadth: shield of the mid-chest short; axillæ parted by a very short interval on the back; scutcheon subrhomboidal : hind-chest of moderate size, obconical, slightly declining: petiole very short: abdomen convex, smooth, elliptical ; metapodeon short, hind-border slightly convex; octoon much longer; ennaton and decaton much shorter; paratelum and telum short. 
Metallon Acacallis. Fem. Fulvus, vividi varius, abdomine cupreo, antennis nigris apice fulvis, pedibus fulvis Page 129.

Body tawny: eyes and eyelets dark red: head smooth, shining; crown tinged with green : feelers black, clavate, hairy, tawny at the tips and towards the base, a little shorter than the chest; the four basal joints, the fifth joint beneath, and the tenth joint are tawny: chest convex, smooth, shining, with a few punetures ; axillæ green; scutcheon with a green line along the middle: abdomen shorter but hardly broader than the chest, copper-colour, tawny towards the base which is bright green : legs tawny ; hind-feet pale yellow with piceous tips: wings rudimentary. Length of the body $\frac{3}{4}$ line.

Encyrtus Alycœus. Fem. Viridis, abdomine nigro-aneo, antennis femoribusque nigris, tibiis piceis. tarsis flavis, alis subgriseis. Page 135.

Body short, broad : head and chest convex, dark green, punctured, rather hairy: head as broad as the chest: eyes dark red: feeler's black, clavate, not longer than the chest; first joint long, slender, slightly spindle-shaped : abdomen depressed above, smooth, shining, bronze-black, dark green at the base, much shorter but not narrower than the chest; its length not exceeding its breadth: legs black; shanks piceous; knees and feet dull yellow; tips of the latter piceous: wings slightly tinged with gray; veins piceous. Length of the body $\frac{1}{3}$ line; of the wings $\frac{2}{3}$ line.

Encyrtus Molos. Mas. Cyaneo-viridis, abdomine aneo, antennis pedibusque nigris, tarsis flavis, alis limpidis. Page 135.

Head and chest bluish green, convex, shagreened: head as broad as the chest: eyes dark red: feelers black, nearly filiform, rather broad, much longer than the chest ; first joint long, slender ; second cup-shaped; the following joints nearly equal in length; club spindle-shaped, full twice the length of the eighth joint : shield of the middle-chest long; scutcheon also long, nearly obconical : abdomen dark bronze colour, smooth, shining, depressed above, narrower than the chest and hardly more than half its length: legs black; knees and feet yellow ; tips of the latter piceous; fore-feet tawny: wings colourless; veins piceous. Length of the body $\frac{1}{2}-\frac{3}{4}$ line; of the wings $1-1 \frac{1}{2}$ line. 
Encyrtus Phrosime. Fem. Ater, capite viridi, oviductu abdominis dimidii longitudine, tarsis flavis, protibiis et protarsis fulvis, alis limpidis. Page 135.

Head and chest convex: head green, as broad as the chest; crown narrow : eyes piceous, rather large: feelers black, clavate, not longer than the chest: chest broad, of a velvet-like black colour: abdomen black, much compressed, pointed, not more than one-half of the length of the breadth of the chest: sheaths of the oviduct projecting beyond the tip of the abdomen for about half its length : legs black; fore-shanks and fore-feet tawny ; four hinderfeet pale yellow with brown tips : wings colourless; humerus tawny; cubitus yellow, forming a very acute angle with the radius, which like the ulna is almost black. Length of the body $\frac{1}{2}$ line; of the wings 1 line.

Encyrtus Tylissos. Mas. Fuscus, pedibus fulvis, alis limpidis perangustis. Page 135 .

Body brown : head very short, as broad as the chest, which is rather flat: eyes piceous: feelers brown, hairy, filiform, nearly as long as the body ; first joint stout, almost spindle-shaped : abdomen linear, flat, a little paler and longer but not narrower than the chest: legs tawny: wings colourless, very narrow; veins tawny. Length of the body $\frac{1}{3}$ line; of the wings $\frac{2}{3}$ line.

Encyrtus Iophon. Mas. Niger, tarsis fulvis, alis limpidis. Page 135.

Body black, stout: head a little narrower than the chest : eyes piceous : feelers black, subclavate, longer than the chest; first joint long, slender; second cup-shaped; the following joints from the fourth successively decreasing in length: chest broad, coarsely punctured: abdomen nearly linear, smooth, much shorter and narrower than the chest: legs black; knees and feet tawny: wings colourless; veins tawny. Length of the body $\frac{1}{2}$ line; of the wings 1 line.

Encyrtus Acratos. Niger, abdomine nigro-aneo, pedibus piceis, alis limpidis. Page 135.

Body black, short, broad : head nearly as broad as the chest which is slightly convex : eyes piceous, rather large : feelers black, 
slender, subclavate, not longer than the chest ; first joint long, slender; second cup-shaped; the following joints successively decreasing in length ; club spindle-shaped, a little broader than the eighth joint and nearly thrice its length : head and chest dull, punctured : abdomen depressed, smooth, shining, bronze-black, much shorter but hardly narrower than the chest; its length scarcely exceeding its breadth : legs piceous : wings colourless; veins tawny. Length of the body $\frac{1}{3}$ line; of the wings $\frac{2}{3}$ line.

Encyrtus Hydramon. Fem. Viridis, abdomine aneo, antennis pedibusque nigris, tarsis fulvis, alis limpidis. Page 135.

Head and chest convex, shagreened, bright green: head a little broader than the chest: eyes and eyelets red: feelers black, clavate, hardly longer than the chest; first joint long, slender, green; second cup-shaped; the following joints from the third to the eighth successively decreasing in length; club spindle-shaped, a little broader than the eighth joint and more than thrice its length : chest short-elliptical: shield of the mid-chest long, conical ; axillæ just meeting on the back; scutcheon large, subrhomboidal : ahdomen obconical, depressed, shagreened, dark bronze colour, greenish towards the base, a little narrower and very much shorter than the chest : legs black; feet dark tawny; middle-feet and four hinder-knees pale yellow: wings colourless; veins piceous. Length of the body $\frac{2}{3}$ line; of the wings $1 \frac{1}{3}$ line.

Encrrtus Philotis. Mas. Niger, antennis tarsisque fulvis, alis limpidis. Page 135.

Body black, rather narrow: head and chest convex, thickly punctured, rather hairy: head as broad as the chest: eyes and eyelets piceous : feelers dull tawny, filiform, rather hairy, nearly as long as the body; first joint long, slender, black; second cupshaped, black; the following joints from the third to the eighth long, linear, of nearly equal length; club spindle-shaped, nearly twice the length of the eighth joint : chest nearly elliptical : shield of the mid-chest long; axillæ parted by a short interval on the back; scutcheon nearly obconical: abdomen obconical, depressed, smooth, a little narrower and very much shorter than the chest; segments of nearly equal size: legs black; knees black; knees and feet dark tawny, middle-feet pale tawny : wings colourless; veins piceous. Length of the body $\frac{3}{4}$ line; of the wings $1 \frac{1}{2}$ line. 
Encyrtus Idmon. Fem. Niger, tarsis fulvis, alis limpidis. Page 135.

Black: head and chest convex, shining, coarsely punctured, clothed with a few scattered hairs: head as broad as the chest: eyes dark red : feelers black, clavate, a little longer than the chest ; first joint long, slender; second cup-shaped ; the following joints from the third to the eighth short, successively and rapidly increasing in breadth; club elliptical, very much broader than the tenth joint and full thrice its length : chest short-elliptical : shield short and broad; axillæ nearly meeting on the back; scutcheon obeonical; abdomen spindle-shaped, depressed, smooth, a little narrower and very much shorter than the chest: legs black; feet dark tawny, with piceous tips: wings colourless; veins piceous. Length of the body $1 \frac{1}{2}$ line; of the wings 2 lines.

Encyrtus Alcmon. Fem. Nigro-viridis, abdomine nigro-ceneo, antennis pedibusque fulvis, alis subfulvis. ENCYRTUs ænescens? Zetterstedt, Ins. Lapp.

Body narrow : head and chest convex, greenish black, with a bronze tinge, almost smooth : eyes and eyelets piceous : feelers subclavate, tawny, a little longer than the chest; first joint short and stout; second also short; third long; fourth and following joints short and successively decreasing in length; club conical: chest elliptical : shield rather flat; axillæ meeting on the back; scutcheon subrhomboidal : hind-chest short : abdomen elliptical, brassy black, flat, shining, a little shorter than the chest: legs piceous; middlefeet pale tawny ; fore-legs and hind-feet dark tawny : wings slightly tawny, rather short and narrow ; veins yellow ; ulna and radius piceous. Length of the body $\frac{1}{2}$ line; of the wings $\frac{3}{4}$ line.

\section{Encyrtus Genetyllis. Fem. Nigro-aneus, capite abdominis- que basi viridibus, antennis pedibusque nigris, tarsis fulvis, alis limpidis. Page 135.}

Body rather narrow: head and chest convex : head dark green, as broad as the chest: eyes dark red: feelers black, thick, hairy, clavate, shorter than the chest; first joint stout, spindle-shaped; second long cup-shaped; the following joints very short, but with a successive increase in breadth and length; club elliptical, very much broarler than the preceding joint, and about tive times its length: chest nearly linear, brassy-black, coarsely punctured; axillæ quite 
meeting on the back : abdomen long-obconical, depressed, smooth, shining, dark bronze, bright green at the base, a little narrower but hardly shorter than the chest : legs black ; knees yellow; feet tawny; middle-shanks and middle-feet luteous: wings colourless, rather narrow; veins tawny; ulna piceous. Length of the body $1 \frac{1}{2}$ line; of the wings 1 line.

Encrrtus Anthores. Fem. Nigro-viridis, antennis fulvis, pedibus nigris, tarsis luteis, alis fuscis. Page 135.

Allied to Epicopterus choreiformis, Eunotus cretaceus, Encyr tus encopiformis and E. ( $\mathbb{E}$ nasius) Hyettus. Body greenish black, very stout and short: head hardly broader than the chest, very coarsely excavated: eyes piceous: feelers tawny, slender, subclavate, a little longer than the chest; first joint long, slender, dull yellow, traversed by a broad piceous band; second piceous; third small ; the following joints successively increasing in breadth ; club spindle-shaped, flat, a little broader than the preceding joint and about twice its length: chest broad, thickly punctured: fore-chest very short, but visible above : shield of the mid-chest very short; its length less than one-half of its breadth ; scutcheon short-obconical; axillæ parted from each other by a short interval : abdomen obconical, depressed, thinly punctured, a little shorter but not narrower than the chest; its length slightly exceeding its breadth : legs deep black : feet luteous; shanks slightly curved: fore-wings brown, hairy, broad, rather short; veins piceous ; radius a little longer than the ulna; cubitus a little longer than the radius, with which it forms a very acute angle: hind-wings colourless, narrow. Length of the body $\frac{1}{2}$ line; of the wings $\frac{3}{4}$ line.

Encyrtus Idya. Fem. Niger, capite nigro-aneo, antennis nigro-piceis fulvo cinctis, tibiis intermediis tarsisque fulvis, alis limpidis. Page 135.

Body short : head and chest convex : head dark copper-colour, finely punctured, as broad as the chest: eyes and eyelets piceous ; the former rather large: feelers clavate, a little longer than the chest; first joint black, long, spindle-shaped; second long cupshaped, piceous; the following joints to the seventh tawny, very short; club spindle-shaped, black, as long as the six preceding joints : chest short-oval, black, coarsely punctured : fore-chest short, visible above; its length about one-fourth of its breadth: shield of the mid-chest short, broad; axillæ nearly meeting on the back; scutcheon nearly obconical: hind-chest extremely short : abdomen 
oval, black, smooth, depressed above, deeply keeled beneath, a little shorter but hardly narrower than the chest: legs black; feet and middle-shanks tawny: wings colourless; veins tawny, piceous towards the tips. Length of the body $\frac{2}{3}$ line; of the wings $1 \frac{1}{4}$ line.

Entedon Isander. Mas. Niger, antennis pedibusque flavis, alis limpidis. Page 140.

Body black, slender, shining : head and chest convex; head a little broader than the chest: eyes and eyelets piceous : feelers filiform, very slender, pale yellow, a little darker towards the tips, not much shorter than the body ; first joint long and slender; second a little thicker ; third and following joints long and linear : chest elliptical : petiole short: abdomen depressed, a little narrower and shorter than the chest: legs pale yellow, long and slender; hindthighs piceous: wings colourless, rather long and narrow; veins pale tawny; ulna longer than the bumerus; radius and cubitus extremely short. Length of the body $\frac{1}{3}$ line; of the wings $\frac{3}{4}$ line.

Entedon Nurscia. Mas. Cyaneo-viridis purpureo varius, abdomine aneo basi viridi, antennis piceis, pedibus luteis, alis limpidis. Page 140.

Head and chest convex, almost smooth: head green, short, impressed in front, a little broader than the chest : eyes and eyelets red: feelers piceous, filiform, a little longer than the chest; first joint tawny, dilated, with a piceous line above; second small; third and following joints long, linear, of nearly equal size : chest bluish green with a purple tinge: sutures of the parapsides very distinct and deeply marked; axillæ parted by one-third of the breadth of the chest; scutcheon subrhomboidal, rather long: hind-chest of moderate size, obconical, declining, ridged: petiole not very long : abdomen nearly oval, dark bronze, smooth, depressed, bright green at the base, narrower and much shorter than the chest; metapodeon occupying about one-third of the back; octoon and following segments of moderate and nearly equal size: legs luteous; hips green : wings colourless or with a very slight brown tinge; veins piceous ; ulna full twice the length of the humerus; radius about one-third of the length of the ulna ; cubitus very short, not half the length of the radius. Length of the body $\frac{3}{4}$ line; of the wings $1 \frac{1}{2}$ line. 
Entedon Sauros. Fem. Viridis, micans, cyaneo ceneoque va* rius, antennis nigris, pedibus flavis, alis limpidis. Page 140 .

Brilliant green: head and chest convex, finely shagreened, with a slight bluish tint: head very short, impressed in front, hardly so broad as the chest: eyes and eyelets red: feelers black, filiform, much shorter than the chest; first joint long, tawny towards the base ; second cup-shaped ; the following joints successively decreasing in length; club pointed, longer than the preceding joint: chest long-elliptical: fore-chest hardly seen above : shield of the mid-chest long ; sutures of the parapsides distinct : axillæ parted by half the breadth of the chest; scutcheon subrhomboidal, with a slight suture on each side; these sutures converge together and form an angle near the tip: hind-chest rather long, obconical, declining, nearly smooth; its fore-shield distinct : petiole very short: abdomen elliptical, smooth, with a brassy tinge, depressed above, hardly keeled beneath, much shorter but hardly narrower than the chest; segments of nearly equal size : legs very pale yellow; hips green: wings colourless; fore-wings broad; veins piceous; ulna rather more than twice the length of the humerus ; radius full half the length of the ulna, reaching very near the tip of the wing; cubitus very short; wing-brand small. Length of the body $\frac{2}{3}$ line; of the wings $1 \frac{1}{3}$ line.

Entedon Axia. Mas. Viridis, capite cyaneo, abdomine aneoviridi, antennis nigris, pedibus flavis, alis limpidis. Page 140.

Head and chest convex, beset with a few scattered bristles: head thick, blue, almost smooth, a little broader than the chest, not impressed in front: eyes and eyelets red: feelers black, filiform, very much shorter than the chest; first joint long, slender, pale tawny, white at the tip ; second long cup-shaped; third and following joints short; club ending in a bristle, about twice the length of the preceding joint: chest green, oval, well developed, very finely shagreened: fore-chest very short: shield of the mid-chest rather roughly shagreened; sutures of the parapsides very distinct; axillæ parted by one-fourth of the breadth of the chest; scutcheon subrhomboidal, rather large : hind-chest large, bluish green, obconical, declining, roughly excavated: petiole long, slender, finely punctured, a little longer than the hind-chest: abdomen æneous green, smooth, depressed, a little louger than broad; metapodeon occupying about one-fourth of the back; octoon and ennaton of moderate 
size; decaton and protelum short; paratelum long; telum very small : legs bright yellow; hips green: wings colourless; forewings broad; veins tawny; ulna about twice the length of the humerus; radius not one-sixth of the length of the ulna; cubitus shorter than the radius: wing-brand very small. Length of the body $\frac{3}{4}$ line; of the wings $1 \frac{1}{2}$ line.

Entedon Antaradus. Mas. Niger, abdomine nigro-aneo basi cyaneo-viridi, antennis pedibusque nigris, tarsis fulvis, alis limpidis. Page 140.

Body short, black: head and chest convex : head very short: eyes and evelets red : feelers black, moniliform, very slender, longer than the chest ; first joint very long and slender ; second and following joints elliptical, slightly petiolated ; club slender, pointed, much longer than the fifth joint: chest smooth, very short, hardly broader than long: fore-chest not visible above: petiole very short: abdomen bronze-black, depressed, smooth, nearly conical, bluish green at the base, a little longer and narrower than the chest: legs black, slender; feet tawny: wings colourless; forewings very broad; veins tawny; ulna much longer than the humerus; radius and cubitus extremely short; wing-brand very small. Length of the body $\frac{1}{3}$ line; of the wings $\frac{3}{4}$ line.

Entedon Erxias. Mas. Niger, abdomine basi cyaneo-viridi, antennis pedibusque piceis, tarsis flavis, alis limpidis. Page 140.

Body short: head and chest black, smooth, convex : head very short, impressed in front, as broad as the chest : eyes and eyelets dark red: feelers piceous, setaceous, a little longer than the chest ; first joint long, stout, tawny: chest elliptical : petiole very short : abdomen short-elliptical, black, depressed, shorter but hardly narrower than the chest, bluish green at the base: legs piceous; feet and tips of the shanks yellow: wings colourless; veins pale tawny; ulna much longer than the humerus; radius and cubitus extremely short. Length of the body $\frac{1}{4}$ line; of the wings $\frac{1}{2}$ line.

Entedon Nænia. Mas. Eneus, abdomine viridi.cenev, antennis nigris, pedibus flavis, alis subfulvis. Page 140.

Body brass colour : head and chest convex, finely shagreened : head very short, impressed in front, hardly broader than the chest: 
eyes and eyelets red: feelers black, slender, hairy, filiform, a little shorter than the chest; first joint long and slender, pale tawny towards the base ; second cup-shaped ; the following joints linear, rather long; club pointed, nearly twice the length of the last joint: chest oval : fore-chest extremely short: shield of the mid-chest less finely shagreened than are the other segments; sutures of the parapsides very distinct; axillæ parted by less than one-third of the breadth of the chest: scutcheon subrhomboidal, rather long: hindchest obconical, declining, rather large, nearly smooth, with a ridge along the middle and a rim on each side : petiole very short : abdomen depressed, smooth, greenish brass colour, increasing in breadth from the base till near the tip, rather shorter and narrower than the chest; metapodeon occupying less than one-third of the back; octoon and following segments of nearly equal size: legs yellow; hips brassy ; tips of the feet brown: wings slightly tawny; veins tawny; ulna rather more than twice the length of the humerus ; radius not one-fourth of the length of the ulna; cubitus very short, not half the length of the radius : wing-brand extremely small. Length of the body 1 line; of the wings 2 lines.

Entedon Stennos. Mas. Eneo-viridis, cupreo- purpureoque varius, antennis nigris, pedibus luteis, femoribus piceis, alis limpidis. Page 140.

Head and chest convex, shagreened, bronze-green, varied with copper-colour and purple; the latter colours predominate towards the head and the fore-part of the chest: head thick, not impressed in front, a little broader and more finely shagreened than the chest; crown broad: eyes and eyelets dark red : feelers setaceous, black, slender, much shorter than the chest; first joint long, rather stout; second cup-shaped; the following joints slightly petiolated; club pointed, very much longer than the preceding joint: chest longoval, well developed: fore-chest very short; its length not more than one-tenth of its breadth: sutures of the parapsides distinct, especially towards the hind border of the shield; axillæ parted by nearly one-half of the breadth of the chest; scutcheon subrhomboidal : hind-chest long-obconical, declining, with a ridge along the back and a rim on each side : petiole shagreened, stout, nearly onethird of the length of the abdomen, greenish brass colour, depressed, smooth, nearly conical, hardly more than half the length of the chest; metapodeon long; the following segments of moderate length: legs luteous; hips brass colour; thighs piceous; foreshanks, fore-feet and tips of four hinder-feet tawny: wings colourless ; veins piceous ; ulna nearly twice the length of the humerus; 
radius less than one-third of the length of the ulna; cubitus not half the length of the radius; wing-brand extremely small. Length of the body $\frac{3}{4}$ line; of the wings $1 \frac{1}{2}$ line.

Entedon Amadocus. Fem. Cyaneo-viridis, scutello purpureoaneo, antennis nigris, tibiis tarsisque flavis, alis subfuscis. Page 140.

Head and chest convex, dark bluish green : head very finely shagreened, slightly impressed in front, hardly broader than the chest : eyes and eyelets dark red : feelers black, slightly setaceous, not longer than the chest, rather hairy; first joint long; second very small ; the following joints successively decreasing in length; club conical, pointed, much longer than the preceding joint: chest long-oval: fore-chest not visible above: shield of the mid-chest rather long; sutures of the parapsides distinct towards the hind border of the shield ; axillæ parted by nearly one-half of the breadth of the shield; scutcheon purplish bronze colour, nearly obconical: hind-chest well deveioped, obconical, declining; its fore-shield as long as the following segment: petiole very short: abdomen bluish green, smooth, rather hairy, depressed above, hardly keeled beneath, much shorter and a little narrower than the chest ; metapodeon occupying nearly two-thirds of the back: octoon and ennaton of moderate size; the following segments very short: legs yellow; hips and thighs bluish green; fore-feet and tips of four hinder-feet tawny : wings slightly brown; veins piceous; ulna rather more than twice the length of the humerus; radius full one-third of the length of the ulna; cubitus much less than half the length of the radius, but longer than is usual in this genus; wing-brand of moderate size. Length of the body $\frac{3}{4}$ line; of the wings $1 \frac{3}{4}$ line.

Entedon Temena. Mas. Viridis, capite aureo-viridi, abdomine cyaneo-aneo basi viridi, antennis nigris, pedibus albis, alis limpidis. Page 140.

Head and chest convex : head bright golden green, smooth, not impressed in front, bluish green behind, a very little broader than the chest: eyes and eyelets red: feelers black, filiform, not more than half the length of the chest; first joint long, rather slender; second long cup-shaped; the following joints linear, rather long; club pointed, much longer than the preceding joint: chest green, long-oval, well developed : fore-chest very short, but distinct: sutures of the parapsides deeply marked; axillæ parted by rather more than one-third of the breadth of the chest; scutcheon sub- 
rhomboidal, rather long and large : hind-chest long, obconical, declining, with a ridge down the middle and a rim on each side; fore-shield very short: petiole slender, cylindrical, shagreened, almost as long as the hind-chest: abdomen bluish bronze, depressed, smooth, a little longer than broad, narrower than the chest and hardly half its length; metapodeon green, occupying full one-third of the back; octoon and following segments very short: legs white ; hips green; tips of the feet brown: wings colourless; veins dark tawny ; ulna about twice the length of the humerus; radius onefourth of the length of the ulna; cubitus not half the length of the radius; wing-brand very small. Length of the body $\frac{3}{4}$ line; of the wings $1 \frac{1}{2}$ line.

Entedon Metagenes. Mas. Purpureo-cyaneus, capite scutelloque viribibus, abdomine nigro basi viridi, antennis pedibusque nigris, tarsis fulvis, alis limpidis. Page 140.

Body smooth, shining: head and chest convex : head green, very short, impressed in front, as broad as the chest: eyes and eyelets dark red : feelers black, nearly filiform, hardly longer than the chest ; first joint long, rather slender, slightly curved ; second somewhat dilated ; the following joints long, linear : club pointed at the tip, much longer than the preceding joint: chest elliptical, very dark blue, tinged with purple; scutcheon green : petiole very short: abdomen short-elliptical, depressed, black, green at the base, a little shorter and broader than the chest: legs black; feet tawny; tips piceous: wings colourless; veins piceous; ulna nearly twice the length of the humerus; radius and cubitus extremely short. Length of the body $\frac{1}{2}$ line; of the wings 1 line.

Entedon Ergetelis. Fem. Cyaneo-viridis, capite aureo-viridi, abdomine aneo, antennis nigris, pedibus albis, alis limpidis. Page 140.

Head and chest convex : head golden green, very finely shagreened, slightly impressed in front, hardly as broad as the chest : eyes red, rather large : feelers black, filiform, much shorter than the chest; first joint pale tawny; second rather short; the following joints long, but successively decreasing in length; club pointed, longer than the preceding joint : chest elliptical, bluish green, rather coarsely shagreened : fore-chest very short, just visible above: sutures of the parapsides rather distinct; axillæ parted by half the breadth of the chest; scutcheon subrhomboidal; sutures inclined towards each other near the tip, but not meeting : hind-chest short : 
petiole very short: abdomen smooth, depressed, bronze colour, hardly keeled beneath, shorter but scarcely narrower than the chest: segments of nearly equal size : leg's white; hips green : wings colourless; veins piceous; ulna full twice the length of the humerus ; radius about one-third of the length of the ulna ; cubitus one-fourth of the length of the radius; wing-brand small. Length of the body $\frac{1}{2}$ line: of the wings 1 line.

Entedon Adreus. Mas. Cyaneus, abdomine viridi, antennis nigris, pedibus flavis, femoribus viridibus, alis limpidis. Page 140.

Head and chest convex, blue, finely shagreened : head broader than the chest, short, impressed in front: eyes and eyelets dark red : feelers black, nearly moniliform, hardly longer than the chest ; first joint long, dilated; second short; third and following joints longer; club long, pointed, much longer than the preceding joint: chest elliptical: sutures of the parapsides distinct: petiole rather long : abdomen dark green, convex, smooth, shorter and narrower than the chest: legs pale yellow; hips and thighs green; tips of feet brown; fore-shanks and fore-feet tawny: wings colourless ; veins tawny; ulna nearly twice the length of the humerus; radius hardly one-half the length of the ulna ; cubitus short, but longer than usual in the genus. Length of the body $\frac{1}{2}$ line : of the wings 1 line.

Entedon Icetas. Mas. Aneo-viridis, abdomine aneo, antennis nigris, pedibus flavis, femoribus fulvis, alis subfulvis. Page 141.

Head and chest convex: head bright green, very short, impressed in front, very finely shagreened, a little broader than the chest: eyes and eyelets red : feelers black, setaceous, shorter than the chest; first joint long, rather stout; second long cup-shaped; the following joints slightly petiolated; club pointed, ending in a bristle, much longer than the preceding joint: chest elliptical, brassy green, finely shagreened : fore-chest extremely short : shield of the mid-chest short, roughly shagreened; sutures of the parapsides very distinct; axillæ parted by one-third of the breadth of the chest ; scutcheon subrhomboidal, long : hind-chest long, obconical, declining: petiole very short : abdomen copper-colour, smooth, depressed, slightly increasing in breadth from the base till near the tip, shurter but hardly narrower than the chest; metapodeon rather long; octoon and following segments of moderate size: legs pale 
yellow; hips brassy; thighs tawny: wings slightly tawny; veins tawny; ulna about twice the length of the humerus; radius not near one-fourth of the length of the ulna ; cubitus very short ; wingbrand very small. Length of the body $\frac{3}{4}$ line; of the wings $1 \frac{1}{2}$ line.

Eulophus Mamurits. Mas. Viridis, abdomine nigro-æneo basi viridi, antennis nigris, tarsis piceis basi flavis, alis subgriseis. Page 144.

Head and chest green: head a little broader than the chest : eyes and eyelets red : feelers black, hairy, 3-branched, subclavate, a little longer than the chest; first joint green, spindle-shaped : chest long-elliptical, very finely shagreened: shield of the mid-chest rather flat; sutures of the parapsides indistinct; axillæ parted by full one-third of the breadth of the chest : hind-chest nearly smouth, obconical, declining ; its fore-shield of moderate length: abdomen linear, depressed, bronze-black, green at the base, narrower but not shorter than the chest: legs green; feet piceous; knees and base of four hinder-feet yellow : wings slightly gray; veins tawny; ulna a little shorter than the humerus; radius less than one-half of the length of the ulna; cubitus much shorter than the radius; wingbrand very small. Length of the body $\frac{2}{3}$ line; of the wings 1 line.

Eulophus Rhamnius. Mas. Viridis, capite cyaneo, scutello nigro, abdomine nigro-ceneo, basi fulvo maculato, antennis pedibusque nigris, tibiis anticis tarsisque piceis, alis subgriseis. Page 144.

Head blue, as broad as the chest: eyes and eyelets piceous: feelers black, hairy, subclavate, a little longer than the chest; first joint spindle-shaped : chest long-elliptical, dark green, shagreened : fore-chest very short: shield of the mid-chest broad; sutures of the parapsides indistinct; axillæ parted by one-third of the breadth of the chest; scutcheon subrhomboidal, black: hind-chest rather large, obconical, declining, finely shagreened, with a ridge along the back: petiole very short: abdomen shorter and much narrower than the chest, very slightly increasing in breadth from the base to near the tip, depressed, smooth, bronze-black, with a dark tawny spot near the base which is green: legs black; knees, feet, and fore-shanks piceous : wings slightly gray; ulna full as long as the humerus; radius more than half the length of the ulna; cubitus less than one-half the length of the radius; wing-brand small. Length of the body $\frac{3}{4}$ line; of the wings $1 \frac{1}{2}$ line. 
Eulophus stygius. Fem. Niger, pedibus fulvis, femoribus nigris, alis fuscis. Page 144.

Black, hairy, very finely shagreened, almost smooth: head consex: eyes and eyelets red: feelers black, clavate, hairy, shorter than the chest; first joint long, slender; second cup-shaped; the following joints successively decreasing in length; club conical, a little longer than the preceding joint; abdomen long-elliptical, smooth, depressed, hairy, much shorter and a little broader than the chest; metapodeon occupying nearly one-fourth of the back; the following segments of moderate size: legs tawny; hips and thighs black; tips of the latter tawny: wings brown; veins piceous; ulna much longer than the humerus; radius more than half the length of the ulna ; cubitus not half the length of the radius ; wingbrand very small. Length of the body $1 \frac{1}{4}$ line; of the wings $2 \frac{1}{3}$ lines.

Eulophus Pythodorus. Fem. Eneus, capite viridi, abdomine purpureo basi viridi, antennis femoribusque nigris, tibiis piceis, tarsis flavis, alis limpidis. Page 144.

Body short and broad : head and chest convex, very finely shagreened: head short, dark green, much impressed in front: eyes and eyelets dark red : feelers black, clavate, shorter than the chest; first joint long, slender ; second cup-shaped ; three following joints successively decreasing in length; club long, pointed, full twice the length of the preceding joint: chest bronze colour, short-elliptical: fore-chest very short: shield of the mid-chest broad; sutures of the parapsides indistinct; axillæ parted by nearly half the breadth of the chest; scutcheon subrhomboidal : hind-chest short, transverse, declining, with a ridge along the middle : petiole very short: abdomen short-elliptical, dark purple, very finely shagreened, green at the tip, bright green at the base, depressed above, hardly keeled beneath, a little shorter and broader than the chest; metapodeon occupying full one-sixth of the back; the following segments of moderate and nearly equal size: hips and thighs black; shanks piceous; feet yellow with piceous tips; fore-shanks and fore-feet tawny: wings colourless; humerus longer than the ulna; radius less than half the length of the ulna; cubitus shorter than the radius; wing-brand small. Length of the body 1 line; of the wings 2 lines. 
Eulophus Acalle. Mas. Cyaneus, abdomine purpureo-aneo flavo-maculato basi viridi, antennis nigris, pedibus albis, femoribus nigris, tibiis apice piceis, alis limpidis. Page 144.

Head and chest blue : feelers black, hairy, a little shorter than the chest: shield of the mid-chest flat; scutcheon black: hindchest nearly smooth, obconical, declining; its fore-shield of moderate length: abdomen nearly linear, depressed, smooth, purplish bronze, yellow towards the base which is green: legs white; hips and thighs black; tips of the shanks piceous ; fore-shanks and forefeet tawny: tips of the four hinder-feet brown : wings colourless; fore-wings broad; veins tawny; ulna shorter than the humerus; radius much shorter than the ulna; cubitus about one-third of the length of the radius; wing-brand small. Length of the body 1 line; of the wings $1 \frac{3}{4}$ line.

Eulophus Veturius. Mas. Viridis, abdomine purpureo-aneo basi viridi, antennis tibiisque piceis, tarsis fulvis basi flavis, alis limpidis. Page 144.

Green : head broader than the chest: eyes and eyelets dark red: feelers piceous, 3-branched, a little longer than the chest; first joint green, spindle-shaped: chest oval, finely shagreened : shield of the mid-chest broad, rather short ; sutures of the parapsides indistinct; scutcheon subrhomboidal: hind-chest nearly smooth, obconical, declining, its fore-shield of moderate length : abdomen linear, depressed, purplish bronze, smooth, green at the base, hardly shorter but very much narrower than the chest: hips and thighs green ; shanks piceous ; knees and feet tawny; base of four hinderfeet pale yellow : wings colourless ; fore-wings rather broad ; veins tawny; ulna a little longer than the humerus; radius less than half the length of the ulna; cubitus much shorter than the radius; wing-brand very small. Length of the body $\frac{3}{4}$ line; of the wings $1 \frac{1}{2}$ line.

EuLophus Scyllis. Fem. Viridis, abdomine aneo, antennis nigris, pedibus fulvis, alis subfulvis. Page 144.

Head and chest green, convex, finely shagreened: head as broad as the chest: eyes and eyelets red: feelers black, clavate, shorter than the chest ; first joint long, slender ; second cup-shaped ; the four following joints successively decreasing in length; club 
conical, longer than the preceding joint: chest long-elliptical : fore-chest rather long, short-conical, its length full one-half of its breadth: sutures of the parapsides very distinct; axillæ parted by one-fourth of the breadth of the chest; scutcheon subrhomboidal: hind-chest well developed, obconical, declining, almost smooth, with a ridge along the back; its fore-shield rather long: petiole very short : abdomen bronze colour, slightly tinged with green, smooth, elliptical, depressed above, slightly keeled beneath, about as long and as broad as the chest; metapodeon occupying about one-sixth of the back; the following segments of moderate size: legs tawny ; hips green: wings slightly tawny; veins tawny; ulna as long as the humerus; radius much shorter than the ulna; cubitus not half the length of the radius; wing-brand very small. Length of the body $1 \frac{1}{4}$ line; of the wings $2 \frac{1}{4}$ lines.

Diglyphus Poppœa. Fem. Viridis, cyaneo purpureo aneoque varius, antennis nigris basi albis, pedibus viridibus albo-cinctis, alis limpidis. Page 145.

Head blue, nearly smooth, bronze on the crown, impressed above, prominent in front, not broader than the chest: eyes and eyelets red: feelers black, stout, clavate, shoxter than the chest; first joint long, slender, white towards the base ; second of moderate size; third and fourth broader; club long-conical, twice the length of the preceding joint : chest green, elliptical : fore-chest extremely short: shield broad, nearly flat above; sutures of the parapsides obsolete; axillæ parted by nearly one-half of the breadth of the chest; scutcheon nearly obconical, with a suture along each side : hind-chest well developed, obconical, slightly declining, nearly smooth, with a slight ridge along the back ; fore-shield large, about one-half of the length of the following segment: petiole very short : abdomen smooth, elliptical, purplish bronze, green at the base, blue at the tip, depressed above, slightly keeled beneath, shorter but hardly broader than the chest; metapodeon occupying full one-half of the back; octoon and ennaton rather large; the following segments extremely short : hips and thighs green; shanks piceous with a green tinge; feet and tips of thighs and of shanks white; foreshanks, fore-feet, and tips of four hinder-feet tawny : wings colourless; veins piceous; ulna nearly as long as the humerus; radius much shorter than the ulna; cubitus not half the length of the radius; wing-brand small. Length of the body $\frac{1}{2}$ line; of the wings $1 \frac{1}{4}$ line. 
Diglyphus Aculeo. Fem. Viridis, abdomine fulvo, antennis pedibusque flavo-fulvis, alis subfulvis. Page 145.

Body very slender: head and chest green, rather flat: head broader than the chest: eyes and eyelets red: feelers pale tawny, clavate, a little shorter than the chest; club much broader than the preceding joint and more than twice its length: chest long-elliptical: petiole very short: abdomen elliptical, tawny, smooth, depressed, a little shorter but not narrower than the chest: legs pale tawny: wings narrow, nearly colourless or slightly tawny; veins tawny; ulna a little longer than the humerus; radius and cubitus very short. Length of the body $\frac{1}{3}$ line; of the wings $\frac{2}{3}$ line.

Var. $\beta$. Feelers and legs yellow.

Tetrastichus Triarius. Mas. Niger, abdomine nigro-aneo, antennis fulvis, pedibus piceis, tarsis flavis, alis limpidis. Page 151.

Body rather short: head and chest black, convex, smooth : head short, as broad as the chest: eyes and eyelets bright red: feelers tawny, adorned with long hairs, not longer than the chest; first joint piceous : chest elliptical : fore-chest very short : shield of the mid-chest broad and long, with a suture along the back; sutures of the parapsides distinct; axillæ parted by more than onethird of the breadth of the chest; scutcheon subrhomboidal : hindchest transverse, declining: petiole very short: abdomen bronzeblack, depressed, linear, shorter and narrower than the chest: legs piceous ; four hinder-knees and feet pale yellow; tips of the latter brown; fore-shanks and fore-feet tawny: wings colourless; veins tawny; ulna longer than the humerus; radius next to none; cubitus about one-fourth of the length of the ulna; wing-brand extremely small. Length of the body $\frac{1}{3}$ line; of the wings $\frac{3}{4}$ line.

Tetrastichus Abydenus. Mas. Niger, abdomine fulvo maculato, antennis fulvis, pedibus flavis, alis limpidis. Page 151.

Head and chest black, convex, shining: head smooth, very short, hardly as broad as the chest: eyes and eyelets red; feelers filiform, tawny, slender, a little longer than the chest, adorned with long hairs ; first joint piceous ; second yellow : chest nearly oval, very finely punctured : fore-chest extremely short: shield of the mid-chest very long, with a slight suture along the middle ; sutures of the parapsides strongly marked; scutcheon nearly obconical, 
with a suture along each side: hind-chest rather large, obconical, declining, with a ridge along the middle; its fore-shield tolerably long: petiole very short: abdomen black, smooth, shining, depressed, nearly linear, with a large tawny spot near the base, a little longer and much narrower than the chest: legs pale yellow; hips black; tips of feet tawny : wings colourless, ample; veins tawny; ulna a little longer than the humerus; radius next to none; cubitus not one-fourth of the length of the ulna; wing-brand very small. Length of the body 1 line; of the wings $2 \frac{1}{4}$ lines.

Tetrastichus Bermius. Mas. Niger, abdomine aneo, antennis fulvis, pedibus flavis, alis limpidis. Page 151.

Head and chest black, convex, smooth, shining : head as broad as the chest: eyes and eyelets bright red: feelers slender, filiform, pale tawny, adorned with long hairs, nearly as long as the body; first and second joints pale yellow : chest short: shield of the midchest broad; sutures of the parapsides very distinct; scutcheon short, with a suture along each side: abdomen bronze-colour, linear, dep. assed, shorter and narrower than the chest: legs pale yellow: wings colourless, deeply fringed; veins tawny ; ulna nearly as long as the humerus: radius next to none; cubitus as usual; wingbrand very small. Length of the body $\frac{1}{2}$ line; of the wings $1 \frac{1}{4}$ line. 











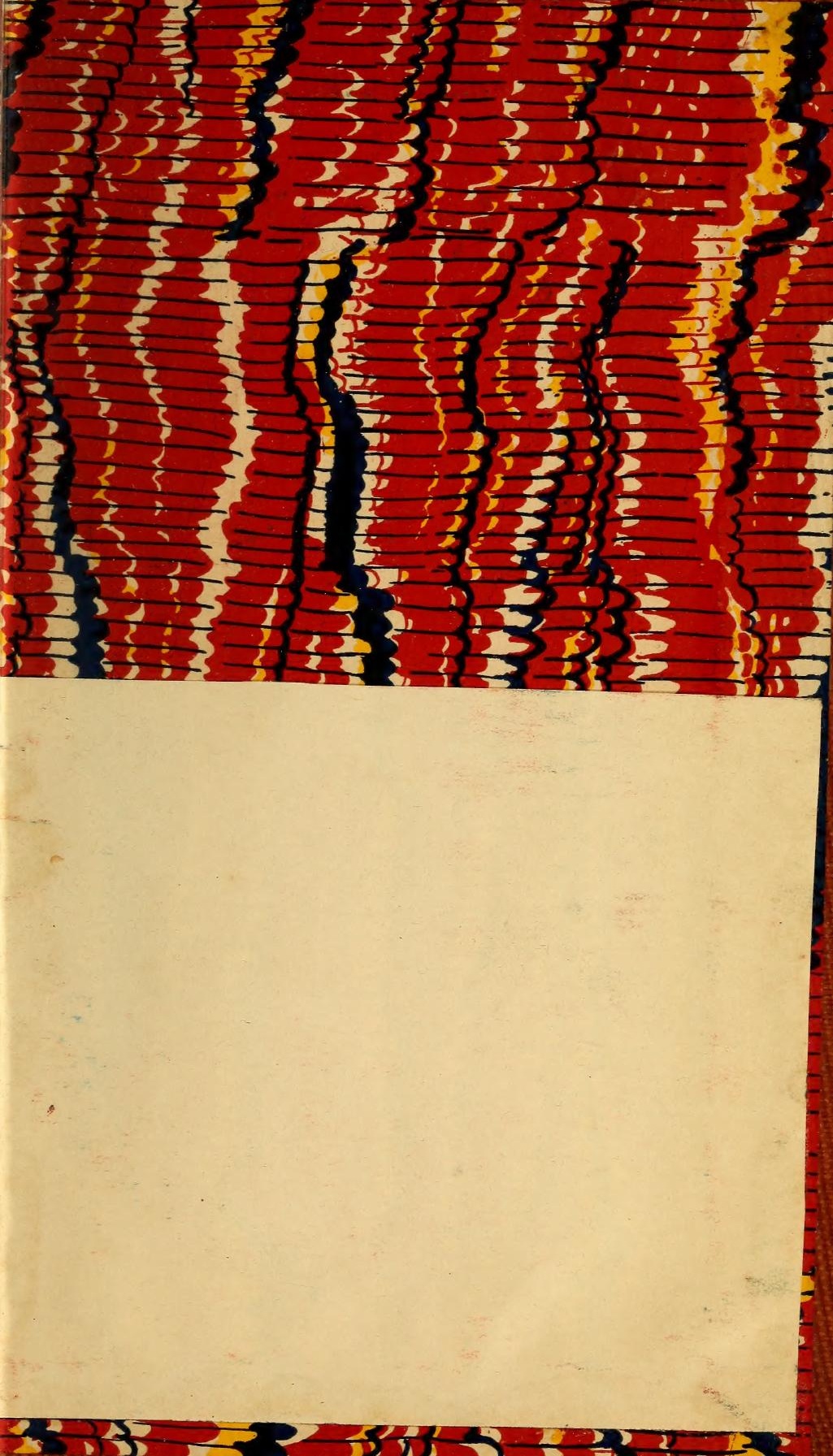


SMITHSONIAN INSTITUTION LIBRARIES 